\title{
Studies of collective effects in pp collisions at the LHC with the balance function for identified particles
}

Zhanna Khabanova 
Copyright (0) 2020 Zhanna Khabanova

Cover designed by Simon Tuckett

Printed by Ipskamp Printing

ISBN: 9789464211092

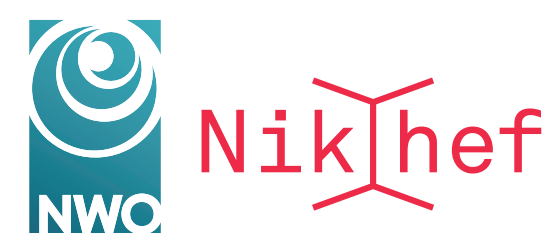

This work is part of the research programme of the Foundation for Fundamental Research on Matter (FOM), which is part of the Dutch Research Council (NWO). 


\section{Studies of collective effects in pp collisions at the LHC with the balance function for identified particles}

Studies van collectieve effecten bij pp botsingen bij de LHC met de balansfunctie voor geïdentificeerde deeltjes (met een samenvatting in het Nederlands)

\section{Proefschrift}

ter verkrijging van de graad van doctor aan de Universiteit Utrecht op gezag van de rector magnificus, prof.dr. H.R.B.M. Kummeling, ingevolge het besluit van het college voor promoties in het openbaar te verdedigen op

woensdag 2 december 2020 des middags te 2.30 uur

door

\section{Zhanna Khabanova}

geboren op 4 april 1992

te Kiev, Oekraïne 


\section{Promotor:}

Prof. dr. R.J.M. Snellings

Copromotor:

Dr. P. Christakoglou 
"I have not failed. I've just found 10,000 ways that won't work."

—Thomas Edison 



\section{Abstract}

A new state of matter, where quarks and gluons are not confined inside hadrons, called quark-gluon plasma (QGP), was predicted to exist at sufficiently high temperatures and energy densities by the theory of strong interactions, quantum chromodynamics (QCD) around 50 years ago. At present, this state can be created in the collisions between heavy ions accelerated to ultra-relativistic speeds, which provides a unique possibility to explore the behaviour of strongly interacting matter under extreme conditions. Experimental measurements at RHIC and the LHC allowed to conclude that the medium formed in heavy-ion collisions is strongly coupled and is well described by relativistic viscous hydrodynamic equations for a perfect liquid. These properties lead to the collective expansion of all particles produced in the collision with a common velocity, i.e. collective flow. For some of these measurements, proton-nucleus and proton-proton collisions, referred to as small systems, where QGP was not expected to form, have been used as a reference. Recently, this expectation has been confronted by intriguing results reported by experiments at RHIC and the LHC that indicate the presence of collective flow also in small systems. The origin of this effect is not fully understood. In particular, it is not clear whether it is caused by the QGP formation or other alternative mechanisms that can lead to similar patterns as hydrodynamic flow without involving the QGP expansion, such as color reconnection. To investigate it better, the balance function which studies the charge-dependent part of two-particle correlations in the relative pseudorapidity $(\Delta \eta)$ and azimuthal angle $(\Delta \varphi)$ of the particle pair can be used. In heavy-ion collisions, this measurement has been used as an effective tool to investigate the properties of the QGP evolution such as the hadronization time, the freeze-out conditions and to characterise its collective motion.

In this thesis, the analysis of the balance function for identified particles in proton-proton collisions at $\sqrt{\mathrm{s}}=5.02 \mathrm{TeV}$ collected by the ALICE experiment is presented. The results are shown for pion, kaon and proton pairs in the low transverse momentum range. In addition, a comparison of the data in pp and $\mathrm{p}-\mathrm{Pb}$ collisions at the same energy is presented. Furthermore, the data in 
pp are compared with predictions from two different tunes of the PYTHIA8 Monte Carlo model: with and without color reconnection. This mechanism leads to correlations between particles at the microscopic level due to the string recombination. New findings presented in this work show that balance function results for identified hadrons are not in agreement with the expected effect of collective flow in small systems. Moreover, neither of PYTHIA8 tunes is able to reproduce experimental data. 


\section{Contents}

Abstract $\quad$ v

1 Introduction $\quad 1$

1.1 The Quark Gluon Plasma . . . . . . . . . . . . . . . . . . . 1

1.2 Heavy-ion collisions . . . . . . . . . . . . . . . 3

1.2.1 Space-time collision evolution . . . . . . . . . . . . 3

1.2 .2 Collision centrality . . . . . . . . . . . . 5

1.2.3 Collectivity in heavy-ion collisions . . . . . . . . . . . 6

1.3 Signs of collectivity in small systems . . . . . . . . . . . 14

2 The balance function $\quad 21$

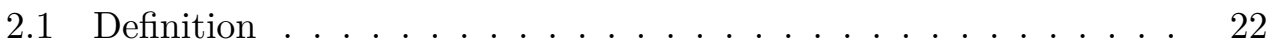

2.2 Balance function width and yield . . . . . . . . . . . . 24

2.2.1 Heavy-ion collisions . . . . . . . . . . . . . . 24

2.2.2 Proton-nucleus and proton-proton collisions . . . . . . . . 27

2.3 Lessons learned from models . . . . . . . . . . . . . . 28

2.4 Where we stand experimentally . . . . . . . . . . . . . 34

3 ALICE Experiment 43

3.1 The Large Hadron Collider . . . . . . . . . . . . . . . . . . . . 43

3.2 ALICE experiment . . . . . . . . . . . . . . . . 44

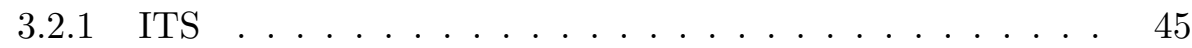

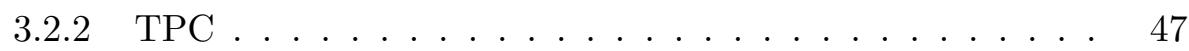

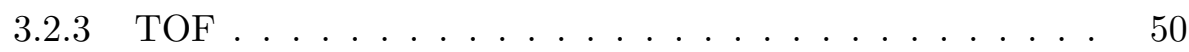

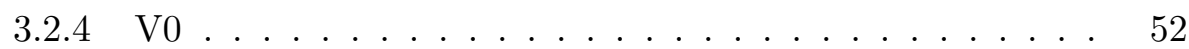

4 Analysis Details $\quad 55$

4.1 Data samples, event and track selection . . . . . . . . . 55

4.1 .1 Data samples . . . . . . . . . . . . . . 55

4.1 .2 Event selection . . . . . . . . . . . . . . . . . 56

4.1 .3 Track selection . . . . . . . . . . . . . . . . . 57

4.2 PID strategy . . . . . . . . . . . . . . . . . . 61

4.3 Analysis method . . . . . . . . . . . . . . . . 65

4.4 Monte Carlo closure test . . . . . . . . . . . . . . . 70

$\begin{array}{llc}5 & \text { Results } & 73\end{array}$ 
5.1 Balance function of charged particles in pp collisions at $\sqrt{\mathrm{s}}=$

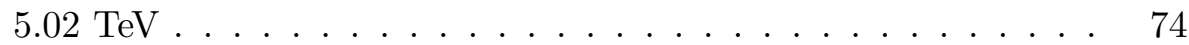

5.1.1 Two-dimensional balance function distributions . . . . . . 74

5.1 .2 Systematic uncertainties . . . . . . . . . . . . 75

5.1 .2 .1 Procedure . . . . . . . . . . . . 76

$5.1 .2 .2 \quad$ Sources . . . . . . . . . . . . 78

5.1 .2 .3 Final values . . . . . . . . . . . . . . . 90

5.1 .3 Balance function projections . . . . . . . . . . 93

5.1 .4 Balance function width and yield . . . . . . . . . 95

5.2 Balance function of pion, kaon and proton pairs in pp collisions at $\sqrt{\mathrm{s}}=5.02 \mathrm{TeV} \ldots \ldots \ldots \ldots \ldots \ldots \ldots$

5.2.1 Two-dimensional balance function distributions . . . . . . 100

5.2.2 Studies of the resonance contribution in the balance func-

tion of pions and kaons . . . . . . . . . . . 103

5.2.3 Balance function projections of pions and protons . . . 107

5.2.4 Balance function width and yield of pions and protons . . 112

5.2.5 Model comparison . . . . . . . . . . . . . . 115

5.3 Comparison of the balance function of charged and identified particles in pp and $\mathrm{p}-\mathrm{Pb}$ collisions at $\sqrt{\mathrm{s}}=5.02 \mathrm{TeV} \ldots \ldots . .123$

$\begin{array}{lr}\text { Summary } & 125\end{array}$

$\begin{array}{lr}\text { Samenvatting } & 129\end{array}$

$\begin{array}{ll}\text { Bibliography } & 133\end{array}$

$\begin{array}{ll}\text { Curriculum Vitae } & 147\end{array}$

$\begin{array}{lr}\text { Acknowledgements } & 149\end{array}$ 


\section{Chapter 1}

\section{Introduction}

\subsection{The Quark Gluon Plasma}

The presence of quarks and gluons inside nucleons was confirmed around 40 years ago by many experimental observations, in processes that involve scattering of high-energy electrons off protons [1] and the measurement of two or three-jet events coming from collisions between electrons and positrons [2], [3]. However, until now no quarks have been observed isolated in nature. This experimental fact motivates the concept of quark confinement, i.e. the postulation that quarks are permanently bound inside composite particles called hadrons. At the same time, in the beginning of 1970's, it was suggested that at high enough temperatures and densities hadrons should lose their individuality, leading to a transition from the nuclear matter to a new state where quarks and gluons are no longer confined inside hadrons. This way, the existence of a new deconfined state of matter, called quark-gluon plasma (QGP), was proposed [4]. In an attempt to provide an intuitive picture for the creation of such a state of matter, various phenomenological descriptions were invented, one of them incorporated in the MIT bag model [5], [6].

In this model, hadrons are represented as finite three-dimensional objects ("bags") containing massless, freely moving quarks and gluons inside. To achieve the confinement, hadrons are embedded in a vacuum that exerts the external inward pressure at the boundary. As long as this pressure is larger than the pressure inside the bag, quarks and gluons remain confined. However, compressing and heating up such hadronic matter beyond a critical temperature, estimated to be around $170 \mathrm{MeV}$ [7], will lead to the bags overlapping with each other and, thus, quarks not belonging to a particular bag anymore. As a consequence, hadronic boundaries will vanish and quarks and gluons will be released. The bag model, 
therefore, predicts in a simplified manner the transition from the hadronic to the deconfined state of matter, QGP.

The existence of such transition is also supported by the theory of the strong interactions, quantum chromodynamics (QCD), through more precise first-principle lattice QCD calculations based on numerical Monte Carlo simulations. In lattice QCD, applied in the regime where the strong coupling constant reaches large values, a non-perturbative approach is required and implemented for solving quantum chromodynamics equations [8]. Quarks and gluons are placed on a discrete lattice of space-time coordinates and their interactions are simulated in thermal equilibrium. These calculations provide the information on the system's thermodynamic parameters that are needed to understand the nature of the transition and the equation of state of the QCD matter in this regime.

Figure 1.1 presents the result of one recent calculation that shows the dependence of the energy density $(\epsilon)$, pressure (p) and entropy (s), normalised by different powers of temperature $(\mathrm{T})$, on the temperature for a system of $2+1$ quark flavours $(\mathrm{u}, \mathrm{d}$ and $\mathrm{s})$, where $2+1$ means that the masses of two light quarks, i.e. $\mathrm{u}$ and $\mathrm{d}$, are approaching 0 , while the strange quark mass is tuned to its physical value [9]. An expected abrupt increase in $\epsilon / \mathrm{T}^{4}$ and $3 \mathrm{p} / \mathrm{T}^{4}$ values is observed which is interpreted as a sudden change in the number of degrees of freedom. This in turns reflects the transition from a medium where hadrons are the relevant degrees of freedom to a state of matter where their constituents are not confined anymore within their hadronic "bags". This increase occurs within the vertical yellow band marking the temperature $\mathrm{T}_{\mathrm{C}}$ range estimated to be $154 \pm 9 \mathrm{MeV}$. The corresponding predicted energy density range is $0.18-0.5 \mathrm{GeV} / \mathrm{fm}^{3}$. All calculations performed so far for QCD with two light quarks with or without the inclusion of a heavier strange quark find a smooth but rapid crossover from the low to high temperature regime [10]. 


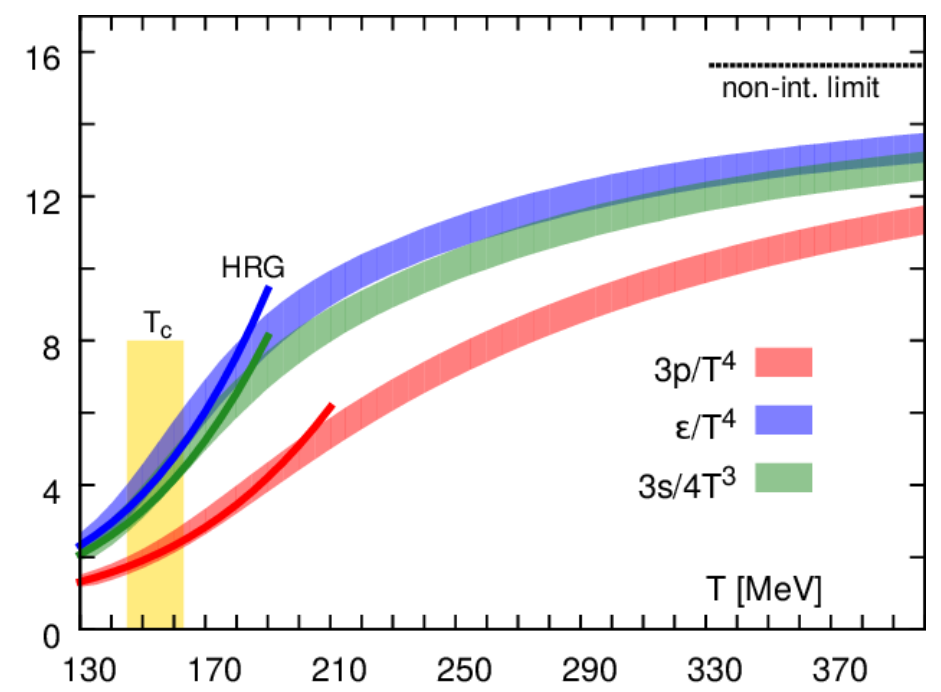

FIGURE 1.1: Lattice QCD prediction for the normalized pressure, energy density and entropy density as a function of the temperature

According to cosmology, similar, extreme conditions prevailed at the first 10 $\mu$ s after the Big Bang [11] making the study of the QGP relevant also on this branch of research. Moreover, it is suggested that the QGP might exist in the core of neutron stars [12]. Nowadays, the conditions in terms of temperature and energy density for the QCD phase transition to take place can be achieved in the laboratory by colliding relativistic heavy ions at The Relativistic Heavy Ion Collider (RHIC) and The Large Hadron Collider (LHC). This opens up a unique possibility for studying the behaviour of strongly interacting matter under extreme conditions as well as recreating and investigating the evolution of the early Universe.

\section{$1.2 \quad$ Heavy-ion collisions}

\subsubsection{Space-time collision evolution}

Before a heavy ion collision takes place, two Lorentz contracted ions are accelerated close to the speed of light. These ions collide and the initial state is characterised by the energy profile which reflects the positions of nucleons participating in the collision that fluctuate from one event to the other. It was recently realised that an accurate description of these initial state fluctuations in models plays a crucial role in reproducing experimental data since they significantly affect the shape of the overlapping zone between colliding nuclei and, therefore, the subsequent determination of the QGP properties discussed later in this section [13]. After the collision, the created system undergoes several stages 
depicted in a schematic view in Figure 1.2 [14]. The vertical axis represents the time direction, while the horizontal axis represents the spatial z direction. The collision of two Lorentz contracted nuclei happens at $(\mathrm{t}, \mathrm{z})=(0,0)$ and leads to a pre-equilibrium phase, characterised by large energy density, where part of hard parton scattering processes start to occur. These processes lead to the production of highly energetic partons that further fragment and give rise to the formation of showers of collimated particles, i.e. jets. Interactions between partons lead to the establishment of local thermal equilibrium. Once equilibrium is achieved around $\tau \sim 1 \mathrm{fm} / \mathrm{c}[15]$, the QGP is created, which then quickly expands and cools down with time. The expansion of the QGP is governed by an equation of state that connects thermodynamic variables such as the energy density, temperature and pressure and is well described by relativistic hydrodynamic models that rely on solving the energy-momentum conservation equations [16] (see Section 1.2.3 for more details). After a critical temperature described in Section 1.1 is reached, the phase transition to a hadron gas occurs. The chemical composition of hadrons is then fixed and inelastic interactions cease. However, hadrons might still interact elastically until the kinetic freeze-out temperature that defines the end of the evolution.

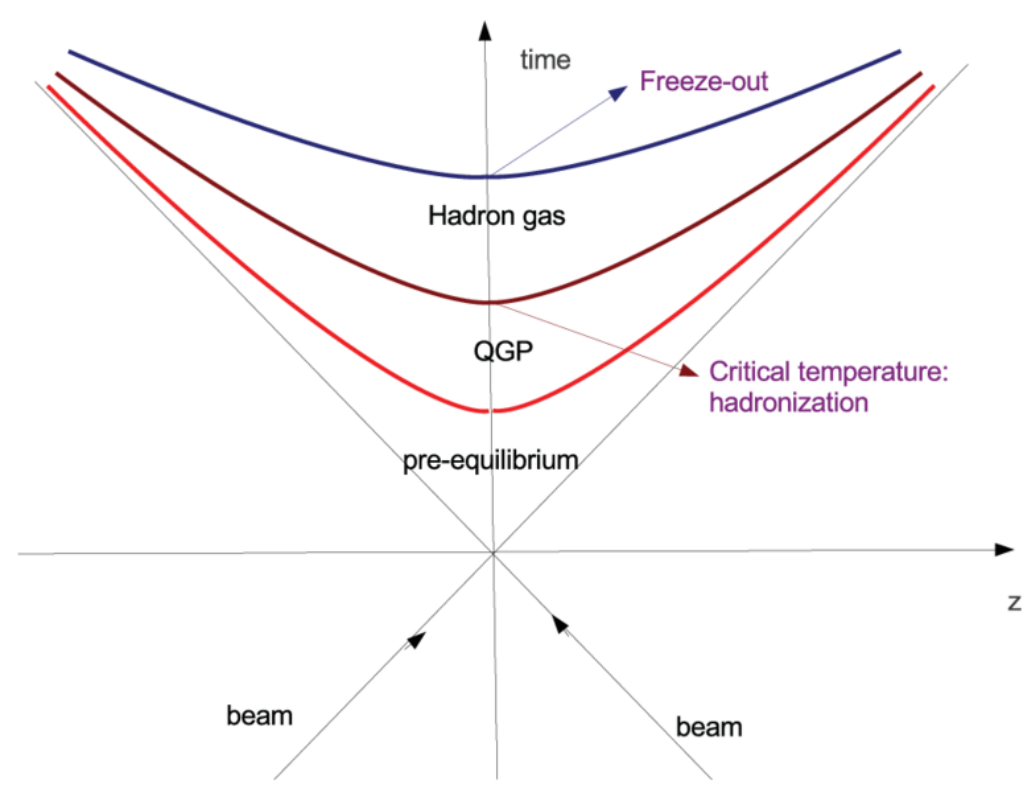

Figure 1.2: A schematic view of the space-time evolution of a heavy-ion collision

Due to a very short QGP lifetime, estimated to be around $10^{-23} \mathrm{~s}$ [17], this phase is not accessible for a direct measurement. However, the formation of a dense and hot medium is reflected in specific characteristics of produced final state particles detected in the experiment. Throughout the previous years, detailed experimental studies have been carried out in order to create and discover the 
QGP and to identify its nature. Among various observables or phenomena that contributed to our understanding of the medium created in heavy-ion collisions, in this thesis few of them are singled out: transverse momentum $\left(\mathrm{p}_{\mathrm{T}}\right)$ spectra of identified particles, angular correlations, (anisotropic) flow and jet quenching. Since many observables are commonly studied as a function of the collision centrality, it is introduced and explained in the following section.

\subsubsection{Collision centrality}

Most of experimental measurements significantly depend on the initial size and the geometry of the overlapping zone between the colliding nuclei. The latter depends on the impact parameter $b$ of a collision defined as the distance between the centers of the two colliding nuclei in a plane perpendicular to the beam axis z, i.e. so-called transverse xy-plane. Central collisions are characterised by a small impact parameter, and hence an overlapping region which is almost round and contains a large number of participating nucleons. On the contrary, peripheral collisions correspond to a large impact parameter value which leads to an elliptical geometry in the overlap region and smaller number of participants. A schematic illustration of three collision types, i.e. peripheral, semi-central and central is shown in the transverse plane from left to right in Figure 1.3 [18]:

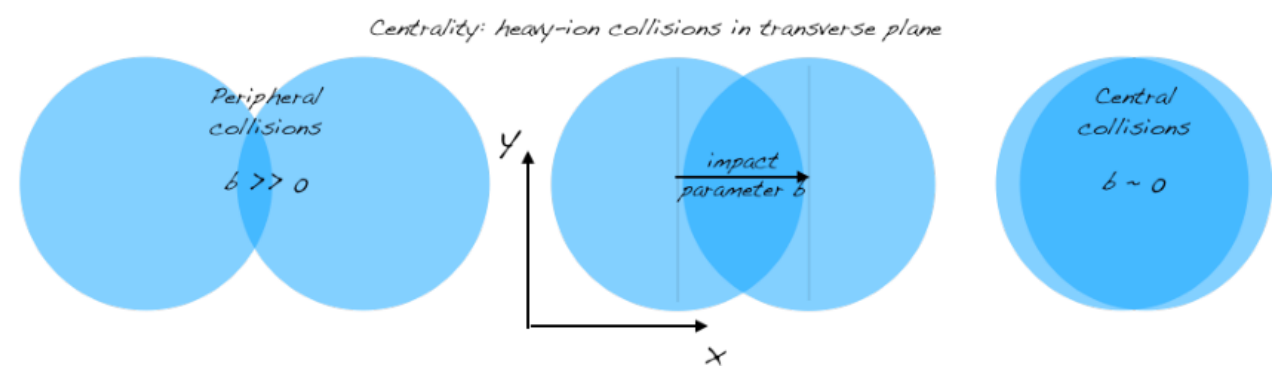

Figure 1.3: A schematic illustration of collision types related to the impact parameter and centrality in transverse plane: peripheral, semi-central and central collisions are shown from left to right

Instead of the impact parameter, the centrality of a collision is estimated by counting the amount of particles produced in a single collision, i.e. the charged particle multiplicity $\mathrm{N}_{\mathrm{ch}}$. The latter is assumed to decrease monotonically with increasing impact parameter. To assign the corresponding mean values of $<\mathrm{b}>$ to a measured centrality, a mapping procedure is performed using the Glauber Monte Carlo model [19]. The idea of this model is to compose two nuclei out of nucleons and simulate their collision process in three dimensions event-byevent. An example of such mapping procedure is shown in Figure 1.4. Centrality classes in the data are defined by counting fractions of the total integral of the 
$\mathrm{dN}_{\text {evt }} / \mathrm{dN}_{\mathrm{ch}}$ distribution represented by dashed lines. After repeating the same procedure with the simulated distribution, where events are classified according to their impact parameter instead of centrality, and comparing data with a simulation, centrality can be derived.

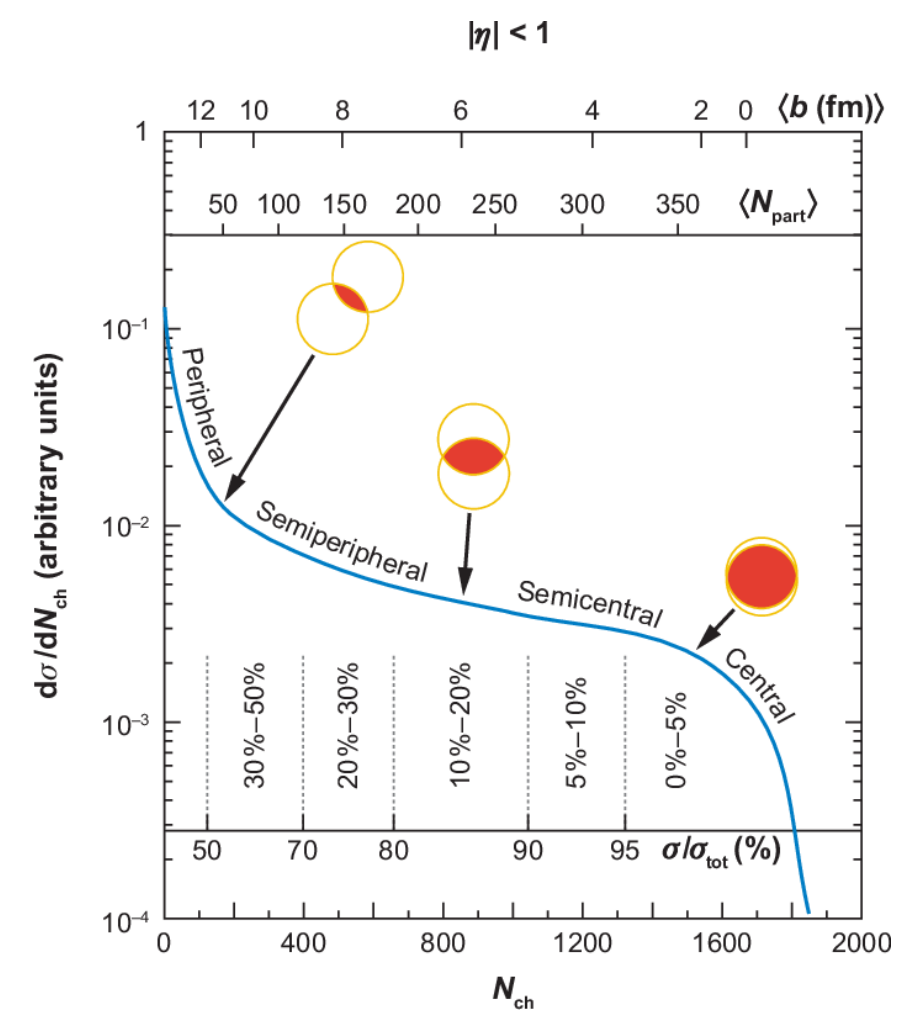

FiguRE 1.4: A cartoon example of the correlation of the charged particle multiplicity $\mathrm{N}_{\mathrm{ch}}$ with Glauber calculated quantity $<\mathrm{b}>$

\subsubsection{Collectivity in heavy-ion collisions}

The initial expectations about the nature of the deconfined state of matter were to detect a gaseous QGP state, i.e. a gas of freely moving (anti-)quarks and gluons in the experiment. However, instead completely different properties were discovered, as discussed in this section.

The expansion of the QGP medium in heavy-ion collisions is successfully described by equations of relativistic viscous hydrodynamics [20], [21]. According to this macroscopic description, the presence of a high thermal pressure in the center of the reaction zone and a vacuum with no pressure surrounding the created fireball causes matter to expand outwards collectively with a common average transverse expansion velocity $\left\langle\beta_{\mathrm{T}}\right\rangle$, called from now on radial flow. The QGP is expected to have a size, a temperature and a lifetime that depend 
on collision energy. The higher the energy of the collision or the size of the created fireball is, the larger the pressure gradient becomes, consequently leading to stronger radial flow in the system. This results in a stronger momentum boost that the produced particles feel on top of the velocity attributed to their thermal motion, $v_{\text {th }}$. Moreover, heavier particles are more sensitive to such a boost due to the direct dependence of transverse momentum $\mathrm{p}_{\mathrm{T}}$ on the mass $\mathrm{m}$ :

$$
p_{T}=\gamma m\left\langle\beta_{T}\right\rangle+v_{t h}
$$

The experimental result demonstrating this dependence is shown in Figure 1.5 [22] that presents the $\mathrm{p}_{\mathrm{T}}$ spectra of pions, kaons and protons for summed charges for the $5 \%$ most central lead-lead $(\mathrm{Pb}-\mathrm{Pb})$ collisions measured by ALICE at $\sqrt{\mathrm{S}_{\mathrm{NN}}}=2.76 \mathrm{TeV}$, compared with results from STAR and PHENIX experiments in gold-gold $(\mathrm{Au}-\mathrm{Au})$ collisions at $\sqrt{\mathrm{s}_{\mathrm{NN}}}=200 \mathrm{GeV}$ [23], [24]. For all particle species, a shift of the particle spectra to higher $\mathrm{p}_{\mathrm{T}}$ values, usually referred to as spectrum hardening, is observed at the higher LHC energy with respect to RHIC. This is due to larger radial flow at the LHC which is about $10 \%$ stronger than at RHIC for central collisions [22]. In addition, a pronounced flattening of the spectra is observed for protons compared with pions and kaons at both energies since heavier particles are more sensitive to an increase in the radial flow.

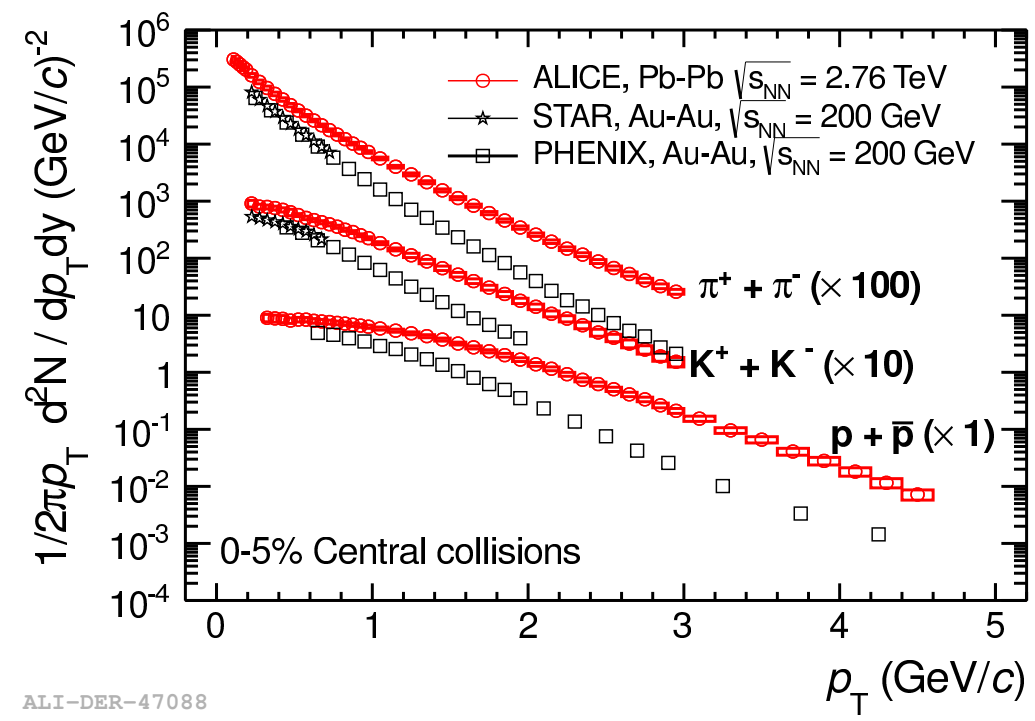

Figure 1.5: Spectra of identified particles $(\pi, \mathrm{K}, \mathrm{p})$ for summed charges in the centrality bin $0-5 \%$ in $\mathrm{Pb}-\mathrm{Pb}$ collisions at $\sqrt{\mathrm{S}_{\mathrm{NN}}}=2.76 \mathrm{TeV}$ from ALICE compared to results from $\mathrm{RHIC}$ in $\mathrm{Au}-\mathrm{Au}$ collisions at $\sqrt{\mathrm{S}_{\mathrm{NN}}}=200 \mathrm{GeV}$ 
The exact $\left\langle\beta_{\mathrm{T}}\right\rangle$ value that corresponds to a certain energy can be extracted from the combined fit to identified hadron spectra using a blast-wave model [25]. The blast-wave model assumes that particles produced in the collision are locally thermalized and move with a common transverse collective flow velocity $<\beta_{\mathrm{T}}>$ undergoing an instantaneous common freeze-out at a kinetic freeze-out temperature. As an example, results of such fits to particle spectra at $0-5 \%$ centrality class from Figure 1.5, as well as the rest of centrality classes at both LHC and RHIC energies are shown together in Figure 1.6 as a function of particle multiplicity. It can be seen that in both cases the value of $\left\langle\beta_{\mathrm{T}}\right\rangle$ increases with increasing multiplicity, or, in terms of centrality, becomes larger for more central collisions, which is consistent with the expectation of a more rapid QGP expansion for head-on collisions.

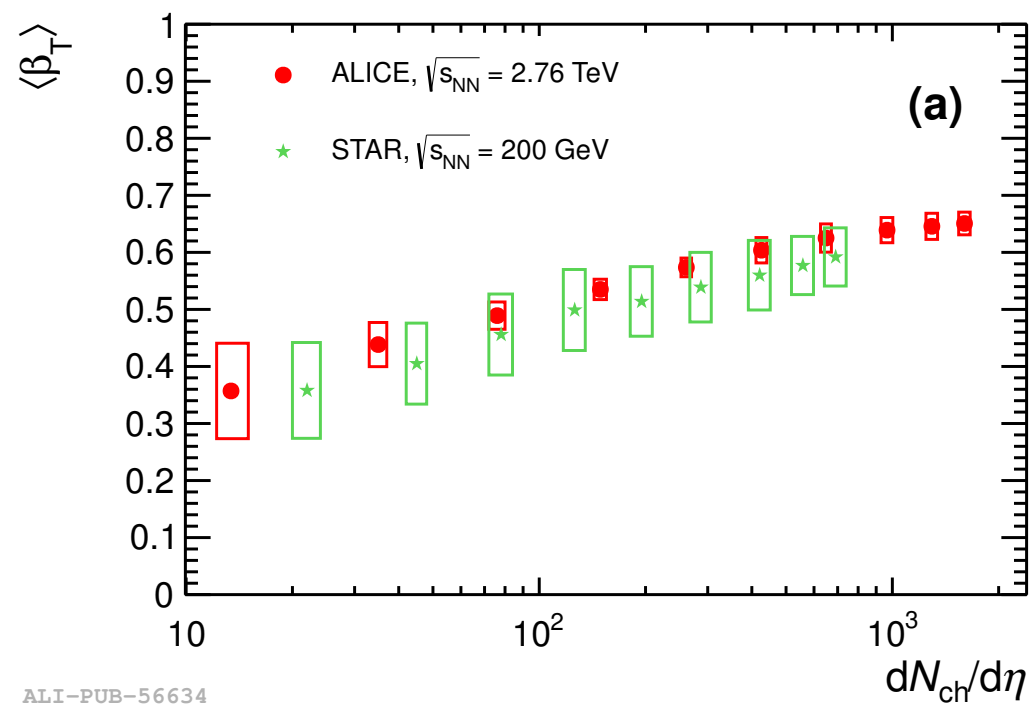

FiguRE 1.6: Blast-wave fit parameter $\left\langle\beta_{\mathrm{T}}>\right.$ as a function of $\mathrm{dN}_{\mathrm{ch}} / \mathrm{d} \eta$ extracted from ALICE $\mathrm{Pb}-\mathrm{Pb}$ data at $\sqrt{\mathrm{s}_{\mathrm{NN}}}=2.76 \mathrm{TeV}$ compared to STAR results in $\mathrm{Au}-\mathrm{Au}$ collisions at $\sqrt{\mathrm{S}_{\mathrm{NN}}}=200 \mathrm{GeV}$

In case of an asymmetric non-central collision, the shape of the overlapping zone between two colliding nuclei becomes closer to elliptic, as shown in the middle part of Figure 1.3. Such initial spatial eccentricity leads to a larger pressure gradient in the $\mathrm{x}$ than in $\mathrm{y}$ direction that, in turn, leads to an asymmetric expansion of the QGP medium. The QGP transport properties characterised by shear and bulk viscosities define the further development of such gradients. While bulk viscosity acts against the buildup of radial flow, i.e. affects the radial expansion of the matter, shear viscosity governs the rate of momentum transfer in the presence of inhomogeneity of fluid velocity, i.e. affects the anisotropic expansion. In general, higher shear viscosity should lead to stronger friction between 
fluid layers and will, therefore, cause the dampening of spatial gradients. A detailed comparison between experimental data and hydrodynamic predictions, performed previously, has revealed that the shear viscosity to entropy density ratio of the QGP is close to probably the smallest value that has ever been observed [26], i.e. $\eta / \mathrm{s} \sim 1 / 4 \pi$. This led to the conclusion that instead of behaving as a weakly interacting gas of quarks and gluons, the QGP behaves as a strongly coupled, nearly perfect liquid. Therefore, the aforementioned spatial anisotropy is not washed out, but is converted into the final state azimuthal anisotropy in momentum space [27]. This feature is called anisotropic flow.

In addition to the initial spatial anisotropy, the geometry of the overlap region in each event is affected by initial fluctuations of participating nucleons, introduced in Section 1.2.1, which might give rise not only to the elliptic configuration, but also various other geometry configurations contributing to the overall shape (triangular, rectangular and so on). This way, the shape of the overlap region in peripheral events turns out to be not perfectly elliptic, but rather irregular. Therefore, the momentum distribution of final state hadrons reflects a superposition of all different geometry configurations present since the beginning of the collision. To characterize these various patterns of anisotropic flow experimentally, a Fourier decomposition of the event averaged azimuthal particle distribution is used [28]:

$$
\frac{d N}{d \phi} \propto 1+2 \sum_{n=1}^{\infty} v_{n} e^{i n\left(\varphi-\Psi_{n}\right)}
$$

where

$$
v_{n}=\left\langle\operatorname{cosn}\left(\varphi-\Psi_{n}\right\rangle\right)
$$

are the flow coefficients corresponding to a $\mathrm{n}^{\text {th }}$-order symmetry plane angle $\Psi_{\mathrm{n}}$, $\varphi$ is the azimuthal angle of the particle of interest and the brackets denote an average over selected particles and events. This way, the second Fourier coefficient $\mathrm{v}_{2}$ is associated with the second order spatial eccentricity, i.e. the elliptic deformation of the created fireball (ellipticity) and is referred to as elliptic flow, while the third coefficient $\mathrm{v}_{3}$ - with the third order eccentricity, i.e. the triangular deformation (triangularity) and is referred to as triangular flow and so on. The second Fourier coefficient, $v_{2}$ or elliptic flow, is the dominant harmonic and reflects the initial elliptic geometry of the collisions. Therefore, $\mathrm{v}_{2}$ is expected to increase towards more peripheral collisions where the elliptic shape becomes more pronounced. Since symmetry planes are not accessible in the experiment, 
$\mathrm{v}_{\mathrm{n}}$ coefficients are estimated using two-particle [29], [30] or multi-particle correlations calculated with cumulants technique [31], [32], [33].

Two-particle correlations, apart from direct calculations of flow harmonics, are commonly studied in a qualitative way using the two-particle angular correlation function. An example of this observable measured by the ALICE experiment in central $\mathrm{Pb}-\mathrm{Pb}$ collisions is presented in Figure 1.7 [34]. Few distinct structures can be observed: the peak around $(\Delta \eta, \Delta \varphi)=(0,0)$ corresponding to a contribution from particle pairs originating from the same jet, the elongated structure around $\Delta \varphi \sim \pi$ (away side) that partly belongs to pairs of particles from backto-back jets and the structure around $\Delta \varphi \sim 0$ (near side) extending up to larger values in $\Delta \eta$, called near-side ridge. The latter is attributed to anisotropic flow which leads to a larger amount of particles travelling close together with $\Delta \varphi \approx 0$ than with $\Delta \varphi \approx \pi / 2$. This results in a larger amount of particle pairs with a small difference in $\Delta \varphi$ along all $\Delta \eta$ values. Therefore, the near-side ridge, which indicates that particles share long-range correlations, together with correlations coming from particle pairs located on the away side but not originating from back-to-back jets, is interpreted as a consequence of the collective expansion of the QGP medium in heavy-ion collisions. Quantitatively, both of these structures can be described by the combination of the flow coefficients from Equation 1.2 with the dominant contribution coming from elliptic flow [35].

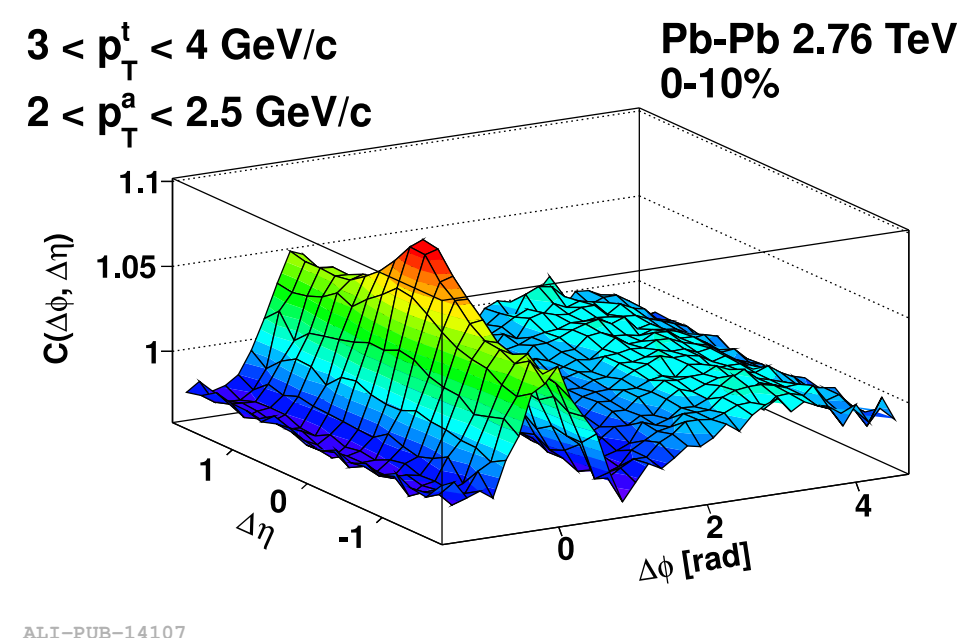

Figure 1.7: Two-dimensional two-particle correlation function $\mathrm{C}(\Delta \varphi, \Delta \eta)$ in central $\mathrm{Pb}-\mathrm{Pb}$ collisions at $\sqrt{\mathrm{s}_{\mathrm{NN}}}=2.76 \mathrm{TeV}$

A remarkable success of hydrodynamics in reproducing results of aforementioned observables is demonstrated in Figure 1.8 [30], where $\mathrm{v}_{2}$, obtained with twoparticle correlations, is presented for various hadron species in $\mathrm{Pb}-\mathrm{Pb}$ collisions for $20-30 \%$ centrality class and is compared to predictions from the hybrid iEBEVISHNU model [36]. In the data, the so-called mass ordering as a function of 
$\mathrm{p}_{\mathrm{T}}$ is seen. Heavier particles have smaller values of $\mathrm{v}_{2}$ compared with lighter ones, which is understood as a consequence of the interplay between radial and anisotropic flow [30]. In the model, the hydrodynamic description of the QGP evolution is coupled to a simulation of the hadronic rescattering phase. The shear viscosity to entropy density ratio parameter is chosen to be $\eta / \mathrm{s}=0.08$. A very good agreement between experimental results and corresponding model predictions is observed.

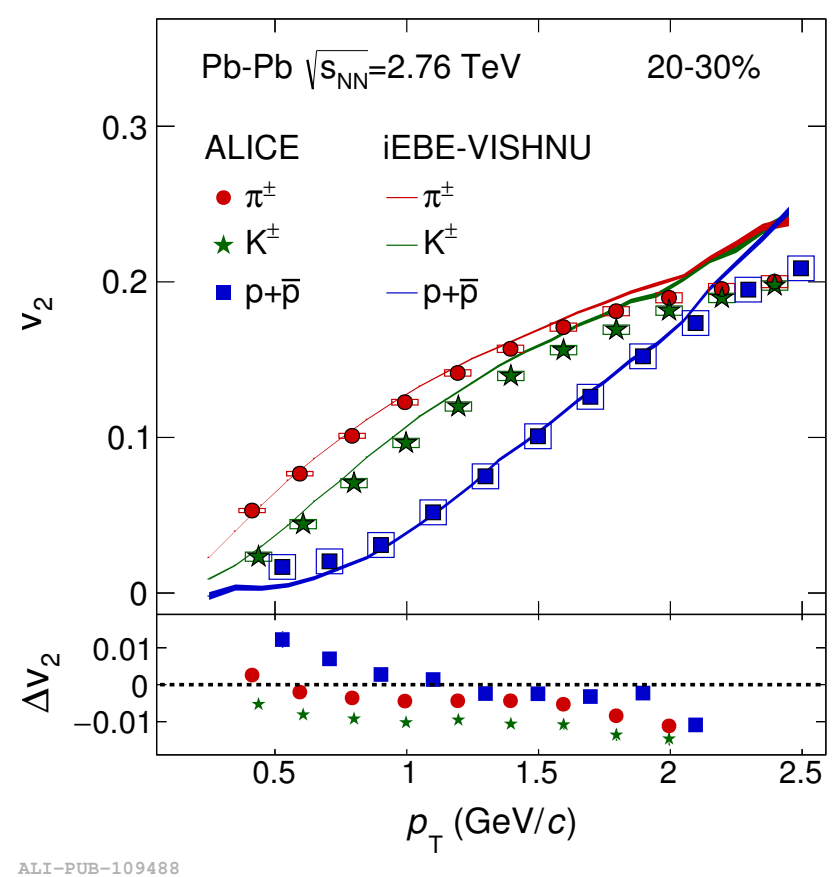

FIGURE 1.8: $\mathrm{v}_{2}$ results as a function of $\mathrm{p}_{\mathrm{T}}$ obtained with two-particle correlations for pions, kaons and protons in 20-30\% centrality class in $\mathrm{Pb}-\mathrm{Pb}$ collisions at $\sqrt{\mathrm{s}_{\mathrm{NN}}}=2.76 \mathrm{TeV}$. Comparison with hydrodynamic calculations from iEBE-

VISHNU model is also presented

Another way of measuring $\mathrm{v}_{2}$ and the rest of the flow coefficients is by using multiparticle correlations extracted with cumulants. Since collectivity is expected to be shared between many particles, the observation of non-zero cumulants and corresponding flow harmonics suggests the presence of long-range multiparticle correlations and is, therefore, interpreted as an evidence for a collectively expanding system. One example of such observations is shown in Figure 1.9 which presents the results of four-particle cumulant $\mathrm{c}_{2}\{4\}$ extracted in $\mathrm{Pb}-\mathrm{Pb}$ collisions at $\sqrt{\mathrm{S}_{\mathrm{NN}}}=5.02 \mathrm{TeV}$. Different data points correspond to different methods used to suppress the contribution from correlations that do not originate from collective effects, e.g. the one generated by jets or resonance decays. In order to extract a real-valued $\mathrm{v}_{2}\{4\}$ from $\mathrm{c}_{2}\{4\}$, the latter should have a negative sign due to the relation $\mathrm{v}_{\mathrm{n}}\{4\}=\sqrt[4]{-\mathrm{c}_{\mathrm{n}}\{4\}}$. 


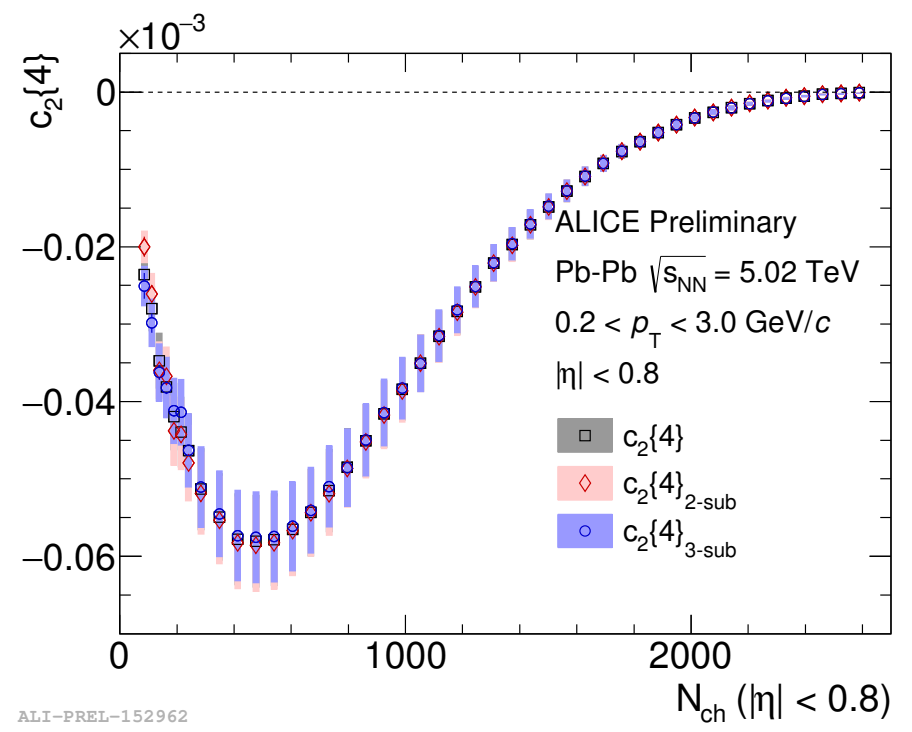

Figure 1.9: Multiplicity dependence of the four-particle cumulant $c_{2}\{4\}$ in $\mathrm{Pb}-\mathrm{Pb}$ collisions at $\sqrt{\mathrm{SNN}}=5.02 \mathrm{TeV}$

Collective properties of the QGP, using the aforementioned "bulk" observables, are usually probed in the regime where particles have low transverse momenta and participate in the common expansion of the source, i.e. the so-called "bulkdominated" regime. On the contrary, particles with high transverse momenta, predominantly originating from parton fragmentation that leads to jet formation, belong to the "jet-dominated" regime. Since they quickly pass through the medium produced in relativistic nuclear collisions, they do not participate in the collective motion of the bulk. However, when jets propagate through the hot and dense medium, due to multiple scatterings with the surrounding partons but also due to the emission of radiation they lose energy. This should result in a suppression of high transverse momentum particles in nucleus-nucleus (A-A) with respect to proton-proton (pp) collisions, where the QGP medium is not expected to form. This phenomenon is called "jet quenching". One of the most frequently used observables for studying modifications in measured high transverse momentum spectra is the nuclear modification factor $R_{\mathrm{AA}}$.

The nuclear modification factor quantifies the modification of high $\mathrm{p}_{\mathrm{T}}$ particle production in heavy-ion collisions with respect to pp collisions using the ratio of the corresponding $\mathrm{p}_{\mathrm{T}}$ spectra $\mathrm{dN}_{\mathrm{AA}} / \mathrm{dp}_{\mathrm{T}}$ and $\mathrm{dN}_{\mathrm{pp}} / \mathrm{dp}_{\mathrm{T}}$ scaled up to account for the different size between protons and ions, according to:

$$
R_{A A}=\frac{d N_{A A} / d p_{T}}{\left\langle N_{c o l l}\right\rangle d N_{p p} / d p_{T}} .
$$


The value of $R_{A A}$ is expected to be equal to 1 in a scenario where no in medium modification is present. In this case heavy-ion collisions can be considered as a superposition of many individual pp collisions and particle production is not modified in the first with respect to the second case. If, instead, $\mathrm{R}_{\mathrm{AA}}$ is smaller than 1, this would indicate a suppression of the particle production in A-A collisions which is attributed to the QGP creation [37].

An example of a nuclear modification factor measurement for charged particles in $\mathrm{Pb}-\mathrm{Pb}$ collisions at $\sqrt{\mathrm{S}_{\mathrm{NN}}}=2.76 \mathrm{TeV}$ is shown in Figure 1.10 [38]. Results are presented as a function of $\mathrm{p}_{\mathrm{T}}$ for most central (0-5\%) and most peripheral (70-80\%) collisions. The deviation from 1 can be seen in both cases which is consistent with a scenario of larger energy loss of high $\mathrm{p}_{\mathrm{T}}$ particles in $\mathrm{Pb}-\mathrm{Pb}$ case with respect to the pp case. However, while a significant suppression of $\mathrm{R}_{\mathrm{AA}}$ in central events is observed, only moderate change is seen in peripheral collisions. This is due to the fact that in the latter case partons have shorter average path lengths since the medium has smaller size, lifetime and density. This leads to an overall smaller energy loss in peripheral with respect to central collisions.

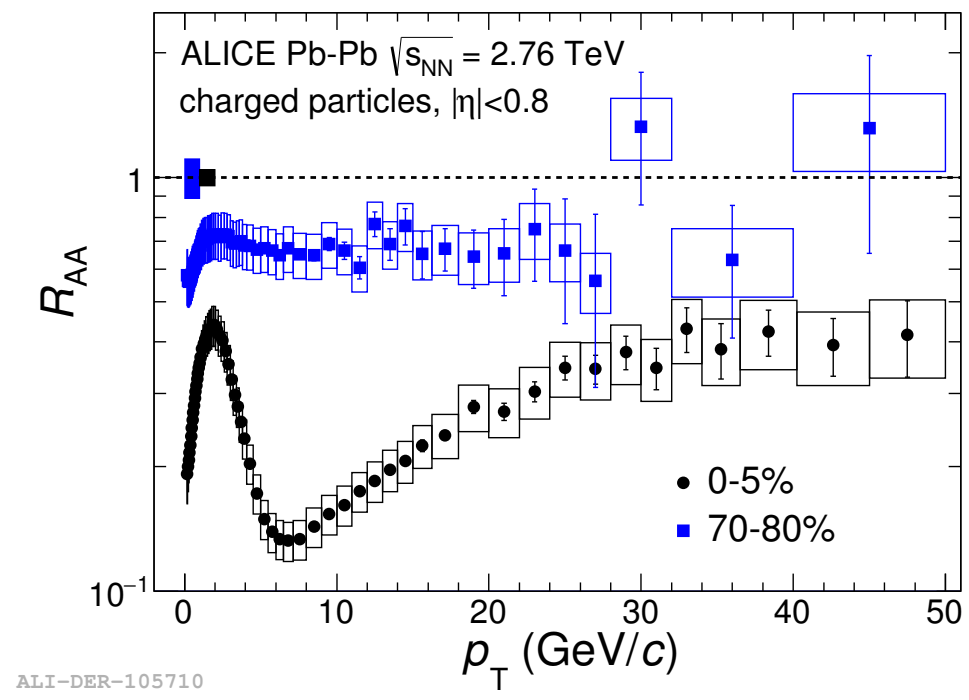

FiguRE 1.10: Nuclear modification factor of charged particles in central and peripheral $\mathrm{Pb}-\mathrm{Pb}$ collisions at $\sqrt{\mathrm{s}_{\mathrm{NN}}}=2.76 \mathrm{TeV}$

This way, the existence of collective motion in heavy-ion collisions (also denoted as large systems), driven by the formation of a hot and dense QGP medium with properties of a strongly coupled nearly perfect liquid, is confirmed by measurements of many observables used for studies both at low and high transverse momenta. Furthermore, an effective description of these effects in the bulk region provided by hydrodynamic models has been established and tested precisely. 
In proton-nucleus (p-A) or pp collisions, referred to as small systems, a common naive expectation of no QGP formation has been recently challenged by observations of effects that are attributed to the development of collectivity in A-A systems. Currently, it is still not fully understood what is the origin behind these effects and if the origin is similar to the one in large systems. The next section presents a very brief status of our present knowledge of collective phenomena in small systems.

\subsection{Signs of collectivity in small systems}

Proton-nucleus and proton-proton collisions used to provide a reference for studies of observables discussed in Section 1.2.3. This was based on expectations that the QGP can not form in these collision systems due to the small system size. Surprisingly, recent studies in small systems revealed effects that point in the direction of collective motion present in high-multiplicity proton-lead ( $\mathrm{p}-\mathrm{Pb}$ ) and pp collisions [39], [40]. These unexpected results stressed the need to explore whether the QGP is still formed in the smallest hadronic systems.

The first observation that opened up the discussion about collective phenomena in small systems was the presence of the near-side ridge in two-particle angular correlations in high-multiplicity pp collisions at $\sqrt{\mathrm{s}}=7 \mathrm{TeV}$ reported by CMS in 2010 [39], shown in Figure 1.11. On the left, the two-dimensional two-particle correlation function $\mathrm{R}(\Delta \varphi, \Delta \eta)$ is presented for charged particles in the $\mathrm{p}_{\mathrm{T}}$ range of $1 \mathrm{GeV} / \mathrm{c}<\mathrm{p}_{\mathrm{T}}<3 \mathrm{GeV} / \mathrm{c}$ in minimum bias events (no multiplicity selection applied). On the right, similar result is presented for high-multiplicity events. The minimum-bias distribution shows the typical expected structures, similar to the ones observed in $\mathrm{Pb}-\mathrm{Pb}$ collisions, such as the contribution from same jets near $(\Delta \eta, \Delta \varphi)=(0,0)$ or broad elongated ridge around $\Delta \varphi=\pi$ due to back-toback jets fragmentation. No significant correlation is observed around $\Delta \varphi=0$ and large $\Delta \eta$ values. However, in high-multiplicity events an unexpected nearside ridge extending to large values of $\Delta \eta$, interpreted as a consequence of the development of anisotropic flow in heavy-ion collisions, is seen. 
(b) CMS MinBias, $1.0 \mathrm{GeV} / \mathrm{c}<\mathrm{p}_{\mathrm{T}}<3.0 \mathrm{GeV} / \mathrm{c}$

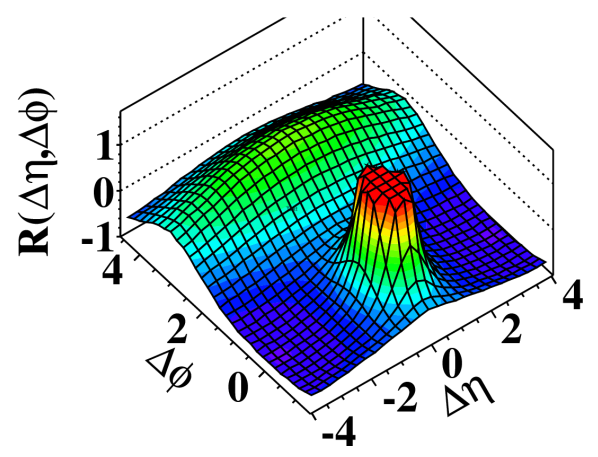

(d) CMS N $\geq 110,1.0 \mathrm{GeV} / \mathrm{c}<\mathrm{p}_{\mathrm{T}}<3.0 \mathrm{GeV} / \mathrm{c}$

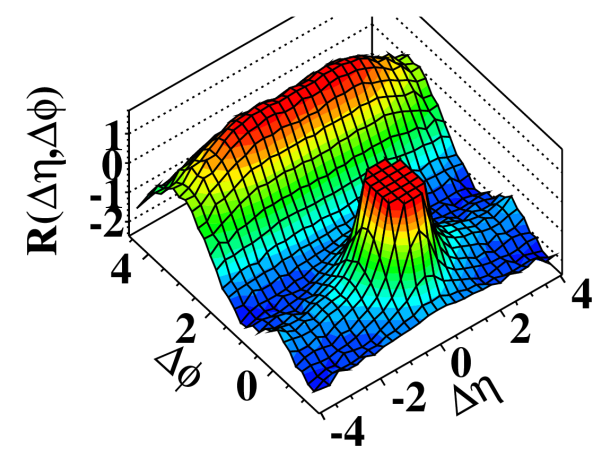

FiguRE 1.11: Two-dimensional two-particle correlation function $\mathrm{R}(\Delta \varphi, \Delta \eta)$ in low-multiplicity (left) and high-multiplicity (right) pp collisions at $\sqrt{\mathrm{s}}=7 \mathrm{TeV}$

After this, a similar behaviour was discovered in $\mathrm{p}-\mathrm{Pb}$ collisions at $\sqrt{\mathrm{s}_{\mathrm{NN}}}=$ $5.02 \mathrm{TeV}$ [40]. Figure 1.12 shows the two-dimensional per-tigger yield for charged particles in the same momentum range as in the aforementioned analysis in pp collisions. The results for low- and high-multiplicity events are presented on the left and on the right, respectively. While correlations originating from same and back-to-back jets are seen in both distributions, a significant ridge structure at $\Delta \varphi=0$ over all $\Delta \eta$ bins is observed only in the high-multiplicity case.
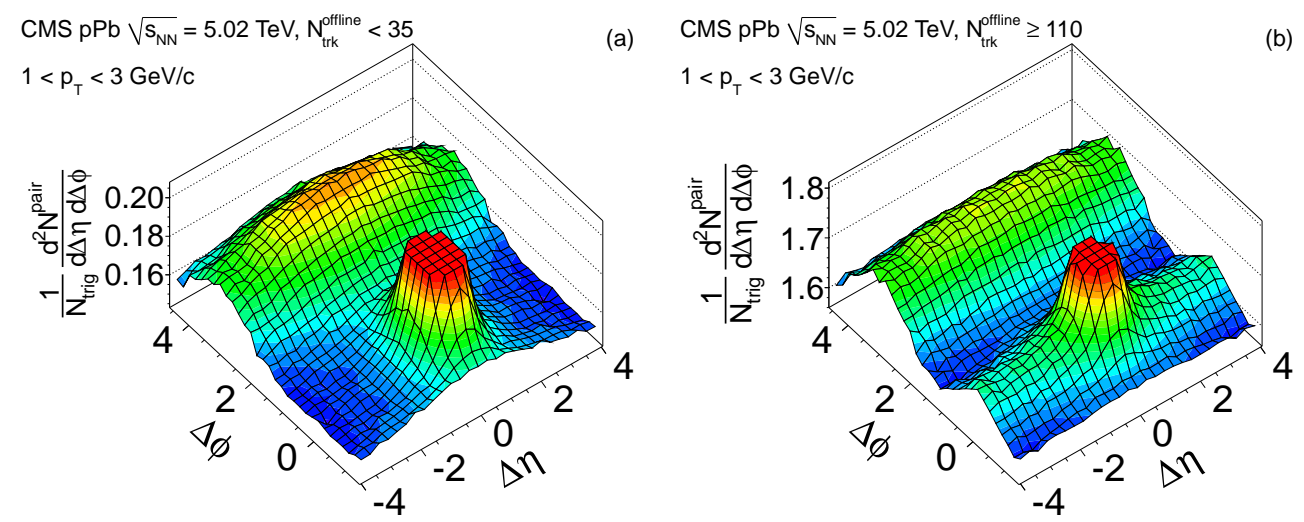

FIGURE 1.12: Two-dimensional per-trigger yield in low-multiplicity (left) and high-multiplicity (right) $\mathrm{p}-\mathrm{Pb}$ collisions at $\sqrt{\mathrm{s}_{\mathrm{NN}}}=5.02 \mathrm{TeV}$

Later on, observations of long-range correlations in $\mathrm{p}-\mathrm{Pb}$ and $\mathrm{pp}$ collisions were confirmed by ALICE, ATLAS and LHCb experiments [41]. In addition, the PHENIX collaboration reported the presence of the ridge in very central deuterongold $(\mathrm{d}-\mathrm{Au})$ collisions [42]. This triggered many followup experimental measurements, as well as theory developments, with the purpose of finding the underlying physics mechanisms behind the ridge in small systems. 
This structure has been extensively analyzed in a quantitative way using the Fourier decomposition into flow coefficients (see Section 1.2.3 for more details). To understand whether these angular correlations originate only from two particles or are shared between many particles, the multi-particle cumulant technique has been widely applied. Flow measurements up to four-particle cumulants were performed in p-Pb collisions by ATLAS [43], CMS [44] and ALICE [45] experiments. In these studies, similar behaviour to the one present in heavy-ion collisions was found, such as the negative sign of four-particle cumulant $\mathrm{c}_{2}\{4\}$ introduced previously in Section 1.2.3. Contrary to $\mathrm{p}-\mathrm{Pb}$, in $\mathrm{pp}$ collisions the extraction of the negative $c_{2}\{4\}$ and the subsequent extraction of the real-valued $\mathrm{v}_{2}\{4\}$ is a challenging task due to the large contribution of so-called non-flow effects. However, in one of the most recent and extensive studies done by ALICE [46], real-valued $\mathrm{v}_{2}\{4\}$ was extracted for the first time in pp collisions. In analogy to the case of heavy-ion collisions, such observations suggest the presence of long-range multi-particle correlations driven by the collective motion of the QGP and, therefore, can be interpreted as a confirmation of a hydrodynamically expanding medium in small systems.

Another observation that is in favor of such interpretation is the fact that the identified particle spectra in $\mathrm{p}-\mathrm{Pb}$ and $\mathrm{pp}$ collisions show the same characteristic features as in $\mathrm{Pb}-\mathrm{Pb}$ collisions (see Figure 1.5), in particular a significant hardening with increasing multiplicity more pronounced for heavier hadrons [47], [48]. Since this dependence in heavy-ion collisions is explained as a consequence of radial flow being larger in central with respect to peripheral collisions, such findings in small systems are consistent with the idea of a larger radial flow in high with respect to low multiplicity events. Furthermore, particle ratios such as $\mathrm{p} / \pi$ and $\Lambda / \mathrm{K}_{\mathrm{S}}^{0}$ in p-Pb and pp collisions [47], [49] show qualitatively similar enhancement at intermediate $\mathrm{p}_{\mathrm{T}}$ attributed, as one of the possibilities, to radial flow effects in $\mathrm{Pb}-\mathrm{Pb}$ collisions [50]. In addition, an enhancement in strange hadron production as a function of charge particle multiplicity, originally considered as one of QGP signatures in nuclear collisions [51], has also been recently observed in $\mathrm{p}-\mathrm{Pb}$ [52], [53] and pp collisions [54].

Apart from the aforementioned observables, also the flow coefficients have been extensively studied for identified hadrons. In heavy-ion collisions, a mass ordering effect in $\mathrm{v}_{\mathrm{n}}$ results as a function of $\mathrm{p}_{\mathrm{T}}$ is observed, where heavier particles have smaller values of $\mathrm{v}_{\mathrm{n}}$ compared to lighter ones at $\mathrm{p}_{\mathrm{T}}<2-3 \mathrm{GeV} / \mathrm{c}$ [30], [55]. This effect is understood as a consequence of the interplay between radial and anisotropic flow [30]. Similar ordering has been found in both p-Pb [56], [57] and pp [58] collisions, which is also consistent with hydrodynamic picture and is in agreement with hydrodynamic calculations [59], [60]. In addition, a significant $\mathrm{v}_{2}$ 
signal from long-range azimuthal correlations is observed for charm and strange hadrons in high-multiplicity p- $\mathrm{Pb}$ collisions [61] and muons from charm-hadron decays in pp collisions [62]. These results indicate that heavy-flavour quarks participate in the collective expansion like light quarks in high multiplicity $\mathrm{p}-\mathrm{Pb}$ and pp collisions.

Furthermore, the hydrodynamic paradigm was tested successfully in a study of the flow coefficients in proton-gold (p-Au), deuteron-gold (d-Au), and heliumgold $\left({ }^{3} \mathrm{He}-\mathrm{Au}\right)$ collisions performed by PHENIX experiment at $\sqrt{\mathrm{s}}=200 \mathrm{GeV}[63]$. Varying these collision systems from $\mathrm{p}-\mathrm{Au}$, to d-Au, to ${ }^{3} \mathrm{He}-\mathrm{Au}$ changes the initial geometry from dominantly circular, to elliptical and to triangular configurations, respectively. Given the presence of collective expansion, spatial ellipticity and triangularity should be transformed into significant $\mathrm{v}_{2}$ and $\mathrm{v}_{3}$ flow coefficients, respectively (see Section 1.2.3 for more details on this relation). A clear prediction can be made for the ordering of $\mathrm{v}_{2}$ and $\mathrm{v}_{3}$ values in these systems that one should obtain in hydrodynamic models, based on the relation between spatial eccentricities and final momentum anisotropies, namely $v_{2}^{\mathrm{p}-\mathrm{Au}}<\mathrm{v}_{2}^{\mathrm{d}-\mathrm{Au}} \approx \mathrm{v}_{3}^{3} \mathrm{He}-\mathrm{Au}$ and $\mathrm{v}_{3}^{\mathrm{p}-\mathrm{Au}} \approx \mathrm{v}_{3}^{\mathrm{d}-\mathrm{Au}}<\mathrm{v}_{3}^{3} \mathrm{He}-\mathrm{Au}$. The comparison of the data with predictions from two hydrodynamic models shows a very good agreement which suggests that in small systems the $\mathrm{v}_{\mathrm{n}}$ coefficients are also strongly correlated to the initial geometry.

However, some caveats exist regarding the hydrodynamic description of the data in small systems. In the case of $\mathrm{p} / \mathrm{d}-\mathrm{A}$ or pp collisions, contrary to large systems, the applicability of hydrodynamics seems to be significantly dependent on assumptions about the nature of the initial state discussed previously in Section 1.2.1. While the initial state description, where fluctuations at the level of nucleons are considered, successfully works for heavy-ion collisions, for small systems the inclusion of sub-nucleonic fluctuations, described in terms of color correlations computed in the color glass condensate effective field theory (CGC EFT) [64], was found to become much more important [65]. In fact, results of flow coefficients presented in the aforementioned paper were even first claimed to be fully reproduced only in terms of color correlations, i.e. solely by initial state with no final state hydrodynamic evolution involved in the simulation [66]. However, due to a numerical error found later in the presented calculations, that led to a change in the final results, this statement was withdrawn. It was clearly shown that the initial state only can not describe results of $v_{n}$ seen in the data and, thus, the hydrodynamic component is essential [67]. This way, most successful recent models for small systems incorporate both stages, but in some cases even with the inclusion of sub-nucleonic fluctuations they are not able to reproduce the data. For example, while the hybrid hydrodynamic model iEBE-VISHNU 
successfully describes the integrated and differential $\mathrm{v}_{2}$ results from two-particle correlations for all charged and identified hadrons in $\mathrm{p}-\mathrm{Pb}$ and $\mathrm{Pb}-\mathrm{Pb}$ systems, it is not able to reproduce the negative sign of $c_{2}\{4\}$ measured in pp collisions [68]. The authors argue that this could be caused by the limited understanding of the proton initial state. Another example is the IP-Glasma+MUSIC+UrQMD model [69] that gives a very good agreement to multiplicity and flow distributions at RHIC and LHC [70], but is still not able to reproduce multi-particle measurements in pp collisions reported in [46]. Therefore, a clear understanding of initial state effects in small systems required for a successful description of the data by hydrodynamic models is still missing.

Some caveats also remain on the experimental side. One of the biggest unsolved puzzles is connected to the lack of jet quenching effects in small collision systems. As mentioned previously in Section 1.2.3, an important confirmation of the QGP formation in heavy-ion collisions is provided by the presence of jet quenching. In small systems, since the majority of bulk observables suggests the creation of a QGP medium, it would be reasonable to expect the presence of parton energy loss within the medium there as well. However, no jet quenching effects were observed in $\mathrm{d}-\mathrm{Au}$ or $\mathrm{p}-\mathrm{Pb}$ collisions [71], [72], [73], [74] contrary to $\mathrm{Pb}-$ $\mathrm{Pb}$ collisions [38], [75]. One possible explanation for these observations is that in small systems, due to the shorter lifetime of the medium compared with heavy-ion collisions, the parton evolution is much less affected by the scattering with other surrounding partons. Nevertheless, it is clear that more studies are necessary in order to explain the simultaneous presence of anisotropic flow for charged and identified hadrons, including heavy flavour species, and absence of jet quenching effects in small systems.

Moreover, a number of alternative physics mechanisms that lead to the final state momentum anisotropy without involving the QGP formation have been proposed. These include momentum correlations generated at the earliest stages of the collision, elastic parton-parton scattering and color reconnection. The models that use the first approach are referred to as initial-state momentum correlation models, while the ones that employ the second approach - parton transport models. The CGC-based framework, introduced earlier in the text, is one of the examples belonging to the first category. It is able to describe many features of two-particle correlations in pp and p-Pb collisions [76], [77], but as already pointed out, it can not reproduce the ordering of $\mathrm{v}_{\mathrm{n}}$ results. Elastic parton-parton scattering is employed in parton transport models such as A Multi-Phase Transport (AMPT) model [78]. In AMPT the produced partons are allowed to scatter, recombine into hadrons via coalescence and undergo hadronic inelastic and elastic scattering. Azimuthal anisotropies are produced not by the 
pressure-driven collective flow, but mainly by the anisotropic parton escape probability as a response to the initial spatial eccentricity through interactions [79]. The AMPT model, in particular the string melting tune, was shown to be often able to successfully reproduce qualitative and even quantitative features of most of experimental observables, such as particle spectra, long-range azimuthal correlations and flow harmonics, not only in heavy-ion collisions [13], [79], but also in $\mathrm{p}-\mathrm{Pb}$ and pp collisions [80]. Therefore, parton transport models remain one of possible alternatives for the perfect liquid paradigm not only in small systems, but even in A-A systems. Color reconnection mechanism implemented, for example, in PYTHIA [81] and DYPSI [82] models is based on multi-parton interactions [83]. It allows for the interaction between color strings which leads to a flow-like patterns in the measured observables. So far it has been tested only in pp collisions and it was found, for example, to be able to describe qualitatively the features of the data for ratios of identified hadron yields driven by radial flow in heavy-ion collisions [84].

This way, currently there is no common theoretical framework that can provide a simultaneous description of the wealth of experimental data available in small systems. The origin of signs of collectivity there remains a highly debated topic over whether these effects are of hydrodynamic origin or are caused by other alternative mechanisms not connected to the creation of a QGP medium. The goal of this thesis is to study the origin of these collective effects observed in pp collisions, using charged-dependent angular correlations. The latter will be done with the balance function, introduced and discussed in the following chapter, which has been established as a sensitive tool to study the collective motion of the system. 



\section{Chapter 2}

\section{The balance function}

Particle production in any process, and thus also in collisions between nuclei, is governed by conservation laws. One of these laws prescribes that particles, no matter if hadrons or quarks, are created in pairs of opposite electric charge i.e. local charge conservation. These pairs of particles, called from now on "balancing partners", are strongly correlated in coordinate space since they are produced at the same location in space and time. In heavy-ion collisions, the creation of such pairs at the level of quarks mostly occurs during the expansion of the quark-gluon plasma. Because of this, the initial spatial correlation is affected by the properties of this medium. This way, studying the degree of the final state charge-dependent correlations allows probing different aspects of the QGP evolution such as the hadronization time, freeze-out conditions and the collective motion. The experimental tool that allows measuring these correlations is the balance function described in Section 2.1.

The final state correlations are reflected in the balance function width which, as explained in Section 2.2.1, is particularly sensitive to the radial flow and the creation time of the balancing charges in heavy-ion collisions [85]. In addition, the balance function measured for different particle species in A-A systems provides an important insight to the chemical evolution of the QGP, i.e. when different quark and hadron flavours are created and how they are balanced [86].

However, balance function measurements are of great interest not only in heavyion collisions, but also in collisions between the small systems such as p-A and pp, where a QGP medium was initially not expected to form. An open question about the origin of collectivity, signs of which were recently observed in small systems, as described in Section 1.3, makes a comparison of balance functions measured in heavy-ion collisions to those measured in small systems of special 
importance. The details of what the measurement of the balance function brings in $\mathrm{p}-\mathrm{A}$ or pp collisions are discussed in Section 2.2.2.

Finally, quantitative information about the properties of the created system, as well as the particle production mechanisms in different collision types, can be extracted from the comparison of experimental data with model predictions. Expectations for balance function measurements from different models can be found in Section 2.3, while previous experimental measurements are described in Section 2.4.

\section{$2.1 \quad$ Definition}

The balance function, proposed by Pratt, Bass and Danielewicz [87], is defined according to

$$
B\left(p_{2} \mid p_{1}\right)=\frac{1}{2}\left\{\rho\left(\beta, p_{2} \mid \alpha, p_{1}\right)-\rho\left(\beta, p_{2} \mid \beta, p_{1}\right)+\rho\left(\alpha, p_{2} \mid \beta, p_{1}\right)-\rho\left(\alpha, p_{2} \mid \alpha, p_{1}\right)\right\},
$$

where

$$
\rho\left(\beta, p_{2} \mid \alpha, p_{1}\right)=\frac{N\left(\beta, p_{2} \mid \alpha, p_{1}\right)}{N\left(\alpha, p_{1}\right)}
$$

is the conditional probability of finding a particle of type $\beta$ in a bin $\mathrm{p}_{2}$ given the existence of a particle of type $\alpha$ in a bin $\mathrm{p}_{1}$. It is calculated by finding the number of particle pairs $\mathrm{N}\left(\beta, \mathrm{p}_{2} \mid \alpha, \mathrm{p}_{1}\right)$ in the same event that satisfy both conditions and dividing it by the number of particles $\alpha$, denoted as $\mathrm{N}\left(\alpha, \mathrm{p}_{1}\right)$, satisfying the first condition. As an example, $\mathrm{p}_{1}$ can refer to any momentum range, while $\mathrm{p}_{2}$ to the relative pseudorapidity $\Delta \eta=\eta_{2}-\eta_{1}$ between the two particles. Labels $\alpha$ and $\beta$ can indicate any type of conserved quantum number such as electric charge, baryon number, strangeness or charm content. For example, $\alpha$ can be associated to all negatively, while $\beta$ to all positively charged hadrons, or $\alpha$ to all hadrons containing a strange quark, while $\beta$ to all hadrons with an antistrange quark.

The analysis presented in this thesis focuses on the electric charge balance functions with $\mathrm{p}_{2}$ defined as a two-dimensional space given by $\Delta \eta$ and $\Delta \varphi$, where $\Delta \varphi=\varphi_{2}-\varphi_{1}$ is the relative azimuthal angle between any two particles. The balance function definition using Equation 2.2 with $\alpha$ referring to a negative particle and $\beta$ to a positive particle is then rewritten as: 


$$
\begin{gathered}
B(\Delta \eta, \Delta \varphi)=\frac{1}{2}\left\{\frac{\left\langle N_{-+}(\Delta \eta, \Delta \varphi)\right\rangle-\left\langle N_{++}(\Delta \eta, \Delta \varphi)\right\rangle}{\left\langle N_{+}\right\rangle}+\right. \\
\left.+\frac{\left\langle N_{+-}(\Delta \eta, \Delta \varphi)\right\rangle-\left\langle N_{--}(\Delta \eta, \Delta \varphi)\right\rangle}{\left\langle N_{-}\right\rangle}\right\}
\end{gathered}
$$

In the previous equation, the terms $\left\langle\mathrm{N}_{-+}\right\rangle,\left\langle\mathrm{N}_{+-}\right\rangle,\left\langle\mathrm{N}_{++}\right\rangle$and $\left\langle\mathrm{N}_{--}\right\rangle$are represented by two-dimensional distributions and are calculated by counting the number of corresponding particle pairs in a given $(\Delta \eta, \Delta \varphi)$ range in every event. After this, an average over all events is taken, represented by the brackets. Similar procedure is used to obtain $\left\langle\mathrm{N}_{+}\right\rangle$and $\left\langle\mathrm{N}_{-}\right\rangle$which both reflect single particle distributions. All details concerning such procedure applied in this analysis can be found in Section 4.3. The subtraction of like-sign terms is used to remove the same-charge correlations and statistically isolate the charge-dependent part, i.e. the balancing partners. The measured balance function therefore represents a probability of these particles being separated by $\Delta \eta$ and $\Delta \varphi$.

In the case of a perfectly efficient detector, that is able to reconstruct all balancing partners within the acceptance, the balance function should integrate to unity. This condition introduces a normalization factor of $1 / 2$ in front of Equation 2.3 that then yields:

$$
\begin{aligned}
\int_{r_{1}}^{r_{2}} B(\Delta \eta, \Delta \varphi) d \Delta \eta d \Delta \varphi & =\frac{1}{2}\left\{\frac{N_{-} \cdot N_{+}}{N_{-}}-\frac{N_{-} \cdot\left(N_{-}-1\right)}{N_{-}}+\frac{N_{+} \cdot N_{-}}{N_{+}}-\right. \\
\left.-\frac{N_{+} \cdot\left(N_{+}-1\right)}{N_{+}}\right\} & =\frac{1}{2}\left\{N_{+}-\left(N_{-}-1\right)+N_{-}-\left(N_{+}-1\right)\right\}=1,
\end{aligned}
$$

where $r_{1}$ and $r_{2}$ reflect the lower and upper limits of an integration range defined by the detector's acceptance in $\Delta \eta$ and $\Delta \varphi$.

One of the quantities that provide valuable information about the dynamics of the balancing charges is the width of the balance function, one of the main focus topics in this thesis. In addition, information on the number of correlated particle pairs as well as the chemical composition of the final state can be extracted from the balance function yield. The details on the procedure applied to compute these quantities in this analysis can be found in Section 4.3. In the following section, the sensitivity of the width and the yield to different components of the collision evolution is discussed from the theoretical point of view. 


\subsection{Balance function width and yield}

\subsubsection{Heavy-ion collisions}

The width of the two-dimensional balance function distribution reflects the effect of different stages of the QGP evolution on the final separation between balancing charges. According to [85], the overall balance function width $\sigma_{\eta}$ consists of two components:

$$
\sigma_{\eta}^{2}=\sigma_{\text {therm }}^{2}+\sigma_{\text {flow }}^{2}
$$

In the previous equation, $\sigma_{\text {therm }}=\sqrt{(2 \mathrm{~T} / \mathrm{m})}$ is related to the thermal spreading with $\mathrm{T}$ being the temperature of the medium at the moment when the particles, i.e. quarks or hadrons, are created and $m$ being the mass of the particle. On the other hand, $\sigma_{\text {flow }}$ is driven by the charge separation due to the expansion of the medium as well as the interaction of particles with the surrounding medium. It is also affected by the diffusion effect to a lesser extent.

It is important to point out that a heavy-ion collision consists of a partonic phase, characterised by a strongly coupled QGP medium, and the subsequent hadronization followed by the hadronic rescattering phase, where final state hadrons continue interacting. Since quarks are not accessible directly in the experimental measurements, balance functions are constructed from hadrons. These balance functions reflect the picture of hadron correlations at the freeze-out temperature. The dependence on this temperature is represented by the thermal term $\sigma_{\text {therm }}$ which will decrease with time due to the cooling of the system and, therefore, will narrow the total balance function width.

On the other hand, the $\sigma_{\text {flow }}$ term might significantly depend not only on the separation between hadrons created during the hadronic rescattering, but also on the fact that the charge moved as a free quark before combining into a hadron, i.e. the separation between the constituents quarks of hadrons created in the partonic stage [85]. To explain this better, a pair of strange and anti-strange quarks produced during the QGP evolution is presented schematically in Figure 2.1 [88]. In this picture, the pair is created at two distinct moments, i.e. at around $\sim 1 \mathrm{fm} / \mathrm{c}$, denoted as early stage, and at a later stage that occurs just before hadronization at around 5-10 fm/c. It can be seen that the balancing partners created early are expected to separate further in rapidity in comparison to the same pair created at a later stage. This is caused by the fact that the first pair will be more affected by the expansion of the medium and the scattering 
before hadronization than the second. In addition, for a pair of quarks produced just before the hadronization, the effect of diffusion becomes negligible. Therefore, a stronger correlation should be observed for the balancing quarks created at a later stage originating partially from a smaller contribution of the $\sigma_{\text {flow }}$ term.

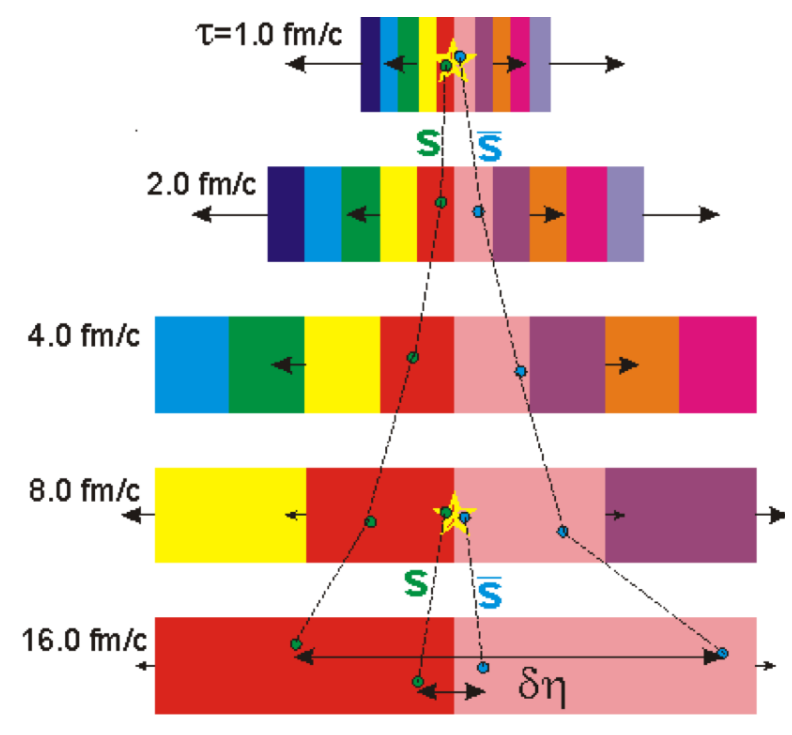

FIGURE 2.1: A schematic representation of early and late stage creation of the balancing partners

This way, balance functions can provide a meaningful information about the freeze-out conditions, the creation time and the mobility of constituent quarks. Apart from this, the separation between opposite quarks or hadrons will be affected by the degree of collective flow present in a heavy-ion collision. It is important to mention that, because of the subtraction of the like-sign correlations in Equation 2.3, the charge-independent anisotropic flow effects are cancelled out in the balance function measurement. However, the effect of radial flow, i.e. the velocity with which the system expands outwards, is still present and affects the balancing partners. The correlation between unlike-sign pairs created from the same neutral cluster becomes the only remaining component after the elimination of same-charge correlations. According to Equation 1.1, higher radial flow results in stronger momentum boost that every particle acquires. For a pair of opposite balancing charges, this will result in a tighter correlation in momentum space. This will be reflected in a narrower balance function distribution than in the case where no radial flow is present. The narrowing of the balance function width is therefore not only related to the later production of quarks, but also to the larger value of radial flow. Vice versa, the broadening of the balance function 
width would be a signal of an early production of quarks or a reduced radial flow.

Since both aforementioned components depend on collision geometry, reflected by the impact parameter, a centrality dependence of the balance function width is expected. In central heavy-ion collisions, where nuclei collide head-on, the created QGP has a bigger size and a longer lifetime of partonic stage with respect to the one in peripheral collisions, where only small parts of the nuclei overlap (see Section 1.2.2 for more details). The extended lifetime of the pre-hadronic stage could lead to a later hadronization which has been referred to as the delayed hadronization scenario [85]. Since most of the balancing charges in the QGP are expected to be produced at the hadronization [87], in this delayed hadronization scenario, they will be produced later. This should thus result in a tighter correlation between the balancing charges in the central than in peripheral collisions. In addition, radial flow is higher in central with respect to peripheral collisions (see Section 1.2.3). Therefore, both terms would lead to a narrowing of the balance function width for more central than more peripheral events.

At the same time, one should remember that after hadronization, the final state correlated hadrons might be pulled apart due to diffusion in the hadronic rescattering phase which will lead to an additional broadening of the width.

An important insight into the QGP properties can be gained not only from the studies of the balance function width, but also the yield. Such measurements for different hadronic species might help identifying the evolution of parton creation in the medium as well as the way quarks of different flavours balance each other, something usually referred to as the QGP chemistry [86]. The first component in this definition, i.e. the time component related to an early or later stage creation of quark pairs in the QGP evolution depending on their flavour, will affect the shape of the balance function and, hence, will be reflected more in its width. More detailed study of the effects of this component can be found in Section 2.3. However, the second component related to the balancing between quarks of different flavours will be reflected in the balance function yield. Thus, a higher yield found for a particular pair of hadrons, compared with yields for other pairs in the same centrality class, can be attributed to the preferential balancing between the first pair as well as the corresponding constituent quarks. The best way of probing this is by comparing the results for pairs of identical hadrons, e.g. $\pi^{+} \pi^{-}$, with the results for cross-correlations, e.g. $\pi^{+} \mathrm{K}^{-}, \pi^{+} \overline{\mathrm{p}}$ and so on. In this example, while the dominant contribution is expected for $\pi^{+} \pi^{-}$, one possibility would be that, at the same time, a higher yield will be measured for $\pi^{+} K^{-}$than for $\pi^{+} \bar{p}$. This would suggest that while most of positive pions are balanced by negative pions, at the same time, it is more probable to find a 
positive pion balanced by a negative kaon than by an antiproton. In addition, this might suggest that while light quarks ( $u$ and $d$ ) are preferentially balanced by each other, a balancing between light and strange quarks is also possible. Furthermore, the difference in meson and baryon production rates can influence the difference in yields of $\pi^{+} \pi^{-}$and $\pi^{+} \mathrm{K}^{-}$with respect to $\pi^{+} \overline{\mathrm{p}}$.

An increase in the balance function yield in central with respect to peripheral collisions might be interpreted as a consequence of the effect of radial flow. Smaller radial flow in peripheral collisions which drives the broadening of the balance function width might lead to part of the correlated pairs falling outside the detector acceptance and, thus, being lost. Another possible interpretation might be simply a larger amount of balancing charges being produced in central collisions.

Lastly, while the main contribution to the measured charge balance function is driven by the charge conservation, other additional sources, considered as background effects, such as resonance decays or Coulomb interactions, could contribute as well. Moreover, quantum-statistics correlations of identical bosons (also called HBT effects) [89] can affect the shape of the balance function for bosonic pairs at the level of like-sign correlation subtraction.

\subsubsection{Proton-nucleus and proton-proton collisions}

In the previous section it was shown that in heavy-ion collisions the presence of a QGP medium leads to the narrowing of the balance function in central with respect to peripheral collisions.

In pp or p-A collisions, a naive expectation of no QGP formation would mean that the hadronization process should be nearly instantaneous. Therefore, the discussion about early or late creation time of charges should not be relevant anymore. Taking this into account together with the expected absence of collective effects should lead to the balance function width being independent of multiplicity in small systems, contrary to the case of heavy-ion collisions. In addition, due to an earlier hadronization in small compared with large systems, an overall broader width in p-A or pp than in A-A collisions should be expected.

However, recent observations of signs of collectivity in small systems (see Section 1.3) might challenge the aforementioned assumptions. Since these collective effects develop as a function of multiplicity, one would naturally expect a decrease of the balance function width in higher with respect to lower multiplicities, similarly to large systems. Moreover, these observations not only point in the direction of a possible creation of a QGP medium in small systems, but also its more explosive expansion compared with heavy-ions case. The latter statement 
is deduced from the extraction of transverse collective flow velocity $<\beta_{\mathrm{T}}>$ from the $\mathrm{p}_{\mathrm{T}}$ spectra of identified hadrons using the blast-wave parametrization (see Section 1.2.3 for more details), that shows that $\left\langle\beta_{\mathrm{T}}\right\rangle$ values in $\mathrm{p}-\mathrm{Pb}$ and $\mathrm{pp}$ collisions reach larger values at the freeze-out surface than in $\mathrm{Pb}-\mathrm{Pb}$ collisions at similar multiplicities [90], [91]. This suggests that small systems get more "explosive" leading to a more violent and faster expansion of the medium than the A-A system. In view of such observations, the expectation of a broader width in high-multiplicity p-A or pp collisions than in heavy-ion collisions might be revisited.

\subsection{Lessons learned from models}

The sensitivity of the balance function to things like the freeze-out conditions, the collective expansion of the medium and diffusion effects has been studied with the help of several models, discussed below.

The effect of creation time of different quark flavours in the QGP evolution on the width of the balance function constructed for hadrons was demonstrated in [86]. In this paper the authors propose the so-called two-wave quark production model based on considerations of early and later stage charge creation discussed earlier in Section 2.2.1. This model connects the final separation $G_{\alpha \beta}(\eta)$ between any two hadronic species of types $\alpha$ and $\beta$ to the separation between their constituent quarks of types a and b. The separation between quarks built in the early stage, i.e. the QGP phase, is given by a $\sigma_{\mathrm{QGP}}$ parameter, while the separation in the later stage, i.e. at hadronization, is represented by a $\sigma_{\text {HAD }}$ parameter. These unknown parameters can be then extracted from the analysis of experimental $G_{\alpha \beta}(\eta)$ for identified hadrons, which allows to predict the dependence of the shape of the balance function for hadron pairs on the flavour of the constituent quarks and their corresponding creation time.

While in this model more than a half of the up and down quarks are created at the hadronization, most of $s \bar{s}$ pairs are produced in the early QGP phase. The results generated for pion and kaon pairs are shown in Figure 2.2. In the upper panel of the left plot, it can be seen that, as expected from the later production of $\mathrm{u}$ and $\mathrm{d}$ quarks, the balance function for pions is dominated by the $\sigma_{\mathrm{HAD}}$ term while the contribution from $\sigma_{\mathrm{QGP}}$ remains small. In addition, for a fixed $\sigma_{\mathrm{HAD}}$ the lower panel shows the change of the shape for different $\sigma_{\mathrm{QGP}}$ values. Oppositely, since very few strange quarks are produced close to hadronization, in the balance function for kaon pairs, shown in the upper panel of the right plot,

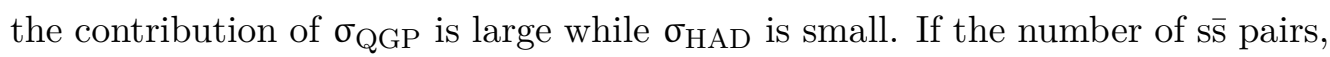


produced early, is reduced by half, the $\sigma_{\mathrm{QGP}}$ component is not dominant anymore and the hadronization contribution starts to grow, leading to a much narrower correlation shown in the lower panel of the same plot. This way, comparing predictions of this model to the data can provide an information on the creation time of different quark flavours in the QGP.
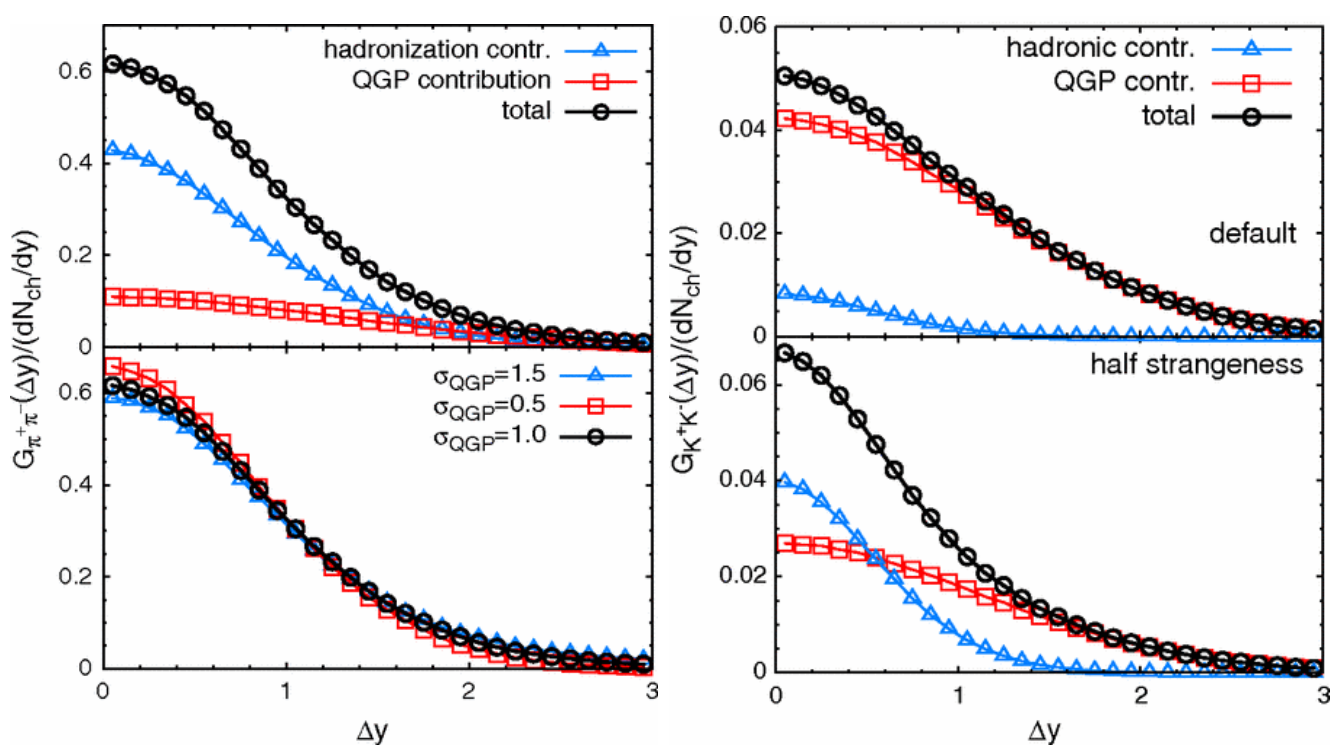

FiguRE 2.2: The balance function of pion (left) and kaon (right) pairs separated by the QGP and hadronization contributions in two-wave quark production model

In [85], the effect of the creation time of balancing partners was studied in a different way at the level of hadrons, i.e. by investigating the change in $\sigma_{\text {therm }}$ from Equation 2.5 with the variation of the freeze-out temperature. This was done using a simple Bjorken parametrization [92], where a modelled thermal source, that expands collectively with a certain velocity along the $\mathrm{z}$ axis, is used to generate hadron pairs of opposite charge at the same location of space and time. The balance functions are constructed for an expanding pion and proton gas at two different chemical freeze-out temperatures of 165 and $225 \mathrm{MeV}$ and the results are shown in Figure 2.3.

It can be seen, as expected from the $\sigma_{\text {therm }}$ term dependence on the temperature, that for both particle species the balance function width decreases when the freeze-out takes place at a lower temperature. Moreover, due to the dependence of this term on the hadron mass, the balance function of proton pairs is narrower than the one of pion pairs. In addition, protons are more sensitive to the change in temperature. This allows to conclude that balance functions measured for pairs of heavier hadrons, such as baryons or strange hadrons, can provide more detailed information on the freeze-out conditions than the ones of lighter hadrons. 
Additionally, in the same figure, balance functions generated in the Bjorken model are compared with similar results generated using the PYTHIA model [93] for minimum bias pp collisions at $\sqrt{\mathrm{s}}=200 \mathrm{GeV}$. While an expectation for a scenario with the QGP formation and its hydrodynamic evolution is often described in terms of the Bjorken model, PYTHIA, based on Lund string fragmentation model, represents the expectation for a scenario with no QGP phase. It can be seen that the balance functions in pp collisions for both particle species are much broader than the ones in the Bjorken model. The conclusion of the authors at the time when these studies were done, i.e. the time of searches for evidences of a QGP presence in the heavy-ion case and the expectation of no medium effects in smaller systems, was connected to the former understanding of physics phenomena. It stated that assuming that experimental balance functions in pp collisions would have been well described by such string dynamics, this comparison would suggest that narrower balance functions point to a delayed hadronization in the QGP, i.e. a thermal production of hadrons at a lower temperature and thus at later times in the evolution of the heavy ion reaction. This way, the narrowing of the balance function width was proposed as one of the signatures of the creation of a QGP medium in heavy-ion collisions.

Within the up-to-date understanding, the creation of a QGP and the subsequent collective phenomena that develop in heavy-ion collisions is a well-established concept confirmed by many experimental studies. However, recent observations of signs of collectivity also in high-multiplicity collisions in small systems (see Section 1.3) suggest not only the formation of a QGP medium, but also its more explosive expansion in case of $\mathrm{p}-\mathrm{Pb}$ or $\mathrm{pp}$ compared with $\mathrm{Pb}-\mathrm{Pb}$ collisions (see Section 2.2.2 for more details). Therefore, the aforementioned conclusion connected to an expectation of a narrower balance function width in heavy-ion collisions than in pp collisions remains valid for a minimum bias sample, however it might not be valid anymore if also high-multiplicity events in pp collisions are considered. 


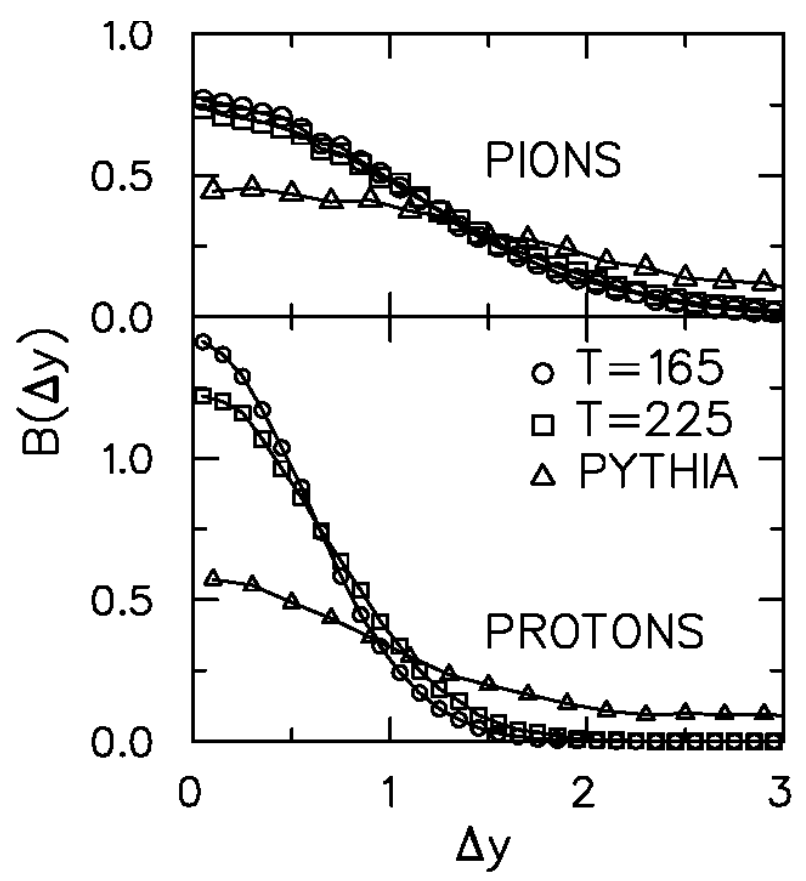

FiguRE 2.3: Balance functions of pion and proton pairs in the relative rapidity as predicted in a Bjorken thermal model for two different freeze-out temperatures of $225 \mathrm{MeV}$ and $165 \mathrm{MeV}$. The prediction from PYTHIA model for minimum bias pp collisions at $\sqrt{\mathrm{s}}=200 \mathrm{GeV}$ is also shown

In the same paper, to illustrate the effect of separation of the balancing charges due to rescattering, i.e. reflected by the $\sigma_{\text {flow }}$ term of Equation 2.5, this part is modelled on top of the initial configuration of the same model. The balance function is calculated for two scenarios: in the first one the charges are produced early, i.e. at $\tau=1 \mathrm{fm} / \mathrm{c}$ when the temperature is $225 \mathrm{MeV}$, and later at $\tau=$ $9 \mathrm{fm} / \mathrm{c}$ and temperature of $165 \mathrm{MeV}$. The resulting balance function width in rapidity as a function of number of collisions $\mathrm{N}_{\text {coll }}$ is shown in Figure 2.4 for pions, kaons and protons. It can be seen that for an early stage creation scenario the width becomes significantly broader with increasing amount of collisions until a certain point. After that, since the collision numbers reach large values, the charge does not diffuse anymore and the balance function width decreases due to the cooling. For the particles created later the width is not affected that much by rescattering and the cooling drives its narrowing over the time. These results suggest that the observation of a broader width might be a signal of an early-stage production of quarks for all particle species. 


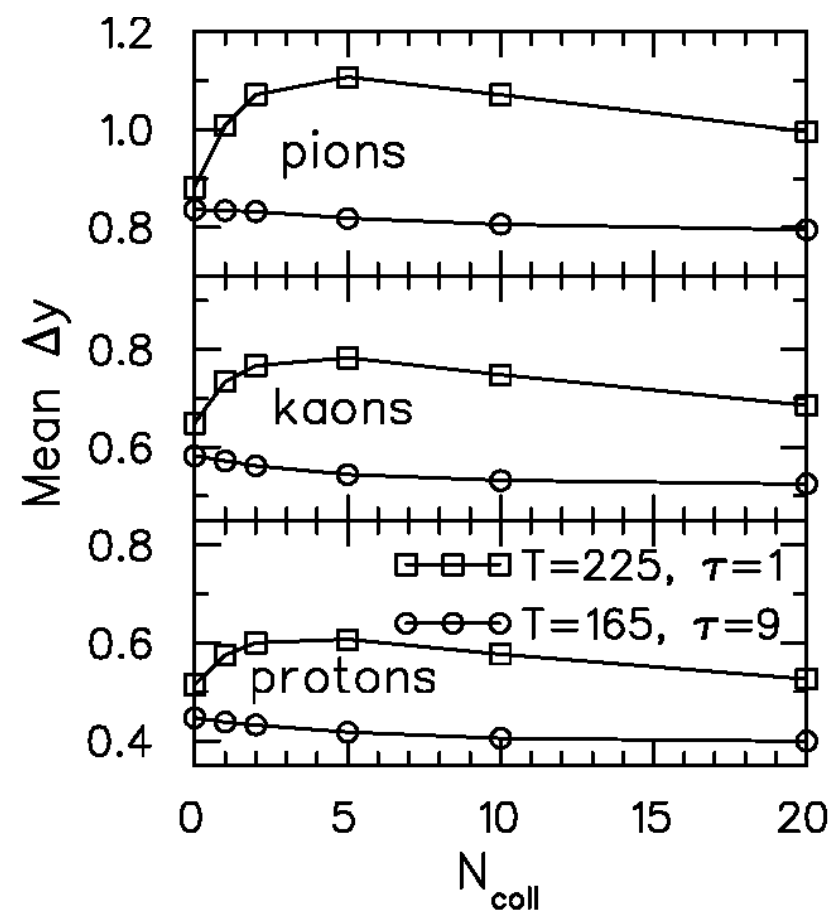

FiguRE 2.4: The width of the balance function of pion, kaon and proton pairs in the relative rapidity as predicted in a simple Bjorken thermal model for early and late creation time as a function of number of collisions

In [94], the effect of diffusion was studied at the level of quark pairs. The authors propose a method to estimate the diffusivity of light quarks $(\mathrm{u}, \mathrm{d}, \mathrm{s})$, which is not well understood yet. This is based on a comparison of predictions from the hybrid model [95] with experimentally measured balance functions of identified hadrons. In this model, a detailed simulation of the production and diffusion of balancing quarks is superimposed onto a description of the QGP stage, using relativistic viscous hydrodynamics, coupled to a hadronic rescattering phase. Once the quarks of type $a$ and $b$ are created, they diffuse away from one another according to the value of the diffusion coefficient. The quark-quark correlations in the QGP are evolved according to four different choices for the diffusivity, a default value being the one reported in lattice calculations, $\mathrm{D}_{\text {latt }}$, and the others being half, double and four times the lattice diffusivity. Figure 2.5 shows the resulting balance functions in the relative azimuthal angle for all charged particles, kaon and proton pairs. It can be seen that the distributions turn out to be broader for the larger values of $\mathrm{D}_{\text {latt }}$ for all considered particle species. However, the balance function for charged particles seems to be the least sensitive to the choice of $\mathrm{D}_{\text {latt }}$ parameter which is explained by the fact that it is dominated by later-stage production of charge associated with hadronization in this model. On the contrary, the balance function for kaon and proton pairs is affected more due to an early production of strange quarks and baryons. The comparison of 
these results with the measured balance functions can thus put robust constrains on the diffusivity of all three light quark flavours.

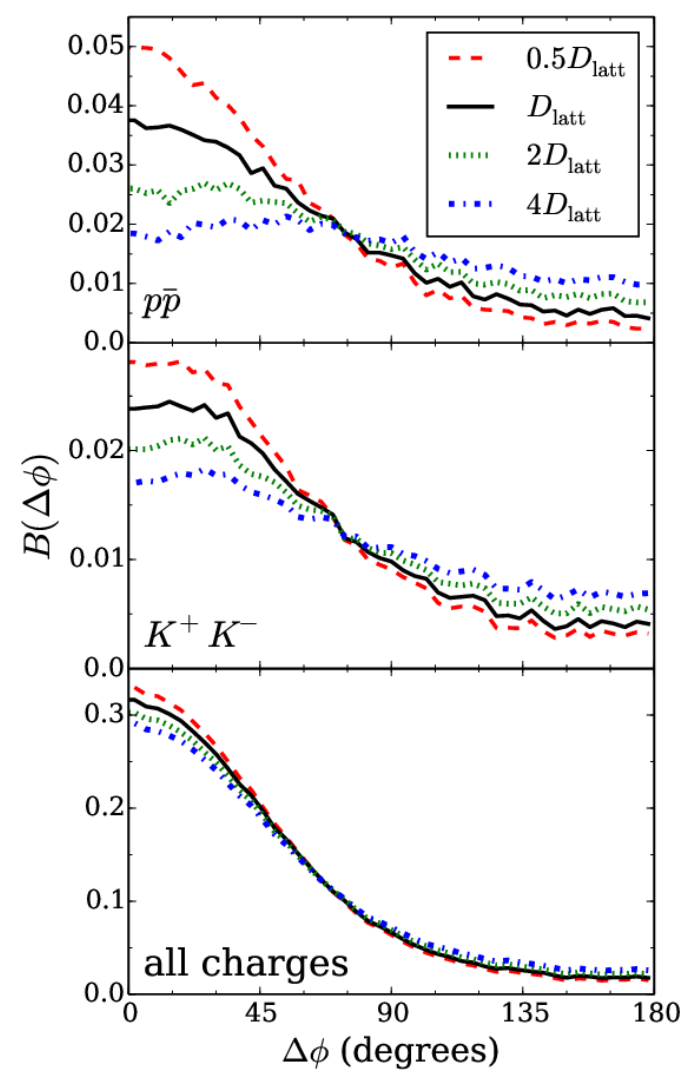

Figure 2.5: Balance functions of charged particles, kaon and proton pairs in the relative azimuthal angle for four different choices of the diffusivity of light quarks

The charge balance functions in the relative azimuthal angle were also investigated in [96], where they were shown to be a very sensitive probe of the freeze-out temperature and transverse velocity. This can be demonstrated by comparing the results for nonresonant pions from the single freeze-out thermal model [97] with the prediction from blast-wave parameterization [98]. In the first model the kinetic freeze-out happens at the same time as the chemical one at a fixed temperature of $165 \mathrm{MeV}$ and an average transverse velocity $\left\langle\beta_{\mathrm{T}}>\right.$ of 0.5 . Figure 2.6 shows the balance function for non-resonant pions (dashed red line) given by the emission of $\pi^{+} \pi^{-}$from a local thermal source obtained in this model. In the blast-wave parameterization the kinetic freeze-out happens at some moment after the chemical freeze-out. Therefore, the emission happens at a smaller freeze-out temperature of $90 \mathrm{MeV}$ but at the same time at more significant $<\beta_{\mathrm{T}}>$ of 0.6 . The balance function of the non-resonant pions in the blast-wave model (black solid line) is much narrower than in the first model. This is due to fact that the 
emission at smaller initial temperature and larger transverse flow produces pairs more focused in the azimuthal angle.

Finally, in the same paper the contribution of resonances was studied. As was already mentioned in Section 2.2.1, the decays of neutral hadronic resonances can affect the shape of the final balance function distribution. To illustrate the effect of resonances for pion pairs, Figure 2.6 shows the balance function for $\pi^{+} \pi^{-}$pairs originating from $\rho$ resonance decays (dashed black line) obtained in the single freeze-out thermal model. It can be seen that given the conditions used in this model $30 \%$ of pion pairs originate from the decay of resonances. The balance function is also much broader for resonant pions due to additional back-to-back decay contribution.

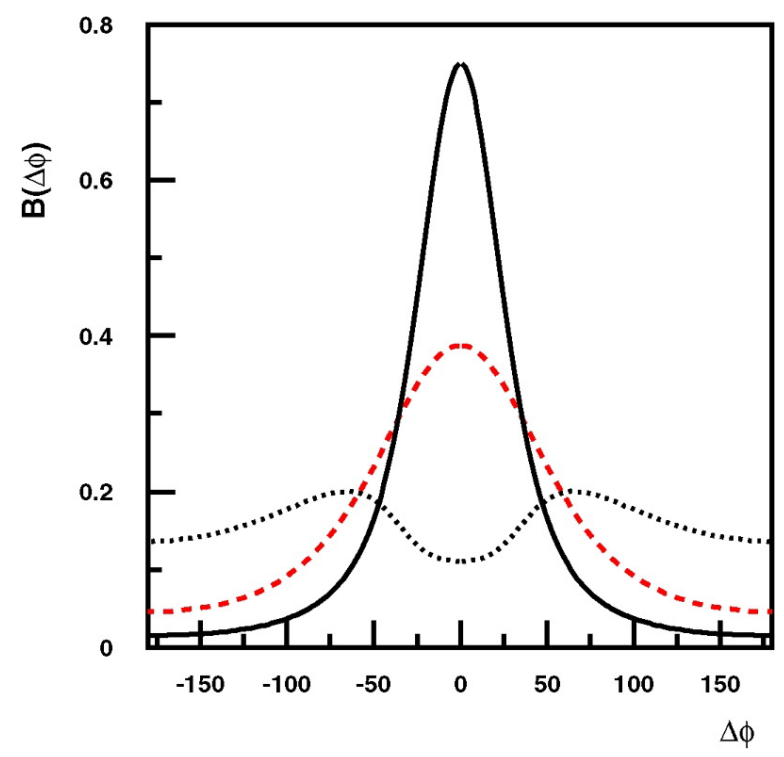

FIGURE 2.6: Balance function of pion pairs in the relative azimuthal angle in thermal models calculated for two different freeze-out conditions: $\mathrm{T}_{\mathrm{f}}=165$ $\mathrm{MeV},<\beta_{\mathrm{T}}>=0.5$ (dashed line for nonresonant pions and dotted line for pions from the decay of a $\rho$ resonance) and $\mathrm{T}_{\mathrm{f}}=90 \mathrm{MeV},\left\langle\beta_{\mathrm{T}}>=0.6\right.$ (solid line for nonresonant pions)

\subsection{Where we stand experimentally}

With the previous model expectations in mind, this section presents the results of previous experimental balance function measurements.

The balance function definition is derived from the associated charge density balance that was developed to measure the charge-dependent correlations in nondiffractive inelastic events [99]. Later on, similar method was used to investigate the jet fragmentation properties in pp collisions at the ISR [100] as well as 
to study strangeness correlations [101] and baryon correlations [102] in $\mathrm{e}^{+} \mathrm{e}^{-}$ collisions at $\sqrt{\mathrm{s}}=29 \mathrm{GeV}$ at PETRA.

The first measurement of the balance function in relative pseudorapidity for charged particles and in relative rapidity for identified pion pairs was reported by the STAR experiment in Au-Au collisions at $\sqrt{\mathrm{S}_{\mathrm{NN}}}=130 \mathrm{GeV}$ [103]. Only primary charged particles were analyzed in the transverse momentum range of $0.1<\mathrm{p}_{\mathrm{T}}<2 \mathrm{GeV} / \mathrm{c}$. The upper panel of Figure 2.7 presents the resulting distributions for central and peripheral collisions for charged particles on the left, while similar results for pions are shown on the right. To guide the eye, Gaussian fits are also plotted. In both cases, the balance function distribution is narrower in central than in peripheral collisions. In order to better quantify the effect of the narrowing, the corresponding balance function widths for four centrality classes are shown in Figure 2.8 as a function of the impact parameter fraction $\mathrm{b} / \mathrm{b}_{\max }$ with the points of peripheral (central) collisions positioned on the right (left) side of the x-axis. The results for charged particles are presented on the left, while for pions on the right. It can be seen that for both charged particles and pions the width of the balance function in central collisions is significantly narrower than the one in peripheral collisions. At that time, such observation was stated to be qualitatively consistent with theoretical expectations of the delayed hadronization in central collisions described in Section 2.2.1. However, a need for a more detailed study of the balance function width sensitivity to collective flow effects as well as other effects that could lead to the narrowing, such as resonance decays, was highlighted.

In addition to data points, similar results obtained using the event mixing technique and shuffling procedure are plotted for charged particles in Figures 2.7 and 2.8. In the event mixing technique, the balance function is constructed by randomly choosing particles from different (mixed) events with similar vertex position and multiplicity. Since this way all the correlations caused by global charge conservation are removed, the balance function from mixed events is at zero in all centrality bins. The shuffling procedure is a different mixing method where the pseudorapidities of all charged particles within each event are randomly shuffled while keeping the electric charge of each track the same. The shuffled events thus include all the momentum correlations and the total charge observed in the original event, but the charge-momentum correlations are removed. This procedure is therefore used in order to estimate the maximum possible width of the balance function when the dynamical particle correlations are removed but the correlations from global charge conservation are preserved. In both figures, it can be seen that the results for shuffled data are broader than the one from the real data in each centrality class. 
In the lower panel of Figure 2.7, the balance functions extracted from HIJING events [104] simulated in the STAR detector geometry using GEANT [105] are shown. The HIJING generator is based on the excitation of strings and their subsequent hadronization according to the LUND model [106]. The Au-Au collisions are treated as a superposition of independent nucleon-nucleon scatterings, that corresponds to an expectation for a collision with no QGP formation. The results for pion pairs are similar to those for charged particles except for a dip near $\Delta y=0$ explained as the combined effect of Bose-Einstein correlations and Coulomb interactions [85]. In HIJING results this dip is not present since these effects are not included in the simulation. Moreover, while a clear dependence on centrality is seen in the data, the balance function for HIJING events is plotted for integrated centrality since no centrality dependence was observed. In order to demonstrate the latter, the corresponding balance function widths as a function of $\mathrm{b} / \mathrm{b}_{\max }$ are compared to the experimental results in Figure 2.8. It is observed that the narrowing of the width with increasing centrality in the data is not reproduced by the HIJING simulation, which is expected due to the absence of medium effects in HIJING.
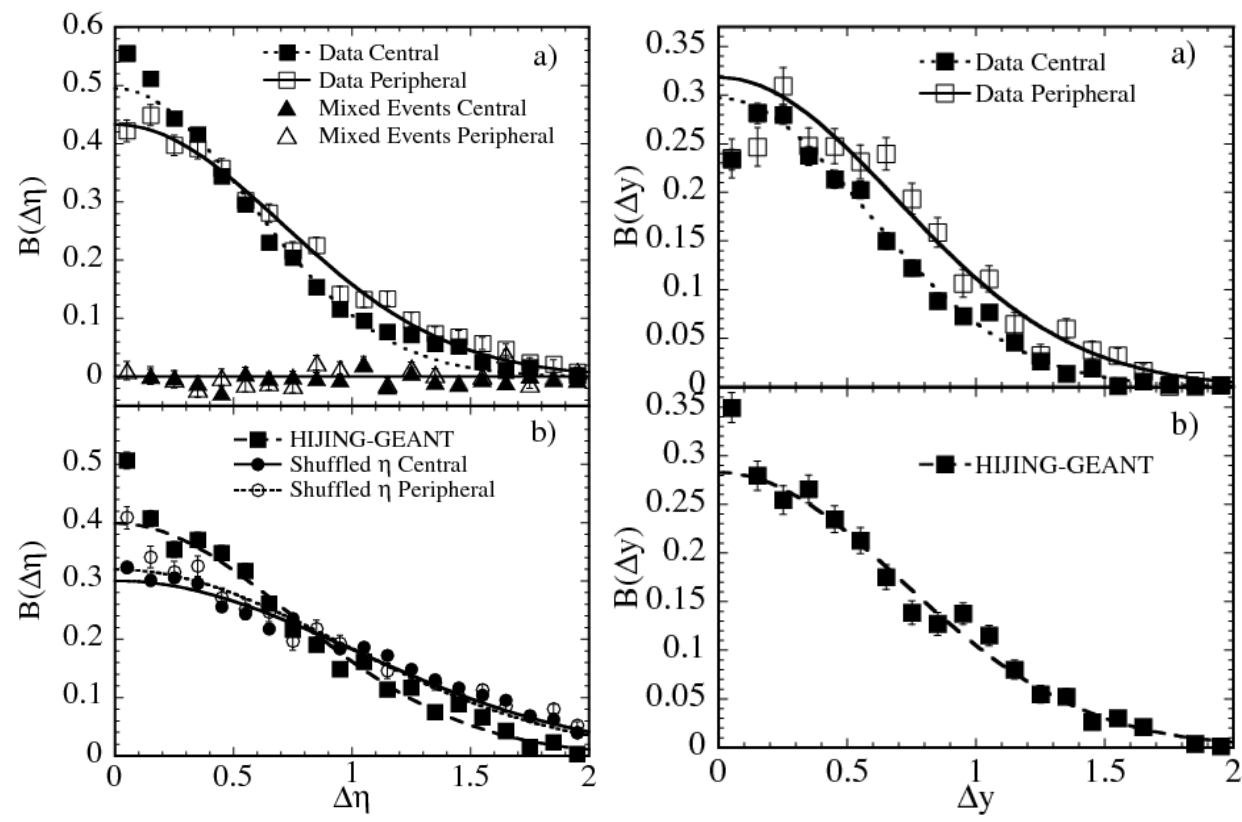

FIGURE 2.7: The balance function in relative pseudorapidity for charged particles (left) and in relative rapidity for pions (right) in $\mathrm{Au}-\mathrm{Au}$ collisions at $\sqrt{\mathrm{S}_{\mathrm{NN}}}=130 \mathrm{GeV}$. The results from mixing technique, shuffling procedure and predictions from HIJING for charged particles are also shown 

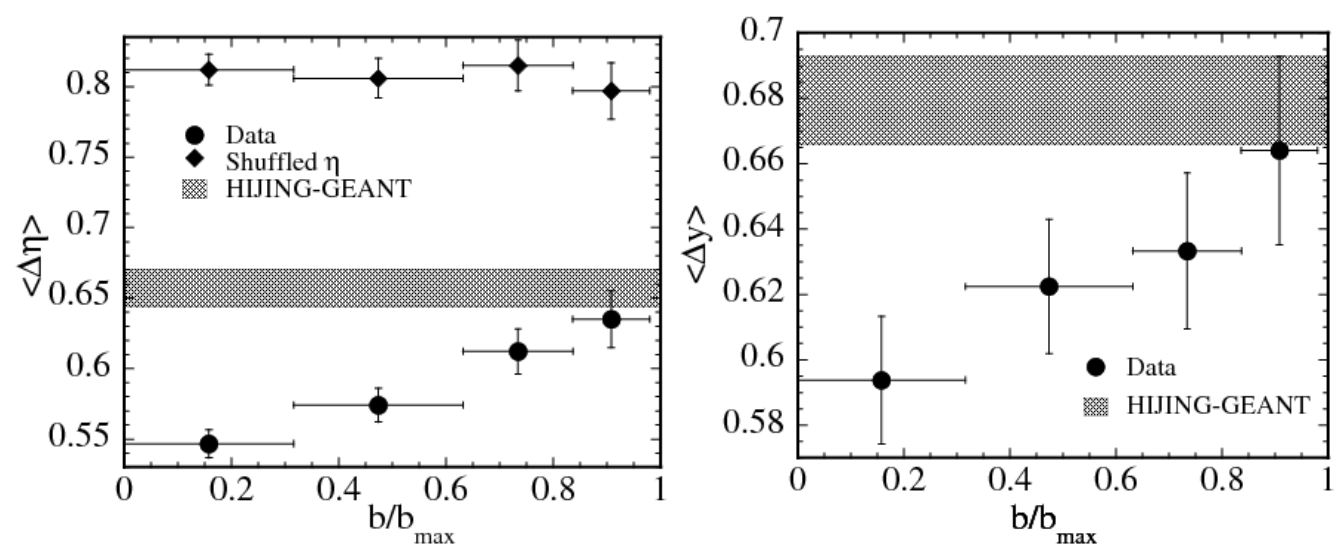

FIgURE 2.8: The balance function width $\langle\Delta \eta\rangle$ for charged particles (left) and $\langle\Delta y\rangle$ for pions (right) as a function of normalized impact parameter $\left(b / b_{\max }\right)$ in $\mathrm{Au}-\mathrm{Au}$ collisions at $\sqrt{\mathrm{S}_{\mathrm{NN}}}=130 \mathrm{GeV}$. The results of the shuffling procedure and the HIJING prediction for charged particles are also shown

Later on, similar centrality-dependent measurement was performed by the NA49 collaboration in $\mathrm{Pb}-\mathrm{Pb}$ collisions at the energy of $\sqrt{\mathrm{s}_{\mathrm{NN}}}=17.2 \mathrm{GeV}$ [107]. The results of the balance function in relative pseudorapidity are shown for three centrality bins (Veto in the plot) from the most central (Veto 1) to the most peripheral (Veto 6) in Figure 2.9 on the left. It can be seen that the distribution is narrower in the most central collisions in comparison to the one in most peripheral ones, which is in qualitative agreement with previous STAR results for $\mathrm{Au}-\mathrm{Au}$ data. To illustrate the agreement better, the balance function width in both cases is plotted on the right as a function of $b / b_{\max }$. Since an observed decrease was found to be of a similar order at both energies, the authors concluded that the relative narrowing of the width was independent of energy. However, a systematic decrease seen in the absolute value at the higher STAR energy triggered an energy-dependent scan of the width at other SPS energies of 12.3, 8.8, 7.6 and $6.3 \mathrm{GeV}$ [108]. This study confirmed that the width became continuously narrower at higher energies. 

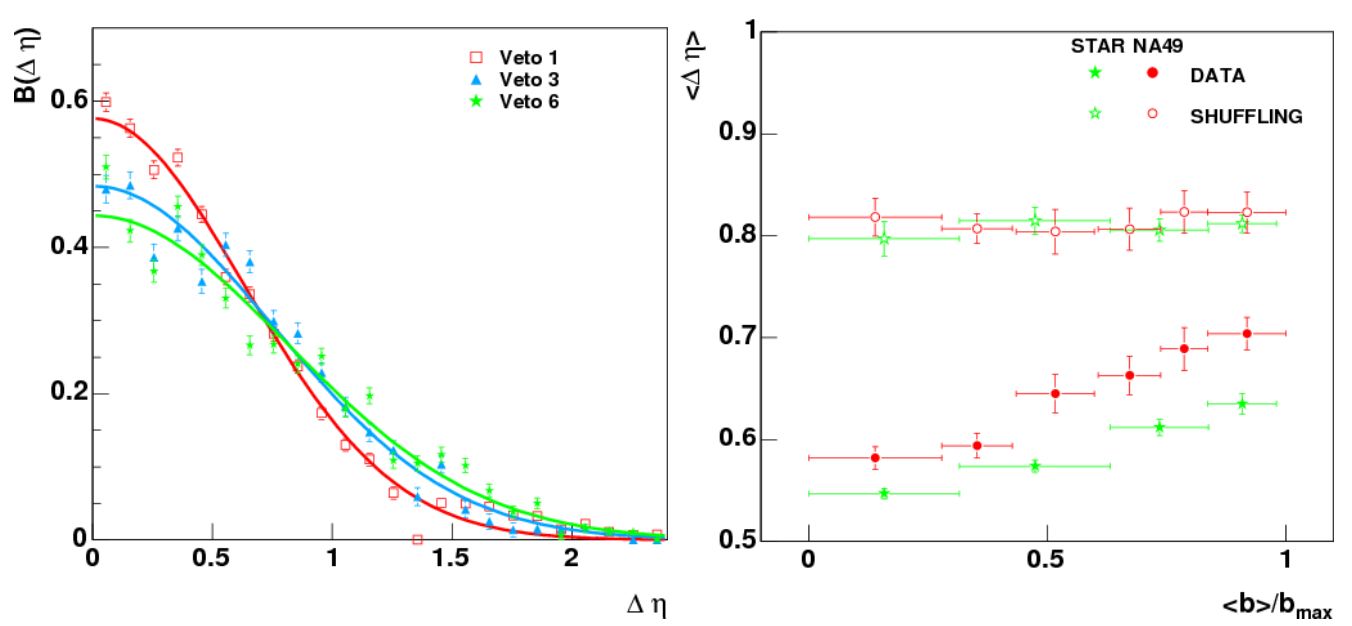

FIgURE 2.9: The balance function in relative pseudorapidity for charged particles as a function of centrality (left). The balance function width $\langle\Delta \eta\rangle$ as a function of normalized impact parameter $\left(\langle\mathrm{b}\rangle / \mathrm{b}_{\max }\right)$ in $\mathrm{Pb}-\mathrm{Pb}$ collisions at $\sqrt{\mathrm{s}_{\mathrm{NN}}}=17.2 \mathrm{GeV}$ compared to result in $\mathrm{Au}-\mathrm{Au}$ collisions at $\sqrt{\mathrm{S}_{\mathrm{NN}}}=130 \mathrm{GeV}$

(right)

To interpret the narrowing of the width in Figure 2.9, the results were compared to several microscopic models in addition to HIJING, such as UrQMD [109] and AMPT [78]. In UrQMD, the main difference with respect to HIJING was the inclusion of the hadronic rescattering phase. The main difference of the AMPT model with respect to HIJING and UrQMD was that it incorporated the time evolution of partons before their hadronization. The resulting balance function widths are shown in Figure 2.10 [108] as a function of the mean number of wounded nucleons $\left\langle\mathrm{N}_{\mathrm{w}}\right\rangle$ (similar to $\left\langle\mathrm{N}_{\text {part }}\right\rangle$, see Section 1.2.2), where higher $\left\langle\mathrm{N}_{\mathrm{w}}\right\rangle$ corresponds to more central collisions. Since HIJING and UrQMD failed to reproduce the centrality dependence in the data, while AMPT predicted a similar centrality dependence, the authors stated that this was an indication of the balance function sensitivity to the presence of a partonic stage and the subsequent time evolution of particle production. 


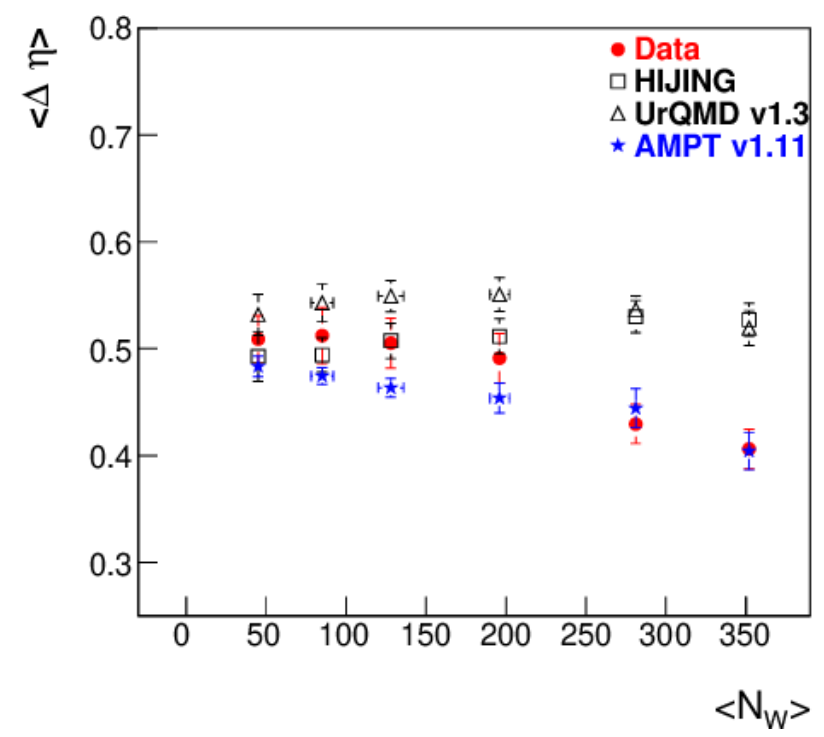

FiguRE 2.10: The balance function width $\langle\Delta \eta\rangle$ for charged particles as a function of the mean number of wounded nucleons $\left\langle\mathrm{N}_{\mathrm{w}}\right\rangle$ in $\mathrm{Pb}-\mathrm{Pb}$ collisions at $\sqrt{\mathrm{s}_{\mathrm{NN}}}=17.2 \mathrm{GeV}$ compared to model predictions

The first balance function measurement at the LHC energies was reported by ALICE in [110] for charged particles with $0.3<\mathrm{p}_{\mathrm{T}}<1.5 \mathrm{GeV} / \mathrm{c}$ in $\mathrm{Pb}-\mathrm{Pb}$ collisions at $\sqrt{\mathrm{S}_{\mathrm{NN}}}=2.76 \mathrm{TeV}$. A comparison of the resulting balance function width in $\Delta \eta$ and $\Delta \varphi$ to STAR results in Au-Au collisions at $\sqrt{\mathrm{s}_{\mathrm{NN}}}=200 \mathrm{GeV}$ is shown in Figure 2.11 on the left. The observation is similar to the one seen earlier in the comparison between the results from STAR and NA49 shown on the right in Figure 2.9: the relative narrowing for more central events is the same at both energies, but the absolute values of the widths are significantly narrower at the higher LHC energies. The authors stated that this is consistent with the idea of an effect of larger radial flow present at the LHC with respect to RHIC combined with a longer-lived QGP phase with the consequence of a smaller separation between charge pairs when created at hadronization.

The results were compared with several models as demonstrated in Figure 2.11 on the right. It can be seen that the results from HIJING do not show any centrality dependence and in $\Delta \varphi$ the resulting width is much broader than in the data. The latter is consistent with the absence of radial flow effects in the model. In addition, different configurations of AMPT model were used: default, string melting and string melting without hadronic rescattering. The main differences between the two first configurations is the extended description of a partonic stage in the string melting tune and the mechanism of combining partons into hadrons: in the first case the Lund string fragmentation model is used, while in the second the quark coalescence model is used. In both configurations the hadronic rescattering phase is also included. In the third configuration, this 
hadronic rescattering phase was switched off. The results for the default and string melting tunes were observed to be quite close to each other in both dimensions. In $\Delta \eta$ both tunes fail to reproduce the narrowing, but in $\Delta \varphi$ both of them qualitatively reproduce the trend. However, quantitatively the results for the default tune reproduce the narrowing in $\Delta \varphi$ better. This is due to the higher value of radial flow in the default configuration with respect to the string melting one. When the hadronic rescattering phase is switched off in the third tune, the balance function width in $\Delta \varphi$ becomes significantly broader. This is caused by an exclusion of a large part of radial flow building up in this phase which enhances the correlation.
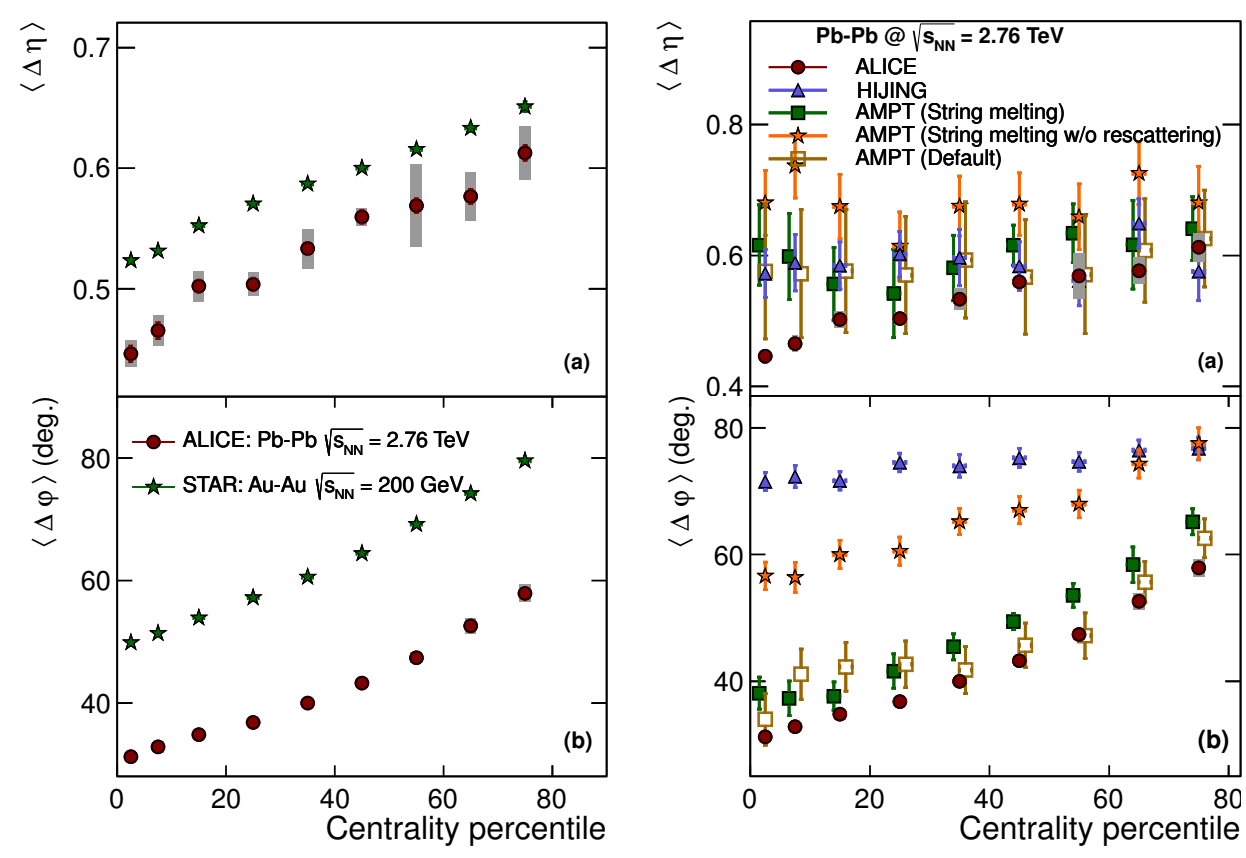

FiguRE 2.11: The balance function width $\langle\Delta \eta\rangle$ and $\langle\Delta \varphi\rangle$ for charged particles in $\mathrm{Pb}-\mathrm{Pb}$ collisions at $\sqrt{\mathrm{S}_{\mathrm{NN}}}=2.76 \mathrm{TeV}$ compared to the same measurement in $\mathrm{Au}-\mathrm{Au}$ collisions at $\sqrt{\mathrm{S}_{\mathrm{NN}}}=200 \mathrm{GeV}$ (left) and model predictions (right)

In the second, most recent measurement by ALICE reported in [111], the previous studies were extended to $\mathrm{p}-\mathrm{Pb}$ and pp collisions at $\sqrt{\mathrm{s}_{\mathrm{NN}}}=7 \mathrm{TeV}$ and $\sqrt{\mathrm{s}}=$ $5.02 \mathrm{TeV}$, respectively, as well as for higher transverse momenta ranges. The comparison of the balance function width as a function of multiplicity for all three systems is shown in Figure 2.12. In addition, the results at intermediate and high $\mathrm{p}_{\mathrm{T}}$ ranges are presented. It was observed for the first time that the narrowing of the balance function is the feature only of the low $\mathrm{p}_{\mathrm{T}}$ range, while the results are independent of multiplicity for the intermediate and higher $\mathrm{p}_{\mathrm{T}}$ ranges in all systems. This is consistent with an idea of initial hard parton scattering and subsequent fragmentation being the main origin of charge correlations in these ranges. 
For $\mathrm{p}-\mathrm{Pb}$ and $\mathrm{pp}$ the balance function width has similar values at similar multiplicities which might point to a common physics mechanism responsible for this narrowing across systems. However, the values in $\mathrm{p}-\mathrm{Pb}$ and $\mathrm{Pb}-\mathrm{Pb}$ are significantly different at similar multiplicities in $\Delta \eta$ and are a bit closer in $\Delta \varphi$. The authors stated that this difference could be caused by a different mechanism driving the narrowing in $\mathrm{Pb}-\mathrm{Pb}$ with respect to small systems.
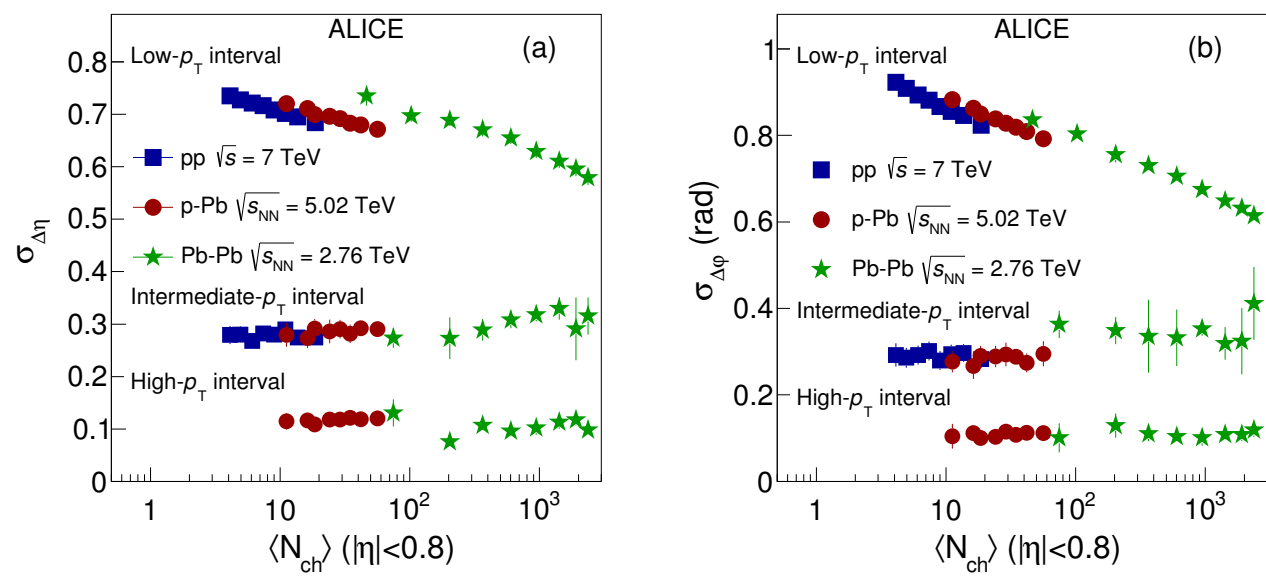

Figure 2.12: The balance function width $\sigma(\Delta \eta)$ (left) and $\sigma(\Delta \varphi)$ (right) for charged particles in $\mathrm{Pb}-\mathrm{Pb}, \mathrm{p}-\mathrm{Pb}$ and pp collisions at $\sqrt{\mathrm{s}_{\mathrm{NN}}}=2.76 \mathrm{TeV}$, $\sqrt{\mathrm{s}_{\mathrm{NN}}}=5.02 \mathrm{TeV}$ and $\sqrt{\mathrm{s}}=7 \mathrm{TeV}$, respectively, as a function of multiplicity in different $\mathrm{p}_{\mathrm{T}}$ intervals

To investigate this further, the results for pp collisions were compared with expectations from the PYTHIA8 model [112] with and without color reconnection. Color reconnection is one of the alternative microscopic mechanisms proposed for pp collisions that might lead to signs of collectivity in small systems (see Section 1.3) [83], [113]. It resembles the effect of radial flow with multiplicity at the microscopic level, i.e. leads to an increased transverse boost for higher multiplicities that results in enhancement of correlation. This mechanism is implemented in PYTHIA8 with more details included in Section 5.2.5. The results of comparison can be seen in Figure 2.13. While the tune without color reconnection, represented by the solid line, fails completely in describing the narrowing of the balance function with increasing multiplicity, the tune with color reconnection qualitatively reproduces the narrowing in both dimensions. 

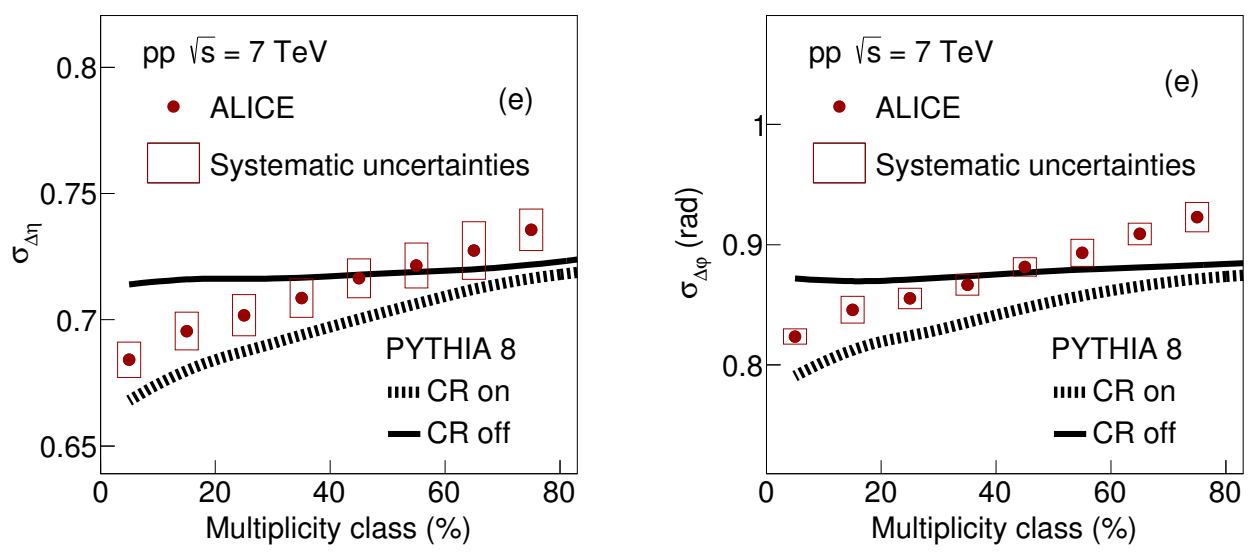

Figure 2.13: The balance function width $\sigma(\Delta \eta)$ (left) and $\sigma(\Delta \varphi)$ (right) for charged particles in pp collisions at $\sqrt{\mathrm{s}}=7 \mathrm{TeV}$ compared with predictions from PYTHIA8 model with and without color reconnection

In summary, at the level of the balance function measurement for charged particles, the results in pp collisions show qualitatively similar narrowing with increasing multiplicity as observed in heavy-ion collisions. Since for pp collisions the creation time of charges should not contribute to the narrowing due to the small size of the system (see Section 2.2.2), and in view of the latest observations of collective effects in other observables discussed previously in Chapter 1, such narrowing might be driven solely by stronger collective-like effects at higher multiplicity. The analysis of identified hadrons in pp collisions presented in this thesis provides a possibility to understand the origin behind such narrowing. 


\section{Chapter 3}

\section{ALICE Experiment}

\subsection{The Large Hadron Collider}

The Large Hadron Collider (LHC) is the most powerful particle accelerator of protons and heavy ions in the world based at CERN (Conseil Européen pour la Recherche Nucléaire) close to Geneva. In a 27-kilometer ring, shown schematically in Figure 3.1 [114], LHC hosts four main high-energy experiments at four intersection points where the circulating particle beams cross. These experiments are dedicated to the studies of different aspects of particle physics. ATLAS (A Toroidal LHC Apparatus) and CMS (Compact Muon Solenoid) are generalpurpose experiments designed to test the predictions of the Standard Model and search for new physics, while ALICE (A Large Ion-Collider Experiment) focuses on heavy-ion collisions and $\mathrm{LHCb}$ ( $\mathrm{LHC}$ b-hadron experiment) investigates the asymmetry between matter and antimatter by studying the decays of hadrons containing the bottom quark. 


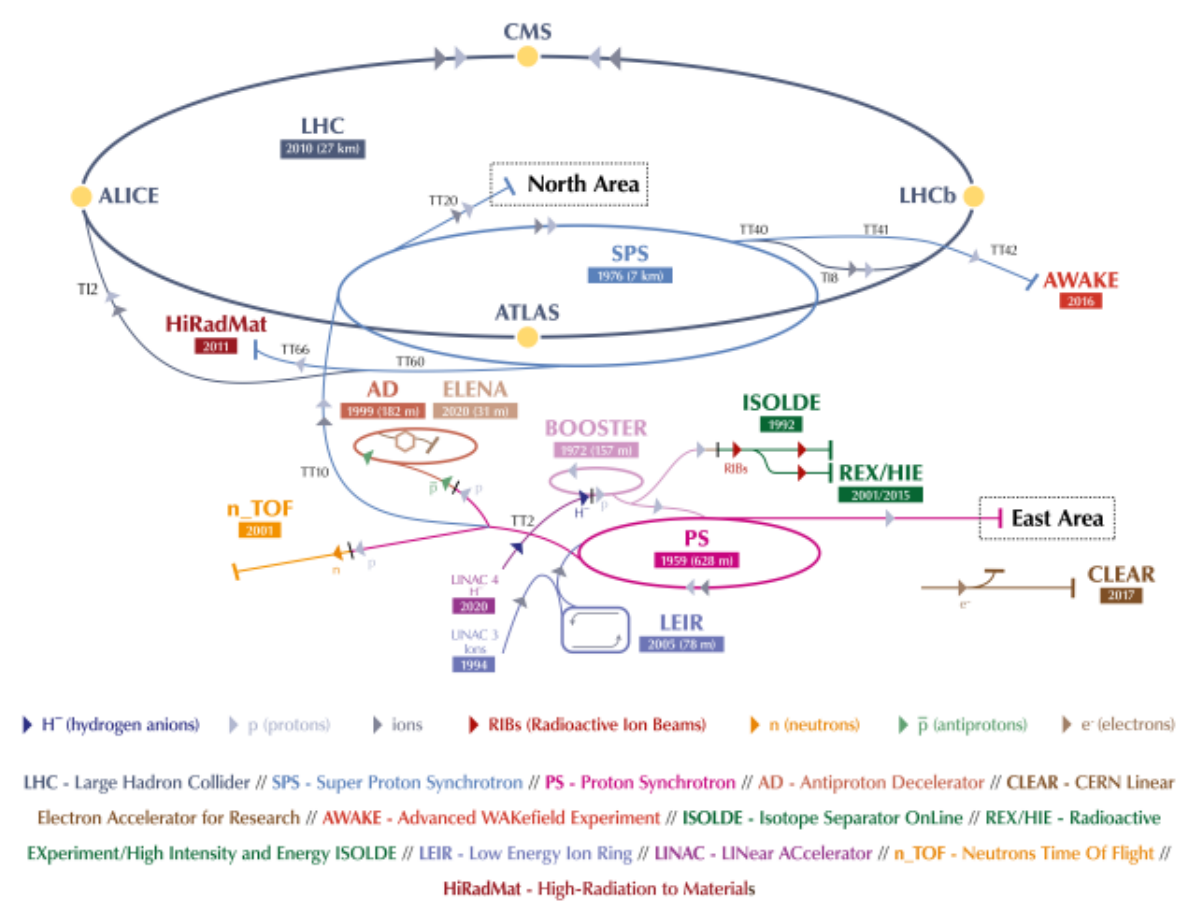

FIGURE 3.1: LHC complex

The key values that quantify the performance of particle colliders are the beam energy and the luminosity representing the number of collisions that can be produced in a detector per area per second. At the LHC, proton beams can reach the maximum energy of $6.5 \mathrm{TeV}$, while the highest energy for heavy ions is of $5.02 \mathrm{TeV}$ per nucleon pair. As for the luminosity, from 2015 till October 2018 the LHC was able to run with a peak luminosity of about $2 \times 10^{34} \mathrm{~cm}^{-2} \mathrm{~s}^{-1}$ which is two times higher than the LHC design luminosity $\left(1 \times 10^{34} \mathrm{~cm}^{-2} \mathrm{~s}^{-1}\right)$. The ongoing major upgrade will allow to increase the luminosity by a factor of five and explore the accelerator's full capacity in the nearest future.

\subsection{ALICE experiment}

The main goal of the ALICE experiment is to investigate the phase diagram of quantum chromodynamics at high energy density and temperature. This can be achieved by studying the properties of strongly interacting matter created in ultra-relativistic heavy-ion collisions called the quark-gluon plasma, described in detail in Chapter 1. The heavy-ion physics program of ALICE is complemented by the studies of proton-nucleus, proton-proton and lighter ions collisions that allow distinguishing the effects caused by the QGP formation from the ones usually associated to cold nuclear matter [115]. 
The ALICE detector consists of 18 subsystems each with specific design and technology that provide high-momentum resolution and excellent Particle Identification (PID) capabilities over a broad momentum range. This section includes a brief description of all subsystems and a slightly more detailed part about the detectors that were used for this analysis. Few detectors that are located inside a magnet with a maximum possible field of $0.5 \mathrm{~T}$ form the so called central barrel part while others belong to the forward part of the experiment.

The three main central-barrel detectors are the Inner Tracking System (ITS), the Time Projection Chamber (TPC) and the Time Of Flight (TOF). While both the ITS and the TPC provide the primary and secondary vertices reconstruction, the information on the specific energy loss $(\mathrm{dE} / \mathrm{dx})$ and momenta of the tracks, the TOF measures the track velocity and thus provides the particle's mass. The rest of the central-barrel part includes the Transition Radiation Detector (TRD) detector, two calorimeters, namely the Photon Spectrometer (PHOS) and the Electromagnetic Calorimeter (EMCal), and the High Momentum Particle Identification Detector (HMPID). The TRD together with the ITS and the TPC is used mainly for electron identification via transition radiation which allows for better electron/pion separation. The PHOS and the EMCal allow studying the thermal properties of the QGP by direct photon and jet energy loss measurements while the HMPID contributes to the identification of particles beyond the momentum range accessible by the TOF.

The forward part of the ALICE detector consists of the Photon Multiplicity Detector (PMD), the Forward Multiplicity Detector (FMD), the Zero Degree Calorimeter (ZDC), the T0 and V0 detectors. While the PMD measures eventby-event photon multiplicity, the FMD measures charged particle multiplicity in the forward acceptance region $(-3.4<\eta<-1.7$ and $1.7<\eta<5.0)$. The ZDC measures the energy of spectator nucleons at zero degree with respect to the beam axis in the pseudo-rapidity range of $4.8<\eta<5.7$. The main function of the T0 is to provide the start time for the TOF. Finally, the V0 is the main centrality estimator of ALICE and has several other functions that are described later in Section 3.2.4.

\subsubsection{ITS}

The ITS is a cylindrical tracker consisting of six layers of silicon detectors surrounding the beam pipe and covering the pseudo-rapidity range $|\eta|<0.9$ as well as full azimuth (with the exception of the dead channels). The beam pipe is a $800 \mu \mathrm{m}$-thick beryllium cylinder of $6 \mathrm{~cm}$ outer diameter, coaxial with the ITS 
detector layers. The two innermost layers are equipped with Silicon Pixel Detectors (SPD), the two intermediate layers - with Silicon Drift Detectors (SDD), while the two outermost layers consist of Silicon Strip Detectors (SSD) as shown in Figure 3.2 [116]. Such positioning and number of layers allows for better track finding and is optimized to achieve a high precision in the measurement of the distance of closest approach (DCA) of a charged particle track to the primary vertex. For instance, high particle density of $\mathrm{Pb}-\mathrm{Pb}$ collisions drives the choice for the two innermost four layers that need to be truly two-dimensional devices [117].

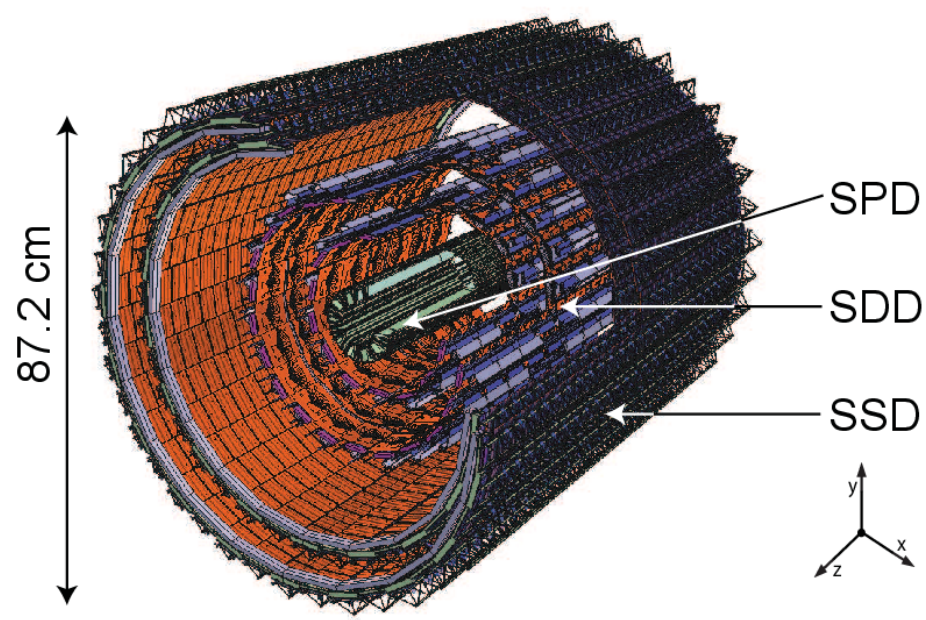

FiguRE 3.2: Layout of the ITS

One of the main goals of the ITS is to identify the secondary vertices from the decay of hyperons and heavy flavoured hadrons for the measurement of strange, charm and beauty hadron production in $\mathrm{Pb}-\mathrm{Pb}$ collisions. This is achievable due to the primary vertex reconstruction resolution being better than $100 \mu \mathrm{m}$. Besides this, the ITS improves the position, angle, and momentum resolution for TPC track reconstruction and helps recovering particles that are missed by the TPC due to the limited acceptance (very low momentum particles not reaching the TPC and very high momentum ones propagating along the $10 \%$ inactive area between adjacent TPC chambers).

The SDD and SSD have analogue readout to measure the deposited charge and therefore can be used for particle identification via the $\mathrm{dE} / \mathrm{dx}$ measurement in the non-relativistic region down to the lowest momentum at which tracks can still be reconstructed and up to around $1 \mathrm{GeV} / \mathrm{c}$. For example, pions reconstructed in the ITS standalone mode can be identified down to around $100 \mathrm{MeV} / \mathrm{c}$ with the relative momentum resolution being better than $2 \%$. For momenta larger than $3 \mathrm{GeV} / \mathrm{c}$ high spatial precision of the ITS becomes an essential element of the provided momentum resolution [117]. The $\mathrm{dE} / \mathrm{dx}$ measurement for each track is 
done using a truncated mean to minimize the influence of Landau fluctuations: the average of the lowest two points is considered if the signal is measured in all four layers, or a weighted average in case three layers are used.

The current ITS has few limitations that will be addressed in the ongoing ALICE detector upgrade that includes the replacement of the six existing ITS layers by seven concentric layers of pixel detectors using Monolithic Active Pixel Sensors (MAPS) [118]. The upgraded detector will have a significantly reduced material budget, extend the tracking capabilities to much lower values of transverse momentum and improve the track position resolution at the primary vertex by a factor of 3 as well as the limited read-out rate capabilities.

\subsubsection{TPC}

The TPC is the main central barrel tracking device that surrounds the ITS and covers the pseudo-rapidity range of $|\eta|<0.9$ for tracks with full radial track length (matches in ITS, TRD, and TOF detectors) or up to $|\eta|=1.5$ for reduced track length. With the exception of the dead zones the TPC covers the full azimuth. The active volume with an inner radius of about $85 \mathrm{~cm}$, an outer radius of about $250 \mathrm{~cm}$ and an overall length along the beam direction of 500 $\mathrm{cm}$ is filled with $90 \mathrm{~m}^{3}$ of a $\mathrm{Ne}-\mathrm{CO}_{2}-\mathrm{N}_{2}$ gas mixture at atmospheric pressure and is presented in Figure 3.3 [119]. Charged particles traversing the detector ionize the gas that causes ionization electrons to drift to the endplates of the cylinder under the influence of the electric field of $400 \mathrm{~V} / \mathrm{cm}$ provided by a conducting electrode at the center of the TPC. The precise measurement of the arrival point of drifting electrons in the cylinder plane together with an accurate measurement of their arrival time allows determining the complete trajectory in space of all charged particles inside the TPC. The design parameters of the TPC including the readout chambers, electronics and data handling were optimized to cope with very high particle multiplicities that can reach up to 20000 tracks per single collision in the TPC acceptance [119]. 


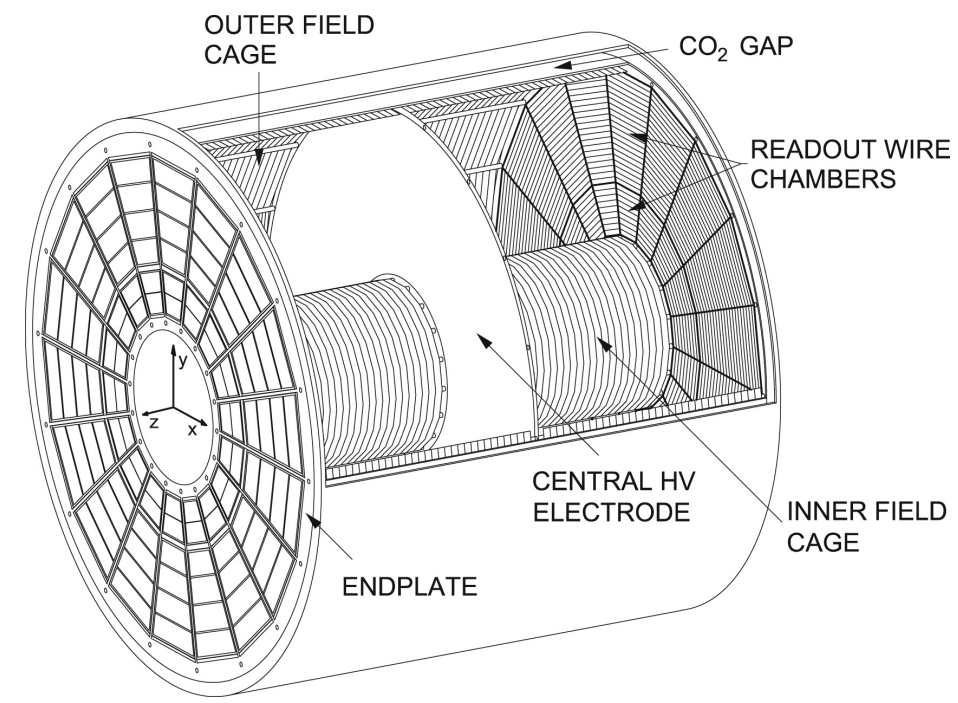

FiguRE 3.3: 3D view of the TPC field cage

In addition to tracking, the TPC provides particle identification over a wide momentum range up to momenta of the order of $50 \mathrm{GeV} / \mathrm{c}$ via simultaneous measurements of the specific energy loss per unit length $(\mathrm{dE} / \mathrm{dx})$, charge and momentum of each particle [115]. The charge in the TPC can be measured for every track on up to 159 pad-rows. However, for the $\mathrm{dE} / \mathrm{dx}$ measurement the truncated mean over $65 \%$ lowest-charge pad-row outputs is used to reduce the fluctuations originating from Landau tail developing towards the higher values in the distribution of the deposited charge [117]. The mean ionization energy loss $\langle\mathrm{dE} / \mathrm{dx}\rangle$ for a given particle type is often described by the Bethe-Bloch formula [120] as following:

$$
-\left\langle\frac{d E}{d x}\right\rangle=\frac{4 \pi}{m_{e} c^{2}} \frac{n z^{2}}{\beta^{2}}\left(\frac{e^{2}}{4 \pi \epsilon_{0}}\right)^{2}\left[\ln \left(\frac{2 m_{e} c^{2} \beta^{2}}{I\left(1-\beta^{2}\right)}\right)-\beta^{2}\right],
$$

where $m_{e}$ is the mass of electron, $c$ is the speed of light, $z$ is the charge of the particle, $\beta$ is its velocity, e is the elementary charge, $\epsilon_{0}$ is the vacuum permittivity, $\mathrm{n}$ is the density of electrons in the material and $\mathrm{I}$ is the mean excitation energy.

In this analysis a simplified Bethe-Bloch parametrization is used for the calculation of the mean ionization energy loss based on a function proposed by the ALEPH experiment [121] :

$$
f(\beta \gamma)=\frac{P_{1}}{\beta^{P_{4}}}\left(P_{2}-\beta^{P_{4}}-\ln \left(P_{3}+\frac{1}{(\beta \gamma)^{P_{5}}}\right)\right),
$$

where $\gamma$ is the Lorentz factor, $\beta$ is the velocity of the particle and $\mathrm{P}_{1-5}$ are the fit parameters that depend on detector properties such as the used gas mixture. The 
decisive quantity used for the particle identification is then the resolution $\sigma_{\mathrm{dE} / \mathrm{dx}}$ and the corresponding selection method is described in more detail in Section 4.2. The estimated resolution of the $\mathrm{dE} / \mathrm{dx}$ measurement slightly depends on the charged particle density and changes from $5.5 \%$ for pp events to $6.5 \%$ for central $\mathrm{Pb}-\mathrm{Pb}$ collisions.

The measured $\mathrm{dE} / \mathrm{dx}$ as a function of particle momentum in the TPC together with the lines corresponding to the aforementioned parametrization is presented in Figure 3.4 [115]. A clear separation between the different particle species can be seen over a wide momentum range. The largest separation is achieved at low $\mathrm{p}_{\mathrm{T}}\left(\mathrm{p}_{\mathrm{T}} \lesssim 0.7 \mathrm{GeV} / \mathrm{c}\right)$ but a good separation is also present in the relativistic rise region $\left(\mathrm{p}_{\mathrm{T}} \gtrsim 0.7 \mathrm{GeV} / \mathrm{c}\right)$ up to $\sim 20 \mathrm{GeV} / \mathrm{c}$. At these higher momenta $\left(\mathrm{p}_{\mathrm{T}} \gtrsim 1\right.$ $\mathrm{GeV} / \mathrm{c}$ ) where particles can not be identified on a track-by-track basis, they can still be separated on a statistical basis via multi-Gaussian fits [115].

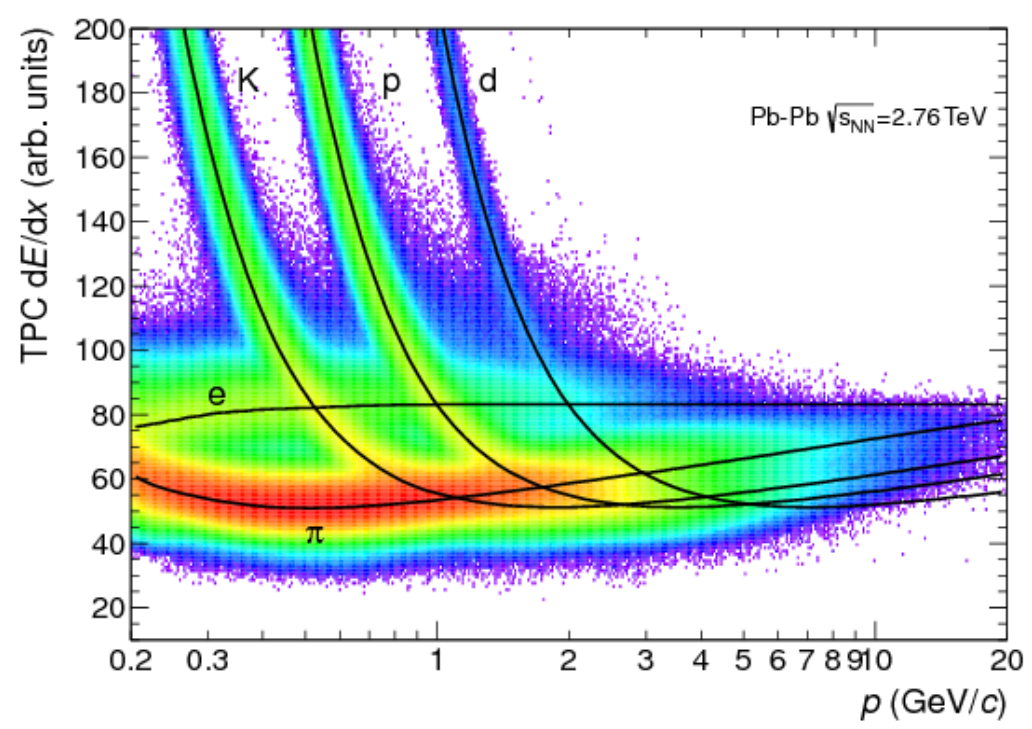

Figure 3.4: Specific energy loss $\mathrm{dE} / \mathrm{dx}$ in the TPC as a function of particle momentum in $\mathrm{Pb}-\mathrm{Pb}$ collisions at $\sqrt{\mathrm{s}_{\mathrm{NN}}}=2.76 \mathrm{TeV}$. The Bethe-Bloch parametrizations of the expected mean energy loss are shown by lines

The data rate provided by the current TPC is limited for central $\mathrm{Pb}-\mathrm{Pb}$ collision due to the current readout system. The replacement of the existing readout chambers with the new technology in the TPC upgrade will increase the readout rate by about two orders of magnitude giving access to previously inaccessible physics observables [122]. 


\subsubsection{TOF}

The TOF detector is located at about $3.7 \mathrm{~m}$ from the beam axis covering the pseudo-rapidity range of $|\eta|<0.9$ as well as the full azimuth. It has a modular structure corresponding to 18 sectors in $\phi$ and to 5 segments in the $\mathrm{z}$ direction. The five modules in a row are located inside a supermodule framework of longitudinal and transverse aluminium beams for each of the 18 sectors as shown in Figure 3.5 [117]. The basic unit of the device is The Multigap Resistive Plate Chamber (MRPC) strip. The TOF consists of 1593 such strips all having the same structure and width $(128 \mathrm{~cm})$ but different length and angle of the strips with respect to the axis of the cylinder. Such a design allows to obtain the full active area with no geometrical dead zones. The only dead area is caused by the presence of the necessary supporting spaceframe structure [117].

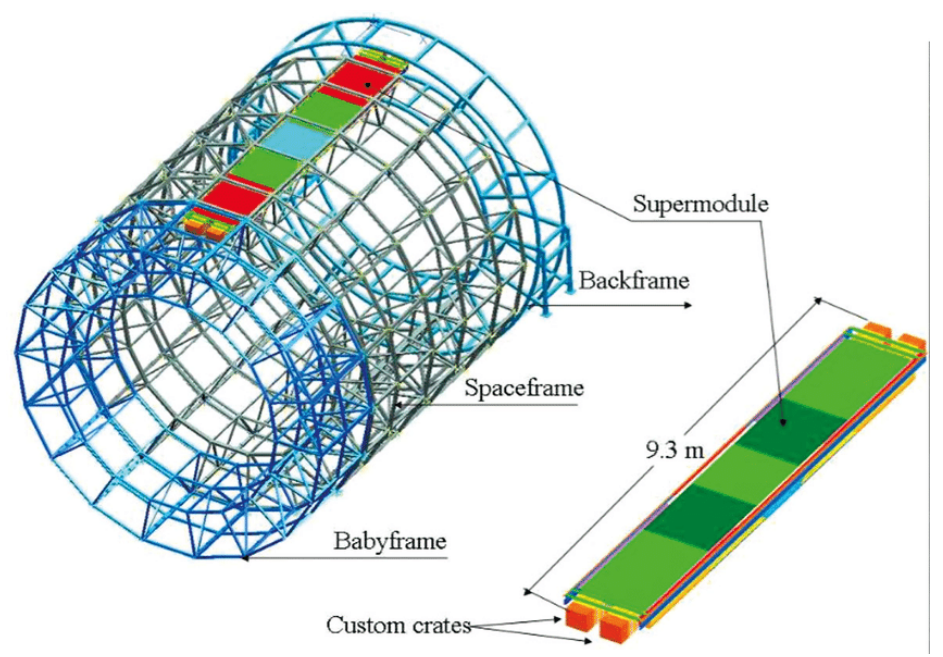

FIgURE 3.5: Schematic drawing of the TOF supermodule

The TOF estimation is based on calculating the difference between the time measurement made by the detector and time of the primary interaction. The latter is usually provided by the T0 detector which consists of two arrays of Cherenkov counters T0C and T0A. However, it can be also estimated from the TOF detector itself if the amount of tracks reaching the TOF is enough to perform a global time minimization, or taken as the average initial time of the LHC fill. The time resolution provided by the MRPC detectors is better than 50 ps [123].

The TOF provides PID in the intermediate momentum range, up to $2.5 \mathrm{GeV} / \mathrm{c}$ for pions and kaons and up to $4 \mathrm{GeV} / \mathrm{c}$ for protons [115]. Combining the measurement of particle's time of flight over a given distance along the track trajectory L 
and its momentum (from the ITS and the TPC) allows identifying the particle's velocity:

$$
\beta=\frac{v}{c}=\frac{L}{t c} .
$$

Through the velocity dependence on mass given by

$$
\beta=\frac{1}{\sqrt{\left(\frac{m c}{p}\right)^{2}+1}}
$$

it's then possible to calculate the mass of the particle and thus identify its type. The example of the measured velocity $\beta$ distribution as a function of momentum measured in the TPC in Pb-Pb collisions is shown in Figure 3.6 [115]. A particle is identified if the position of the corresponding point in this plot is in agreement with the mean value provided by the theoretical curve for a certain particle type given by Equation 3.4 within the measurement errors. The empty band below $300 \mathrm{MeV} / \mathrm{c}$ is present because of no data available in this region due to the fact that the particles do not reach the detector. The background is due to tracks that are incorrectly matched to TOF hits.

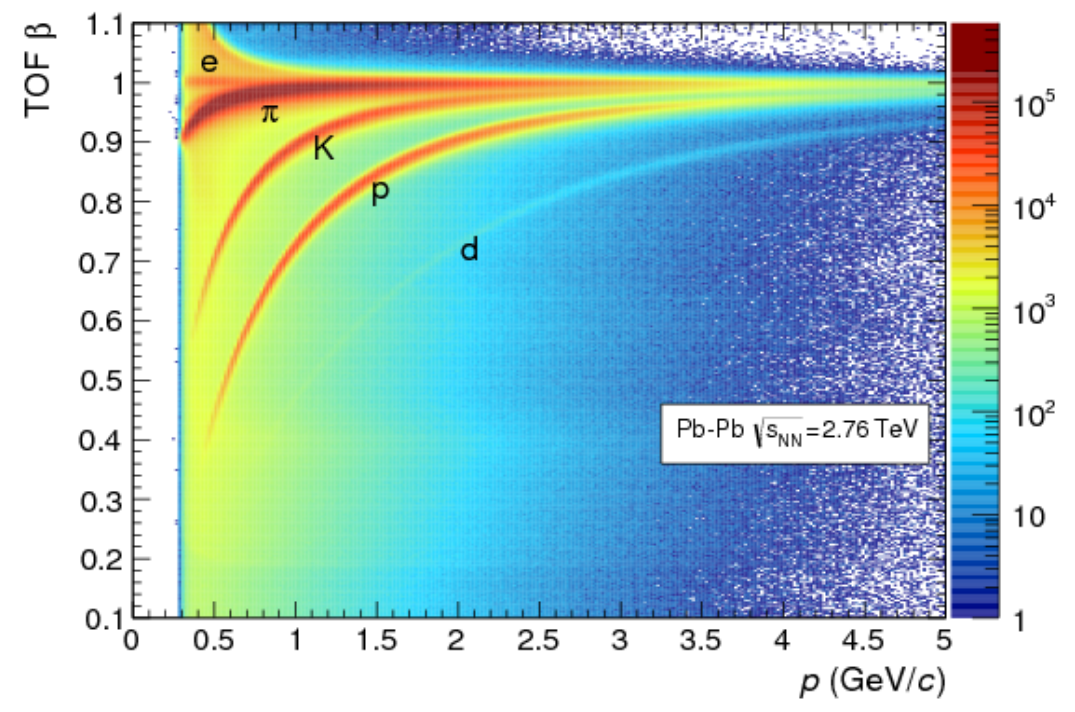

Figure 3.6: Distribution of $\beta$ measured by the TOF detector as a function of momentum for particles reaching the TOF in $\mathrm{Pb}-\mathrm{Pb}$ collisions

At higher transverse momenta where the TOF resolution does not permit trackby-track identification, a fit of multiple Gaussian peaks is used to determine the particle yields [115]. 
The current TOF readout system is limited by several factors. These limitations will be removed in the ongoing upgrade of the TOF data acquisition (DAQ) scheme [124].

\subsubsection{V0}

The V0 detector consists of two arrays of scintillators called V0A and V0C installed on opposite sides of the ALICE LHC beam interaction point covering the pseudorapidity ranges of $2.8<\eta<5.1$ (V0A) and $-3.7<\eta<-1.7$ (V0C). The V0A is located $329 \mathrm{~cm}$ from the nominal vertex $(\mathrm{z}=0)$ on the opposite side to the muon spectrometer. The V0C is fixed on the front face of the hadronic absorber at $90 \mathrm{~cm}$ from the vertex. Each of the V0 arrays is segmented into 32 channels arranged in 4 rings and 8 sectors of $45^{\circ}$ as shown in Figure 3.7 [125]. Each of the channels measures the particle charge and the time of the particle arrival with time resolution being better than $1 \mathrm{~ns}$ independently of the collision system [125].
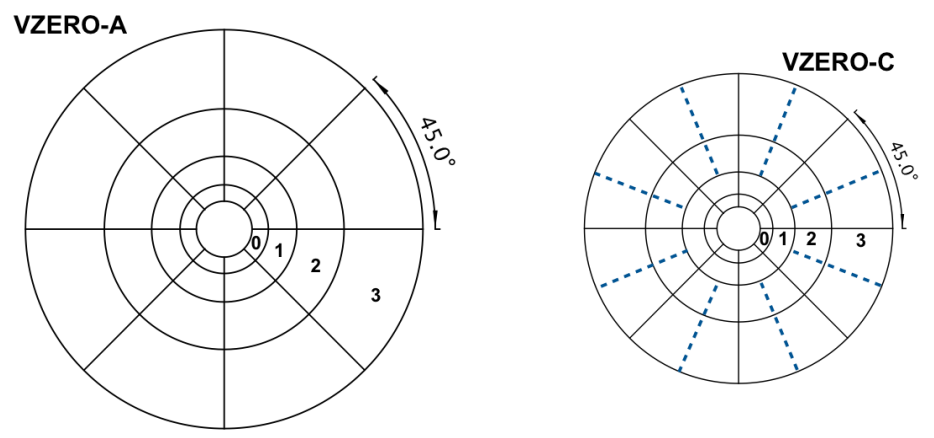

FigURE 3.7: V0A and V0C arrays segmentation

The V0 detector's primary role is to provide minimum-bias and multiplicity triggers in pp and A-A collisions and centrality based triggers in A-A collisions. The centrality determination is obtained via the measurement of multiplicity event-by-event [126]. The example of such measurement and the corresponding centrality classes for $\mathrm{Pb}-\mathrm{Pb}$ collisions at $\sqrt{\mathrm{S}_{\mathrm{NN}}}=2.76 \mathrm{TeV}$ in LHC Run 1 where the V0A and V0C signal coincidence was required for triggering is shown in Figure 3.8 [115]. Moreover, the V0 is used for luminosity and beam-induced background measurements due to the high resolution of the provided time of flight that allows distinguishing particles coming from collisions and particles coming from beam-gas backgrounds. 


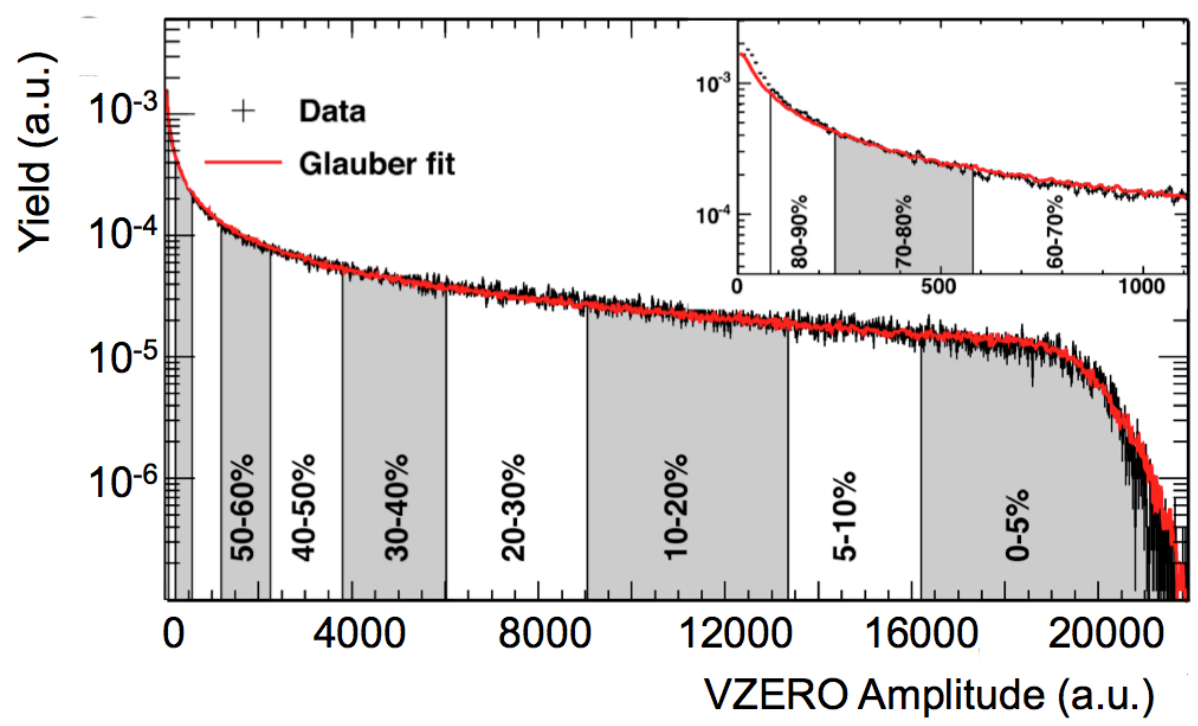

Figure 3.8: Sum of amplitudes in the two V0 arrays (black histogram) in $\mathrm{Pb}-\mathrm{Pb}$ collisions at $\sqrt{\mathrm{S}_{\mathrm{NN}}}=2.76 \mathrm{TeV}$. The defined centrality classes of the collision are shown with the shaded areas 



\section{Chapter 4}

\section{Analysis Details}

\subsection{Data samples, event and track selection}

\subsubsection{Data samples}

The analysis presented in this thesis has been performed on proton-proton data at $\sqrt{\mathrm{s}}=5.02 \mathrm{TeV}$ collected with the ALICE detector in 2017 during LHC Run 2. Two data samples recorded during different periods were used: LHC17p and LHC17q. The LHC17q has a larger interaction rate with respect to the LHC17p.

Moreover, two different detector readout configurations indicated from now on as "FAST" and "woSDD" were used in each of the periods resulting in four final samples used in the analysis: LHC17p_pass1_FAST, LHC17p_pass1_CENT_woSDD, LHC17q-pass1_

FAST and LHC17q_pass1_CENT_woSDD. The choice of these configurations was driven by the need to account for a large amount of busy time failures in the SDD detector during the recording. The FAST configuration provides the trigger that becomes active during the busy time in the SDD. Therefore, the SDD was not present in the readout while the rest of the detectors were recording data, so all events collected with this configuration have no SDD information. The woSDD sample provides all events with the SDD detector in the readout, but its information is excluded later at the level of the track reconstruction. These two samples are fully independent which allows us to combine them for the final result. 


\subsubsection{Event selection}

As a first step in the event selection procedure the so-called kINT7 online minimum bias trigger was used. This corresponds to the coincidence of particle hit signals in both V0A and V0C detectors. Additionally, the events coming from background beam-gas interactions were removed by applying an offline selection. Such interactions can occur between the beams and the residual gas within the beam pipe or accelerator material [125]. The signal produced by background is detected earlier compared to the time corresponding to a collision in the nominal interaction point that allows a clear distinguishing of both types using fast timing detectors such as the V0 [115].

Another kind of background in the event selection procedure might come from the overlap of the collisions, referred to as pile-up. Two pile-up types are distinguished, i.e. in-bunch and out-of-bunch pile-up. First kind of pile-up occurs when multiple collisions happen close in time in the same bunch crossings and their vertices are not separable. Second one occurs when one (or more) collisions happen in a different bunch crossing from the one which triggered the acquisition. If the detector readout time is longer than the difference in time between such collisions, they might be recorded as one. To reject the events coming from both pile-up types in this analysis, a pile-up tagging algorithm based on multiple vertices was applied [127]. In a default algorithm called track vertexer, global tracks reconstructed in ITS and TPC, complemented by TOF points if available, were used to identify different vertices within the same read-out window. Only tracks within the same bunch crossing according to TOF were allowed to contribute to the same vertex. If more than one vertex was found, the vertex with largest amount of contributors among all the vertices was defined as a primary vertex. As a variation for the systematic uncertainty evaluation described in Section 5.1.2, an SPD pile-up tagging algorithm (SPD vertexer) for same-bunch or different-bunch crossing (within the SPD readout time of $300 \mathrm{~ns}$ ) removal was used instead. In this algorithm only local reconstruction of the SPD tracklets (lines connecting pairs of track hits in each SPD layer) is required. They are used to localize the primary interaction vertex by searching for the maximum number of tracklets pointing to the same location. If more than one vertex satisfying some criteria defined by the user such as, for example, minimum number of contributors (i.e. tracklets) to the pile-up candidate vertices, is present in the event, this event is tagged as pile-up and is eventually rejected. Since the SPD contribution to used tracks in the default algorithm with tracks is not obligatory, the track vertexer has wider time coverage than the SPD vertexer. 
Finally, only events with z position of the primary vertex within $10 \mathrm{~cm}$ from the nominal interaction point were selected.

After applying all the aforementioned selection criteria, approximately 690 millions of events were analyzed in the $0-80 \%$ multiplicity range. Multiplicity selection was performed using the V0A estimator. The multiplicity classes were defined by integrating the measured V0A amplitude distribution shown in Figure 4.1 similarly to what is done for the $\mathrm{Pb}-\mathrm{Pb}$ case (see Figure 3.8). The last 80-100\% multiplicity bin was not used due to the peculiar behaviour not only in terms of statistics but also physics-wise because of the significant contribution from diffractive events.

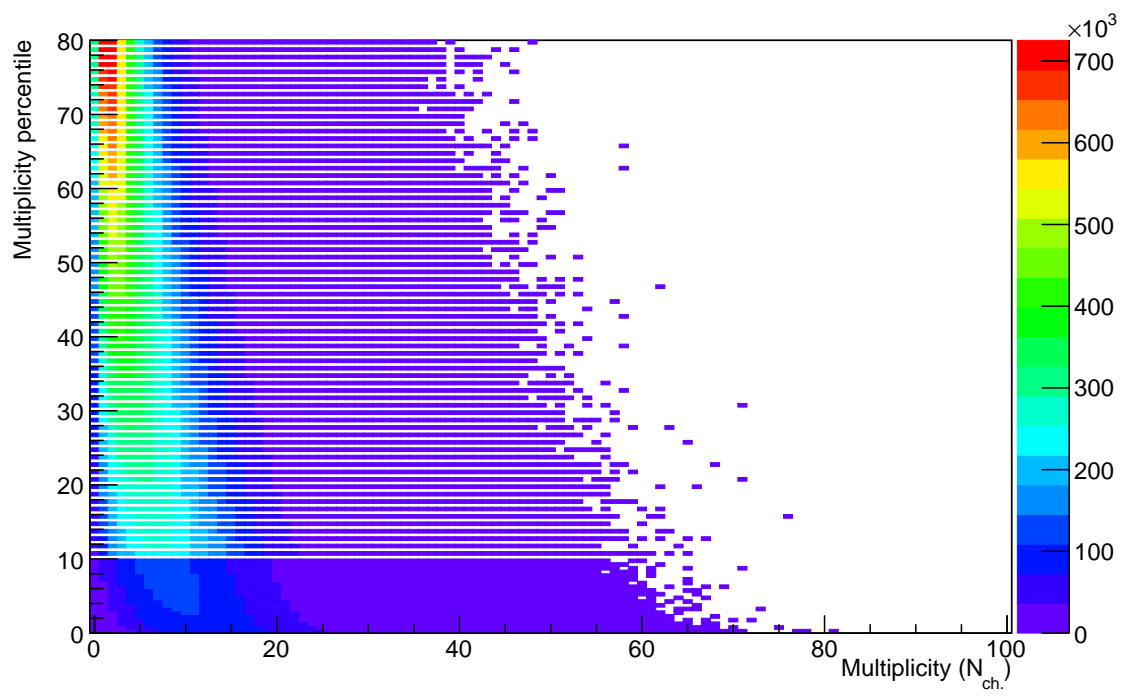

FIGURE 4.1: Multiplicity percentile as a function of charged particle multiplicity provided by V0A estimator in pp collisions at $\sqrt{\mathrm{s}}=5.02 \mathrm{TeV}$

\subsubsection{Track selection}

The selection of primary tracks for this analysis was performed by using combined information from the TPC and the ITS detectors, the two main tracking detectors of ALICE.

The ALICE procedure of track finding begins from the two outer TPC pad rows where the coordinates of the crossing points (space points) between tracks and detector sensitive elements are calculated. The track fitting (reconstruction) based on the Kalman filter approach [128] is then started using these two space points and the primary vertex point. Subsequently, tracks are propagated to the inner TPC radius and to each ITS layer towards the primary vertex and then backwards through the ITS, the TPC and the TRD matching also with available hits reconstructed in the TOF detector and other outer ALICE detectors. In 
the final stage of the reconstruction the refit of the tracks is done in the inward direction once again to obtain the best determination of the track parameters by improving the information from the previous iterations. After that the resulting quality parameter of the track fit $\chi^{2}$ is obtained.

The TPC readout chambers have a total number of 159 pad rows where the signal by a traversing particle can be produced. Therefore, to select high-quality tracks they were required to have at least 70 reconstructed space points in the TPC and a maximum value of $\chi^{2}$ per TPC reconstructed point of 4 . Additionally, to reduce the contamination from secondary particles, the distance of closest approach (DCA) of the track to the primary vertex in the transverse plane $\left(\mathrm{DCA}_{\mathrm{xy}}\right)$ and along the beam direction $\left(\mathrm{DCA}_{\mathrm{z}}\right)$ were used. Since secondaries are produced at the secondary vertices far away from the primary vertex, their $\mathrm{DCA}_{\mathrm{xy}}$ distribution will be broader with respect to the one of primary particles that is peaked at 0 as seen in Figure 4.2. Only tracks that passed a tight $\mathrm{p}_{\mathrm{T}}$ dependent requirement on $\mathrm{DCA}_{\mathrm{xy}}\left(\mathrm{DCA}_{\mathrm{xy}}<0.0105+0.0350 / \mathrm{p}_{\mathrm{T}}\right)$ as well as a non $\mathrm{p}_{\mathrm{T}}$-dependent one in longitudinal direction $\left(\mathrm{DCA}_{\mathrm{z}}<2 \mathrm{~cm}\right)$ were accepted.

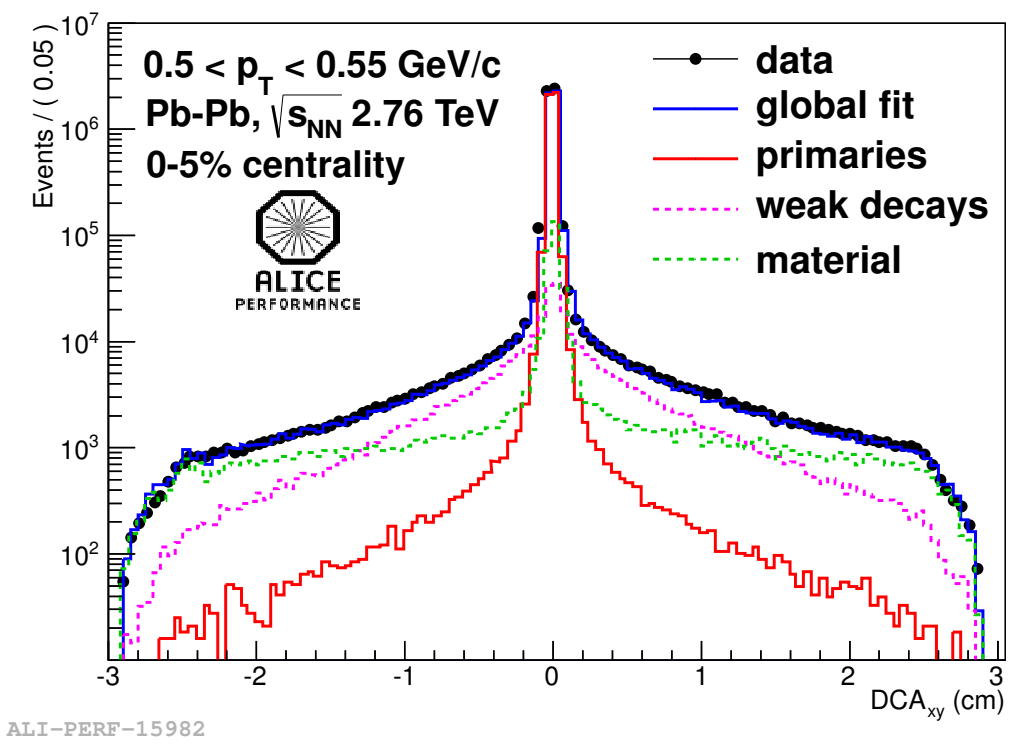

FigurE 4.2: Transverse DCA $_{\mathrm{xy}}$ distribution of charged particles in most central $\mathrm{Pb}-\mathrm{Pb}$ collisions

Applying these selection criteria does not exclude all secondary tracks, therefore the remaining contamination is defined as the number of secondary tracks, coming from weak decay or interaction with the material, divided by the total number of generated particles (primaries and secondaries). Moreover, since part of the tracks can be lost due to the detector geometry such as the dead or intersection zones as well as due to the physical processes where particles can 
get absorbed, not all the tracks will be reconstructed. This introduces a limited tracking efficiency $\epsilon$ that is defined as the number of reconstructed tracks divided by the number of generated particles. Both terms are extracted from Monte Carlo simulation. In case of identified species analysis different efficiencies and contamination from secondaries are expected for different particles due to their different interaction cross sections with the material. Therefore, this weight is computed separately for all particle types.

In this analysis the LHC17p_pass1_FAST sample produced by the PYTHIA8 Monte Carlo generator [129] was used to estimate the aforementioned components of the correction factor. It incorporates the detector conditions matching the ones of the data samples described in Section 4.1.1. As an example, the $\mathrm{p}_{\mathrm{T}}$, multiplicity and charge dependence of the tracking efficiency and contamination from secondaries for pions that passed the track selection criteria can be seen in Figures 4.3 and 4.4, respectively. Blue color shows the result for positive, while orange for negative tracks. Each series of points corresponds to a multiplicity interval changing from darker color representing the highest multiplicity class, up to the lightest color, representing the lowest multiplicity class. It can be observed that the efficiency increases sharply with increasing transverse momentum until it reaches values of $\approx 85 \%$ around $1 \mathrm{GeV} / \mathrm{c}$. As for the contamination, the contribution of secondaries is not very significant due to the effective secondary track rejection cuts including the tight $\mathrm{p}_{\mathrm{T}}$-dependent cut on the track's $\mathrm{DCA}_{\mathrm{xy}}$.
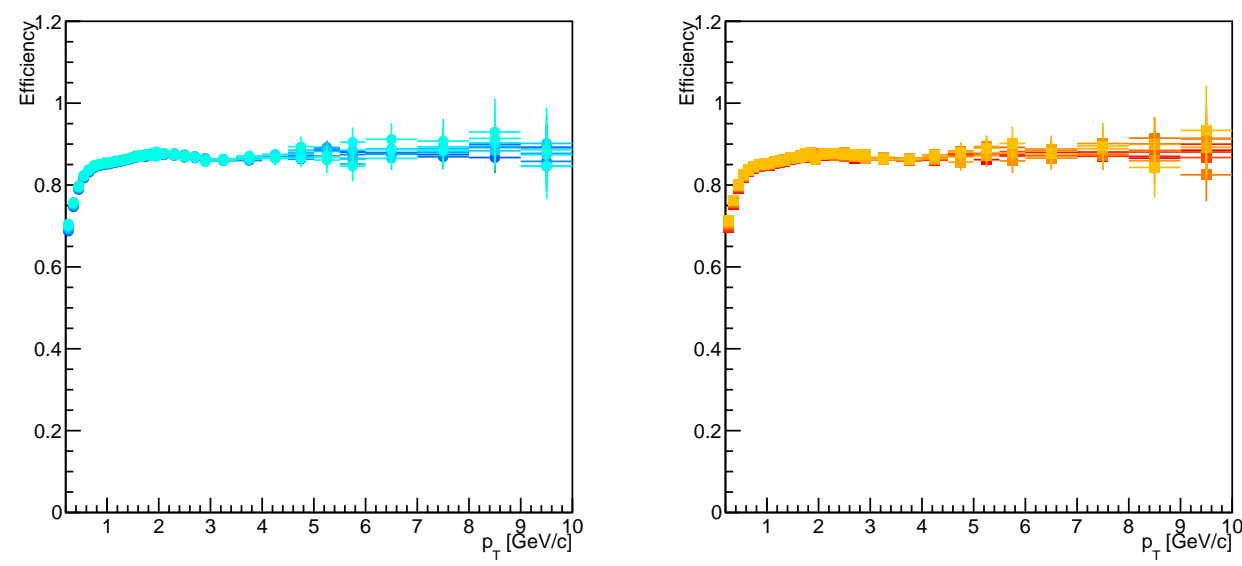

Figure 4.3: Tracking efficiency as a function of transverse momentum for positive (blue) and negative (orange) pions 

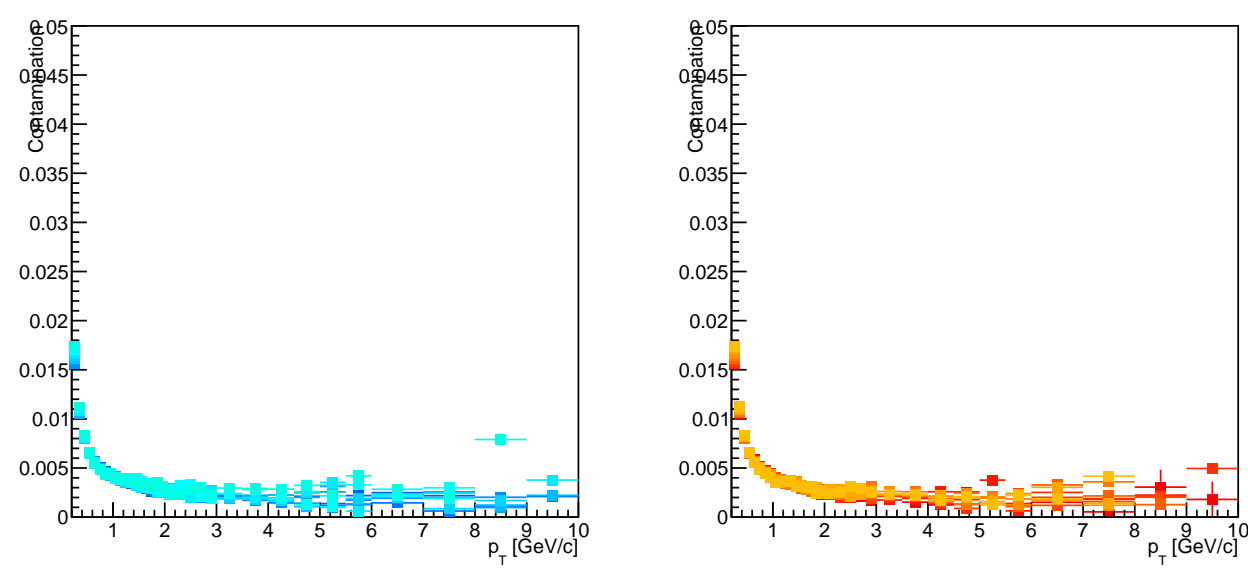

Figure 4.4: Contamination from secondary particles as a function of transverse momentum for positive (blue) and negative (orange) pions

Apart from single particle cuts mentioned above, two-particle cut on the closest azimuthal distance $\Delta \varphi^{*}$ between two tracks in the TPC volume was applied [111]:

$$
\Delta \varphi^{*}=\varphi_{1}-\varphi_{2}-q_{1} \cdot \arcsin \left(\frac{0.0075 B_{z} r}{p_{T_{1}}}\right)+q_{2} \cdot \arcsin \left(\frac{0.0075 B_{z} r}{p_{T_{2}}}\right)
$$

where $\varphi_{1}$ and $\varphi_{2}$ are the azimuthal angles, $\mathrm{p}_{\mathrm{T}_{1}}$ and $\mathrm{p}_{\mathrm{T}_{2}}$ are the transverse momenta, $\mathrm{q}_{1}$ and $\mathrm{q}_{2}$ are the charges of the two tracks forming the particle pair, $\mathrm{B}_{\mathrm{z}}$ is the magnetic field in the $\mathrm{z}$ direction and $\mathrm{r}$ is the radius of the TPC (between $0.8<\mathrm{r}<2.5 \mathrm{~m})$. This was done in order to reduce the contribution from track splitting (when signal produced by one track is incorrectly reconstructed as two tracks) or/and track merging (when two nearby tracks are reconstructed as one track). The default cut used in the analysis was $\Delta \varphi^{*}<0.02$. The variation of this cut was studied to account for the possible contribution to the systematic uncertainty that is described in Chapter 5.

All tracks were selected from the low $\mathrm{p}_{\mathrm{T}}$ range of $0.2-2 \mathrm{GeV} / \mathrm{c}$ and for the midrapidity range of $|y|<0.5$. The latter selection allows for a fair comparison of the results of the balance function for different particle species. This was done since the bigger the mass of the particle, the less statistics is available at the edges of acceptance that leads to significant fluctuations around the edges of $\Delta y$ in the final results. 


\subsection{PID strategy}

In this analysis three particle species are studied: pions, kaons and protons. The particle identification (PID) is performed using what is referred to as the no approach, where n $\sigma$ is defined as the deviation of the measured signal $\mathrm{S}$ from the expected one $\mathrm{E}$ for a given particle type $\mathrm{i}$ in terms of the detector resolution $\sigma$ :

$$
n \sigma_{\alpha}^{i}=S_{\alpha}-E_{\alpha}^{i}
$$

with $\alpha$ referring to the detector type.

This analysis is based on the PID information provided by the TPC and the TOF detectors. In case of the TPC measurement, $\mathrm{n}$ is given by the number of standard deviations $(\sigma)$ from the theoretical parameterization given by the Bethe-Bloch formula (see Equation 3.2) of the measured ionisation energy loss $(\mathrm{dE} / \mathrm{dx})$ of a particle track in the TPC. In case of the TOF, it is the deviation from the expected arrival time for a given species in the detector. Combining the information from both detectors allows to improve the purity and the momentum reach of the identified species.

Since n $\sigma$ reflects the probability that a track is of a particular particle type, the selection in this method is based on a "true/false" decision. A certain identity is assigned to a track if its no value lies within a certain range around the expectation defining whether a particle belongs to a certain type or not. Figure 4.5 shows the example of pion no measured with TOF in this analysis before applying the PID selection criteria. As it can be seen, most of pions are located within $3 \sigma$ range from the peak of their distribution. For this characteristic momentum range, it can be seen that there is a small contamination in the sample of pions originating from kaons. At the same time, protons are very well separated from the other two species. Therefore, to separate different particle types from each other the acceptance n $\sigma$ cuts usually select the tracks with n $\sigma$ values within 2 or $3 \sigma$ range from the Gaussian peak, while rejection cuts select the values outside of this range. 


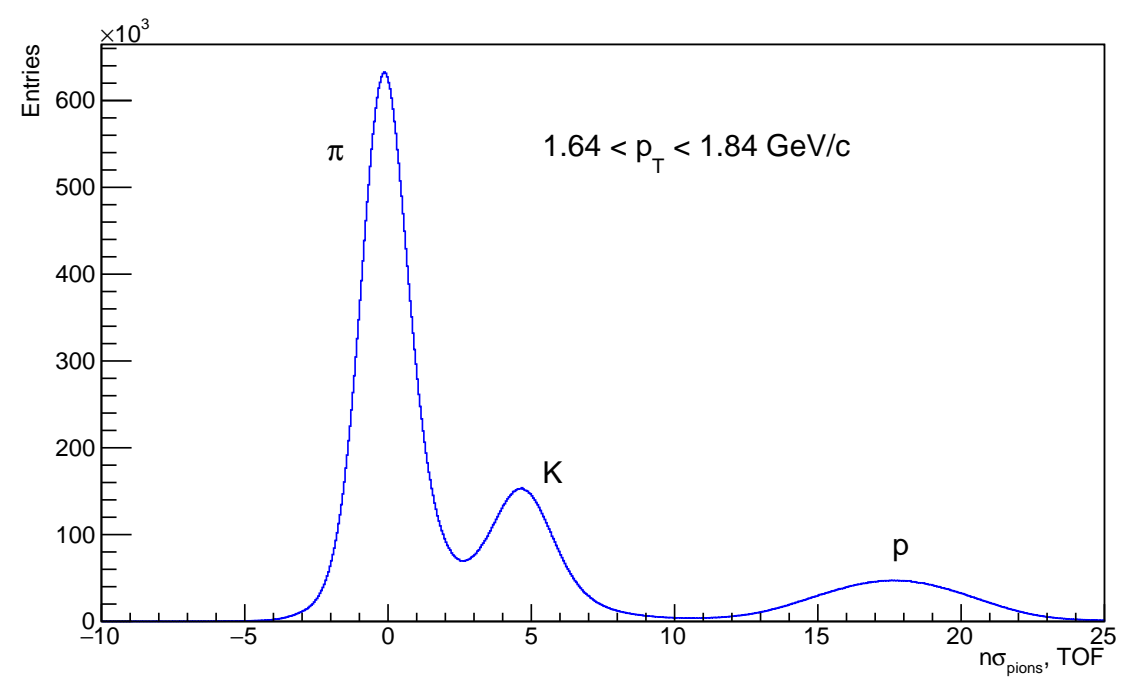

Figure 4.5: TOF n $\sigma$ signal for pions in $1.64<\mathrm{p}_{\mathrm{T}}<1.84 \mathrm{GeV} / \mathrm{c}$

The selection in this analysis is done for pions and kaons starting from 0.2 $\mathrm{GeV} / \mathrm{c}$ and for protons starting from $0.5 \mathrm{GeV} / \mathrm{c}$ (due to the large secondary contamination in the low $\mathrm{p}_{\mathrm{T}}$ ) up to $2 \mathrm{GeV} / \mathrm{c}$ using the following no cuts for two ranges of transverse momentum:

- $p_{T}<0.5 \mathrm{GeV} / \mathrm{c}$ :

\begin{tabular}{|c|c|c|c|}
\hline particle type & $\left|\mathrm{n} \sigma_{\mathrm{TPC}_{\pi}}\right|$ & $\left|\mathrm{n} \sigma_{\mathrm{TPC}_{\mathrm{K}}}\right|$ & $\left|\mathrm{n} \sigma_{\mathrm{TPC}_{\mathrm{p}}}\right|$ \\
\hline pions & $<2$ & $>3$ & $>3$ \\
\hline kaons & $>3$ & $<2$ & $>3$ \\
\hline protons & $>3$ & $>3$ & $<2$ \\
\hline
\end{tabular}

- $p_{T}>0.5 \mathrm{GeV} / \mathrm{c}$ :

\begin{tabular}{|c|c|c|c|}
\hline particle type & $\left|\mathrm{n} \sigma_{\mathrm{comb}_{\pi}}\right|$ & $\left|\mathrm{n} \sigma_{\mathrm{comb}_{K}}\right|$ & $\left|\mathrm{n} \sigma_{\mathrm{comb}_{\mathrm{p}}}\right|$ \\
\hline pions & $<2$ & $>3$ & $>3$ \\
\hline kaons & $>3$ & $<2$ & $>3$ \\
\hline protons & $>3$ & $>3$ & $<2$ \\
\hline
\end{tabular}

where

$$
n \sigma_{c o m b}=\sqrt{n \sigma_{T P C}^{2}+n \sigma_{T O F}^{2}}
$$

In every step of this strategy apart from the $2 \sigma$ acceptance cut an additional check is performed to make sure that n $\sigma$ of the given track is outside $3 \sigma$ of other 
particle types. This reduces the contamination and enhances the purity of the track sample for a given species.

Applying the aforementioned selection criteria leads to a certain PID contamination $c_{P I D}$ and efficiency $\epsilon_{\text {PID }}$ of a chosen PID strategy. $c_{P I D}$ is the number of wrongly identified particles with a given PID strategy divided by the number of all particles of this type reconstructed within the acceptance, while $\epsilon_{\text {PID }}$ represents the number of particles identified with a given PID strategy for a given particle type, divided by the number of all particles of this type reconstructed within the acceptance.

Similarly as described in Section 4.1.3 for the case of tracking efficiency and secondary contamination, LHC17p_pass1_FAST sample produced by the PYTHIA8 Monte Carlo generator was used to estimate the PID correction terms. An example of an one-dimensional $\mathrm{p}_{\mathrm{T}}$, multiplicity and charge-dependent PID efficiency and PID contamination computed in this analysis is shown in Figure 4.6 for pions and Figure 4.7 for protons, respectively. It can be seen that the efficiency is $100 \%$ at low transverse momentum where only the TPC is used for all the particle types and it decreases to $80 \%$ up to $2 \mathrm{GeV} / \mathrm{c}$ where a $2 \mathrm{n} \sigma_{\text {comb }}$ cut is applied as explained earlier in this section. The further decrease is due to tighter rejection criteria applied at higher transverse momentum values. As for the contamination, the results are shown for protons since for pions the contribution from other particle species is very low with the applied PID strategy. The maximum value is $2 \%$ for low $\mathrm{p}_{\mathrm{T}}$ range of $0.2-2 \mathrm{GeV} / \mathrm{c}$ that demonstrates a great performance of the strategy in providing a very pure sample of a given particle type.
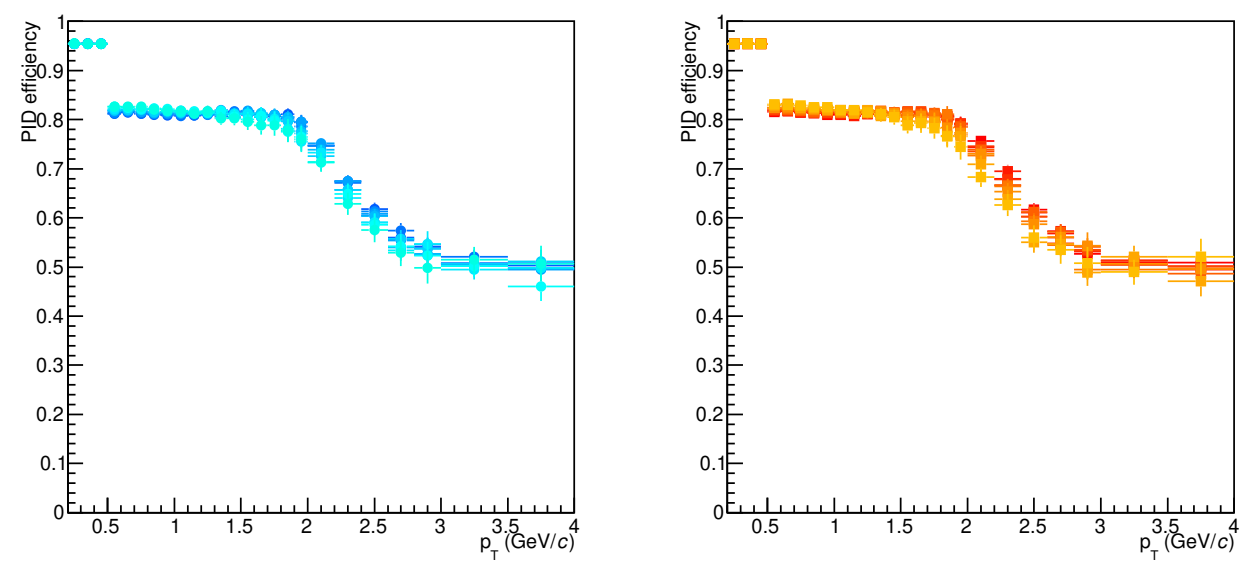

Figure 4.6: PID efficiency as a function of transverse momentum for positive (blue) and negative (orange) pions 

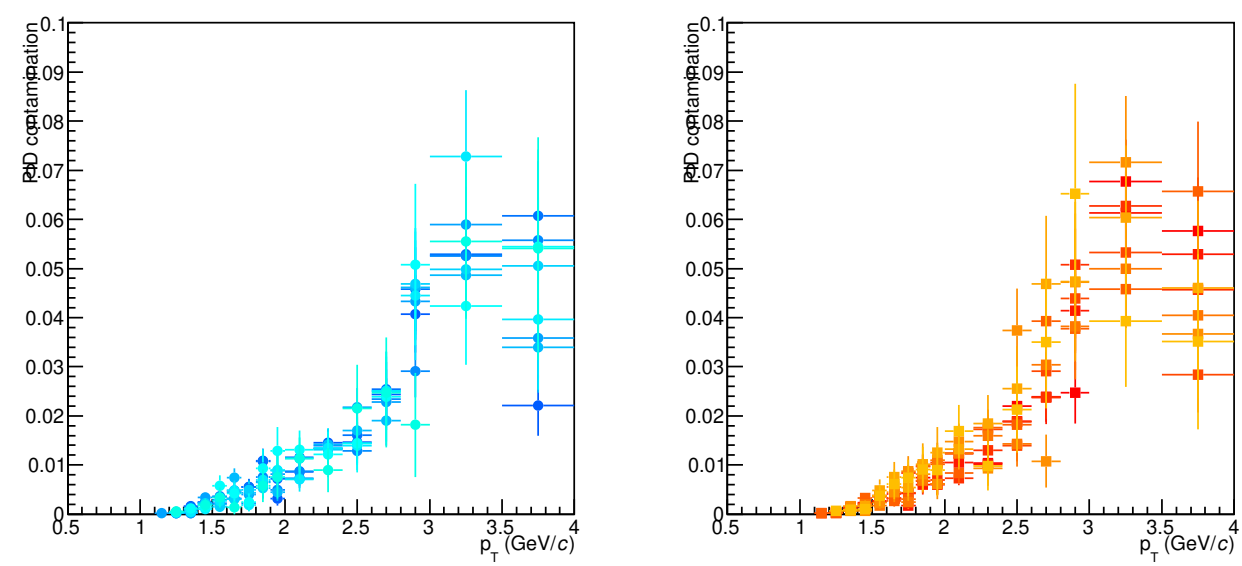

FIGURE 4.7: Contamination from misidentified particles as a function of transverse momentum for positive (blue) and negative (orange) protons

In addition, if a chosen PID strategy uses the TOF detector, a TOF matching efficiency is introduced to account for the loss of the tracks in between the TPC and the TOF. It is calculated for different hadrons as the number of tracks reconstructed in the TPC and the TOF divided by the number of tracks reconstructed only in the TPC. The $\mathrm{p}_{\mathrm{T}}$, multiplicity and charge dependence of this term is shown in Figure 4.8 for pions selected in this analysis. The efficiency is at $100 \%$ below $0.5 \mathrm{GeV} / \mathrm{c}$ since only TPC information is required, while from 0.5 $\mathrm{GeV} / \mathrm{c}$ a small increase in efficiency can be observed starting from around $50 \%$ up to around $65-70 \%$ at $2 \mathrm{GeV} / \mathrm{c}$.
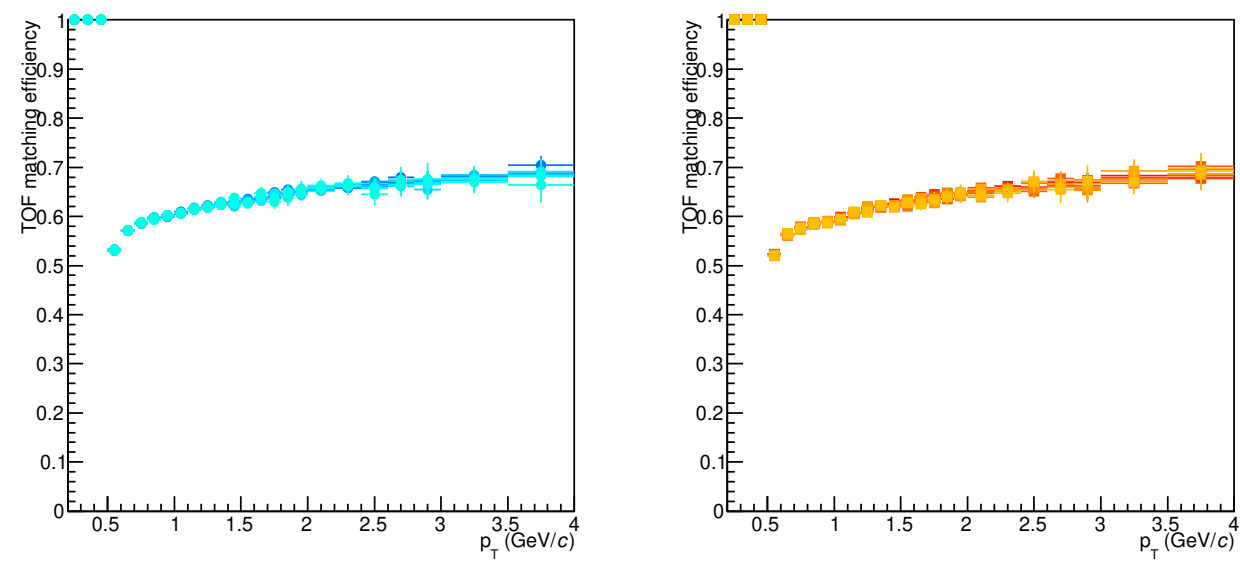

FiguRE 4.8: TOF matching efficiency as a function of transverse momentum for positive (blue) and negative (orange) pions

The choice of using only the TPC at low-p $\mathrm{p}_{\mathrm{T}}(<0.5 \mathrm{GeV} / \mathrm{c})$ is made to exploit the good separation of particle species that come from the energy deposit in the 
TPC gas, but also to limit acceptance effects due to the fact that low-momentum particles hardly reach the TOF.

The performance of the strategy was studied by varying the no requirements and computing the corresponding purity of the sample (or PID contamination) and PID efficiency for each particle type. The results of this study are described in Chapter 5 in the systematic uncertainty section.

\subsection{Analysis method}

The event and track sample selected with the criteria described before was analysed using the balance function (see Chapter 2). The balance function analysis is based on the measurement of the associated per-trigger yield distributions for different charge combinations given by Equation 4.4 for the case of pairs of particles with positive and negative charge. As a first step, a trigger particle is selected within a range of $\mathrm{p}_{\mathrm{T}, \text { trig. values. This trigger particle is combined }}$ with all particles of the same event, referred to from now on as associated particles, that have a transverse momentum value within a range of $\mathrm{p}_{\mathrm{T}, \text { assoc. }}$. When combining and forming pairs, the angular differences, both azimuthal and polar, are calculated i.e. $\Delta \varphi$ and $\Delta \eta$, respectively. The positive-negative associated per-trigger yield $\mathrm{C}_{(+,-)}$is then constructed according to:

$$
C_{(+,-)}=\frac{1}{N_{\text {trig, },}} \frac{d^{2} N_{\text {assoc },-}}{d \Delta \eta d \Delta \varphi}=\frac{S_{(+,-)}}{f_{(+,-)}}
$$

where $\mathrm{N}_{\text {trig,+ }}$ is the number of positive trigger particles, $\mathrm{S}_{(+,-)}$is the signal distribution or the particle pair density calculated from the same event and $f_{(+,-)}$is the correction factor applied to account for particle pair acceptance effects (more on this later in the text). Both $\mathrm{S}$ and $\mathrm{f}$ are computed as an average over all events. The signal distribution, or same event distribution, is given by

$$
S_{(+,-)}=\frac{1}{N_{t r i g,+}} \frac{d^{2} N_{\text {same },(+,-)}}{d \Delta \eta d \Delta \varphi}
$$

where $\frac{\mathrm{d}^{2} \mathrm{~N}_{\text {same, }(+,-)}}{\mathrm{d} \Delta \eta \mathrm{d} \Delta \varphi}$ is the particle pair distribution for positive trigger and negative associated particles from the same event constructed according to the prescription described early in this section. The particle pair acceptance correction term, or mixed event distribution, is defined as 


$$
f_{(+,-)}=\alpha \frac{d^{2} N_{\text {mixed },(+,-)}}{d \Delta \eta d \Delta \varphi}
$$

and is computed by combining the positive trigger and negative associated particles from different, yet similar in terms of multiplicity and z-position of primary vertex $V_{z}$ (for both particles it should be within $\pm 2 \mathrm{~cm}$ of each other), events. This technique is called event mixing. A maximum number of 15 events is used to fill each multiplicity and $\mathrm{V}_{\mathrm{z}}$ bin in order to limit the computational time. Since the mixed event term reflects the probability of reconstructing the pair of particles depending on their location within the detector acceptance, as a next step, it is normalized to unity at $(\Delta \eta, \Delta \varphi)=(0,0)$ by a factor $\alpha$. The example of a normalized distribution is shown in Figure 4.9 [110]. It shows that the highest probability of reconstructing the particle pair is reached if particles $\eta$ values are close, while the larger the separation, the lower is the probability which leads to the final triangular shape. This technique allows to keep only the effects related to the particle pair acceptance while having no physical correlations involved. Therefore, the mixed event distribution is used to correct the same event distribution for two-particle effects such as losses of particle pairs due to the limited detector acceptance and detector inefficiencies.

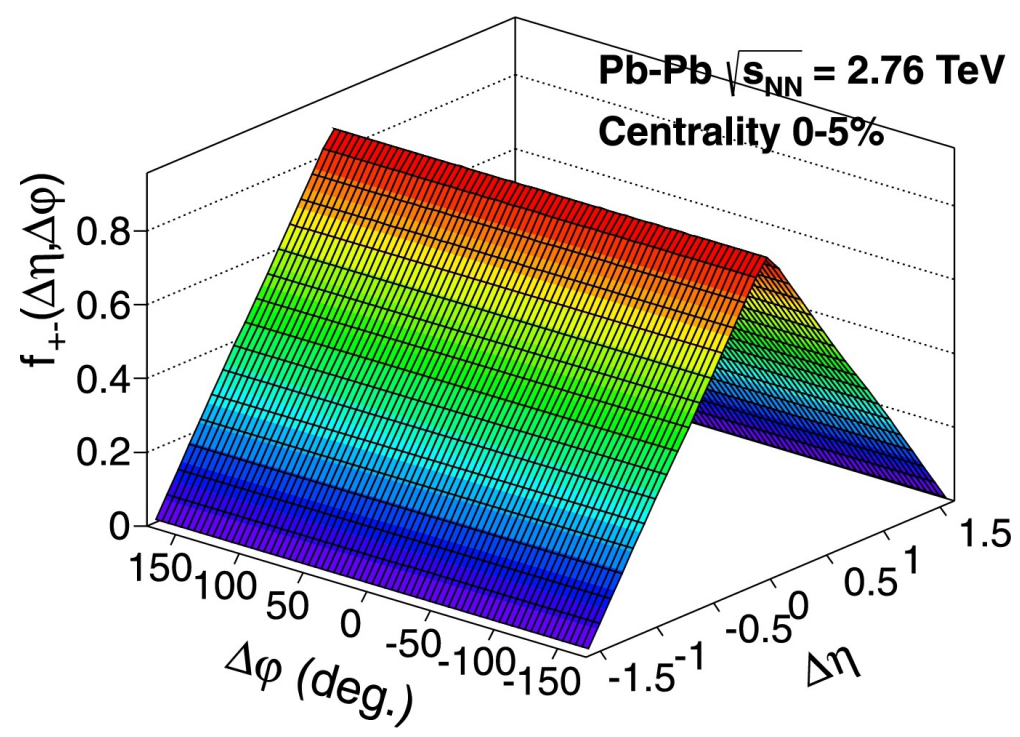

Figure 4.9: The mixed event distribution for $\mathrm{Pb}-\mathrm{Pb}$ collisions in $0-5 \%$ centrality class

Additionally, the inefficiencies of the ALICE detector such as the 18 intersection zones of the TPC detector or the dead channels of the ITS detector can lead to track losses reflected in non-uniform single-particle $\varphi$ distribution. In this case the effect will be also propagated to the two-particle distributions and will be cancelled out at the level of the same event over the mixed event ratio. 
All terms used for the same and mixed event distribution calculation are also corrected for single particle tracking efficiency $\epsilon$ and contamination from secondary particles c described in detail in Section 4.1.3. This is done by computing a weight that is used as a part of the final correction (see Equation 4.10) that is applied on a track by track basis on all particle tracks that form a pair in the pertrigger yield evaluation. The weight is extracted from Monte Carlo simulation and is given by

$$
w_{\text {tracking }}\left(p_{T}\right)=\frac{1-c}{\epsilon} .
$$

The way it develops as a function of $\mathrm{p}_{\mathrm{T}}$, multiplicity and charge is presented in Figure 4.10 for pions selected in this analysis.
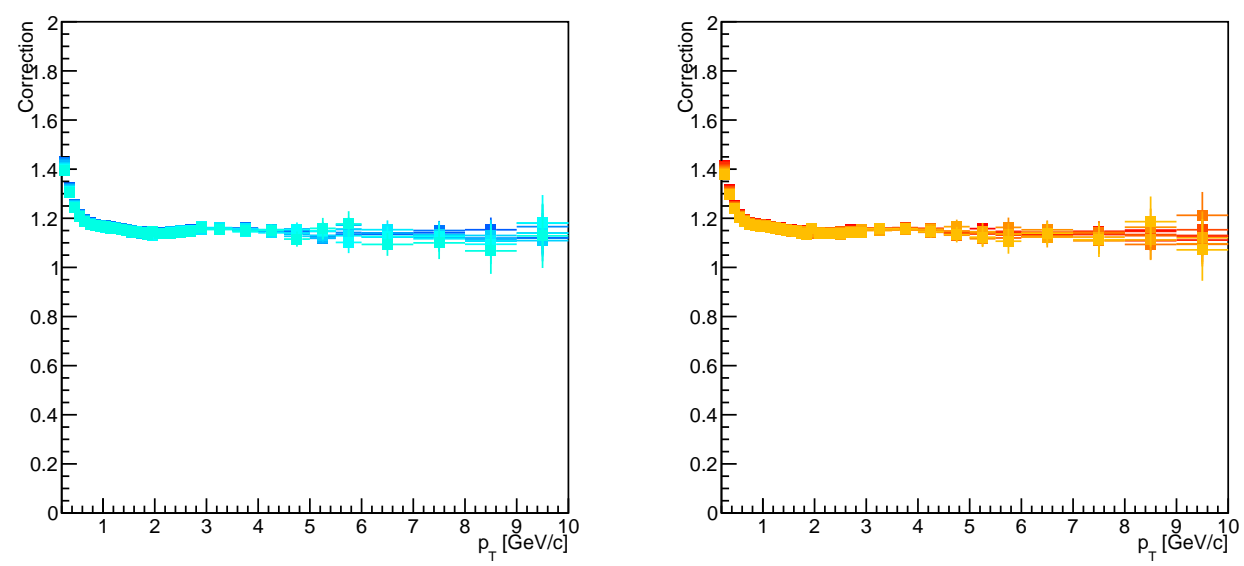

FiguRE 4.10: Correction factor $\mathrm{w}_{\text {tracking }}\left(\mathrm{p}_{\mathrm{T}}\right)$ as a function of transverse momentum for positive (blue) and negative (orange) pions

Additionally, in case of identified particle analysis a correction for TOF matching efficiency, particle identification (PID) efficiency $\epsilon_{\mathrm{PID}}$ and contamination CPID is necessary (see Section 4.2). These corrections are taken into account when forming a final weight given by Equation 4.10 that is applied on all particle tracks that form a pair in the per-trigger yield evaluation. The first one is computed as an inverse of a TOF matching efficiency and is defined as

$$
w_{\text {TOFmatching }}\left(p_{T}\right)=\frac{N_{\text {tracks }_{T P C}}}{N_{\text {tracks }_{T P C+T O F}}}
$$

while two latter terms are combined into a weight that is given by

$$
w_{\mathrm{PID}}\left(p_{T}\right)=\frac{1-c_{P I D}}{\epsilon_{P I D}}
$$


The final weight is computed as

$$
w_{\text {final }}\left(p_{T}\right)=w_{\text {tracking }}\left(p_{T}\right) \cdot w_{\mathrm{PID}}\left(p_{T}\right) \cdot w_{\mathrm{TOFmatching}}\left(p_{T}\right)
$$

and is reported in Figure 4.11 for pions.
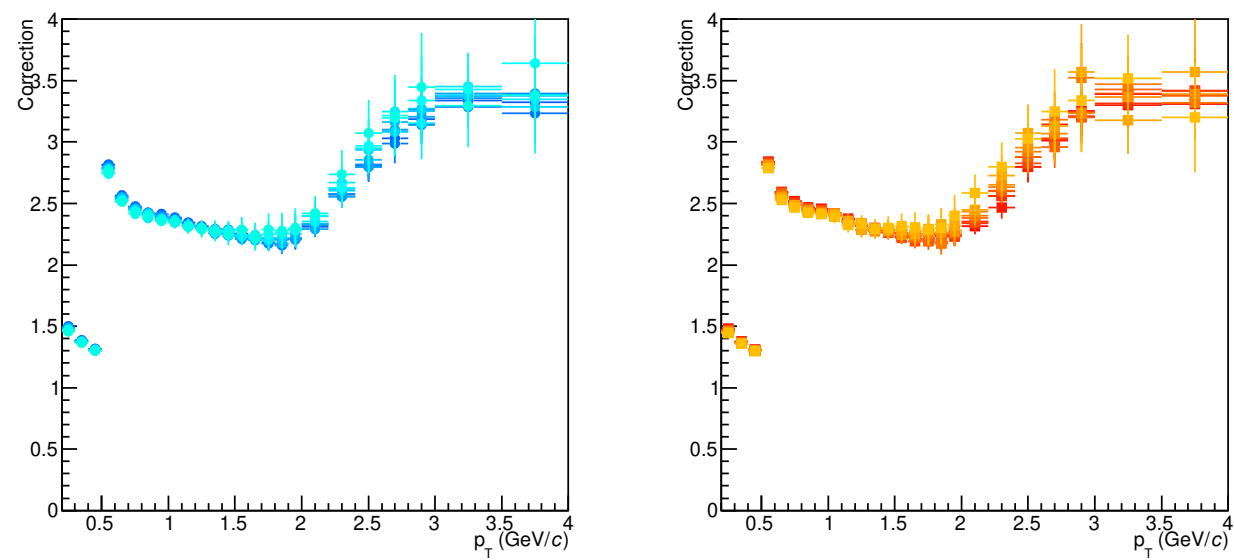

FIGURE 4.11: Final correction factor as a function of transverse momentum for positive (blue) and negative (orange) pions

The same aforementioned procedure is applied for the rest of the associated per-trigger yields, namely $\mathrm{C}_{(-,+)}, \mathrm{C}_{(+,+)}, \mathrm{C}_{(-,-)}$and the balance function is calculated as

$$
B(\Delta \eta, \Delta \varphi)=\frac{1}{2}\left[C_{(+,-)}+C_{(-,+)}-C_{(+,+)}-C_{(-,-)}\right]
$$

where $1 / 2$ factor is used to normalize the integral of the balance function to unity (see Chapter 2 for more details). If the balance function is measured for identified species as done in this analysis, pseudorapidity $(\eta)$ in the formulas above is replaced by rapidity (y) to account for the difference between these observables for more massive particles.

The plot of Figure 4.12 shows a typical balance function distribution as a function of $\Delta \eta$ and $\Delta \varphi$. These distributions, that will be discussed more in detail in Chapter 5 , are then projected in two dimensions. When projecting in $\Delta \mathrm{y}$ the near side of the two-dimensional distribution is considered $(-\pi / 2 \leq \Delta \varphi<\pi / 2)$ separately from the away side $(\pi / 2 \leq \Delta \varphi<3 \pi / 2)$, while when projecting in $\Delta \varphi$ the entire $\Delta y$ (i.e. $|\Delta y|<1)$ is considered. From these projections, the RMS of the distributions for both $\Delta \mathrm{y}$ and $\Delta \varphi$ is extracted for the measurement of the balance function width, the first being computed in the entire range of $\Delta y$ 
and denoted as $\sigma_{\Delta y}$ and the second one only in the near side of the distribution ( $\sigma_{\Delta \varphi}$ in the rest of the thesis). An example of balance function projections in $\Delta y$ and $\Delta \varphi$ for pions for 0-5\% multiplicity class is shown in Figure 4.13. A detailed discussion about the structures and the shapes of these projections is reserved for Chapter 5. In addition, the integral of the balance function is computed for the measurement of the balance function yield. This is done by integrating the projection in $\Delta \varphi$ in three different ranges: the entire range, near-side range and away-side range that provide total yield $\mathrm{Y}_{\mathrm{B}_{\text {total }}}$, near-side $\mathrm{Y}_{\mathrm{B}_{\text {near }}}$ and away-side yield $\mathrm{Y}_{\mathrm{B}_{\text {away }}}$, respectively.

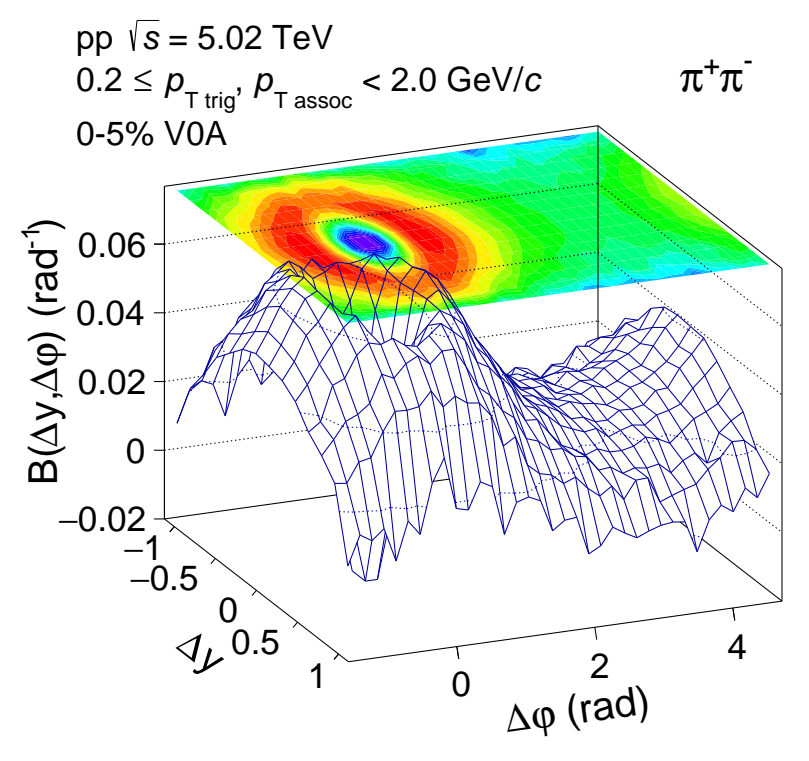

FiguRe 4.12: Two-dimensional balance function distribution for pions for 0$5 \%$ multiplicity class in pp collisions at $\sqrt{\mathrm{s}}=5.02 \mathrm{TeV}$
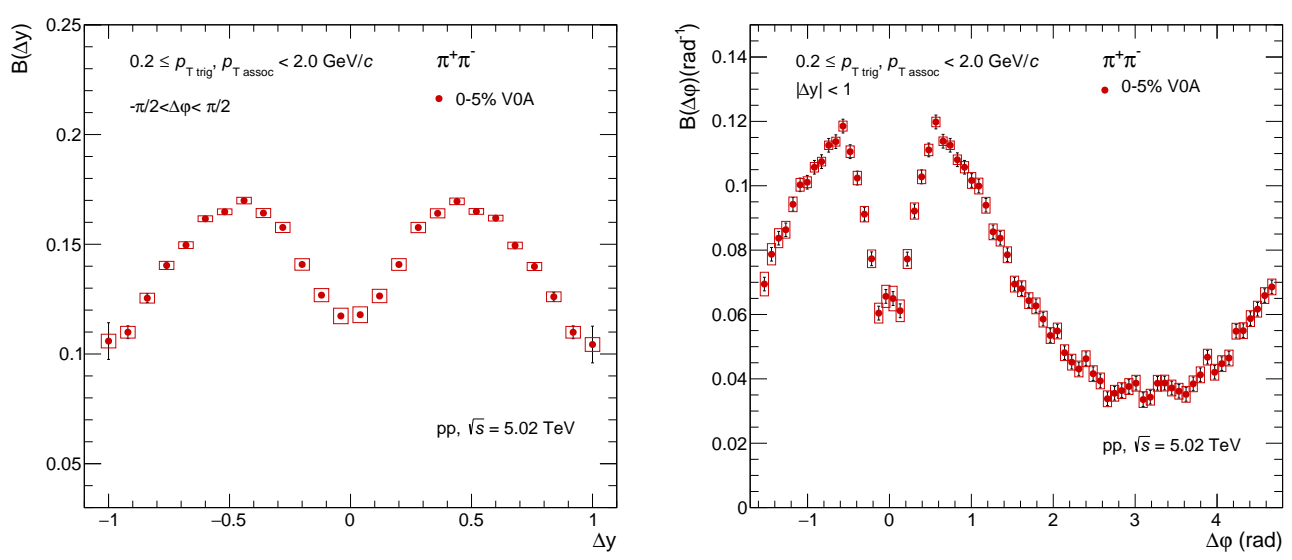

FigURE 4.13: Balance function projection in $\Delta y$ (left) and $\Delta \varphi$ (right) for pions for $0-5 \%$ multiplicity class in pp collisions at $\sqrt{\mathrm{s}}=5.02 \mathrm{TeV}$ 
The statistical uncertainties on the balance function width and the yield are computed using a sampling technique instead of taking the uncertainty calculated by ROOT that assumes a normal distribution. In this technique 10000 new distributions are randomly produced for every bin of the balance function projection both in $\Delta \mathrm{y}$ and $\Delta \varphi$ sampled from a gaussian distribution with a mean being the value of this particular bin and $\sigma$ being the statistical uncertainty of that bin. For each of these new generated projections a new RMS and a yield are computed. The final statical error on the width and the yield of the balance function is estimated as the RMS over all these new 10000 values.

\subsection{Monte Carlo closure test}

This section summarises the results of what is usually referred to as the Monte Carlo closure test that was used to check if the correction factors for detector acceptance, efficiencies and contamination described in Sections 4.3, 4.1.3 and 4.2 were properly applied in the presented analysis.

As a fist step in this test, a Monte Carlo generator is used to generate a sample of primary particles and mimic an outcome of a real event. This is followed by the propagation of the generated particles through the simulated detector setup (referred to as reconstructed Monte Carlo) similar to the one used in the measurement to recreate the same detector effects such as the limited acceptance and inefficiencies that can affect the final results at the level of a real data analysis. In the data analysis, the correction factors are used to correct for such effects. Therefore, applying the same corrections in the reconstructed Monte Carlo should bring the relevant results to an agreement with the same results for the initial generated sample if the correction procedure is proper and if all factors are properly applied.

In this analysis, the closure test was performed using the PYTHIA8 Monte Carlo generator by comparing the results obtained at the generated level and the reconstructed level for each analyzed particle type. The results were computed for $0-100 \%$ multiplicity for pions in $0.2-2 \mathrm{GeV} / \mathrm{c}$ and for protons in $0.5-2 \mathrm{GeV} / \mathrm{c}$ transverse momentum range.

Figure 4.14 shows the comparison of the balance function projections for the generator and the reconstructed Monte Carlo level for pions. The ratio of the generated over reconstructed results can be seen in Figure 4.15 and shows a difference of $0.1 \%$ for the projections in $\Delta y$ obtained as a fit value. For the projections in $\Delta \varphi$ the differences are of the order of $0.8 \%$. Figure 4.16 shows the same comparison for protons while the ratio is presented in Figure 4.17. It 
can be seen that the difference in this case is around $0.4 \%$ for $\Delta y$ projection and around $2 \%$ for $\Delta \varphi$ projection. For both studied particle types (pions and protons) the Monte Carlo closure test closes with the difference being not higher than $2 \%$ that confirms the compatibility between the results at generated and reconstructed levels, hence the proper application of correction factors. The remaining difference is included in the estimation of the systematic uncertainty described in Chapter 5.
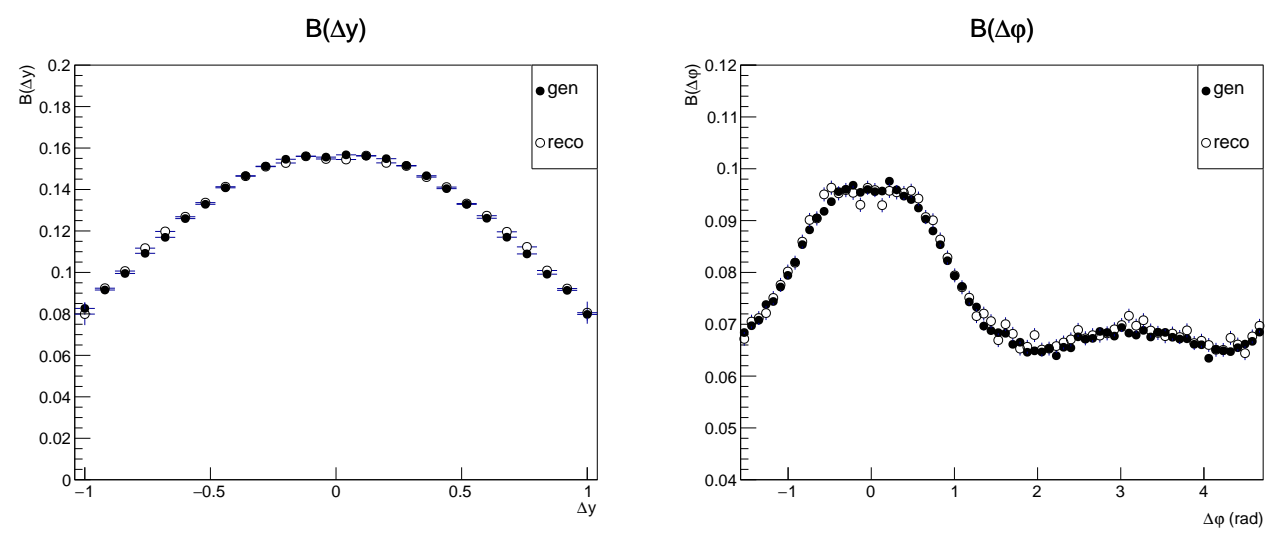

FiguRE 4.14: MC closure test for pions: balance function projections in $\Delta \mathrm{y}$ (left) and $\Delta \varphi$ (right)
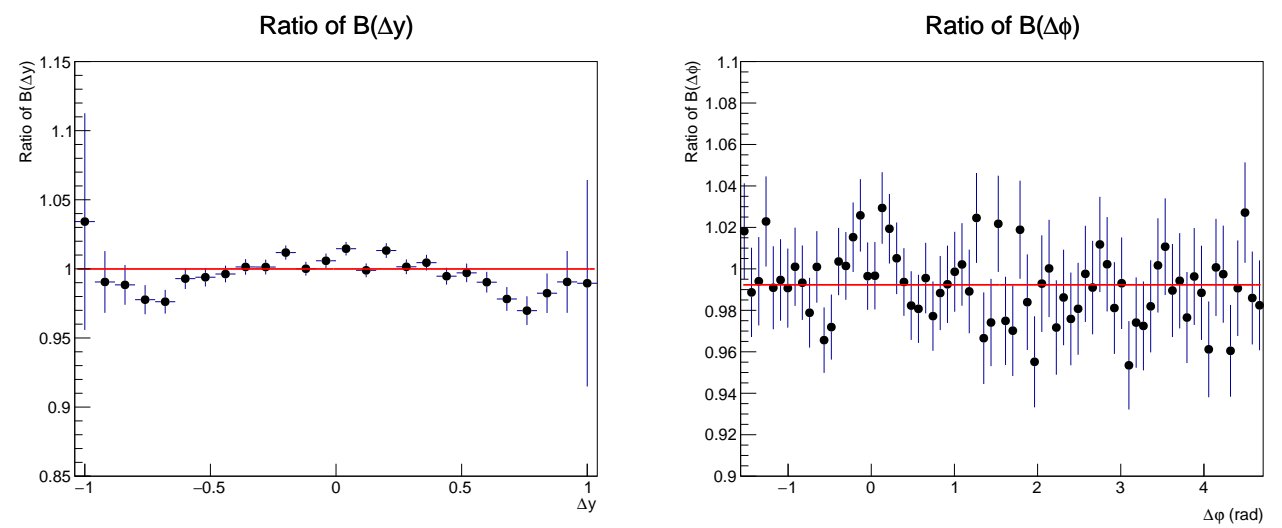

FiguRE 4.15: MC closure test for pions: ratio of the balance function projections at the generator level over reconstructed level in $\Delta \mathrm{y}$ (left) and $\Delta \varphi$ (right) 

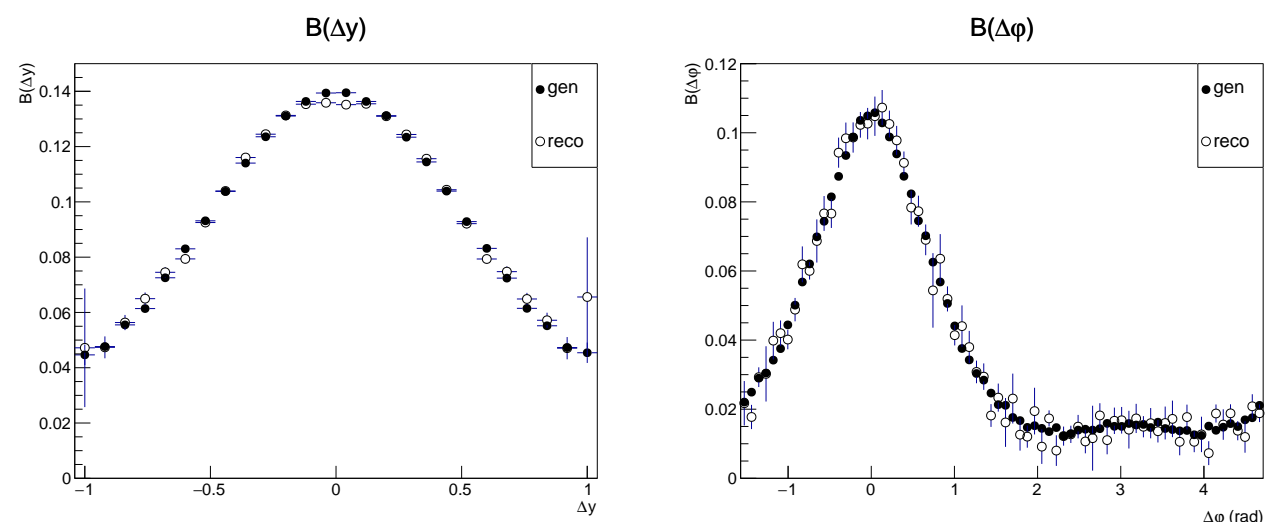

FigURE 4.16: MC closure test for protons: balance function projections in $\Delta \mathrm{y}$ (left) and $\Delta \varphi$ (right)
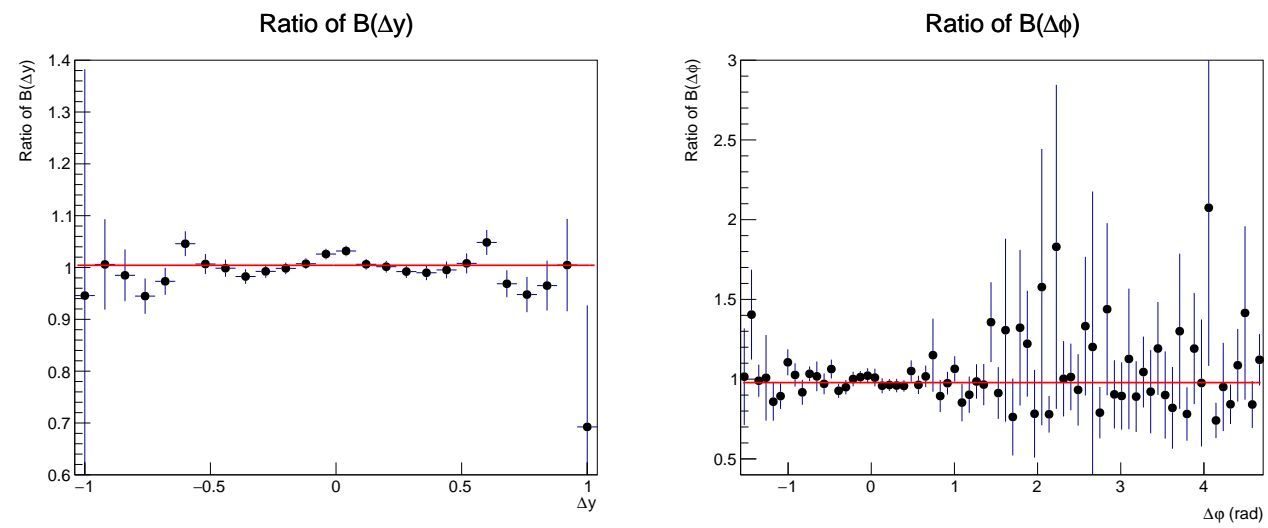

FIGURE 4.17: MC closure test for protons: ratio of the balance function projections at the generator level over reconstructed level in $\Delta \mathrm{y}$ (left) and $\Delta \varphi$ (right) 


\section{Chapter 5}

\section{Results}

This section presents the results of the balance function measurement in pp collisions at $\sqrt{\mathrm{s}}=5.02 \mathrm{TeV}$. At first, the results for charged particles are shown in Section 5.1.1. For each point in the presented balance function projections, widths and yields not only statistical but also systematic uncertainties are indicated. The procedure of the systematic uncertainty calculation and the considered sources is explained in Section 5.1.2.

Next, the results of the two-dimensional balance functions for identified particle species, namely pions, kaons and protons are discussed in Section 5.2.1. Due to the contribution from resonances decaying into $\pi^{+} \pi^{-}$pairs in the balance function of pions that is not easy to remove in the data analysis, Monte Carlo simulation study using the PYTHIA8 event generator was performed instead to estimate possible effects. Similar study was done for kaons because of a significant part of charge correlations coming from the $\phi$ meson decay into $\mathrm{K}^{+} \mathrm{K}^{-}$ pairs. The results for both cases are described in Section 5.2.2. Since the $\phi$ contribution significantly affects the shape of the kaon balance function, the final results for kaons should be reported with the $\phi$ meson being excluded in the data analysis. Therefore, the results of the balance function projections, the width and the yield are reported only for pions and protons in Sections 5.2.3 and 5.2.4.

Finally, Section 5.2.5 presents the comparison of the measured pion and proton balance function distributions to the corresponding predictions from the PYTHIA8 model, while Section 5.3 - the comparison to similar results in p- $\mathrm{Pb}$ collisions at the same energy. 


\subsection{Balance function of charged particles in pp colli- sions at $\sqrt{\mathrm{s}}=5.02 \mathrm{TeV}$}

\subsubsection{Two-dimensional balance function distributions}

Figure 5.1 presents the results of the two-dimensional balance function distributions for 8 multiplicity classes from 0-5\% (highest multiplicity) to $70-80 \%$ (lowest multiplicity) for charged particles measured in pp collisions at $\sqrt{\mathrm{s}}=5.02 \mathrm{TeV}$. A distinct depletion around $(\Delta \eta, \Delta \varphi)=(0,0)$ is observed that becomes broader and more pronounced with decreasing multiplicity. Similar structure was observed in the previous measurements at $\sqrt{\mathrm{s}}=7 \mathrm{TeV}$, where it was attributed to quantum statistics correlations of identical bosons and Coulomb effects [111]. To test this hypothesis, a requirement on the minimum transverse momentum difference $\Delta \mathrm{p}_{\mathrm{T}}$ between two particles of a pair was varied from $0 \mathrm{GeV} / \mathrm{c}$ to $0.2 \mathrm{GeV} / \mathrm{c}$. The choice of such values was driven by the fact that the bulk of short-range correlations, such as the ones originating from quantum statistics, is expected to have $\Delta \mathrm{p}_{\mathrm{T}}<0.1 \mathrm{GeV} / \mathrm{c}[130]$. As a result, for the increased value of $\Delta \mathrm{p}_{\mathrm{T}}$ the depletion was less pronounced and vanished for $\Delta \mathrm{p}_{\mathrm{T}}=0.2 \mathrm{GeV} / \mathrm{c}$. Moreover, another test was performed by increasing the lower transverse momentum threshold for both the trigger and the associated particle to $\mathrm{p}_{\mathrm{T}}>0.5 \mathrm{GeV} / \mathrm{c}$ that also lead to the disappearance of the structure. These observations are therefore both in agreement with the hypothesis.

Since the projections of the two-dimensional distributions as well as the width and the yields presented later in the text are shown including the systematic uncertainties, next section explains the procedure and sources considered for the systematic uncertainty evaluation. 

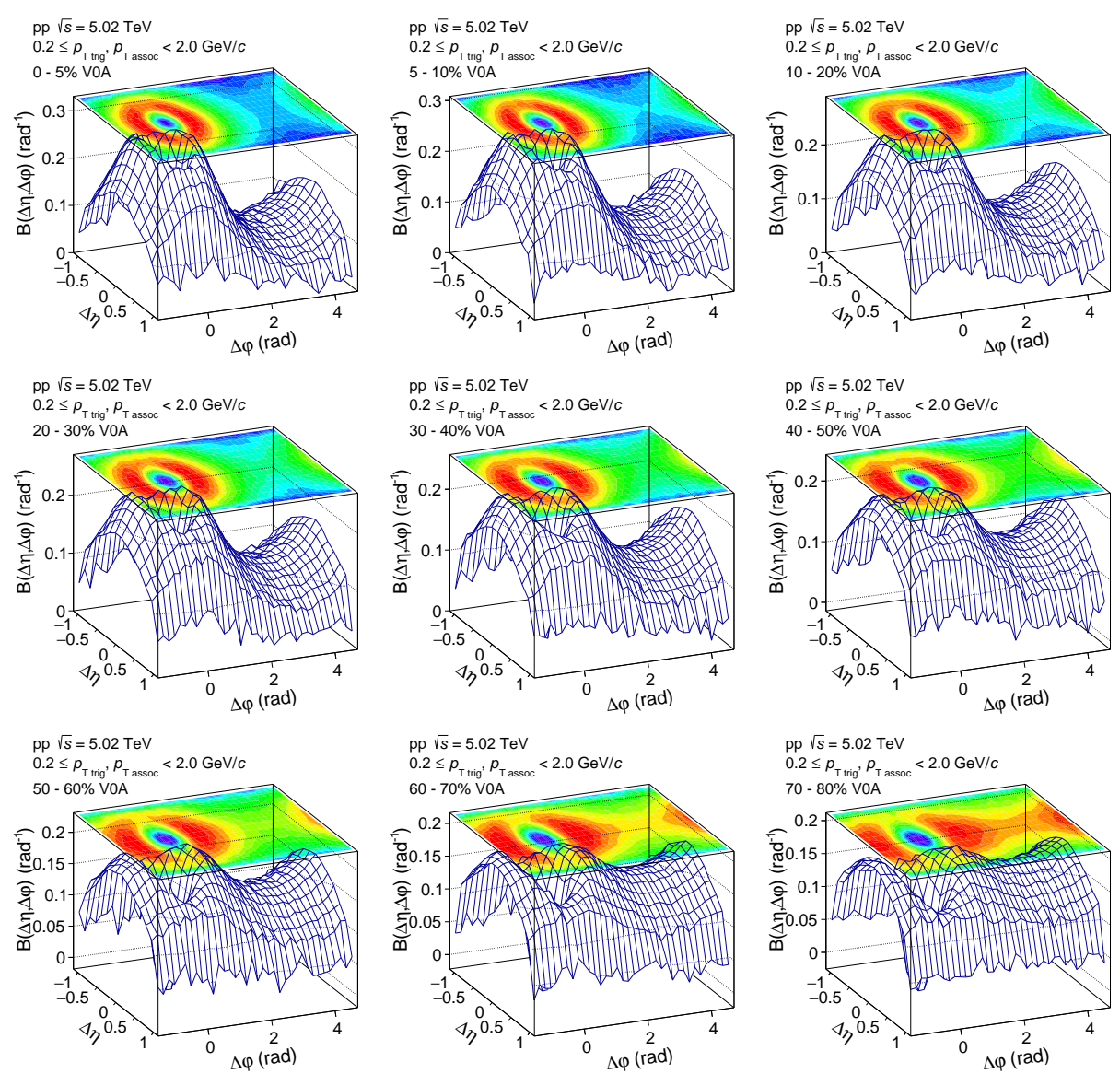

FiguRE 5.1: Two-dimensional balance function of charged particles in pp collisions at $\sqrt{\mathrm{s}}=5.02 \mathrm{TeV}$ as a function of multiplicity class

\subsubsection{Systematic uncertainties}

The systematic uncertainties for the balance function projections, width and yields presented in the following sections were calculated by varying a number of analysis parameters, resulting into several trials which can give rise to systematic biases. For each of the sources the systematic bias was computed by calculating the difference between the default and the trial measurement and evaluating whether the difference was more than $1 \sigma$ away from 0 , where $\sigma$ is the relevant uncertainty of the difference considering the degree of correlation between the two results. If the majority of the points satisfied this requirement, the source was considered statistically significant and was taken into account in the calculation of the systematic uncertainty.

The difference was then symmetrized and smoothened by fitting it with a function depending on the result and assigning the systematic uncertainty for every bin in $\Delta y$ and $\sigma_{\Delta \varphi}$ as half of the fit value. In case of few variations, the one giving the maximum difference was chosen. The statistical uncertainty on the 
difference was calculated assuming that the trial and default measurements were fully correlated with exception of the data sample variation, where they were considered uncorrelated. The final central value was calculated as the average among all trials, while the final systematic uncertainty was calculated adding all contributing values in quadrature. The maximum contribution to the systematic uncertainty from every source for each multiplicity class is summarised in Section 5.1.2.3.

\subsubsection{Procedure}

To demonstrate the procedure of estimating the systematic uncertainty, few examples are shown below. Figure 5.2 presents the pion balance function width $\sigma_{\Delta y}$ for the default set of track selection criteria used in the analysis (so called filter bit 96) and two variations of this default configuration (filter bit 1 and 16). The difference between the default measurement and the variations is presented in Figure 5.3. A systematic trend can be observed since all the points lie more than $1 \sigma$ away from 0 . The maximum deviation given by filter bit 1 is fitted with a constant function from where the same systematic uncertainty is estimated for every bin.

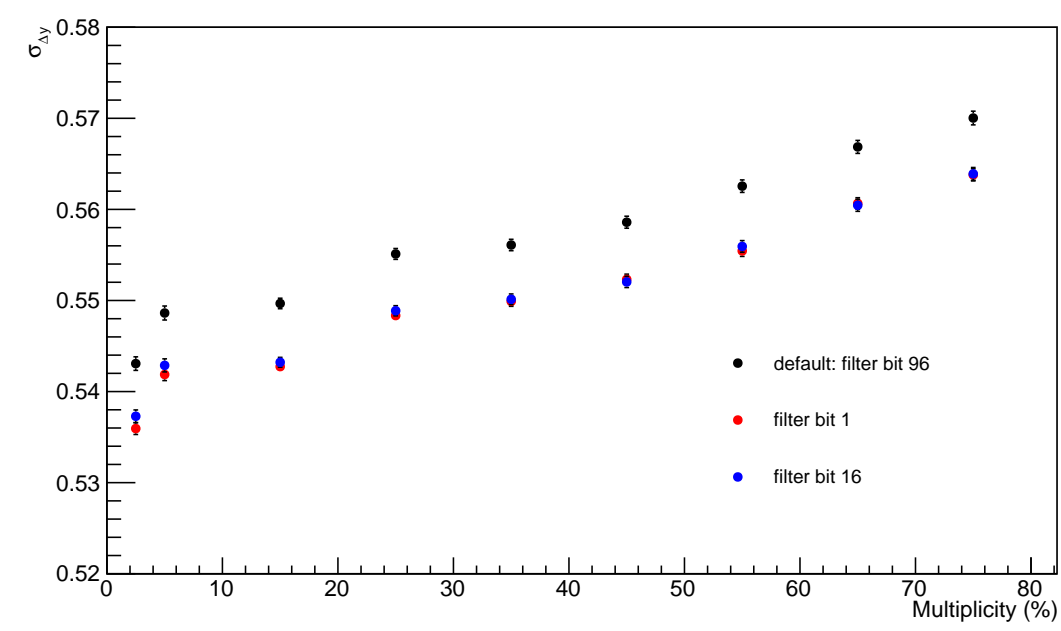

FiguRE 5.2: Balance function width $\sigma_{\Delta y}$ of pions as a function of multiplicity class for the default set of track selection criteria (filter bit 96) and two variations (filter bit 1 and filter bit 16) 


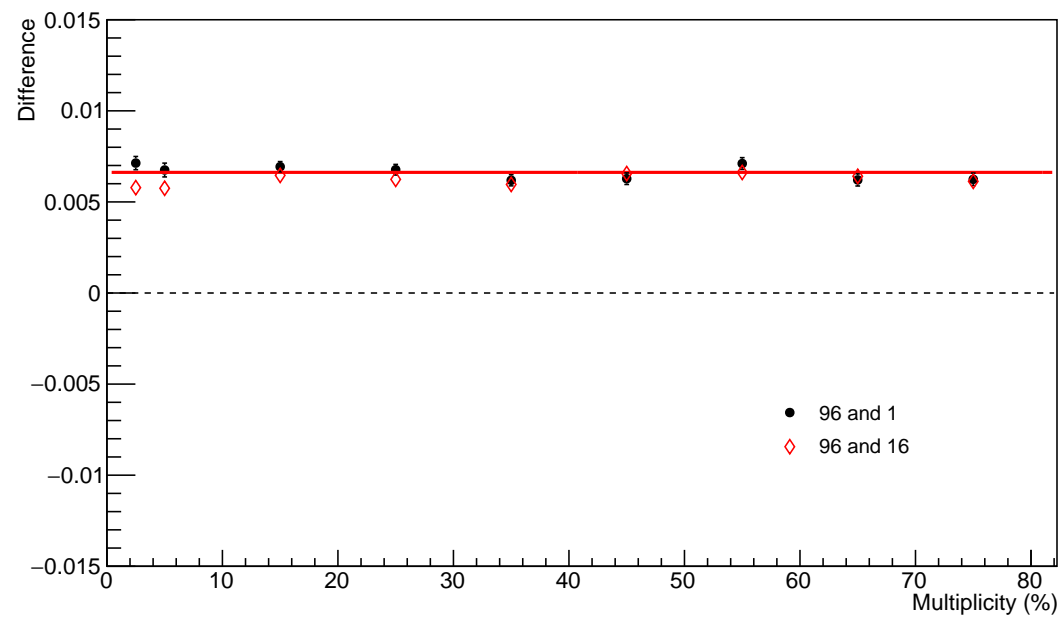

FIGURE 5.3: Difference between the balance function width $\sigma_{\Delta y}$ of pions as a function of multiplicity class for default set of track selection criteria (filter bit 96) and two variations (filter bits 1 and 16)

Figure 5.4 shows the pion balance function projections in $\Delta y$ for $0-5 \%$ multiplicity class for the same default measurement and variations, while the difference between them is presented in Figure 5.5. In this case the fit is multiplicitydependent and leads to a different systematic uncertainty assigned for every bin.

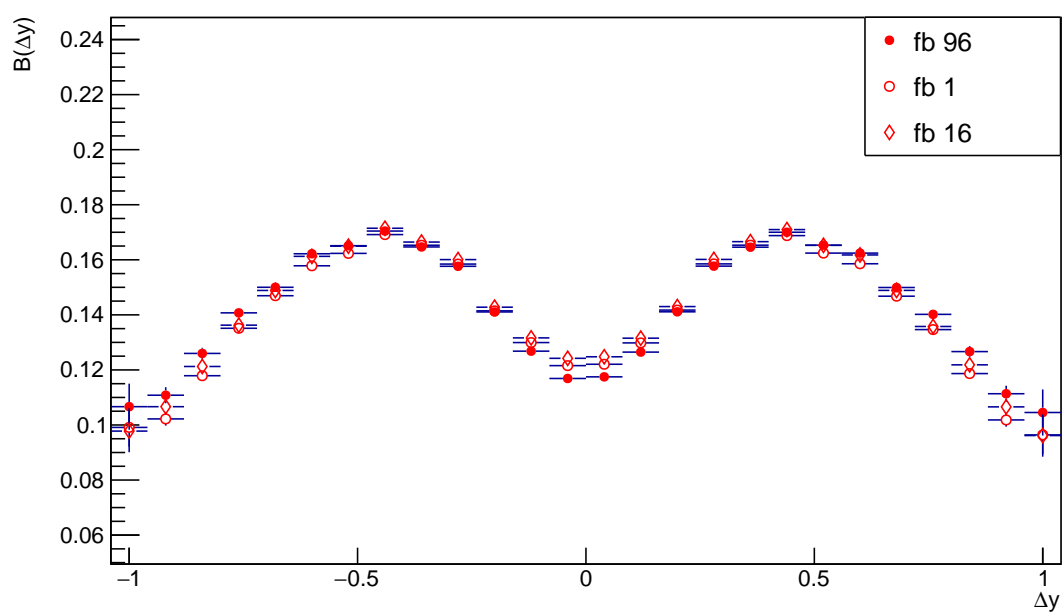

Figure 5.4: Balance function projection of pions in $\Delta y$ for $0-5 \%$ multiplicity class for the default set of track selection criteria (filter bit 96) and two variations (filter bit 1 and filter bit 16) 


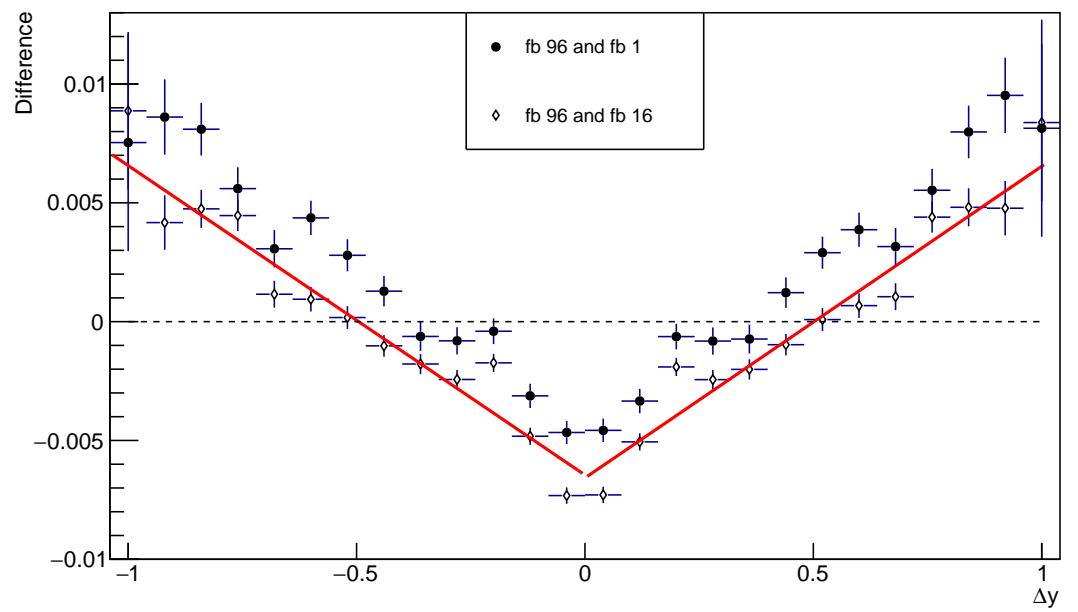

FIGURE 5.5: Difference between balance function projection of pions in $\Delta y$ for 0-5\% multiplicity class for the default set of track selection criteria (filter bit 96) and two variations (filter bit 1 and filter bit 16)

\subsubsection{Sources}

The following sources were considered for the estimation of the systematic uncertainty:

\section{- Data sample}

To evaluate any potential bias introduced by the selection of the data sample, a different one called "wSDD" from now on in the text was also analyzed separately. This sample provides all events with the SDD detector in the readout as well as the SDD information included at the level of the track reconstruction.

As an example, Figure 5.6 presents the comparison of the trial and the default measurements for the balance function width in $\Delta \eta$ for the two data samples analyzed. Figure 5.7 shows the difference between both measurements. It can be seen that most of the points are compatible with 0 within their uncertainties. The fit gives a difference of $-0.0005 \pm 0.0004$ that is compatible with 0 . Therefore, this source does not contribute to the systematic uncertainty. 


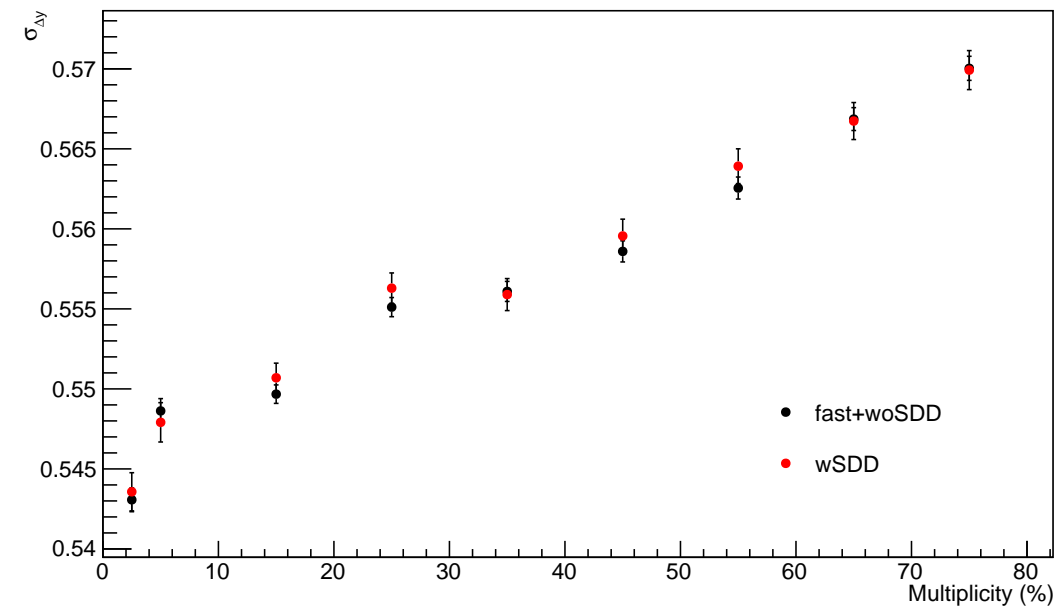

FiguRE 5.6: Balance function width $\sigma_{\Delta y}$ of pions as a function of multiplicity class for the default data sample (fast+woSDD) and the variation (wSDD)

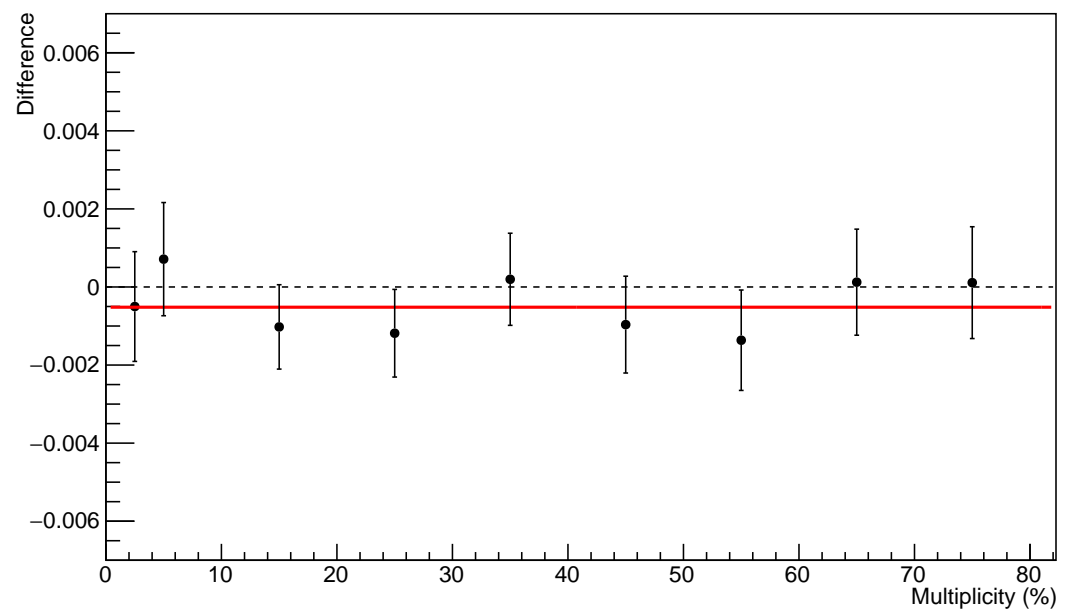

FiguRE 5.7: Difference between the balance function width $\sigma_{\Delta y}$ of pions as a function of multiplicity class for the default data sample (fast+woSDD) and the variation (wSDD)

\section{- Track selection criteria}

The set of default track selection criteria described in Section 4.1.3 (referred later in the results as filter bit 96) was varied by changing, for instance, a requirement on the available detector information. In one of the variations (referred later in the results as filter bit 1) the tracks only with TPC information were used. In another variation (referred later in the results as filter bit 16) a requirement on $\mathrm{DCA}_{\mathrm{xy}}$ value of the track was changed among other criteria. 
As an example, Figure 5.8 presents the comparison of the trial and the default measurements for the balance function width in $\Delta \eta$ for the track selection criteria variation. Figure 5.9 presents the difference between the default measurement and the variations. The systematic trend is observed - all the points lie more than $1 \sigma$ away from 0 . The fit to the maximum deviation given by filter bit 1 estimates the difference as $0.0066 \pm 0.0002$, or as $1.2 \%$ given by the fit to the ratio of the results. The maximum contribution of this source to the systematic uncertainty is then around $0.6 \%$ for every multiplicity bin.

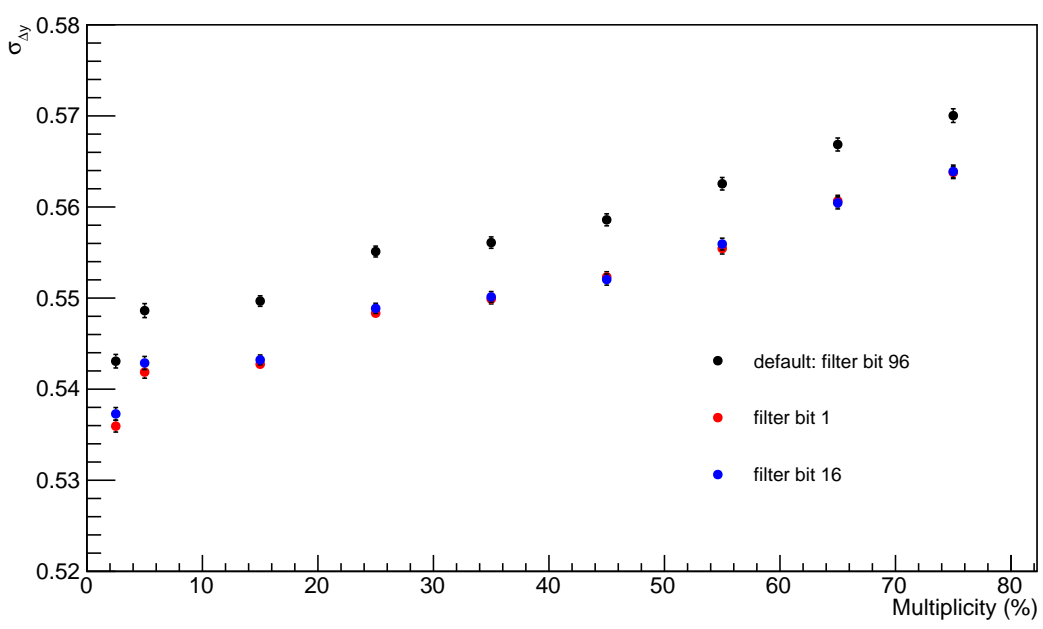

FIGURE 5.8: Balance function width $\sigma_{\Delta y}$ of pions as a function of multiplicity class for the default track selection criteria set (denoted as filter bit 96) and two variations (denoted as filter bits 1 and 16)

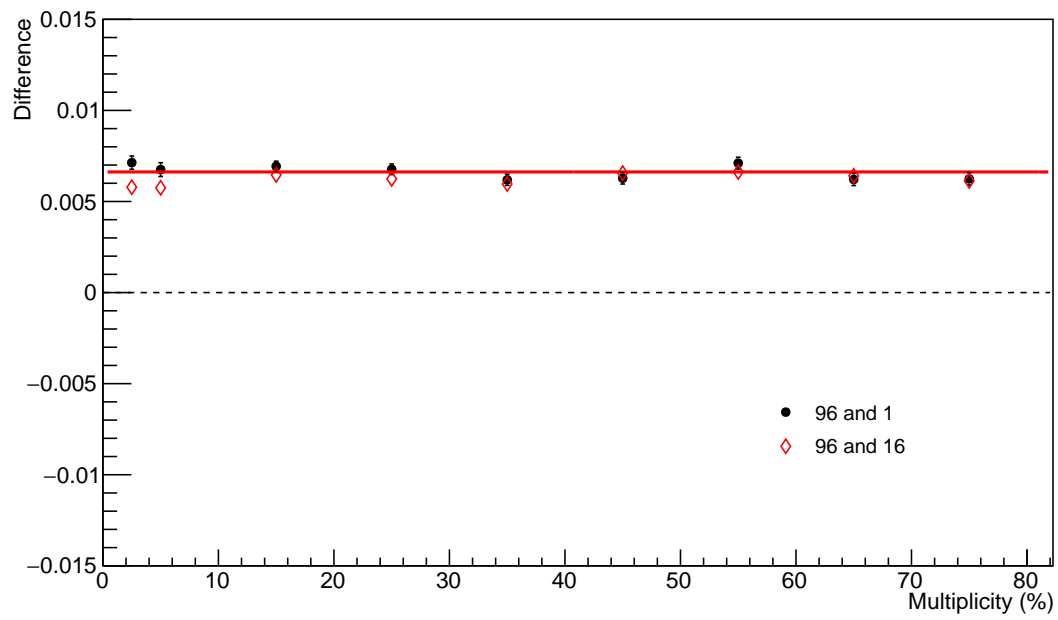

Figure 5.9: Difference between the balance function width $\sigma_{\Delta y}$ of pions as a function of multiplicity class for default set of track selection criteria (filter bit 96) and two variations (filter bits 1 and 16) 


\section{- $\Delta \varphi^{*}$ cut}

The default measurement was done by using the requirement on $\Delta \varphi^{*}$ between two tracks described in Section 4.1.3 to be 0.02, while two variations were $\Delta \varphi^{*}=0.01$ and $\Delta \varphi^{*}=0.03$.

As an example, Figure 5.10 presents the comparison of the trial and the default measurements for the balance function width in $\Delta \eta$ for $\Delta \varphi^{*}$ cut variation. The difference between the default measurement and the variations is presented in Figure 5.11. The larger deviation from the default points with the systematic trend is observed in case of $\Delta \varphi^{*}=0.01$ cut. The corresponding fit value is $0.00027 \pm 0.00003$, or $0.05 \%$ as given by the fit to the ratio of the results. Therefore, the maximum contribution of this source to the systematic uncertainty is about $0.025 \%$ for every multiplicity bin.

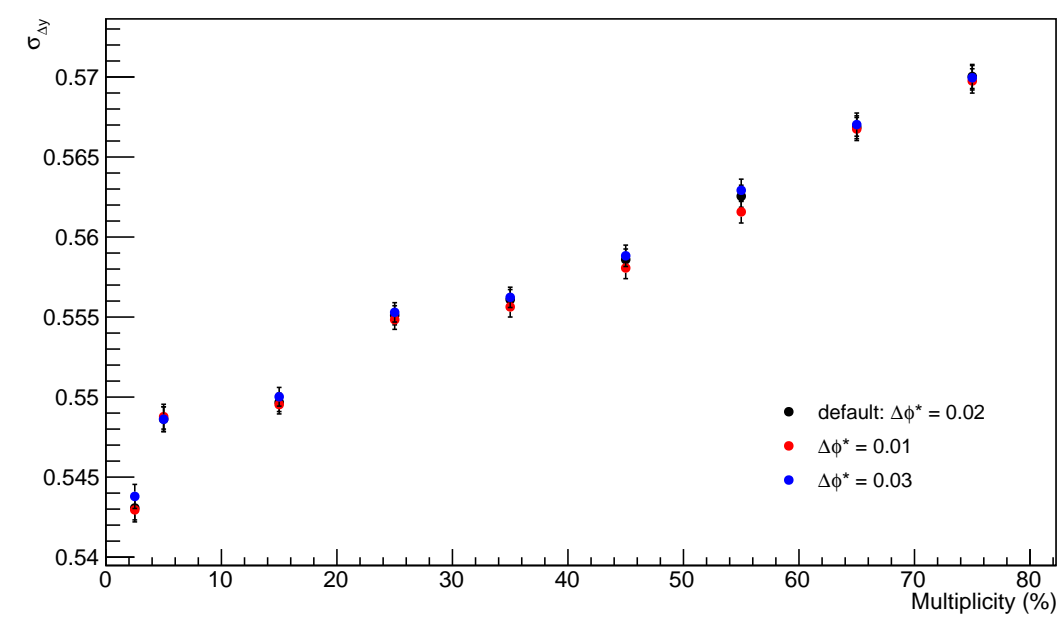

FIGURE 5.10: Balance function width $\sigma_{\Delta y}$ of pions as a function of multiplicity class for the default $\Delta \varphi^{*}$ cut (0.02) and the variations (0.01 and 0.03$)$ 


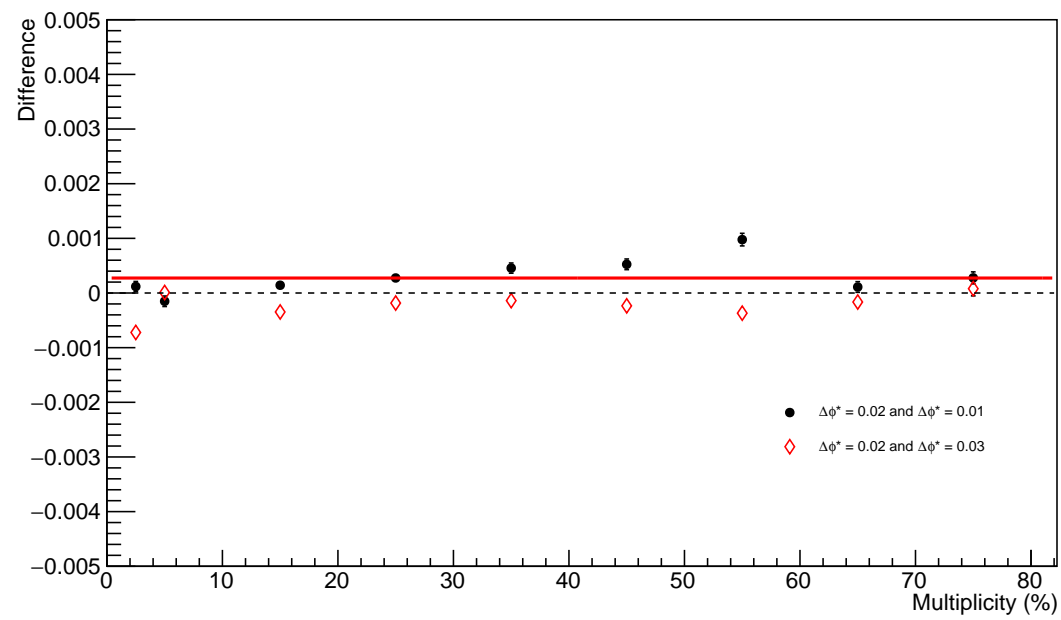

FiguRE 5.11: Difference between the balance function width $\sigma_{\Delta y}$ of pions as a function of multiplicity class for the default $\Delta \varphi^{*}$ cut (0.02) and the variations (0.01 and 0.03)

\section{- $\mathrm{V}_{\mathrm{z}}$}

As mentioned in Section 4.1.2, only events with the z position of the primary vertex within $10 \mathrm{~cm}$ from the nominal interaction point were selected for the analysis. This requirement was varied by using 7 and $3 \mathrm{~cm}$ requirement instead.

As an example, Figure 5.12 presents the comparison of the trial and the default measurements for the balance function width in $\Delta \eta$ for the $V_{z}$ variation. Figure 5.13 shows the difference between both measurements. While the difference between the results for $V_{z}$ of $[-10,10]$ and $V_{z}$ of $[-3,3]$ is compatible with 0 , a slight deviation is observed in case of the difference between the results for $\mathrm{V}_{\mathrm{z}}$ of $[-10,10]$ and $\mathrm{V}_{\mathrm{z}}$ of $[-7,7]$ where 4 points lie more than $1 \sigma$ from 0 . The fit to the latter result gives the difference of $-0.00023 \pm 0.00025$ which is compatible with 0 , therefore no systematic uncertainty is assigned for this source. 


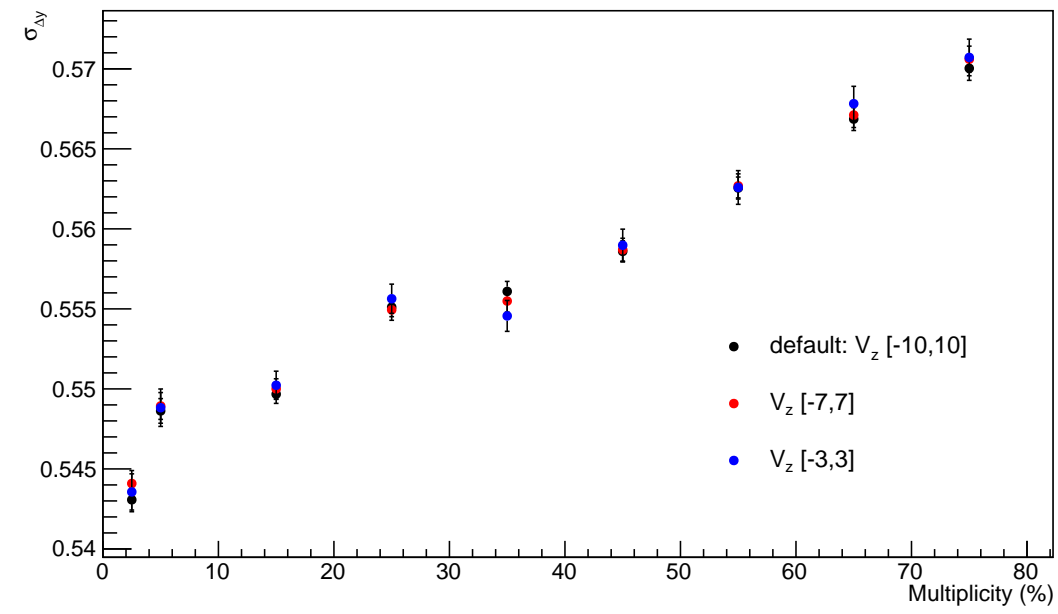

Figure 5.12: Balance function width $\sigma_{\Delta y}$ of pions as a function of multiplicity class for the default $\mathrm{V}_{\mathrm{z}}([-10,10])$ and the variations $([-7,7]$ and $[-3,3])$

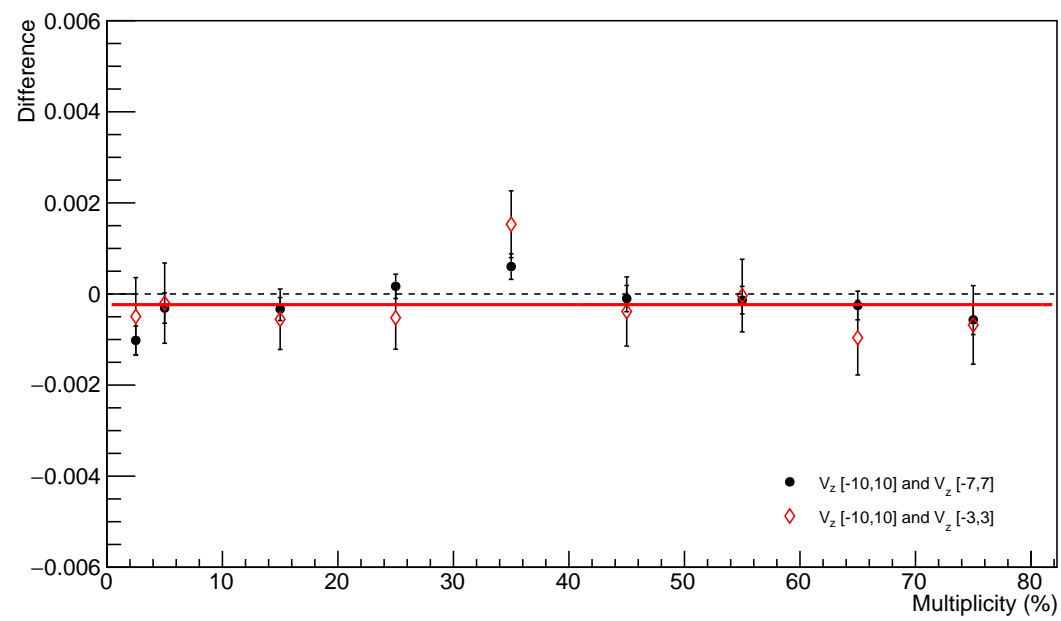

Figure 5.13: Difference between the balance function width $\sigma_{\Delta y}$ of pions as a function of multiplicity class for the default $\mathrm{V}_{\mathrm{z}}([-10,10])$ and the variations $([-7,7]$ and $[-3,3])$

\section{- Pile-up}

The parameters of the default pile-up removal algorithm described in Section 4.1 .2 were varied by, first, changing the default selection algorithm that uses tracks to identify the primary vertex (track vertexer) to the one using only SPD clusters (SPD vertexer). In a second variation, the default number of the minimum number of contributors to the pile-up candidate vertices (SPD clusters) used in the SPD vertexer was changed from 5 to 3 to increase the probability of the rejection. 
The fraction of events tagged as a pile-up was quite similar for all three cases: in case of the track vertexer cut it was $3 \%$ of the total amount, in case of SPD vertexer cut with 5 contributors - $2 \%$ and with 3 contributors $-3 \%$.

As an example, Figure 5.14 presents the comparison of the trial and the default measurements for the balance function width in $\Delta \eta$ for the pile-up removal conditions variation. The difference between the default measurement and the variations is presented in Figure 5.15. Both results are compatible and don't show any significant systematic trend. The fit to the difference with respect to the variation estimates it to be compatible with 0 . No systematic uncertainty is assigned for this source.

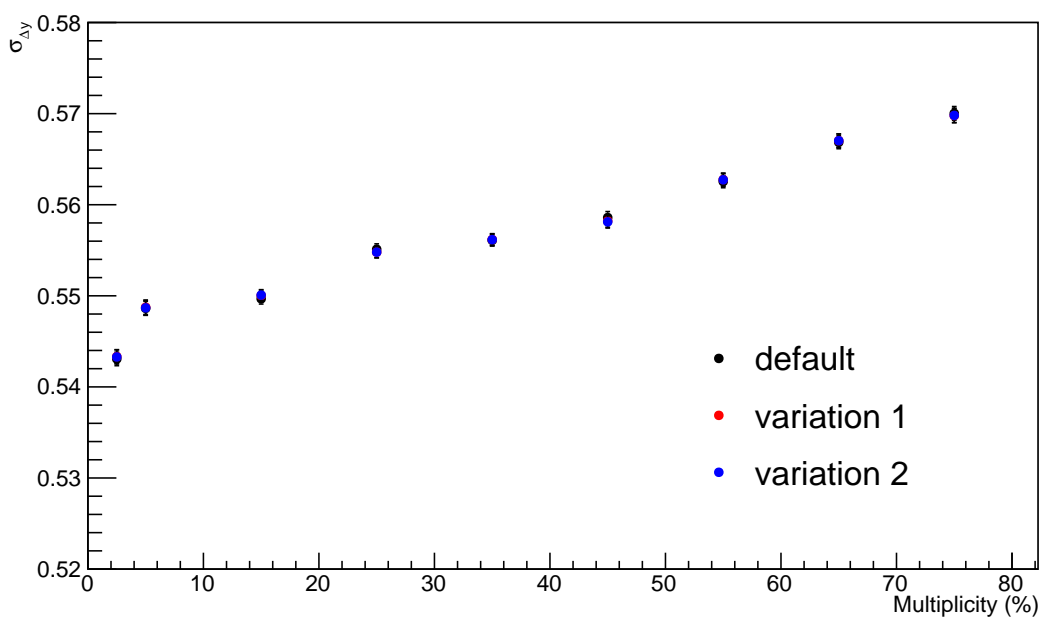

FIGURE 5.14: Balance function width $\sigma_{\Delta y}$ of pions as a function of multiplicity class for the default pile-up cut and the variations 


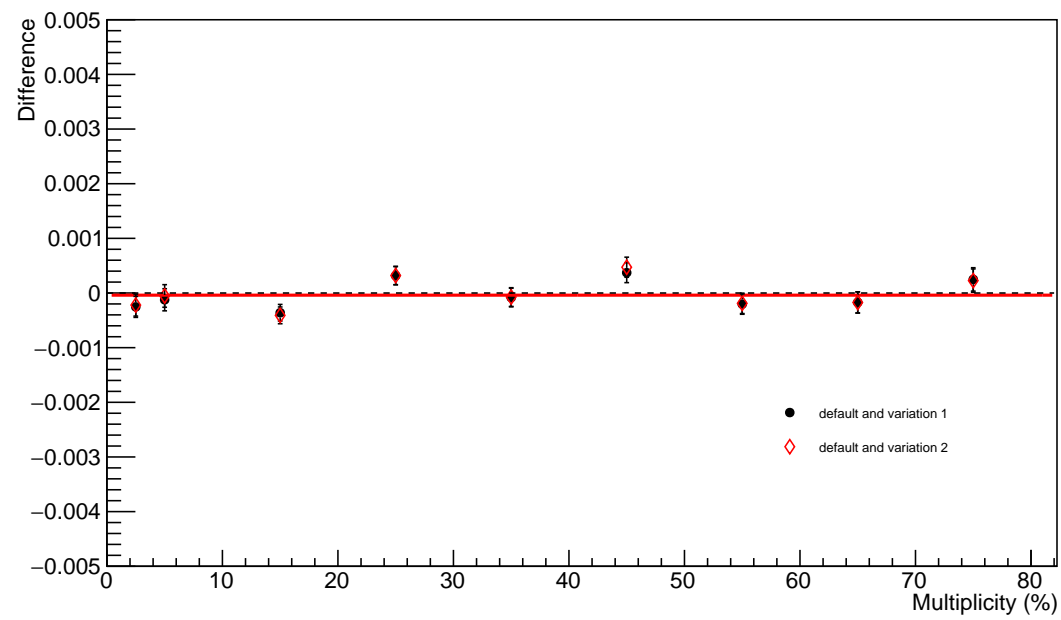

FiguRE 5.15: Difference between the balance function width $\sigma_{\Delta y}$ of pions as a function of multiplicity class for the default pile-up cut and the variations

\section{- Minimum number of TPC clusters}

The default amount of the minimum number of TPC clusters implemented in the default set of track selection criteria and described in more detail in Section 4.1.3 was changed from 50 to 100 for the variation.

As an example, Figure 5.16 presents the comparison of the trial and the default measurements for the balance function width in $\Delta \eta$ for the variation of the number of TPC clusters. Figure 5.17 shows the difference between both measurements. A systematic trend is observed since all the points lie below 0 more than $1 \sigma$ away. The fit estimates the difference as $0.00127 \pm 0.00004$, or as $0.2 \%$ given by the fit to the ratio of the results. The contribution of this source to the systematic uncertainty is $0.1 \%$ for every multiplicity bin. 


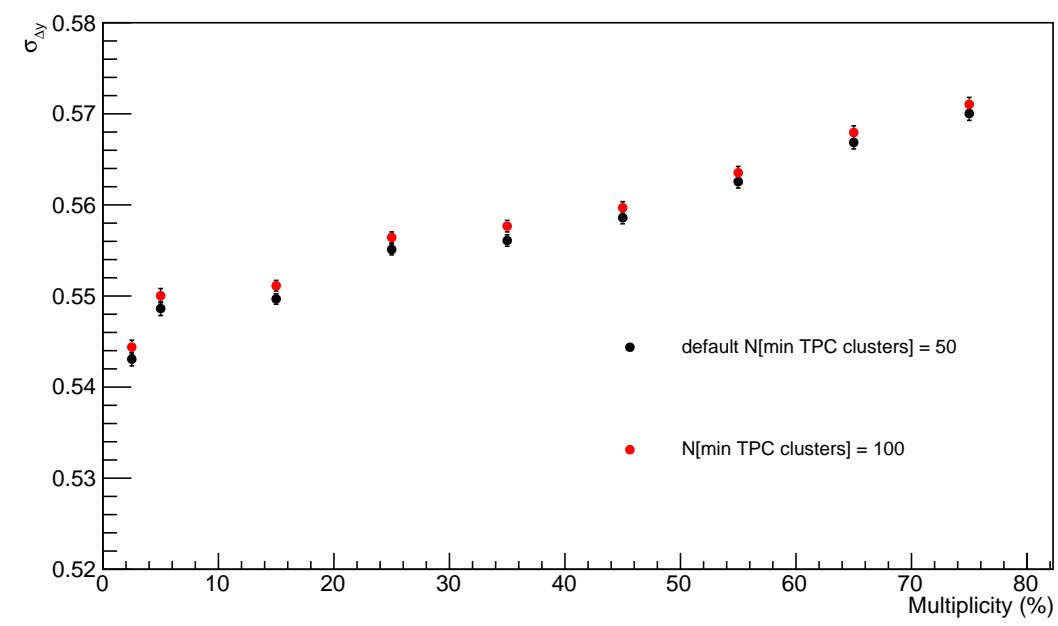

FIGURE 5.16: Balance function width $\sigma_{\Delta y}$ of pions as a function of multiplicity class for the default minimum number of TPC clusters cut (50) and the variation (100)

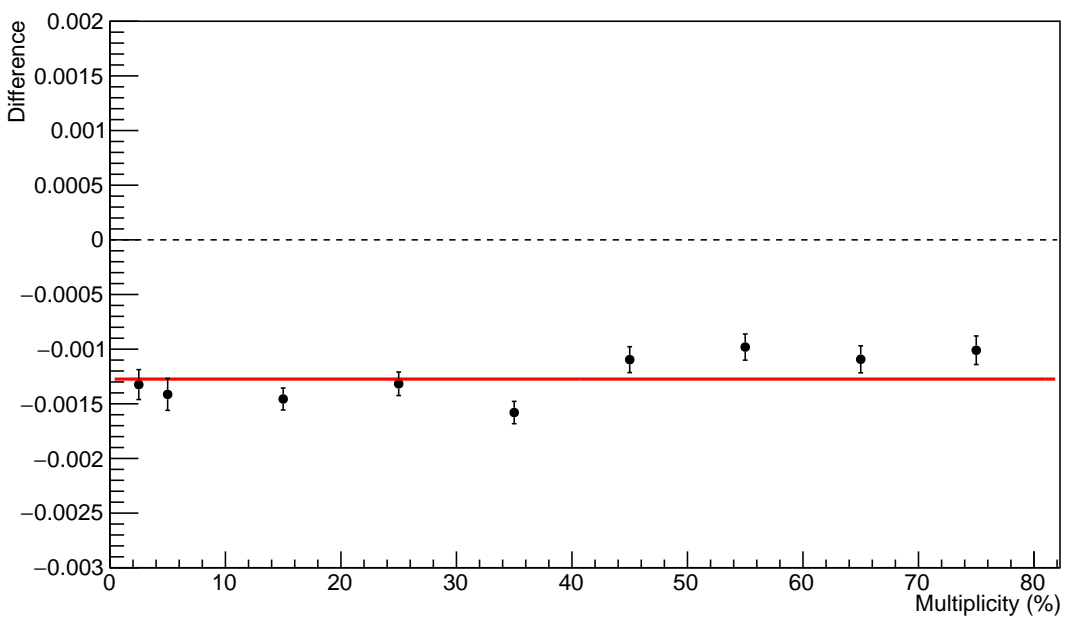

FiguRE 5.17: Difference between the balance function width $\sigma_{\Delta y}$ of pions as a function of multiplicity class for the default minimum number of TPC clusters cut (50) and the variation (100)

\section{- Maximum $\chi^{2}$ per TPC cluster}

The default amount of the maximum $\chi^{2}$ per TPC cluster implemented in the set of track selection criteria and described in more detail in Section 4.1.3 was changed from 4 to 3 for the variation.

As an example, Figure 5.18 presents the comparison of the trial and the default measurements for the balance function width in $\Delta \eta$ for the variation of the maximum $\chi^{2}$ value per TPC cluster. Figure 5.19 shows the difference between both measurements. Also here a systematic trend is observed 
since the majority of the points lie above 0 more than $1 \sigma$ away. The fit estimates the difference as $0.00019 \pm 0.00005$, or as $0.035 \%$ given by the fit to the ratio of the results. The contribution to the systematic uncertainty for this source is then about $0.02 \%$ for every multiplicity bin.

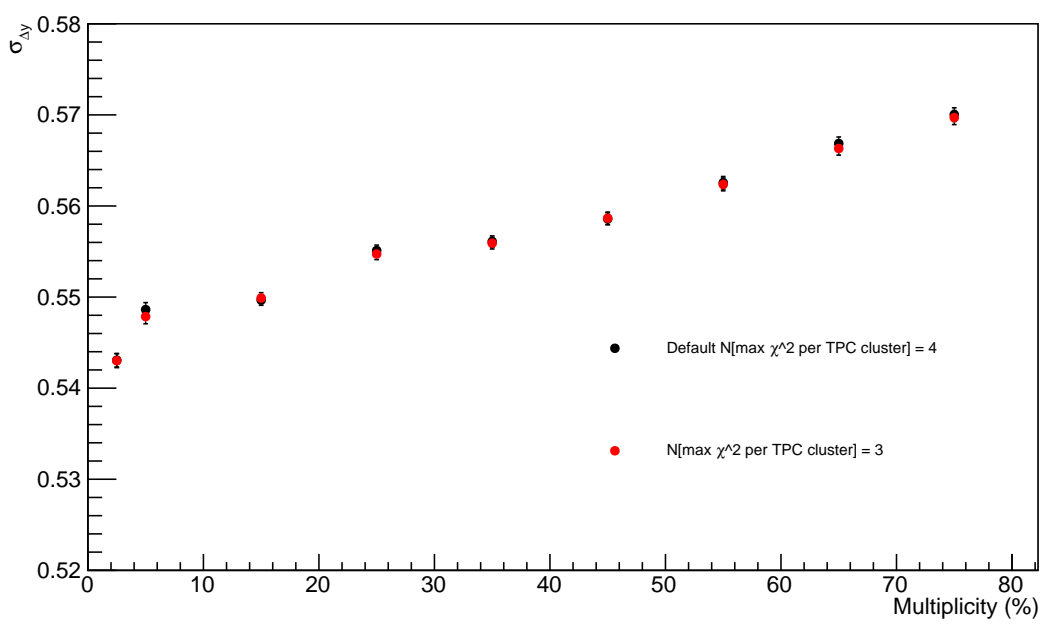

FIGURE 5.18: Balance function width $\sigma_{\Delta \mathrm{y}}$ of pions as a function of multiplicity class for the default maximum $\chi^{2}$ per TPC cluster cut (4) and the variation (3)

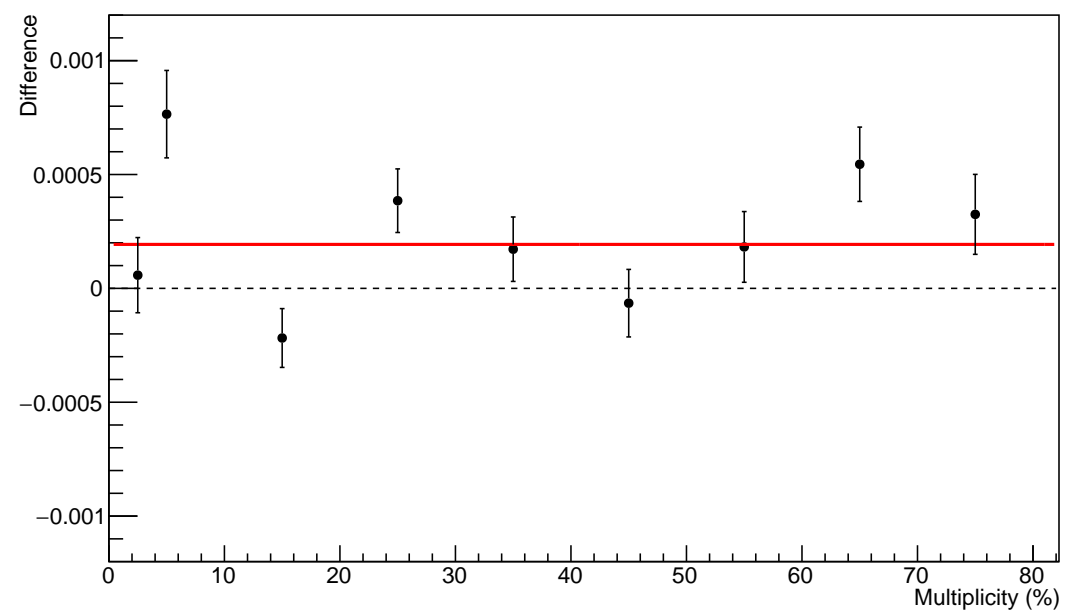

Figure 5.19: Difference between the balance function width $\sigma_{\Delta y}$ of pions as a function of multiplicity class for the default maximum $\chi^{2}$ per TPC cluster cut (4) and the variation (3)

\section{- MC closure}

The difference between the results obtained after analysing the generated sample and the ones obtained analysing the reconstructed output that was still left in Monte Carlo closure test explained in Section 4.4 was included 
in the systematic uncertainty of the balance function projection and the width. Since the closure test was performed in $0-100 \%$ multiplicity class, the resulting systematic uncertainty was assigned as the same for all multiplicity bins.

\section{- PID strategy}

The PID strategy was varied using the Monte Carlo simulation to understand if different selection criteria can bring any significant bias to the purity of the selected particle samples. However, it was found that in the studied low- $\mathrm{p}_{\mathrm{T}}$ part of $0.2-2 \mathrm{GeV} / \mathrm{c}$ the change in the PID selection criteria only changed the PID efficiency, but did not change purities for all particle types. This would mean that the selected particle sample would not be contaminated in a different way by other particle types, so this would not lead to a drastic change in the results. Therefore, the PID strategy variation was not included as a source in the systematic uncertainty estimation in the balance function measurement itself.

Three different PID strategies with no values variations of the default one described in Section 4.2 were used in this study:

$-p_{T}<0.5 \mathrm{GeV} / c:$
$* 1$ variation
\begin{tabular}{|c|c|c|c|}
\hline particle type & $\left|\mathrm{n} \sigma_{\mathrm{TPC}_{\pi}}\right|$ & $\left|\mathrm{n} \sigma_{\mathrm{TPC}_{\mathrm{K}}}\right|$ & $\left|\mathrm{n} \sigma_{\mathrm{TPC}_{\mathrm{p}}}\right|$ \\
\hline pions & $<1.5$ & $>3$ & $>3$ \\
\hline kaons & $>3$ & $<1.5$ & $>3$ \\
\hline protons & $>3$ & $>3$ & $<1.5$ \\
\hline
\end{tabular}

* 2 variation

\begin{tabular}{|c|c|c|c|}
\hline particle type & $\left|\mathrm{n} \sigma_{\mathrm{TPC}_{\pi}}\right|$ & $\left|\mathrm{n} \sigma_{\mathrm{TPC}_{\mathrm{K}}}\right|$ & $\left|\mathrm{n} \sigma_{\mathrm{TPC}_{\mathrm{p}}}\right|$ \\
\hline pions & $<3$ & $>3$ & $>3$ \\
\hline kaons & $>3$ & $<3$ & $>3$ \\
\hline protons & $>3$ & $>3$ & $<3$ \\
\hline
\end{tabular}

* 3 variation

\begin{tabular}{|c|c|c|c|}
\hline particle type & $\left|n \sigma_{\mathrm{TPC}_{\pi}}\right|$ & $\left|\mathrm{n} \sigma_{\mathrm{TPC}_{\mathrm{K}}}\right|$ & $\left|\mathrm{n} \sigma_{\mathrm{TPC}_{\mathrm{p}}}\right|$ \\
\hline pions & $<2$ & - & - \\
\hline kaons & - & $<2$ & - \\
\hline protons & - & - & $<2$ \\
\hline
\end{tabular}

$-p_{T}>0.5 \mathrm{GeV} / \mathrm{c}$ :

* 1 variation

\begin{tabular}{|c|c|c|c|}
\hline particle type & $\left|\mathrm{n} \sigma_{\mathrm{comb}_{\pi}}\right|$ & $\left|\mathrm{n} \sigma_{\mathrm{comb}_{K}}\right|$ & $\left|\mathrm{n} \sigma_{\mathrm{comb}_{\mathrm{p}}}\right|$ \\
\hline pions & $<1.5$ & $>3$ & $>3$ \\
\hline kaons & $>3$ & $<1.5$ & $>3$ \\
\hline protons & $>3$ & $>3$ & $<1.5$ \\
\hline
\end{tabular}


* 2 variation

\begin{tabular}{|c|c|c|c|}
\hline particle type & $\left|\mathrm{n} \sigma_{\mathrm{Comb}_{\pi}}\right|$ & $\left|\mathrm{n} \sigma_{\mathrm{comb}_{K}}\right|$ & $\left|\mathrm{n} \sigma_{\mathrm{Comb}_{\mathrm{p}}}\right|$ \\
\hline pions & $<3$ & $>3$ & $>3$ \\
\hline kaons & $>3$ & $<3$ & $>3$ \\
\hline protons & $>3$ & $>3$ & $<3$ \\
\hline
\end{tabular}

* 3 variation

\begin{tabular}{|c|c|c|c|}
\hline particle type & $\left|\mathrm{n} \sigma_{\mathrm{Comb}_{\pi}}\right|$ & $\left|\mathrm{n} \sigma_{\mathrm{comb}_{\mathrm{K}}}\right|$ & $\left|\mathrm{n} \sigma_{\mathrm{Comb}_{\mathrm{p}}}\right|$ \\
\hline pions & $<2$ & - & - \\
\hline kaons & - & $<2$ & - \\
\hline protons & - & - & $<2$ \\
\hline
\end{tabular}

The calculated purities and PID efficiencies obtained for pions, kaons and protons for the aforementioned PID strategy variations are shown in Figures 5.20, 5.21 and 5.22 , respectively. It was concluded that up to $2 \mathrm{GeV} / \mathrm{c}$ for pions and protons the looser n $\sigma$ acceptance criterium of the second strategy increased the efficiency with respect to the first one while purity stayed unaffected. For kaons the efficiency and contamination were both unaffected when changing the first strategy to the second one. In the third variation only the acceptance requirement was applied without the rejection part which was used in the first and the second strategies. This lead to a higher efficiency of around $80 \%$ and lower purity for all particles, but only above $2 \mathrm{GeV} / \mathrm{c}$, while for the studied range of $0.2-2 \mathrm{GeV} / \mathrm{c}$ purity remained the same.
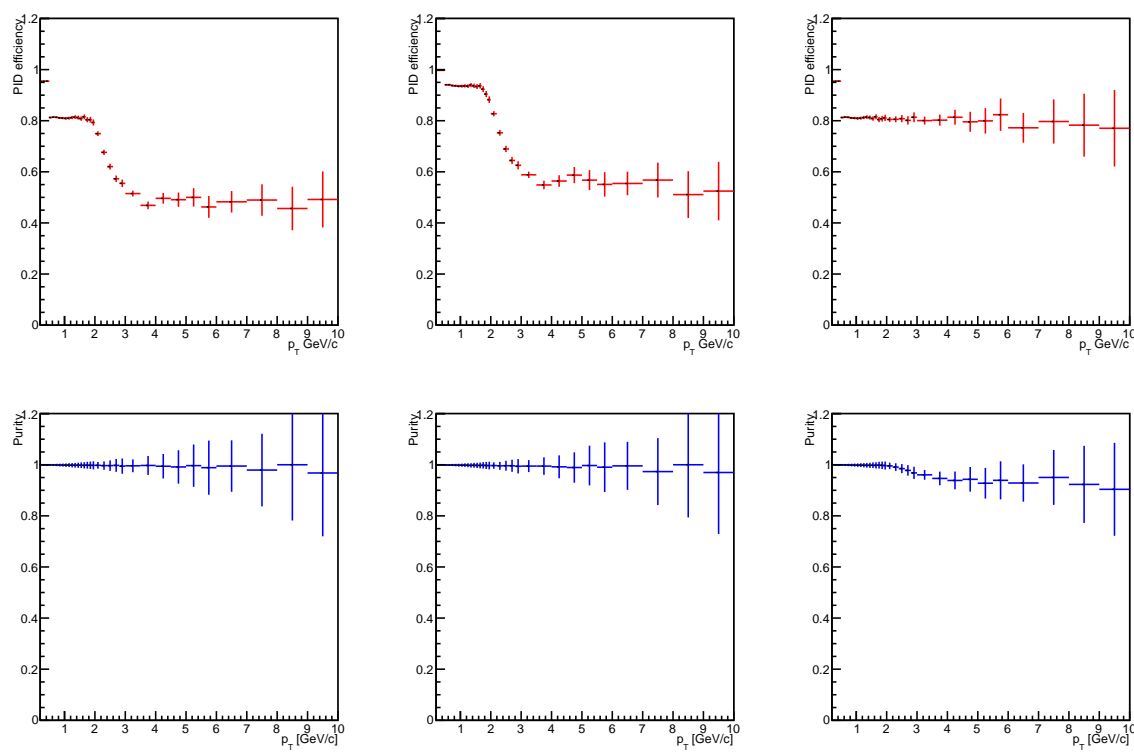

Figure 5.20: PID efficiencies and purities for pions reconstructed in the first (left), second (middle) and third (right) PID strategy variation 

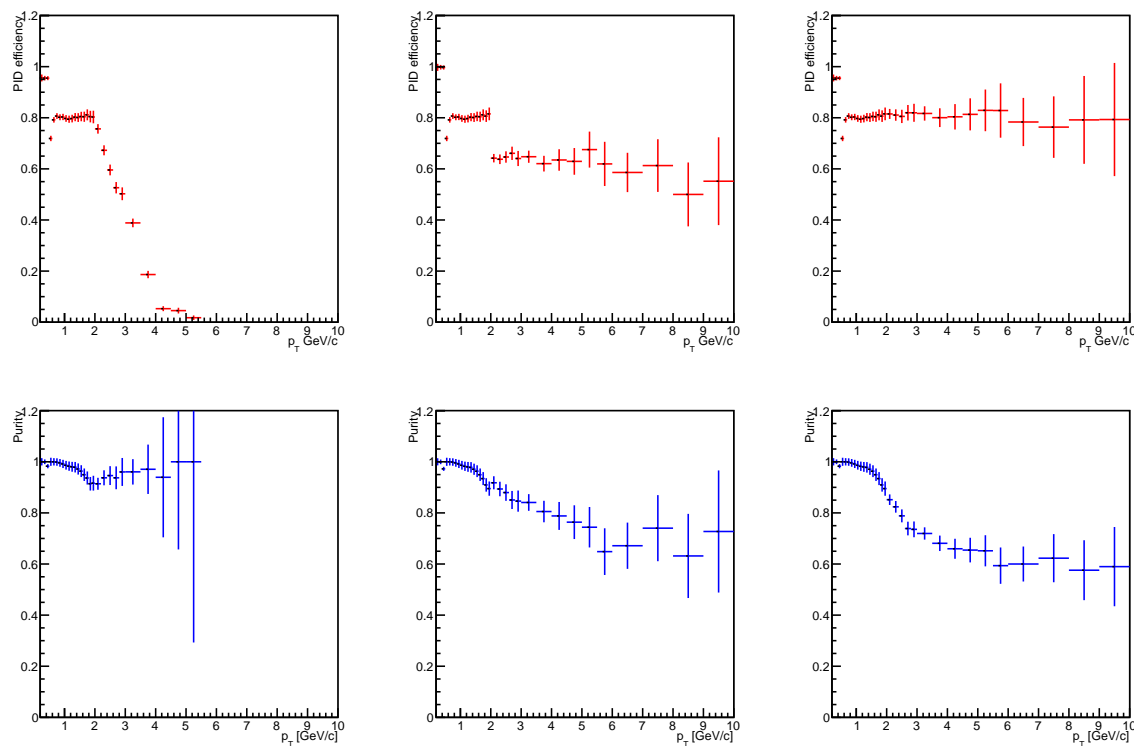

FiguRE 5.21: PID efficiencies and purities for kaons reconstructed in the first (left), second (middle) and third (right) PID strategy variation
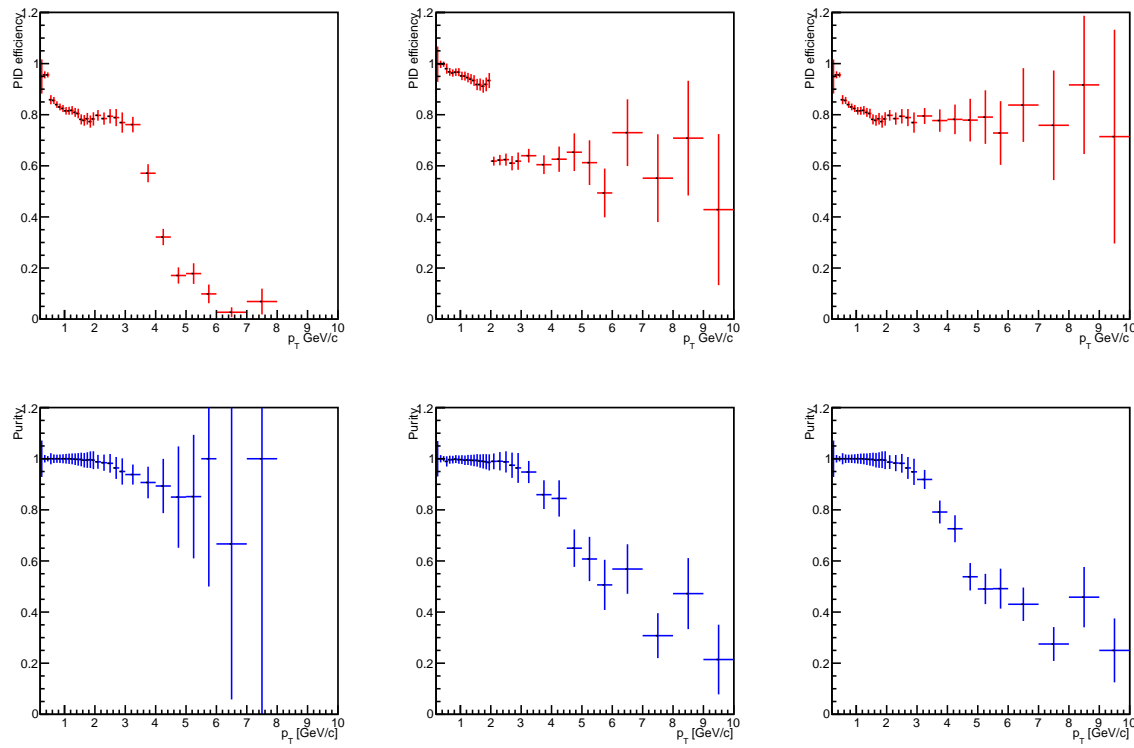

FiguRE 5.22: PID efficiencies and purities for protons reconstructed in the first (left), second (middle) and third (right) PID strategy variation

\subsubsection{Final values}

As explained in Section 5.1.2.1, the systematic uncertainty for every source was calculated as a half of the fit value to the maximum difference between the default and the trial measurement. The maximum contribution of every source to the total systematic uncertainty for every multiplicity bin for $\sigma_{\Delta y}$ and $\sigma_{\Delta \varphi}$ 
is summarised in Tables 5.1 and 5.2 for pions or Tables 5.3 and 5.4 for protons, respectively. In a same way, for the balance function projections it is presented in Table 5.5 for $\Delta \mathrm{y}$ and Table 5.6 for $\Delta \varphi$ for pions, while in Table 5.7 for $\Delta y$ and Table 5.8 for $\Delta \varphi$ for protons.

TABLE 5.1: Systematic uncertainties (in \%) for $\sigma_{\Delta y}$ for pions

\begin{tabular}{|c|c|c|c|c|c|c|c|c|}
\hline Multiplicity / Source & Sample & Bit & $\Delta \varphi^{*}$ & $\mathrm{~V}_{\mathrm{z}}$ & Pile-up & TPC clusters & $\chi^{2}$ & MC closure \\
\hline $0-5 \%$ & 0 & 0.6 & 0.025 & 0 & 0 & 0.1 & 0.02 & 0.2 \\
\hline $5-10 \%$ & 0 & 0.6 & 0.025 & 0 & 0 & 0.1 & 0.02 & 0.2 \\
\hline $10-20 \%$ & 0 & 0.6 & 0.025 & 0 & 0 & 0.1 & 0.02 & 0.2 \\
\hline $20-30 \%$ & 0 & 0.6 & 0.025 & 0 & 0 & 0.1 & 0.02 & 0.2 \\
\hline $30-40 \%$ & 0 & 0.6 & 0.025 & 0 & 0 & 0.1 & 0.02 & 0.2 \\
\hline $40-50 \%$ & 0 & 0.6 & 0.025 & 0 & 0 & 0.1 & 0.02 & 0.2 \\
\hline $50-60 \%$ & 0 & 0.6 & 0.025 & 0 & 0 & 0.1 & 0.02 & 0.2 \\
\hline $60-70 \%$ & 0 & 0.6 & 0.025 & 0 & 0 & 0.1 & 0.02 & 0.2 \\
\hline $70-80 \%$ & 0 & 0.6 & 0.025 & 0 & 0 & 0.1 & 0.02 & 0.2 \\
\hline
\end{tabular}

TABLE 5.2: Systematic uncertainties (in $\%$ ) for $\sigma_{\Delta \varphi}$ for pions

\begin{tabular}{|c|c|c|c|c|c|c|c|c|}
\hline Multiplicity / Source & Sample & Bit & $\Delta \varphi^{*}$ & $\mathrm{~V}_{\mathrm{z}}$ & Pile-up & TPC clusters & $\chi^{2}$ & MC closure \\
\hline $0-5 \%$ & 0 & 1 & 0.03 & 0 & 0 & 0.15 & 0.04 & 0.2 \\
\hline $5-10 \%$ & 0 & 1 & 0.03 & 0 & 0 & 0.15 & 0.04 & 0.2 \\
\hline $10-20 \%$ & 0 & 1 & 0.03 & 0 & 0 & 0.15 & 0.04 & 0.2 \\
\hline $20-30 \%$ & 0 & 1 & 0.03 & 0 & 0 & 0.15 & 0.04 & 0.2 \\
\hline $30-40 \%$ & 0 & 1 & 0.03 & 0 & 0 & 0.15 & 0.04 & 0.2 \\
\hline $40-50 \%$ & 0 & 1 & 0.03 & 0 & 0 & 0.15 & 0.04 & 0.2 \\
\hline $50-60 \%$ & 0 & 1 & 0.03 & 0 & 0 & 0.15 & 0.04 & 0.2 \\
\hline $60-70 \%$ & 0 & 1 & 0.03 & 0 & 0 & 0.15 & 0.04 & 0.2 \\
\hline $70-80 \%$ & 0 & 1 & 0.03 & 0 & 0 & 0.15 & 0.04 & 0.2 \\
\hline
\end{tabular}

TABle 5.3: Systematic uncertainties (in $\%$ ) for $\sigma_{\Delta y}$ for protons

\begin{tabular}{|c|c|c|c|c|c|c|c|c|}
\hline Multiplicity / Source & Sample & Bit & $\Delta \varphi^{*}$ & $\mathrm{~V}_{\mathrm{z}}$ & Pile-up & TPC clusters & $\chi^{2}$ & MC closure \\
\hline $0-5 \%$ & 0 & 0.35 & 0.05 & 0 & 0 & 0 & 0 & 0.2 \\
\hline $5-10 \%$ & 0 & 0.35 & 0.05 & 0 & 0 & 0 & 0 & 0.2 \\
\hline $10-20 \%$ & 0 & 0.35 & 0.05 & 0 & 0 & 0 & 0 & 0.2 \\
\hline $20-30 \%$ & 0 & 0.35 & 0.05 & 0 & 0 & 0 & 0 & 0.2 \\
\hline $30-40 \%$ & 0 & 0.35 & 0.05 & 0 & 0 & 0 & 0 & 0.2 \\
\hline $40-50 \%$ & 0 & 0.35 & 0.05 & 0 & 0 & 0 & 0 & 0.2 \\
\hline $50-60 \%$ & 0 & 0.35 & 0.05 & 0 & 0 & 0 & 0 & 0.2 \\
\hline $60-70 \%$ & 0 & 0.35 & 0.05 & 0 & 0 & 0 & 0 & 0.2 \\
\hline $70-80 \%$ & 0 & 0.35 & 0.05 & 0 & 0 & 0 & 0 & 0.2 \\
\hline
\end{tabular}


TABLE 5.4: Systematic uncertainties (in $\%$ ) for $\sigma_{\Delta \varphi}$ for protons

\begin{tabular}{|c|c|c|c|c|c|c|c|c|}
\hline Multiplicity / Source & Sample & Bit & $\Delta \varphi^{*}$ & $\mathrm{~V}_{\mathrm{z}}$ & Pile-up & TPC clusters & $\chi^{2}$ & MC closure \\
\hline $0-5 \%$ & 0 & 0.02 & 0.025 & 0 & 0 & 0.07 & 0.018 & 0.2 \\
\hline $5-10 \%$ & 0 & 0.02 & 0.025 & 0 & 0 & 0.07 & 0.018 & 0.2 \\
\hline $10-20 \%$ & 0 & 0.02 & 0.025 & 0 & 0 & 0.07 & 0.018 & 0.2 \\
\hline $20-30 \%$ & 0 & 0.02 & 0.025 & 0 & 0 & 0.07 & 0.018 & 0.2 \\
\hline $30-40 \%$ & 0 & 0.02 & 0.025 & 0 & 0 & 0.07 & 0.018 & 0.2 \\
\hline $40-50 \%$ & 0 & 0.02 & 0.025 & 0 & 0 & 0.07 & 0.018 & 0.2 \\
\hline $50-60 \%$ & 0 & 0.02 & 0.025 & 0 & 0 & 0.07 & 0.018 & 0.2 \\
\hline $60-70 \%$ & 0 & 0.02 & 0.025 & 0 & 0 & 0.07 & 0.018 & 0.2 \\
\hline $70-80 \%$ & 0 & 0.02 & 0.025 & 0 & 0 & 0.07 & 0.018 & 0.2 \\
\hline
\end{tabular}

TABLE 5.5: Systematic uncertainties (in \%) for balance function projection in $\Delta y$ for pions

\begin{tabular}{|c|c|c|c|c|c|c|c|c|}
\hline Multiplicity / Source & Sample & Bit & $\Delta \varphi^{*}$ & $\mathrm{~V}_{\mathrm{z}}$ & Pile-up & TPC clusters & $\chi^{2}$ & MC closure \\
\hline $0-5 \%$ & 0.02 & 10 & 2 & 0.25 & 0.1 & 2 & 0.02 & 0.05 \\
\hline $30-40 \%$ & 0.49 & 8 & 2 & 0.3 & 0.008 & 1.5 & 0.02 & 0.05 \\
\hline $70-80 \%$ & 0.12 & 10 & 1.5 & 0.35 & 0.05 & 1.5 & 0.007 & 0.05 \\
\hline
\end{tabular}

TABLE 5.6: Systematic uncertainties (in \%) for balance function projection in $\Delta \varphi$ for pions

\begin{tabular}{|c|c|c|c|c|c|c|c|c|}
\hline Multiplicity / Source & Sample & Bit & $\Delta \varphi^{*}$ & $\mathrm{~V}_{\mathrm{z}}$ & Pile-up & TPC clusters & $\chi^{2}$ & MC closure \\
\hline $0-5 \%$ & 0.18 & 15 & 0.1 & 0.1 & 0.2 & 2.5 & 0.035 & 0.39 \\
\hline $30-40 \%$ & 0.7 & 15 & 0.05 & 0.35 & 0.02 & 2 & 0.09 & 0.39 \\
\hline $70-80 \%$ & 0.04 & 20 & 0.03 & 0.45 & 0.09 & 3 & 0.12 & 0.39 \\
\hline
\end{tabular}

TABLE 5.7: Systematic uncertainties (in \%) for balance function projection in $\Delta \mathrm{y}$ for protons

\begin{tabular}{|c|c|c|c|c|c|c|c|c|}
\hline Multiplicity / Source & Sample & Bit & $\Delta \varphi^{*}$ & $\mathrm{~V}_{\mathrm{z}}$ & Pile-up & TPC clusters & $\chi^{2}$ & MC closure \\
\hline $0-5 \%$ & 0.1 & 5.5 & 4 & 0.1 & 0.15 & 0.6 & 0.2 & 0.2 \\
\hline $30-40 \%$ & 0.22 & 6.5 & 4 & 0.25 & 0.045 & 0.7 & 0.1 & 0.2 \\
\hline $70-80 \%$ & 0.94 & 5 & 6 & 0.45 & 0.22 & 0.7 & 0.2 & 0.2 \\
\hline
\end{tabular}


TABLE 5.8: Systematic uncertainties (in \%) for balance function projection in $\Delta \varphi$ for protons

\begin{tabular}{|c|c|c|c|c|c|c|c|c|}
\hline Multiplicity / Source & Sample & Bit & $\Delta \varphi^{*}$ & $\mathrm{~V}_{\mathrm{z}}$ & Pile-up & TPC clusters & $\chi^{2}$ & MC closure \\
\hline $0-5 \%$ & 3.6 & 3.8 & 0.5 & 0.05 & 0.9 & 1.2 & 0.6 & 1 \\
\hline $30-40 \%$ & 0.4 & 5.3 & 0.05 & 1 & 0.45 & 0.47 & 0.04 & 1 \\
\hline $70-80 \%$ & 1.45 & 4 & 0.4 & 2.4 & 0.9 & 0.25 & 0.19 & 1 \\
\hline
\end{tabular}

\subsubsection{Balance function projections}

Figure 5.23 shows the projections of the two-dimensional distributions for charged particles for the same multiplicity classes on $\Delta \eta$, while Figure $5.24-$ on $\Delta \varphi$, obtained as explained in Section 4.3 with only statistical uncertainties indicated by the error bars.
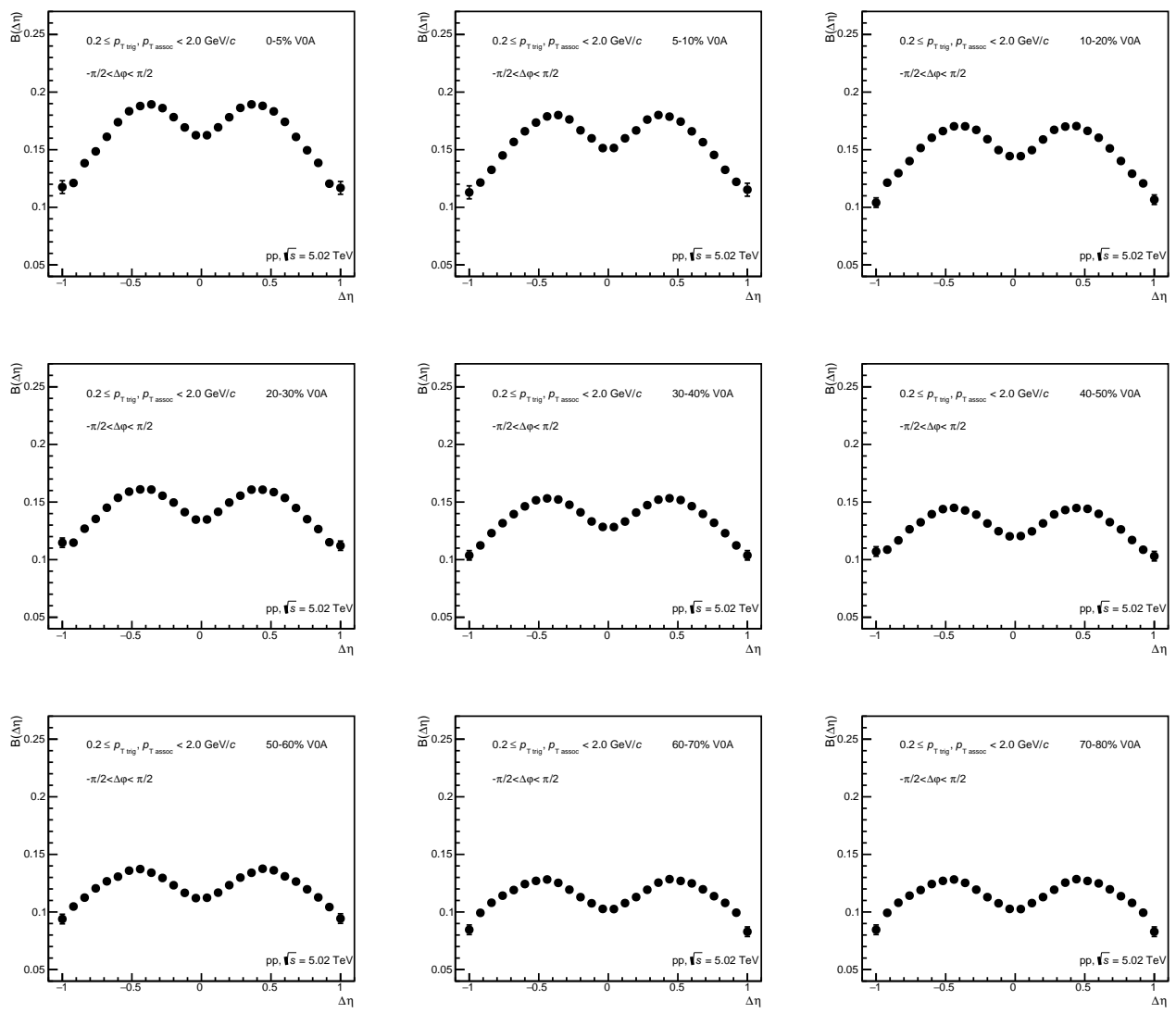

FigURE 5.23: Balance function projections on $\Delta \eta$ of charged particles in pp collisions at $\sqrt{\mathrm{s}}=5.02 \mathrm{TeV}$ as a function of multiplicity class 

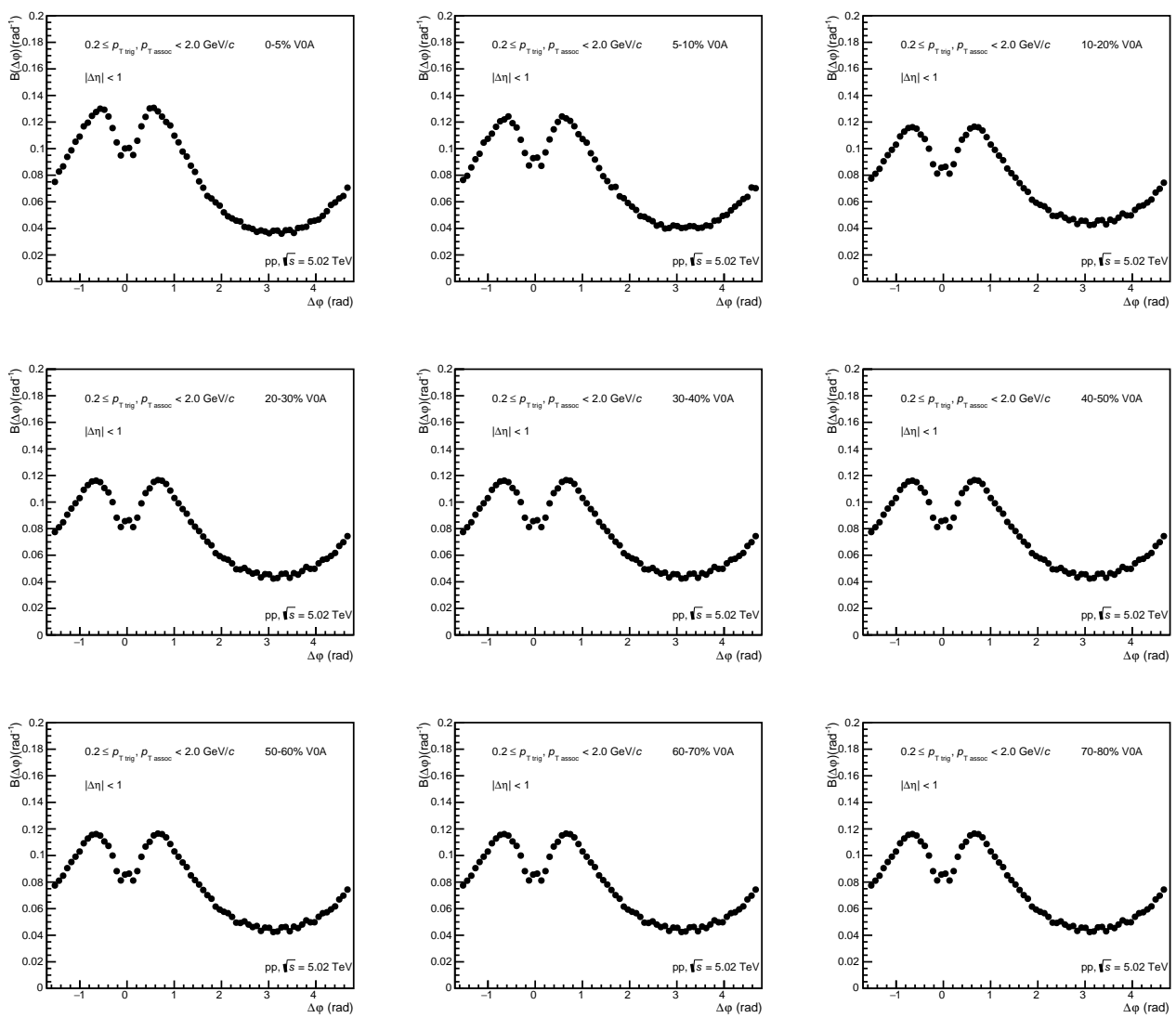

Figure 5.24: Balance function projections on $\Delta \varphi$ of charged particles in pp collisions at $\sqrt{\mathrm{s}}=5.02 \mathrm{TeV}$ as a function of multiplicity class

To demonstrate the differences better, projections for three selected multiplicity classes of $0-5 \%, 30-40 \%$ and $70-80 \%$ are shown together in Figure 5.25 including the systematic uncertainties indicated by the open boxes.

It can be seen that the magnitude of the balance function is decreasing towards low multiplicity events signalling a smaller amount of correlated pairs, while the away side becomes slightly more pronounced. The near side of the balance function distribution becomes narrower for higher multiplicity that is reflected better in the results of the balance function width following later in the text.

The systematic uncertainty values were considered to be equal to the uncertainties of the balance function projections measured for pions at the same energy and shown in Figure 5.44 since no big difference is expected due to the fact that pions form the majority of the charged particle sample. The procedure and sources considered for the systematic uncertainty estimation for balance function projections are described in detail in Section 5.1.2. 

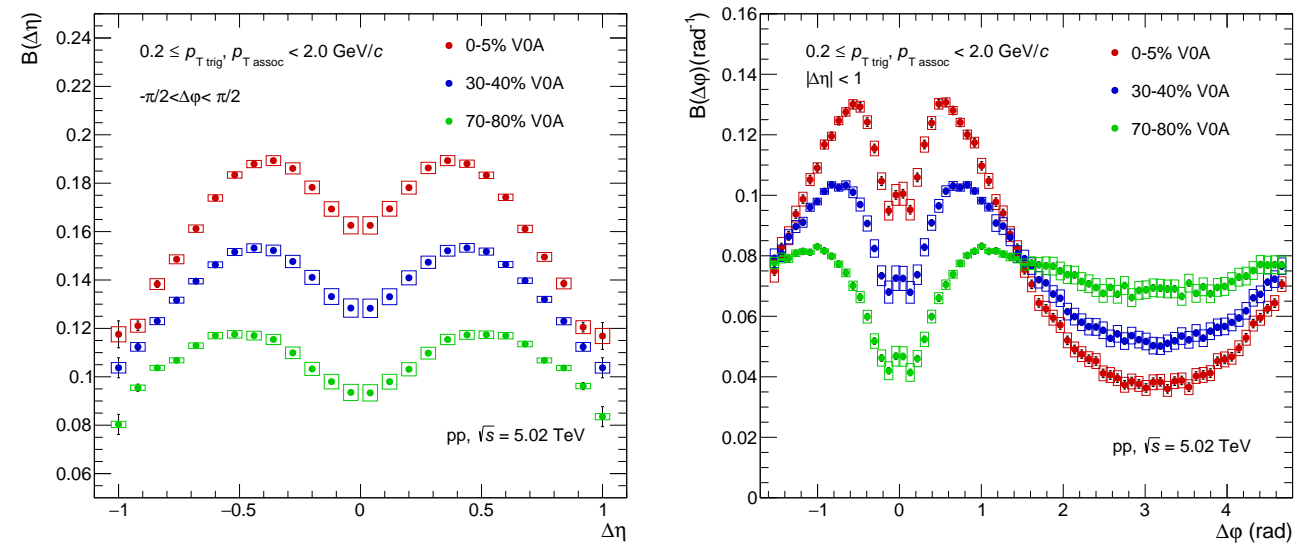

FigURE 5.25: Balance function projections on $\Delta \eta$ (left) and $\Delta \varphi$ (right) of charged particles in pp collisions at $\sqrt{\mathrm{s}}=5.02 \mathrm{TeV}$ for $0-5 \%, 30-40 \%$ and $70-80 \%$ multiplicity classes

\subsubsection{Balance function width and yield}

The balance function widths $\sigma_{\Delta \eta}$ and $\sigma_{\Delta \varphi}$ as well as the total, near- and awayside yields $\mathrm{Y}_{\mathrm{B}_{\text {(total) }},} \mathrm{Y}_{\mathrm{B}_{\text {(near) }}}$ and $\mathrm{Y}_{\mathrm{B}_{(\text {away }}}$ are obtained from the aforementioned projections according to the procedure described in Section 4.3.

The results of the yield and the width are shown in Figures 5.26 and 5.27 together with the statistical and systematic uncertainties indicated by the error bars and the open boxes, respectively. In a similar way as it was done for the projections, the systematic uncertainties were considered to be equal to the uncertainties of the pion balance function measured at the same energy. The values of the yield mentioned in the text below are given in terms of relative values, defined for the total calculated yield as $\mathrm{Y}_{\mathrm{r}_{\text {total }}}=\frac{\mathrm{Y}_{\text {total }}}{\mathrm{Y}_{\max }} \cdot 100 \%$, where $\mathrm{Y}_{\max }=1$ is the maximum possible total yield. For the away- and the near-side yields the relative values are calculated with respect to the total calculated yield as $\mathrm{Y}_{\mathrm{r}_{\text {away (near) }}}=\frac{\mathrm{Y}_{\text {away (near) }}}{\mathrm{Y}_{\text {total }}} \cdot 100 \%$.

The yield of the balance function allows to access the number of the correlated particle pairs with an opposite charge in a certain part in the acceptance of the measurement. As can be seen in Figure 5.26, the total yield of the balance function for charged particles remains at the level of 48-50\% depending on the multiplicity class. This value means that around $48-50 \%$ of the correlated pairs are reconstructed in a given acceptance range of $|y|<0.5$. From the values of the away- and the near-side yields it is clear that around $70 \%$ of the total measured correlation is located at the near side and only $30 \%$ at the away side for the highest multiplicity class. For the lowest multiplicity class this is distributed almost equally at around 50\%. Moreover, the total yield becomes slightly higher 
for the higher multiplicity class. This can be explained by the fact that the balance function distribution becomes broader at lower multiplicities. This leads to a part of the correlated pairs falling outside the detector acceptance range and not being taken into account. However, this might also be an effect of larger amount of balancing charges being produced in the higher multiplicity with respect to the lower multiplicity class.
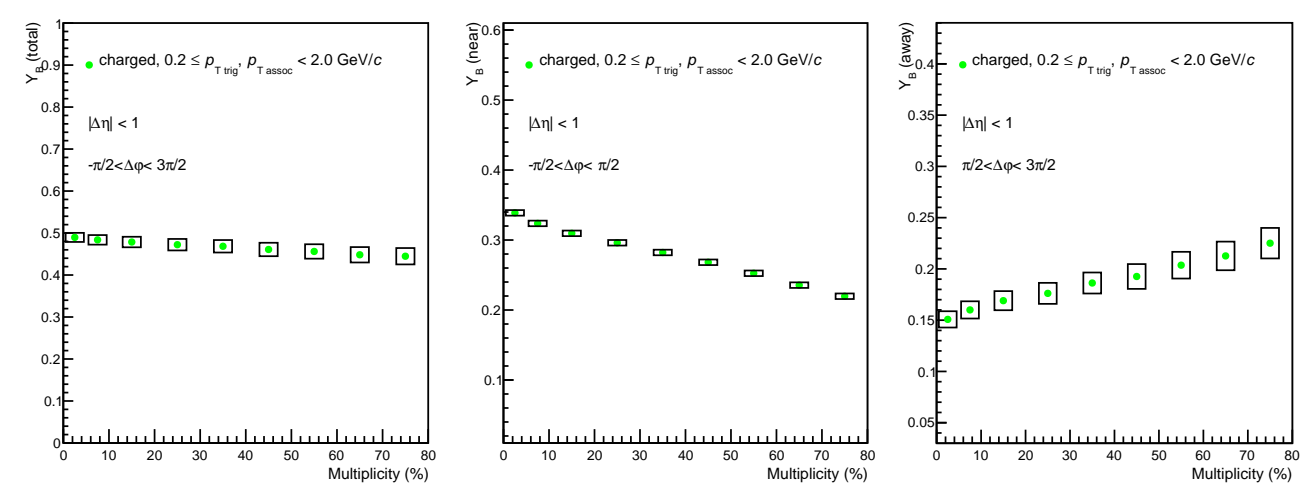

FIGURE 5.26: Total (left), near-side (middle) and away-side (right) yields of charged particle balance function in pp collisions at $\sqrt{\mathrm{s}}=5.02 \mathrm{TeV}$ as a function of multiplicity class

In Figure 5.27, a significant multiplicity-dependent narrowing of the width can be observed in both dimensions. To make sure that this behaviour is in agreement with previous results observed in pp collisions, two different comparisons to the published results at $\sqrt{\mathrm{s}}=7 \mathrm{TeV}[111]$ were performed.

In the first one, the same analysis was repeated at the energy of $\sqrt{\mathrm{s}}=7 \mathrm{TeV}$ using the previous data collected in LHC Run 1, but the recent version of the code. The balance function width obtained in both cases is shown in Figure 5.28 with no systematic uncertainties for the repeated analysis, while the ratio between the result of repeated analysis over the published one is presented in Figure 5.29. From the fit values $\left(1.0039+/-0.0007\right.$ for $\sigma_{\Delta \eta}$ and $1.0026+/-0.0008$ for $\left.\sigma_{\Delta \varphi}\right)$ it can be seen that the difference is very small being of the order of $0.3 \%$ well within the systematic uncertainties of the measurement.

The second comparison was done by repeating the analysis presented in this thesis at $\sqrt{\mathrm{s}}=5.02 \mathrm{TeV}$ but using the same event and track selection including pseudorapidity range of $|\eta|<0.8$ as was used for the published measurement instead of $|\eta|<0.5$. Figure 5.30 shows the results with the systematic uncertainties included for both cases, while the corresponding ratio between the results of this analysis over the published data is presented in Figure 5.31. As can be seen from the values of the constant function fit to the ratio $\left(1.0304+/-0.0007\right.$ for $\sigma_{\Delta \eta}$ 
and $1.0594+/-0.0009$ for $\sigma_{\Delta \varphi}$ ), the difference around $3 \%$ and $6 \%$ is observed for $\sigma_{\Delta \eta}$ and $\sigma_{\Delta \varphi}$ respectively. This difference is presumably caused by the difference in the energy. In heavy-ion collisions higher energy leads to higher radial flow (see Chapter 1 for more details). Similarly, effects in pp collisions that resemble collective motion could depend on energy. This results in a stronger angular correlation, or a narrower balance function width in each multiplicity class at 7 $\mathrm{TeV}$ with respect to $5.02 \mathrm{TeV}$ results.
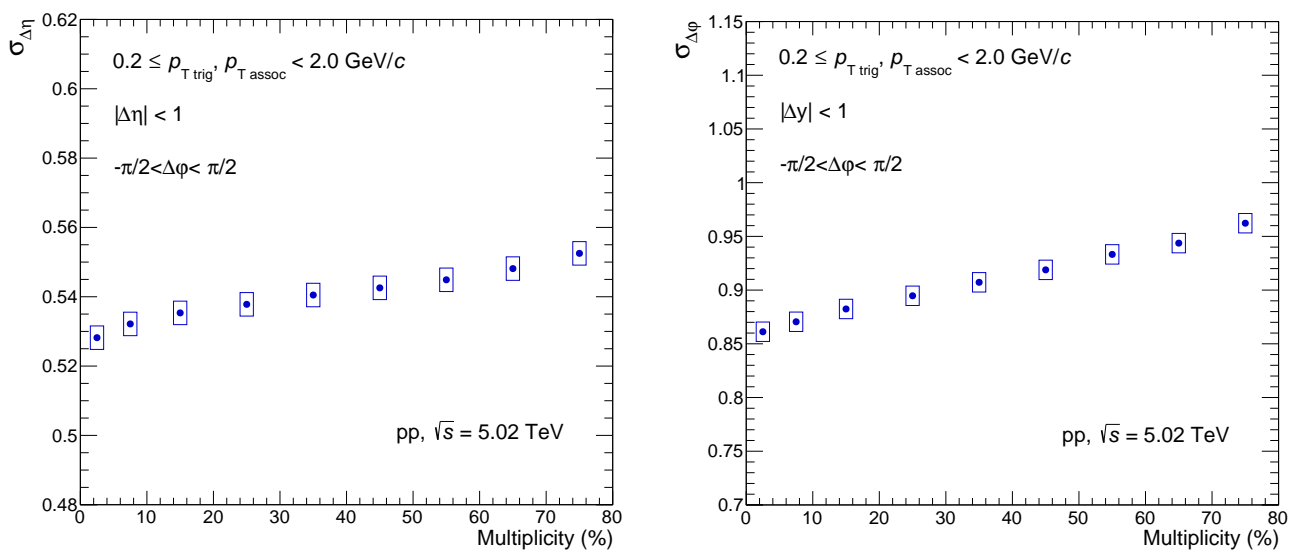

FiguRE 5.27: Balance function width $\sigma_{\Delta \eta}$ (left) and $\sigma_{\Delta \varphi}$ (right) of charged particles in pp collisions at $\sqrt{\mathrm{s}}=5.02 \mathrm{TeV}$ as a function of multiplicity class
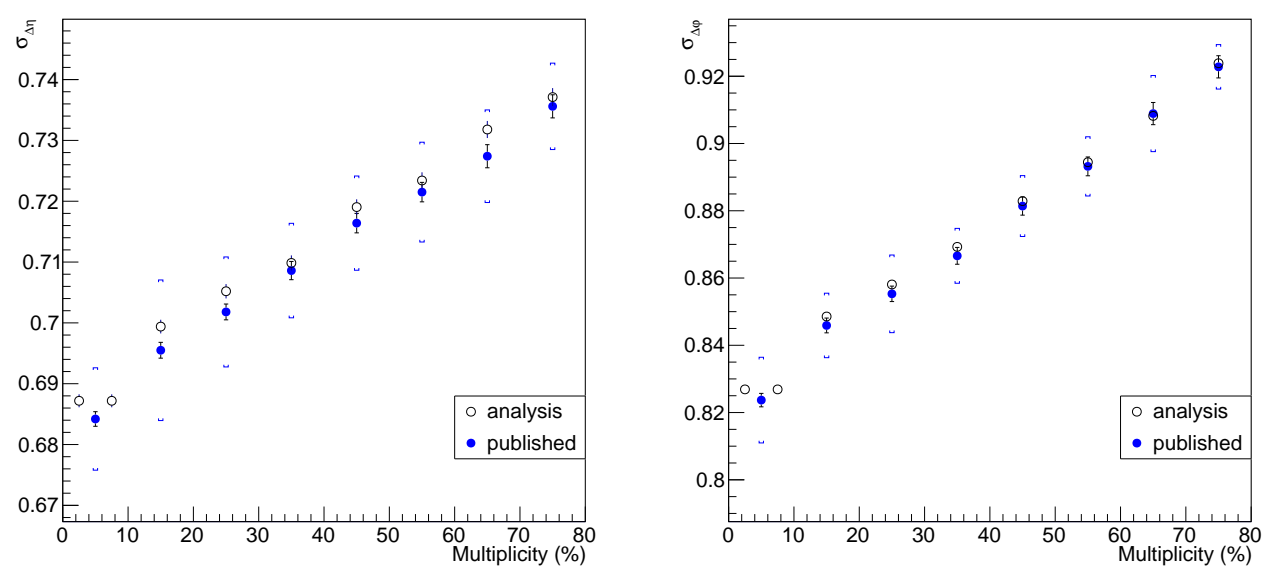

FIgURE 5.28: Comparison between the balance function width in $\Delta \eta$ (left) and $\Delta \varphi$ (right) in repeated analysis at $\sqrt{\mathrm{s}}=7 \mathrm{TeV}$ and published results for charged particles at $\sqrt{\mathrm{s}}=7 \mathrm{TeV}$ 

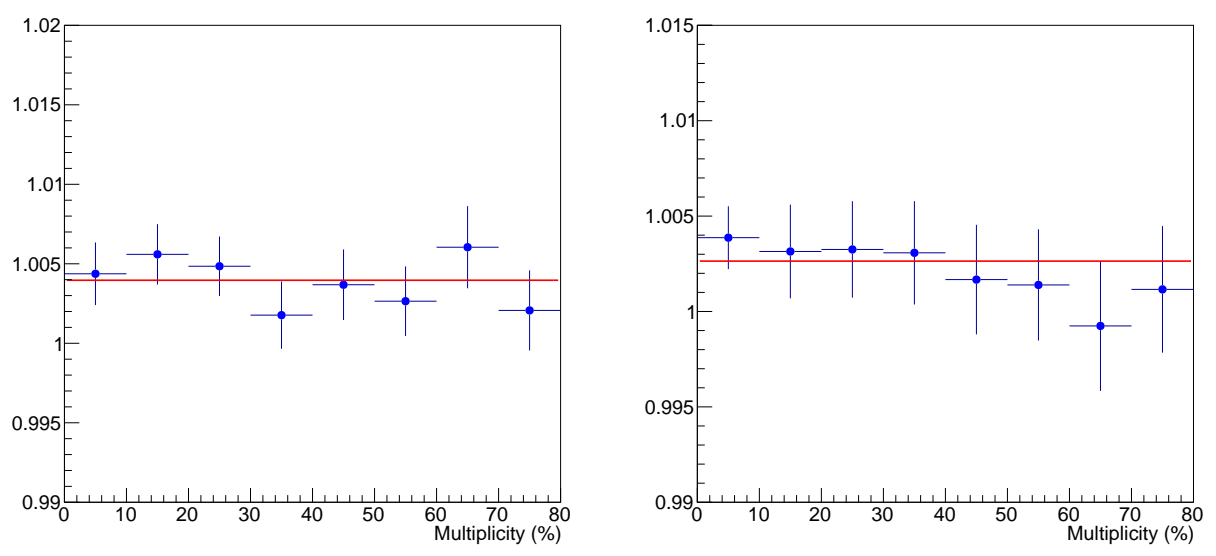

FIgURE 5.29: Ratio of the balance function width in $\Delta \eta$ (left) and $\Delta \varphi$ (right) in repeated analysis at $\sqrt{\mathrm{s}}=7 \mathrm{TeV}$ over published results for charged particles at $\sqrt{\mathrm{s}}=7 \mathrm{TeV}$
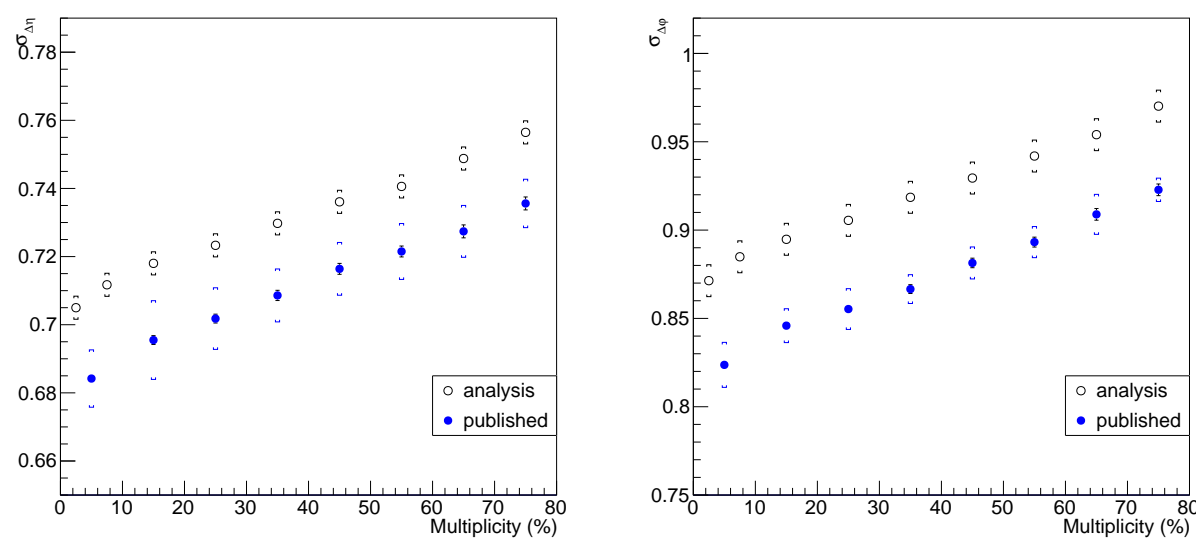

Figure 5.30: Comparison between the balance function width in $\Delta \eta$ (left) and $\Delta \varphi$ (right) obtained at $\sqrt{\mathrm{s}}=5.02 \mathrm{TeV}$ and published results for charged particles at $\sqrt{\mathrm{s}}=7 \mathrm{TeV}$ 

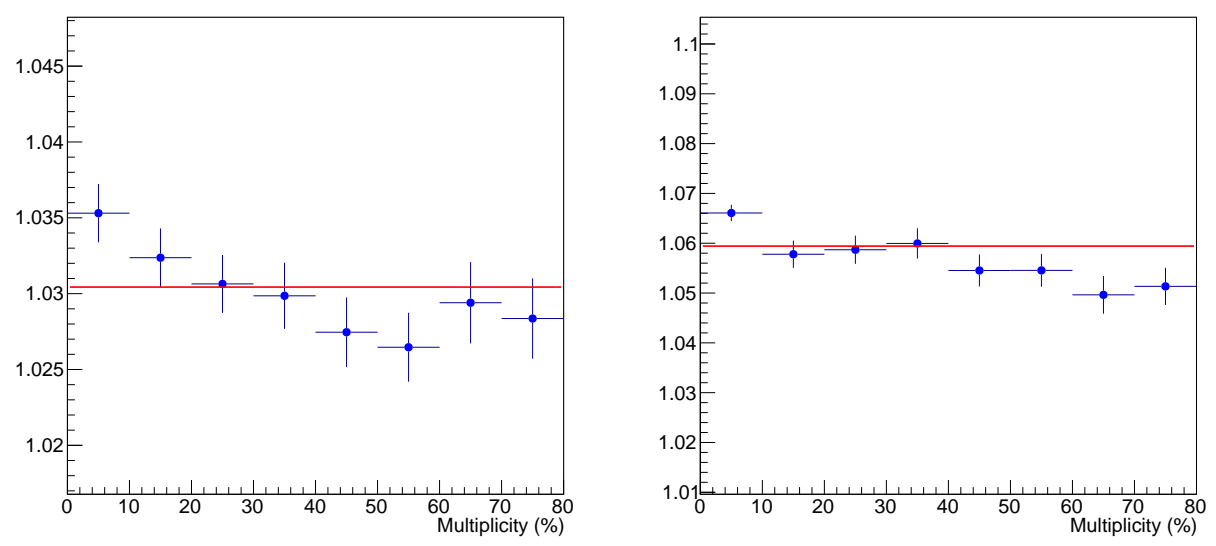

Figure 5.31: Ratio of the balance function width in $\Delta \eta$ (left) and $\Delta \varphi$ (right) obtained at $\sqrt{\mathrm{s}}=5.02 \mathrm{TeV}$ over published results for charged particles at $\sqrt{\mathrm{s}}=7 \mathrm{TeV}$

A qualitatively similar multiplicity-dependent narrowing of the width was measured in $\mathrm{Pb}-\mathrm{Pb}$ collisions at $\sqrt{\mathrm{S}_{\mathrm{NN}}}=2.76 \mathrm{TeV}$, where it was attributed to the higher radial flow in central with respect to peripheral events (see Chapter 1 for more details). This consequently leads to stronger final state correlations and hence narrower width for higher multiplicity [111]. It is important to point out that the depletion around $(\Delta \eta, \Delta \varphi)=(0,0)$ was excluded as a possible reason for such trend by observing that the trend didn't change after the removal of this structure, as explained in the beginning of this section. Since collective effects associated with the expanding quark-gluon plasma were not expected to be present in small systems, other mechanisms that resemble radial flow were proposed as an origin of this narrowing. One such mechanism for pp collisions is the color reconnection, introduced previously in Section 1.3 and described in detail in Section 5.2.5. Previous comparison of multiplicity-dependent results of the balance function width for charged particles in pp collisions at $\sqrt{\mathrm{s}}=7 \mathrm{TeV}$ with predictions from the PYTHIA8 model that included the color reconnection process showed a qualitative agreement with this assumption [111] (see Figure 2.13).

However, studying identified hadrons instead of charged particles allows more detailed investigation of the origin of such narrowing. This was the main goal of the presented analysis with the final results discussed in Section 5.2. 


\subsection{Balance function of pion, kaon and proton pairs in pp collisions at $\sqrt{\mathrm{s}}=5.02 \mathrm{TeV}$}

The main measurement in this analysis is done for identified particles to investigate more precisely the origin of the multiplicity-dependent narrowing of the balance function width in pp collisions. This can be addressed due to the dependence of the momentum of a certain particle on its mass that makes heavier particles more sensitive to the collective boost from radial flow or similar effects (see Equation 1.1). However, collective effects associated with the hydrodynamic expansion of the quark-gluon plasma are not expected to be present in the small systems. Alternative mechanisms were proposed in literature for small systems such as color reconnection that resemble a hydrodynamic boost and are referred as "radial flow-like" effects later on. These effects would lead to a similar dependence of the balance function measurement on the particle mass.

The analysis was performed for identified pions, kaons and protons. According to the previous assumption, it was expected to observe stronger correlation between protons with respect to, for instance, pions in every multiplicity class in pp collisions. In the measurement of the balance function width as a function of multiplicity, this would result in the observation of narrower widths for protons than for pions per multiplicity bin. Moreover, this would also mean more pronounced narrowing with increasing multiplicity for protons than for pions.

\subsubsection{Two-dimensional balance function distributions}

The results of the two-dimensional balance function distributions for 8 multiplicity classes from $0-5 \%$ to $70-80 \%$ for pions, kaons and protons measured in pp collisions at $\sqrt{\mathrm{s}}=5.02 \mathrm{TeV}$ are shown in Figures 5.32, 5.33 and 5.34 respectively. Different shapes and magnitudes can be observed depending on the particle type and multiplicity class.

In the balance function of pion pairs in Figures 5.32, similar depletion around $(\Delta \mathrm{y}, \Delta \varphi)=(0,0)$ is seen as in the previous measurement of charged particles described earlier in Section 5.1.1. The fact that it is not restricted to a very narrow window in either $\Delta \mathrm{y}$ or $\Delta \varphi$ indicates that the origin is not due to detector effects. Previous studies that used different cuts on $\Delta \mathrm{p}_{\mathrm{T}}$ of the particle pair allowed to conclude that the main sources contributing to this structure are presumably short-range correlations such as Bose-Einstein correlations of identical bosons or Coulomb attraction and repulsion [130]. Quantum statistics correlations of $\pi^{+} \pi^{+}$and $\pi^{-} \pi^{-}$pairs would lead to a higher like-sign per-trigger 
yield magnitudes around $(\Delta \mathrm{y}, \Delta \varphi)=(0,0)$ with respect to the unlike-sign part in the balance function definition (see Section 4.3) which will create a dip at the level of subtraction. Apart from these sources, in case of the analysis of identified pions an effect from resonances decaying into $\pi^{+} \pi^{-}$pairs can be more pronounced than in the charged particle analysis. The away side that becomes more pronounced for lower multiplicities might be as well caused by resonances decaying back-to-back. To study how exactly resonances can affect the shape of the balance function distribution of pions, Monte Carlo the simulation was used. The results of this simulation study are reported in Section 5.2.2.
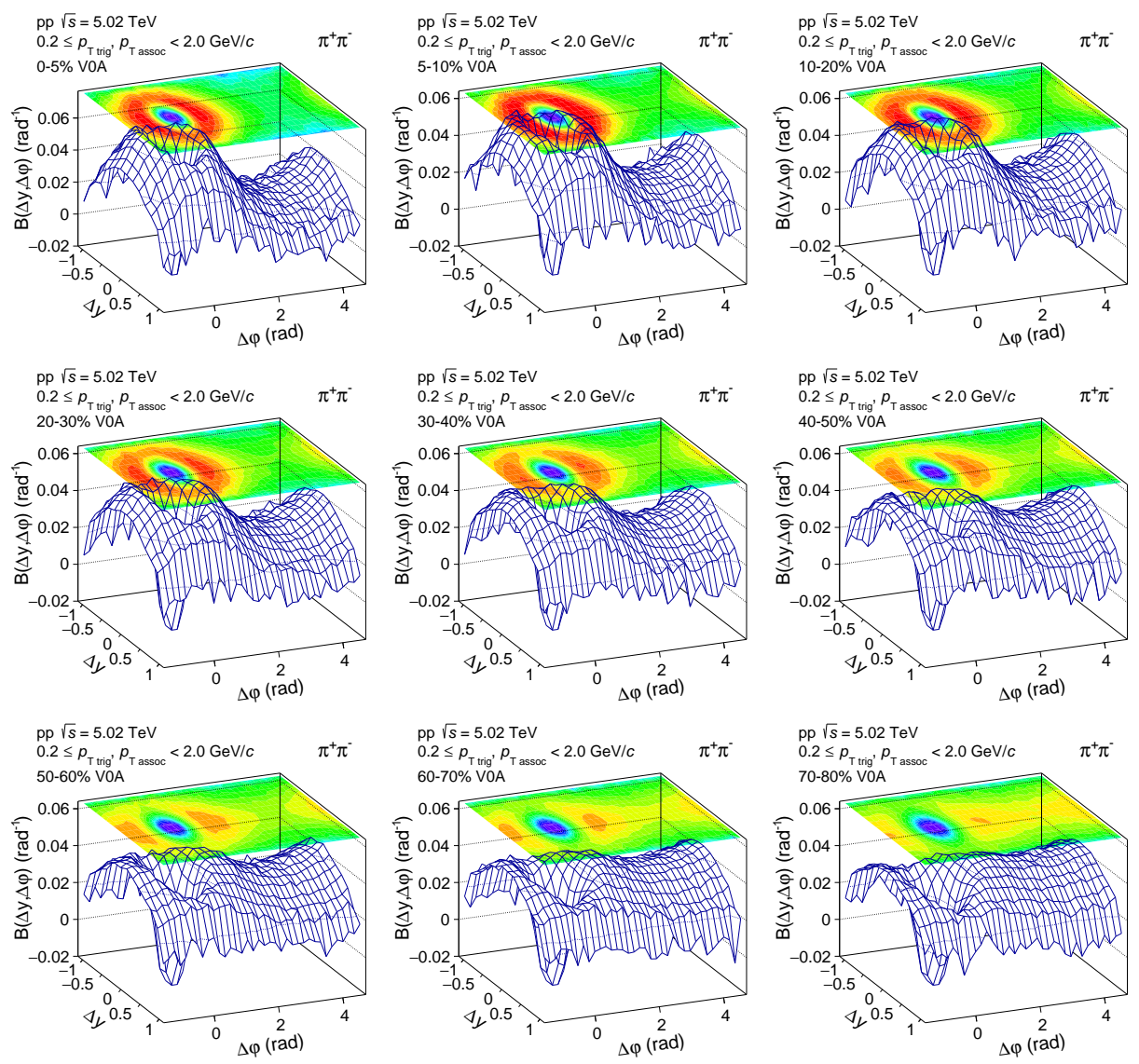

Figure 5.32: Two-dimensional balance function of pions in pp collisions at $\sqrt{\mathrm{s}}=5.02 \mathrm{TeV}$ as a function of multiplicity class

As seen in Figure 5.33, the balance function of kaon pairs is dominated by the near-side peak while the away side remains low for all multiplicity classes. In addition, it is much more narrow in comparison to pions in all multiplicity bins. The peak seems to be composed of two different shapes: the main peak and the shoulders extending to wider ranges of $\Delta y$. In the analysis at the generator level it was found that the large fraction of the near-side peak is caused by the $\phi$ resonance decaying into $\mathrm{K}^{+} \mathrm{K}^{-}$pairs (see Section 5.2.2). Since this contribution significantly affects the shape of kaon balance function, the final results for kaons 
should be reported with the $\phi$ meson being excluded in the data analysis. Therefore, the results of the balance function projections and the width are reported only for pions and protons.
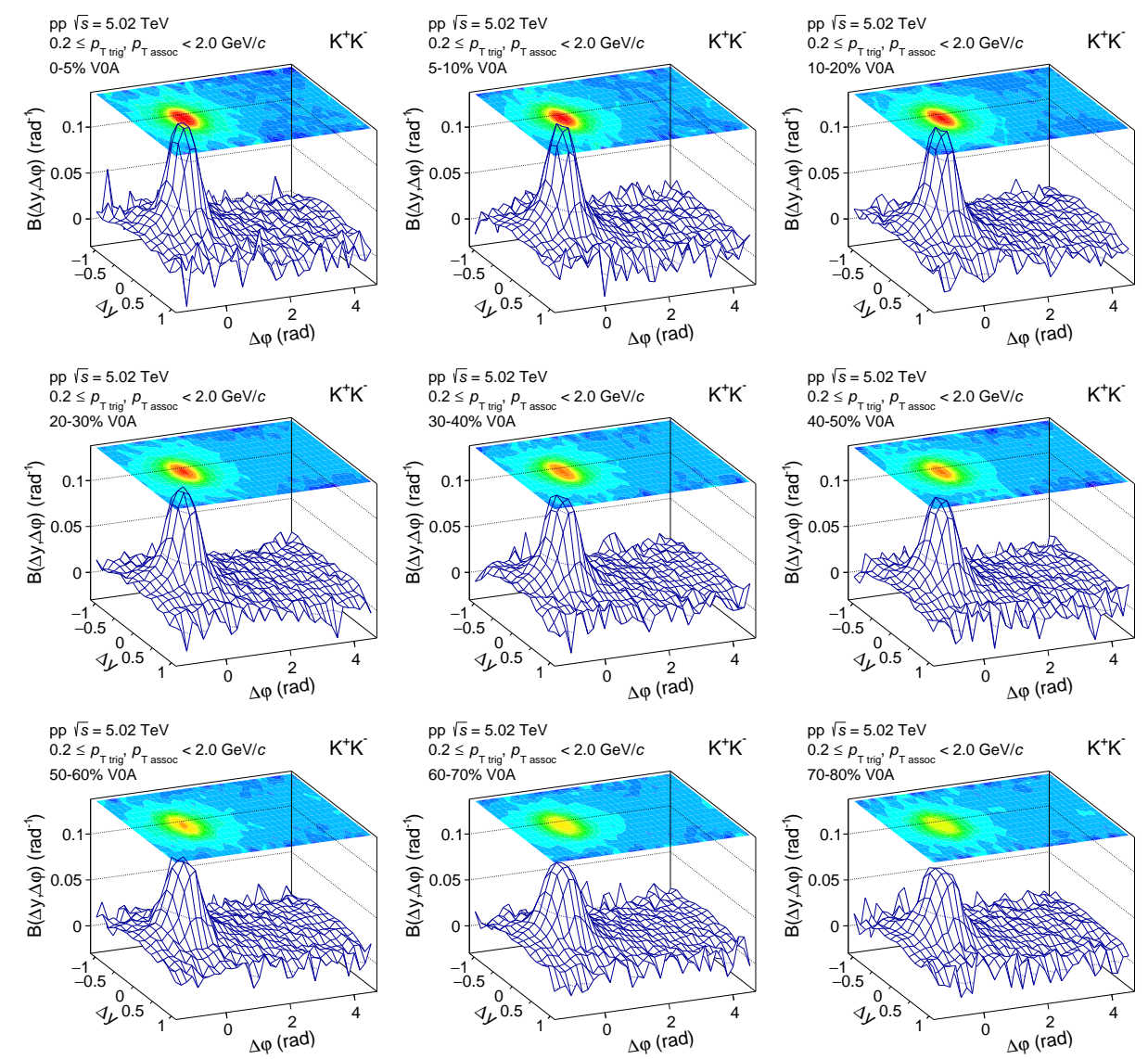

Figure 5.33: Two-dimensional balance function of kaons in pp collisions at $\sqrt{\mathrm{s}}=5.02 \mathrm{TeV}$ as a function of multiplicity class

The balance function of proton pairs, presented in Figure 5.34, has a pronounced near-side peak too. Since proton pairs will not have a contribution from BoseEinstein correlations, the like-sign per-trigger yield subtraction in the balance function definition would not lead to a depletion around $(\Delta \mathrm{y}, \Delta \varphi)=(0,0)$. The away side of the distributions does not show any significant value because no resonances decay into unlike-sign proton pairs. 

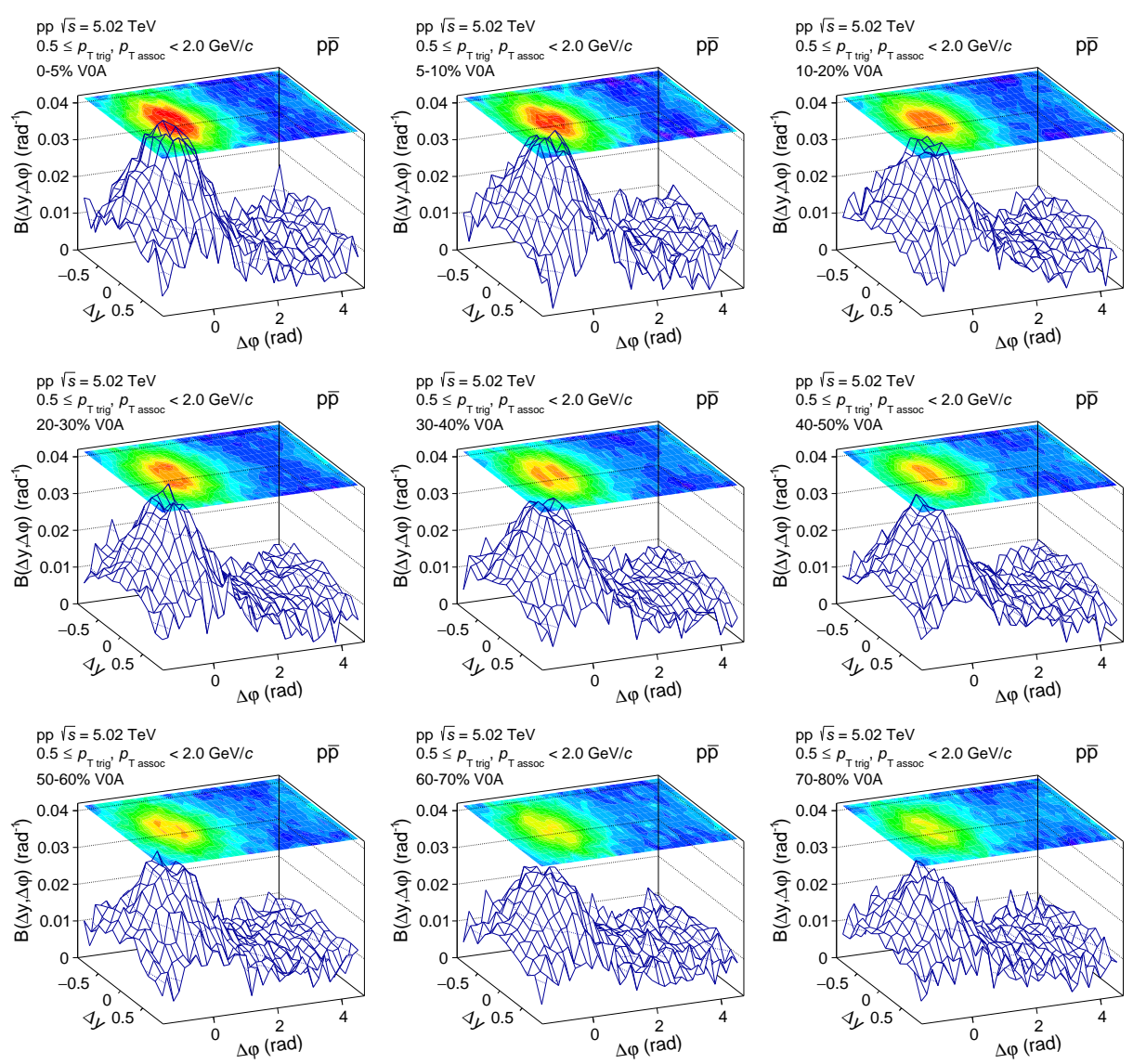

FiguRE 5.34: Two-dimensional balance function of protons in pp collisions at $\sqrt{\mathrm{s}}=5.02 \mathrm{TeV}$ as a function of multiplicity class

For all three particle species the magnitude of the balance function becomes smaller with decreasing multiplicity due to the smaller amount of the measured correlations.

\subsubsection{Studies of the resonance contribution in the balance func- tion of pions and kaons}

To estimate how resonances affect the shape of the presented balance function distribution of pion and kaon pairs, a simulation study at the Monte Carlo generator level was performed with the PYTHIA8 model. This was possible by using the known identity of every particle using the Monte Carlo particle data group (pdg) code that allows for the exclusion of an unwanted particle type from the analysis.

In particular, in case of pions the presented study helped to understand the origin of the near-side depletion at $(\Delta \mathrm{y}, \Delta \varphi)=(0,0)$ seen in Figure 5.32. It was found that the main contribution to this structure in the pion balance function is caused 
by resonance decays, namely $\omega$ and $\rho^{0}$ mesons. While the $\rho^{0}$ resonance decays into $\pi^{+}$and $\pi^{-}$with branching ratio of $\sim 100 \%$ [131], the $\omega$ resonance decay produces $\pi^{+}, \pi^{-}$and $\pi^{0} 89 \%$ of the times [131]. Balance function distributions of both resonances are presented in Figure 5.35. A similar depletion is observed in each distribution, however it is more pronounced in the $\rho$ balance function. In both cases this structure is caused by the decay kinematics. For $\rho$ mesons with low-momentum the produced $\pi^{+}$and $\pi^{-}$will travel more likely in opposite directions than very close to each other, therefore most of the correlation is seen on the away side. However, a peak on the near side just before the depletion is still present due to decays of $\rho$ mesons with higher momentum. As for the $\omega$ resonance, the $\pi^{-}$and $\pi^{+}$will be separated by some angle which will lead to the depletion on the near side.
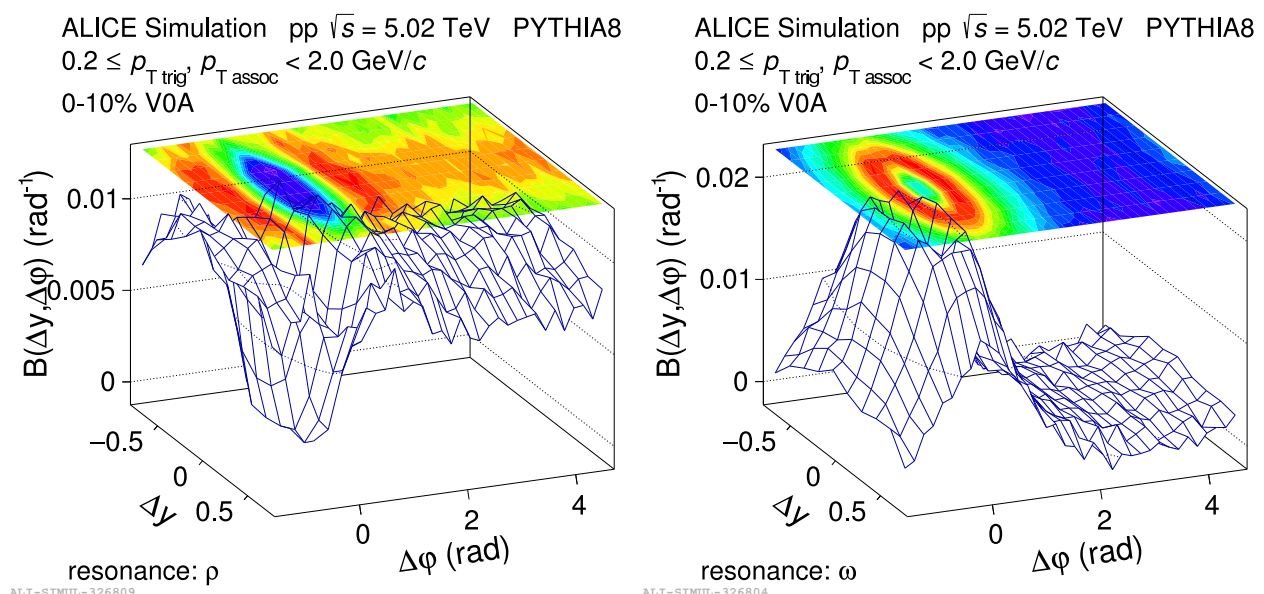

FiguRE 5.35: Balance function of $\rho$ (left) and $\omega$ (right) resonances in the PYTHIA8 model for $0-10 \%$ multiplicity class

As a next step, both resonances were removed by excluding the pairs of unlikesign pions that originated from the same mother with the pdg code of $\omega$ or $\rho$. The balance function of pions before and after the resonance removal is shown in Figure 5.36 for $0-10 \%$ multiplicity class since the effect is qualitatively similar for all multiplicities. It is seen that excluding $\omega$ and $\rho$ leads to a fully recovered peak on the near side of the distribution. This allows to conclude that in PYTHIA8 the near-side depletion in the balance function of pion pairs has a significant contribution originating from the decays of these two resonances. When compared with data, the depletion observed in PYTHIA8 is not as pronounced. This might indicate that either the relative contribution and yield of resonances is not the same in data and simulation or that additional effects could also contribute. One candidate for the latter is still the correlations originating from quantum statistics which are not part of the framework of PYTHIA8. At the same time, 
the away side of the distribution becomes less pronounced after the exclusion of resonances since it mostly comes from the $\rho$ balance function shape.
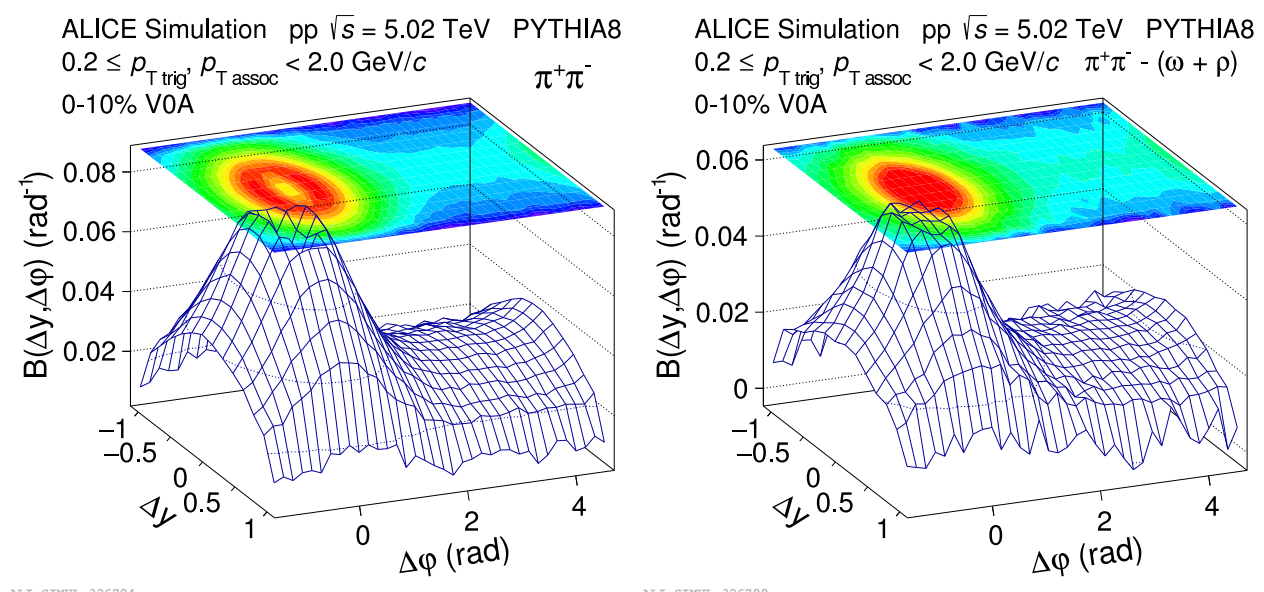

FIgURE 5.36: Pion balance function in the PYTHIA8 model before (left) and after (right) the removal of $\rho$ and $\omega$ resonances for $0-10 \%$ multiplicity class

The corresponding contribution of resonances for kaons is better presented in projections obtained according to the steps explained in Section 4.3 than with the two-dimensional distributions. Figure 5.37 shows the projection of the kaon balance function on $\Delta y$ (left) and $\Delta \varphi$ (right) at the generator level before and after the exclusion of $\phi$ resonance decaying into unlike-sign kaon pairs. Two distinct shapes can be seen in the total balance function of kaons: the peak and the shoulders. In order to further investigate these two distinct shapes, the balance function projection in both $\Delta y$ and $\Delta \varphi$ for kaons coming from the decay of the $\phi$ meson is also plotted in Figure 5.37. This distribution is represented by the red line. It is seen that it consists just of the near-side peak. Furthermore, the green line of Figure 5.37 represents the total kaon balance function excluding the $\phi$ meson contribution. The near-side peak of this distribution becomes much broader and shows a significant effect of the resonance decay on the shape of the balance function for kaon pairs. 

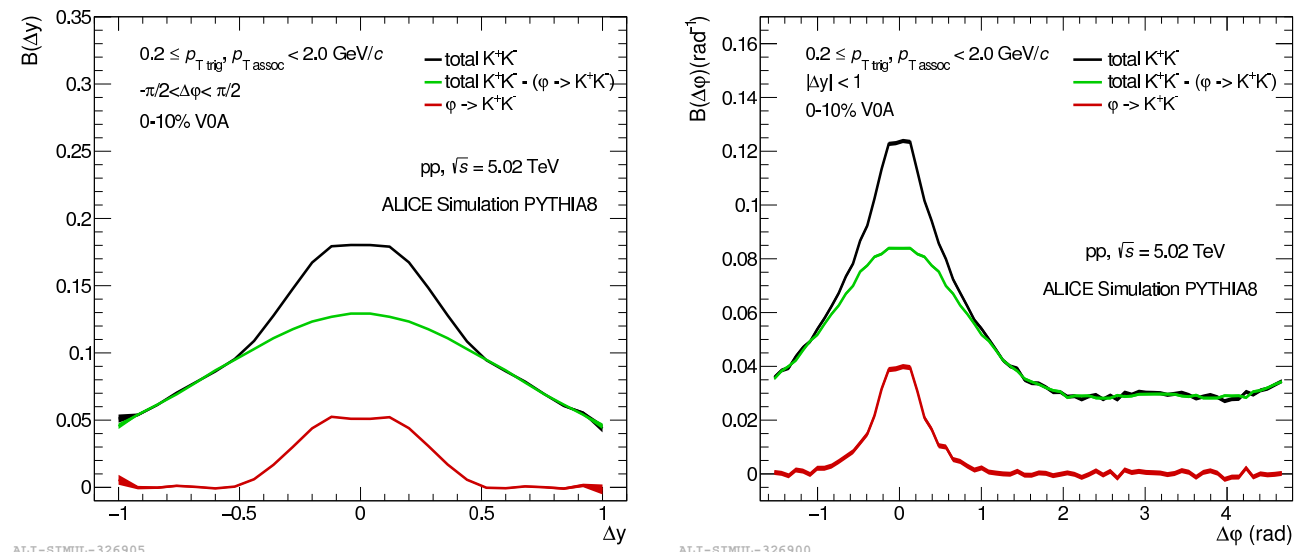

FiguRE 5.37: Kaon balance function projection on $\Delta \mathrm{y}$ (left) and $\Delta \varphi$ (right) in the PYTHIA8 model before (black) and after (green) the exclusion of $\phi$ resonance for $0-10 \%$ multiplicity class. $\phi$ balance function projection is shown in red

In addition, the effect of the resonance removal on the multiplicity-dependent narrowing of the balance function width was studied. Figures 5.38 and 5.39 show the multiplicity-dependent width in both dimensions for the pion and kaon balance function respectively at the generator level before and after the exclusion of the aforementioned resonances. It can be seen that the narrowing with increasing multiplicity is preserved even after the removal of the relevant contributions from resonances to both particle species. This means that the decay kinematics of resonances do not affect the multiplicity-dependent trend of the balance function width. However, it's interesting to point out a different change in the values of the width in these two cases: after the resonance removal the width for pions becomes narrower, while for kaons it becomes broader in every multiplicity bin. This has to do with the way resonances affect the shape of the two-dimensional distributions. In case of pions the underlying correlation of pion pairs is narrower with respect to a broader correlation coming from resonances, contrary to the case of kaon pairs, where the underlying correlation is broader than the one coming from the $\phi$ meson decay products. 

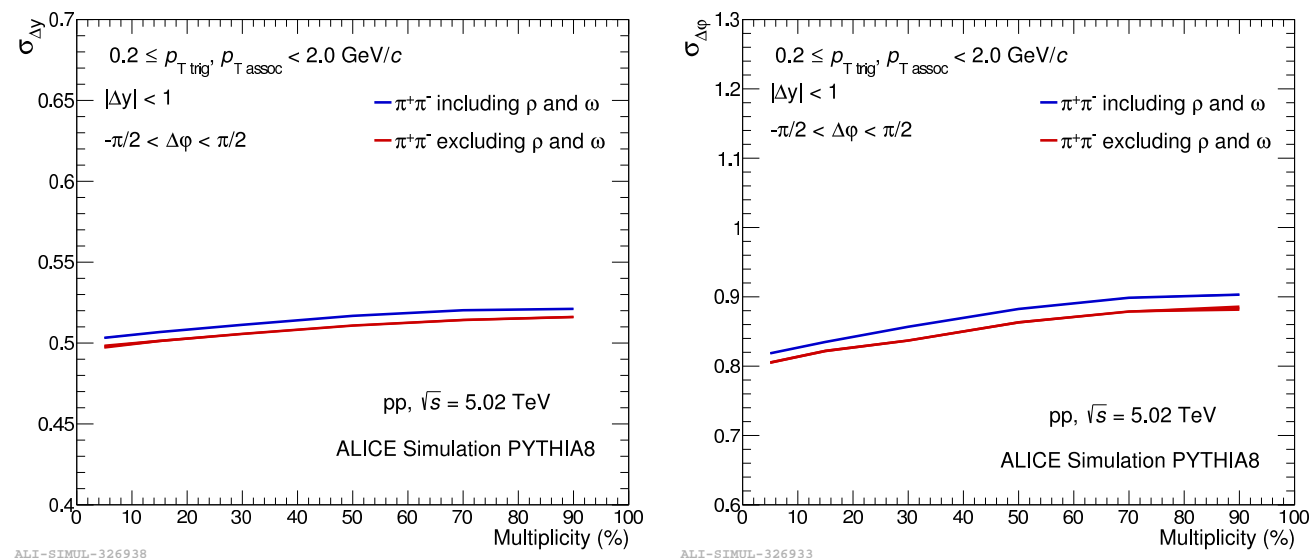

Figure 5.38: Pion balance function width $\sigma_{\Delta \mathrm{y}}$ (left) and $\sigma_{\Delta \varphi}$ (right) in the PYTHIA 8 model before (blue) and after (red) the exclusion of $\omega$ and $\rho$ resonances
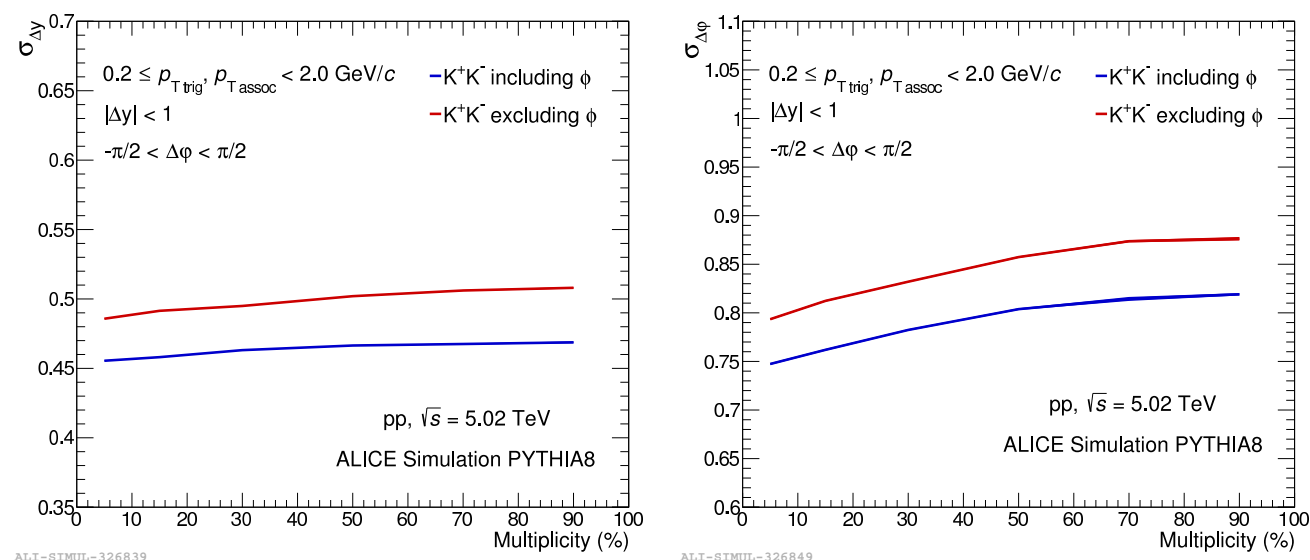

FIGURE 5.39: Kaon balance function width $\sigma_{\Delta \mathrm{y}}$ (left) and $\sigma_{\Delta \varphi}$ (right) in the PYTHIA 8 model before (blue) and after (red) the exclusion of $\phi$ resonance

\subsubsection{Balance function projections of pions and protons}

The balance function projections shown in this section are obtained from the two-dimensional distributions according to the steps described in Section 4.3. Figures 5.40 and 5.41 present the results of the multiplicity-dependent projections in $\Delta y$ and in $\Delta \varphi$ for pions, while Figures 5.42 and 5.43 - for protons. For every point, only statistical uncertainties are shown with the error bars. 

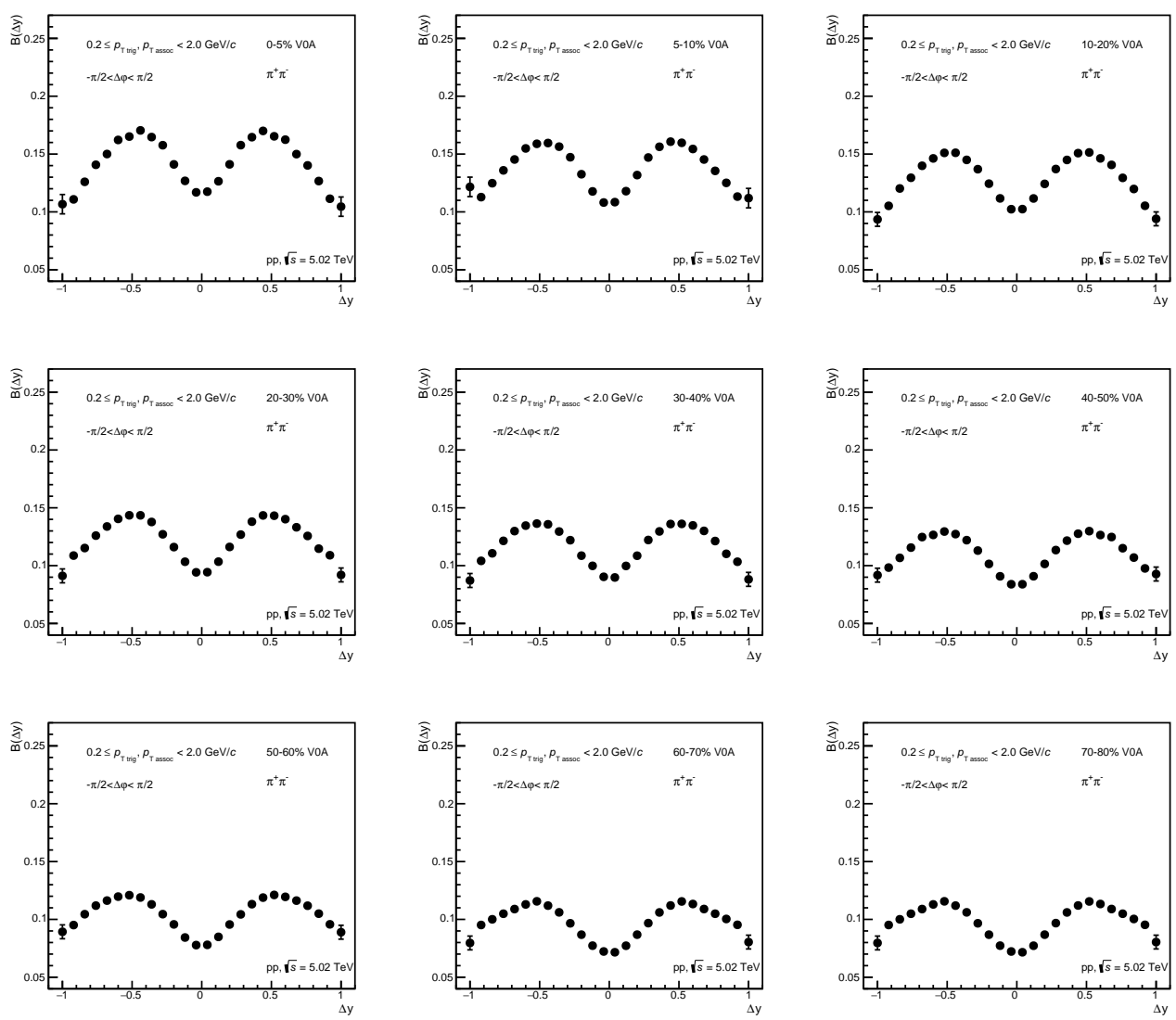

FIGURE 5.40: Balance function projections on $\Delta \mathrm{y}$ of pions in pp collisions at $\sqrt{\mathrm{s}}=5.02 \mathrm{TeV}$ as a function of multiplicity class 

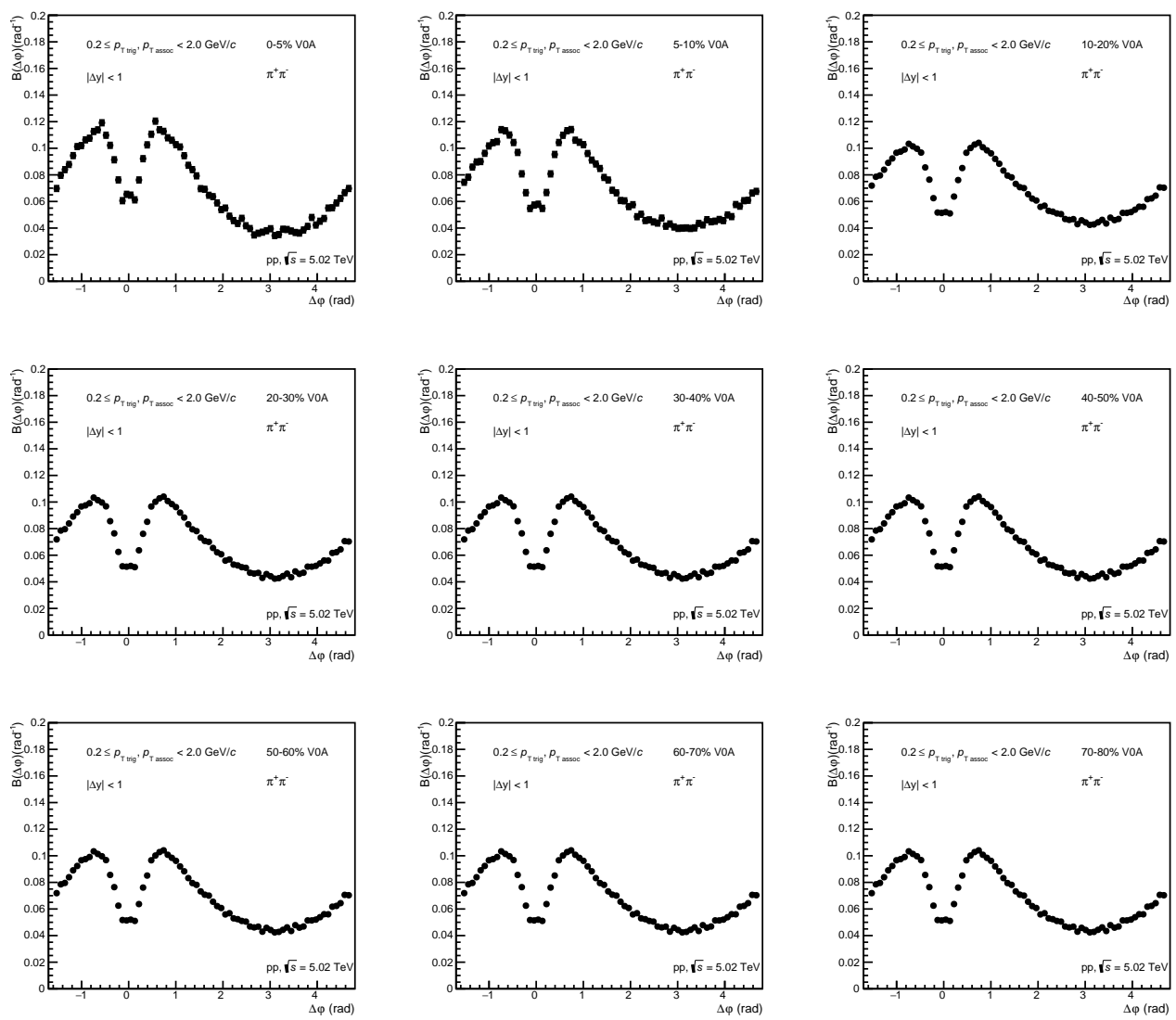

Figure 5.41: Balance function projections on $\Delta \varphi$ of pions in pp collisions at $\sqrt{\mathrm{s}}=5.02 \mathrm{TeV}$ as a function of multiplicity class 

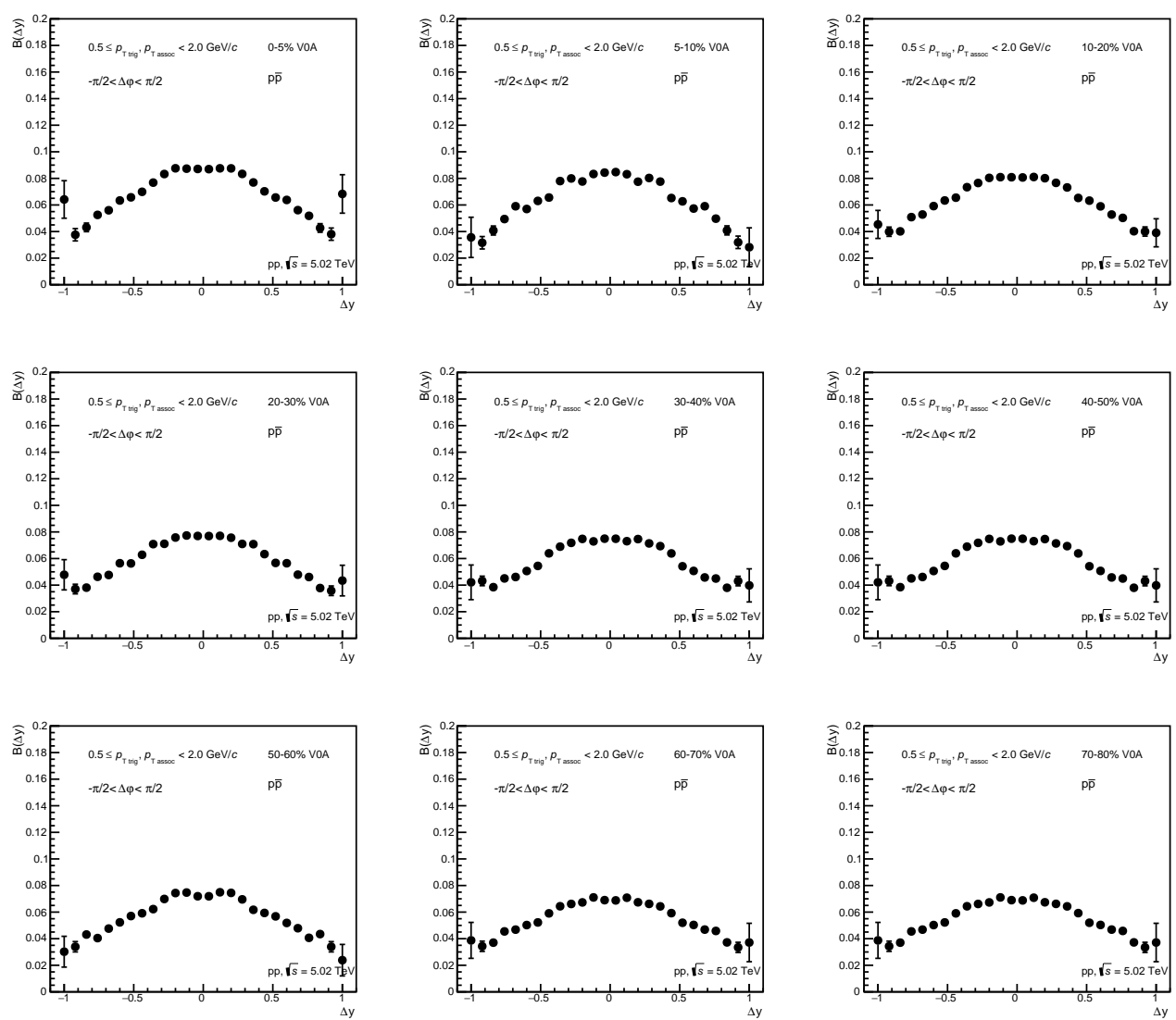

FIGURE 5.42: Balance function projections on $\Delta y$ of protons in pp collisions at $\sqrt{\mathrm{s}}=5.02 \mathrm{TeV}$ as a function of multiplicity class 

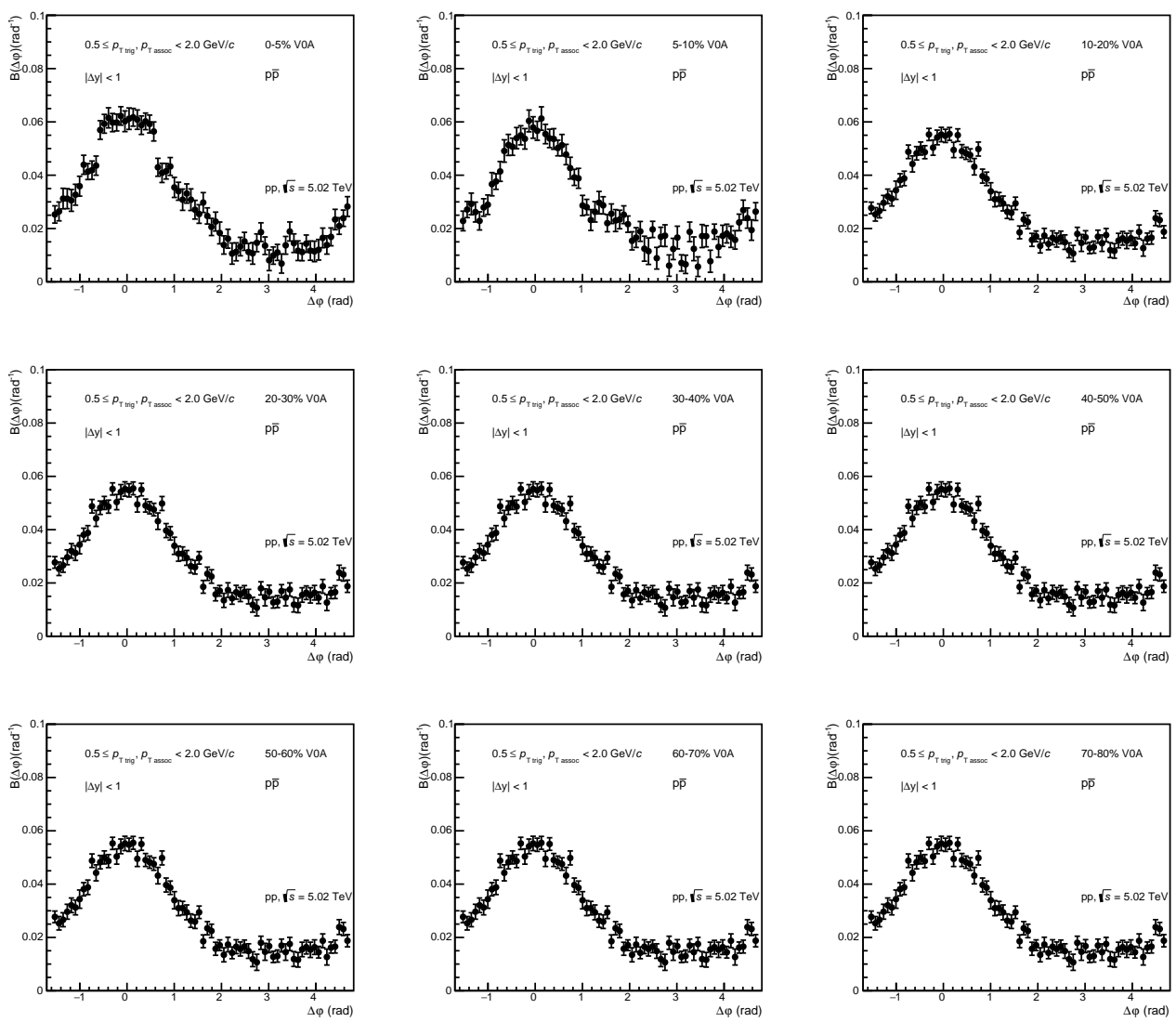

FIGURE 5.43: Balance function projections on $\Delta \varphi$ of protons in pp collisions at $\sqrt{\mathrm{s}}=5.02 \mathrm{TeV}$ as a function of multiplicity class

For an easier comparison, the projections for three selected multiplicity classes of $0-5 \%, 30-40 \%$ and $70-80 \%$ are shown together in Figure 5.44 for pions and Figure 5.45 for protons. In these results also the systematic uncertainties are indicated by the open boxes. A significant multiplicity dependence of the magnitude and the shape in $\Delta y$ as well as in $\Delta \varphi$ projections can be observed.

In the pion case, while the magnitude of the near side decreases with decreasing multiplicity, the away side magnitude becomes more pronounced. The depletion around $(\Delta y, \Delta \varphi)=(0,0)$, discussed in detail at the level of the two-dimensional distributions, becomes broader for lower multiplicities both in $\Delta y$ and $\Delta \varphi$.

In the case of protons the shape of the distributions does not change much with varying multiplicity.

A decrease of the balance function magnitude is observed with a decreasing multiplicity for both cases. This indicates a decreasing number of correlated balancing pairs in this category of events. Moreover, similarly to the case of charged particles analysis, it can be seen that the lower the multiplicity, the 
broader the near side of the balance function distribution becomes. This is caused by stronger charge correlations measured in high-multiplicity collisions. This behaviour is reflected in a clear way in how the width develops as a function of multiplicity class shown in the next section.
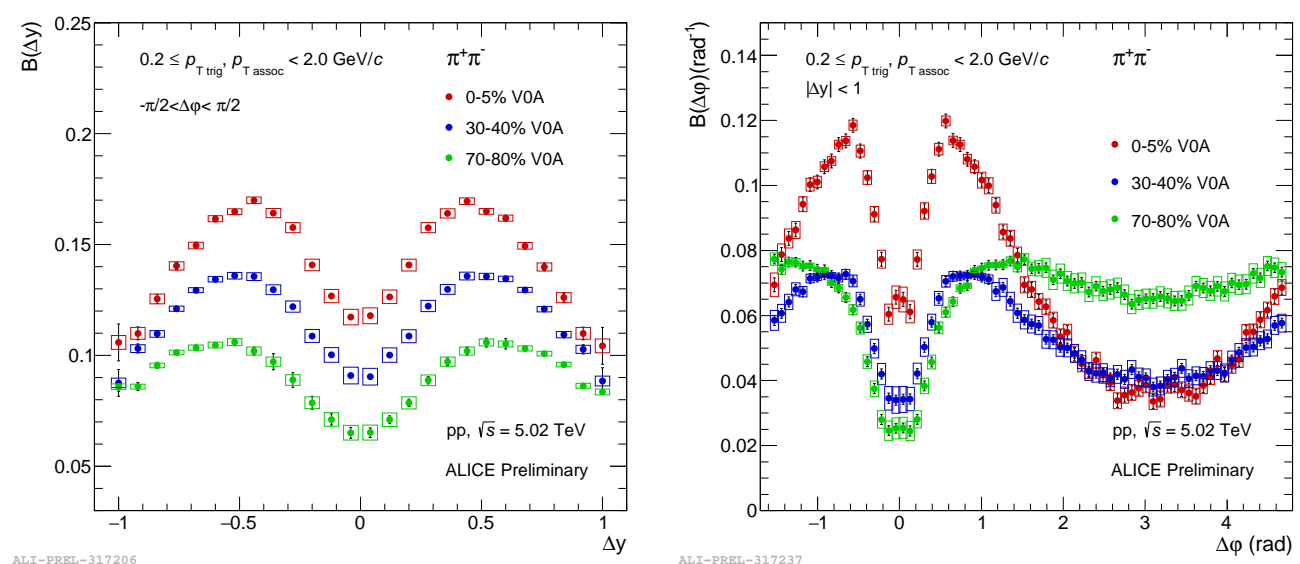

Figure 5.44: Balance function projections of pions in pp collisions at $\sqrt{\mathrm{s}}=$ $5.02 \mathrm{TeV}$ on $\Delta \mathrm{y}$ (left) and $\Delta \varphi$ (right) for $0-5 \%, 30-40 \%$ and $70-80 \%$ multiplicity classes
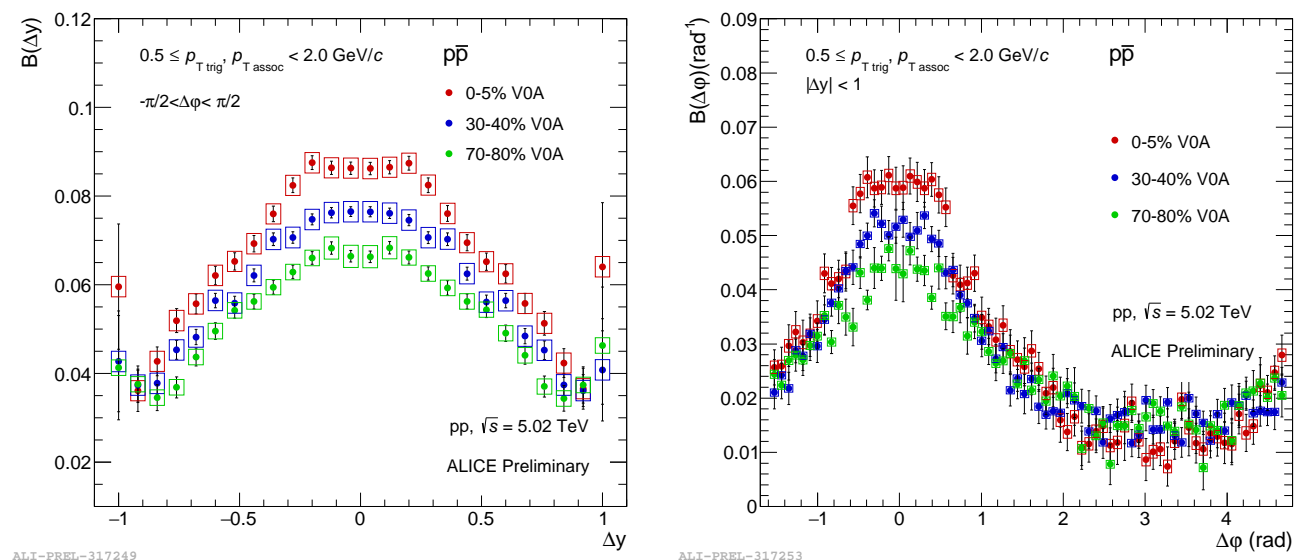

Figure 5.45: Balance function projections of protons in pp collisions at $\sqrt{\mathrm{s}}=$ $5.02 \mathrm{TeV}$ on $\Delta \mathrm{y}$ (left) and $\Delta \varphi$ (right) for $0-5 \%, 30-40 \%$ and $70-80 \%$ multiplicity classes

\subsubsection{Balance function width and yield of pions and protons}

The results of the multiplicity-dependent balance function width $\sigma_{\Delta y}$ and $\sigma_{\Delta \varphi}$ for pions and protons obtained according to the procedure explained in Section 4.3 are presented together with the results for charged particles (see Section 5.1.1) in Figure 5.46. Similarly, results of the balance function yield as a function of multiplicity class are shown in Figure 5.47. For every point the statistical 
uncertainties are shown as the error bars, while systematic uncertainties are indicated by the open boxes.

As seen in Figure 5.46, the values of the width in both dimensions for pions are slightly higher with respect to the charged particles in every multiplicity class. This is caused by a more pronounced depletion around $(\Delta \mathrm{y}, \Delta \varphi)=(0,0)$ in the balance function of pions compared with the one of charged particles. However, the overall multiplicity-dependent trend is almost the same in these two cases which is due to the fact that pions form the majority of charged particle sample, hence reflect almost the same behaviour.

The balance function width values for protons are smaller than for pions. This is expected, first of all, due to the inverse dependence of the spatial separation between balancing charges on the mass of the particle explained in detail in Chapter 2. Moreover, this is expected according to the assumptions related to the stronger influence of the "radial flow-like" effects on the heavier particles discussed in Section 5.2.

A very interesting observation comes with the multiplicity dependence of the width for both particle species. While a decreasing trend with increasing multiplicity is observed for pions, for protons the dependence is rather flat: the linear fit performed in both cases shows that the slope for pions is more than $5 \sigma$ away from 0 , but for protons it's compatible with 0 within $2 \sigma$. Therefore, these results are not in agreement with the expectation of observing more pronounced narrowing with increasing multiplicity for protons than for pions if "radial flow-like" effects are the origin behind the multiplicity-dependent narrowing. This might suggest that some other effects that are not connected to collectivity might drive such behaviour in pp collisions.

To understand if this is true not only for pp collisions, but for small systems in general, a comparison of the presented results to the results in $\mathrm{p}-\mathrm{Pb}$ collisions was performed and can be found in Section 5.3. 

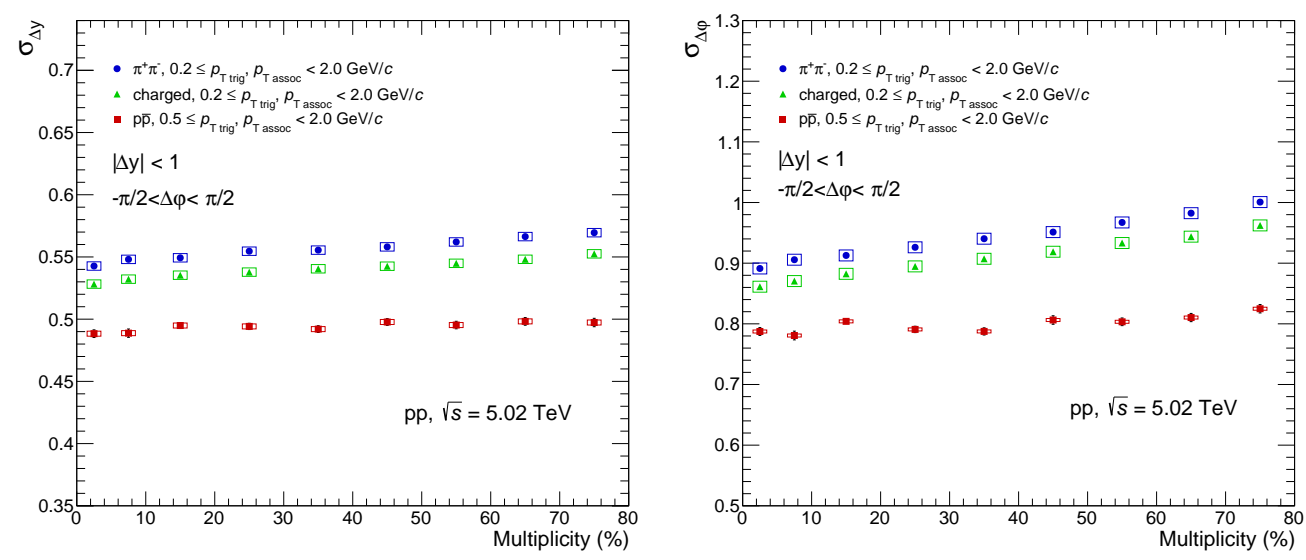

FiguRE 5.46: Balance function width $\sigma_{\Delta y}\left(\sigma_{\Delta \eta}\right.$ for charged particles $)$ on the left and $\sigma_{\Delta \varphi}$ on the right for charged particles, pions and protons as a function of multiplicity class

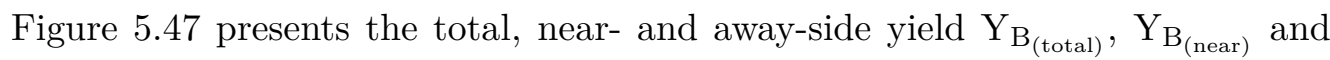
$\mathrm{Y}_{\mathrm{B}_{(\text {away }}}$ of pions and protons pairs in comparison to the similar values measured for pairs of charged particles. These three results allow to obtain the number of correlated unlike-sign charge pairs in different parts of $(\Delta y, \Delta \varphi)$ space. The values of the yield mentioned in the text below are given in terms of relative values, defined for the total calculated yield as $\mathrm{Y}_{\mathrm{r}_{i_{\text {total }}}}=\frac{\mathrm{Y}_{\mathrm{i}_{\text {total }}}}{\mathrm{Y}_{\max }} \cdot 100 \%$, where $\mathrm{Y}_{\max }=1$ is the maximum possible total yield, while i refers to a particle type.

It can be seen that the values of the yield in all three cases for pions are slightly lower than the values for charged particles in the same multiplicity class. Such a decrease is due to smaller amount of correlation measured overall in case of the pion analysis with respect to all charged particles. Similarly as in the case of the width results, the multiplicity-dependent yield results for pions are similar to the one of charged particles.

The total yield of pions decreases slightly with decreasing multiplicity. This observation might be caused by the fact that a part of the correlated pion pairs in lower multiplicity classes, where the balance function becomes much broader, falls outside the detector acceptance range and is not measured. In the results of the near-side yield it is seen that in the highest multiplicity largest fraction of the total measured correlation of pion pairs is located at the near side, while in the lowest multiplicity class the fraction is rather equal on the near and away sides.

In all three results lower yield for protons is observed with respect to pions. This indicates smaller amount of correlated proton pairs produced in the collision with respect to pions. From the values of the total yield it can be seen that while around $42-45 \%$ of all produced correlated unlike-sign pion pairs are reconstructed 
in a given acceptance range of $|\mathrm{y}|<0.5$, for protons it's only 17-20\%. Contrary to the pion case, the total yield of protons shows no significant multiplicity dependence. The similar trend is observed in the results of the near and awayside yields.
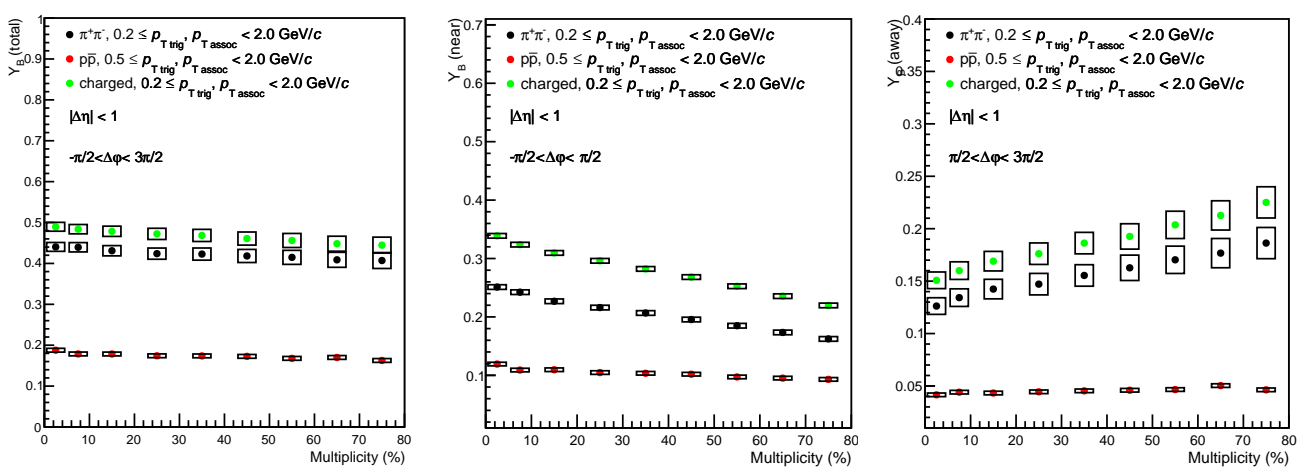

Figure 5.47: Total (left), near-side (middle) and away-side (right) yields of charged particle, pion and proton balance function in pp collisions at $\sqrt{\mathrm{s}}=$ $5.02 \mathrm{TeV}$ as a function of multiplicity class

\subsubsection{Model comparison}

The reported results of the balance function for pion and proton pairs were compared with the prediction from the PYTHIA8 Monte Carlo model. The V0A estimator was used for the multiplicity estimation and the same kinematic constrains as in the data analysis were applied. The analysis was performed over primaries at the generated level and particle species were selected using the Monte Carlo particle data group (pdg) code.

Two different tunes were used in the model: with and without the so called color reconnection mechanism, as it was also done in the previous studies of charged particles in pp collisions at $\sqrt{\mathrm{s}}=7 \mathrm{TeV}$ [111]. This mechanism is based on the string fragmentation model, i.e. on the calculation of the probability to connect partons by color lines, or strings. The result expected in such a model, as it is implemented in PYTHIA8, is shown schematically in Figure 5.48. In Figure 5.48 (a) a hard gluon-gluon subcollision is happening where the gluons are color-connected to the projectile and target remnants. Next, as presented in Figure 5.48 (b), a second hard scattering might occur. This would naively be expected to give two new strings connected to the remnants. In Figure 5.48 (c) the gluons are finally color reconnected, so that the total string length becomes as short as possible [113]. Therefore, the fragmentation of two independent hard scatterings are dependent on each other which will lead to a rise of $\left\langle\mathrm{p}_{\mathrm{T}}\right\rangle$ with multiplicity [83]. In addition, since two partons from independent hard 
scatterings at mid-rapidity can color reconnect and make a large transverse boost, it will result in an effective common boost of the final string fragments - hadrons. Therefore, such boost effect is similar to the effect of radial flow on hadrons in hydrodynamics (see Chapter 1 for more details).

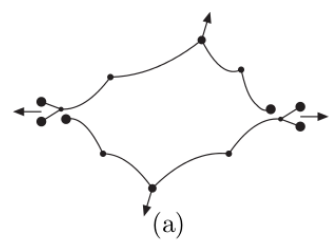

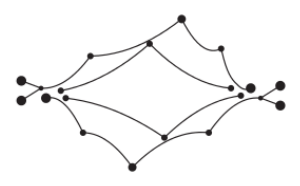

(b)

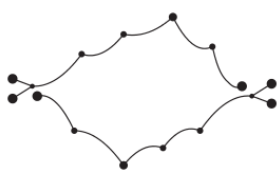

(c)

FIGURE 5.48: Schematic representation of color reconnection in the string fragmentation model: the gluons are color connected to the projectile and target remnants (a), the second hard scattering (b), color reconnected string(c) [113]

The results of the two-dimensional pion balance function for $0-10 \%, 10-20 \%, 20$ 40\%, 40-60\%, 60-80\% and 80-100\% multiplicity classes obtained in PYTHIA8 with the color reconnection are shown in Figure 5.49, while without the color reconnection - in Figure 5.50. It can be seen that the magnitude of the distributions on the near side becomes more pronounced and the distribution becomes narrower for higher multiplicity. On the away side the opposite effect is observed with the magnitude increasing towards lower multiplicity. The depletion around $(\Delta \mathrm{y}, \Delta \varphi)=(0,0)$ is present in all multiplicity bins but becomes more pronounced for the lower ones.

On the contrary, in case of the results without color reconnection, no significant increase of the magnitude is observed on the near side towards increasing multiplicity. Instead, the away side of the distribution becomes more and more dominating with decreasing multiplicity. The near-side dip is observed but it does not seem to have a significant multiplicity dependence. For the highest multiplicity, magnitude of the measured distribution is around two times higher for the color reconnection tune in comparison to the one without color reconnection. 

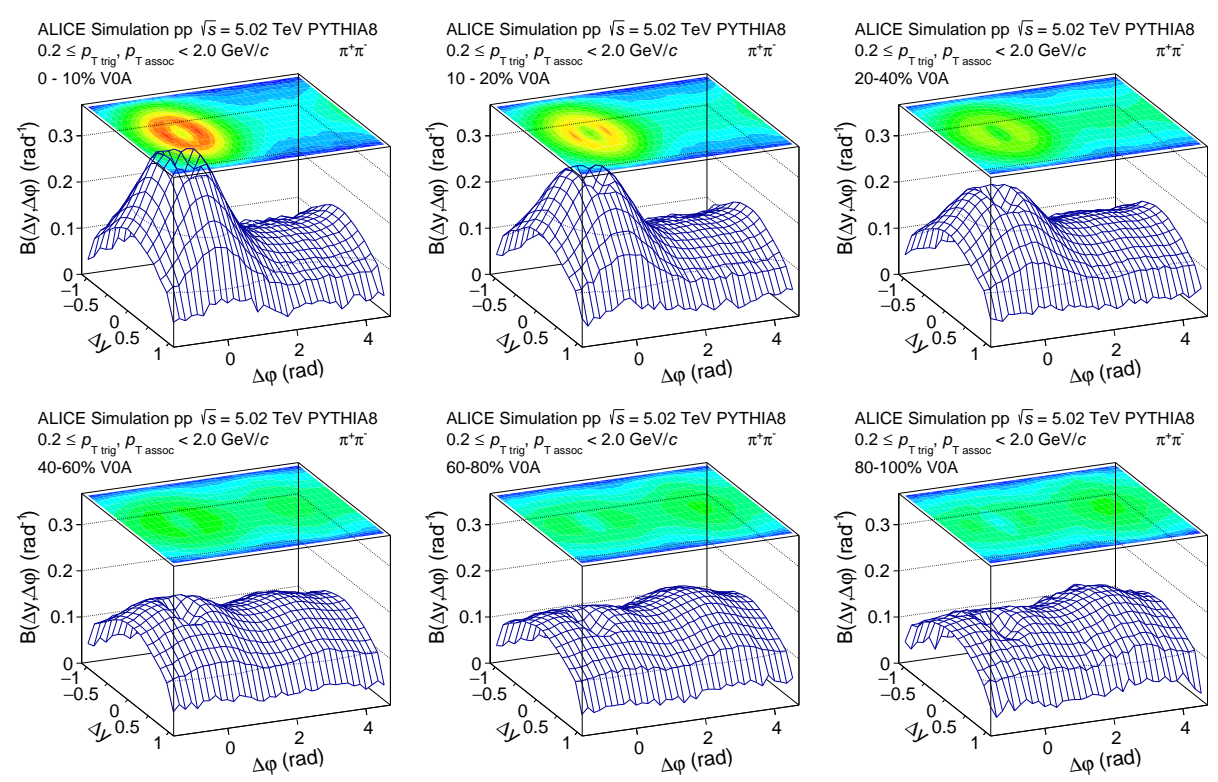

Figure 5.49: Two-dimensional balance function of pion pairs in PYTHIA8 with the color reconnection as a function of multiplicity
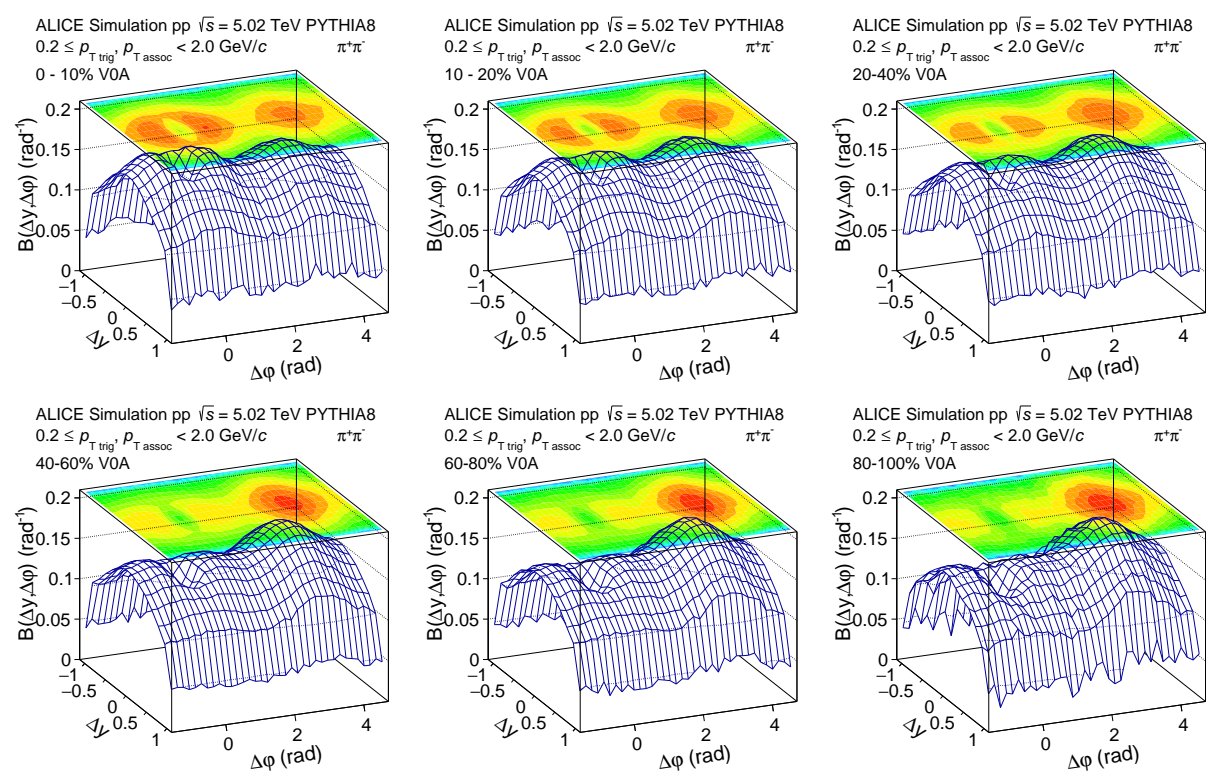

FIGURE 5.50: Two-dimensional balance function of pion pairs in PYTHIA8 without the color reconnection as a function of multiplicity

For protons similar results are shown in Figures 5.51 and 5.52. A pronounced near-side peak is observed in both figures with magnitudes increasing for higher multiplicities. It is interesting to see that the shape of the distributions does not change much overall in the first case with respect to the second case. However, the magnitude is two times higher in the highest multiplicity class in the tune with color reconnection similarly as for pions. 

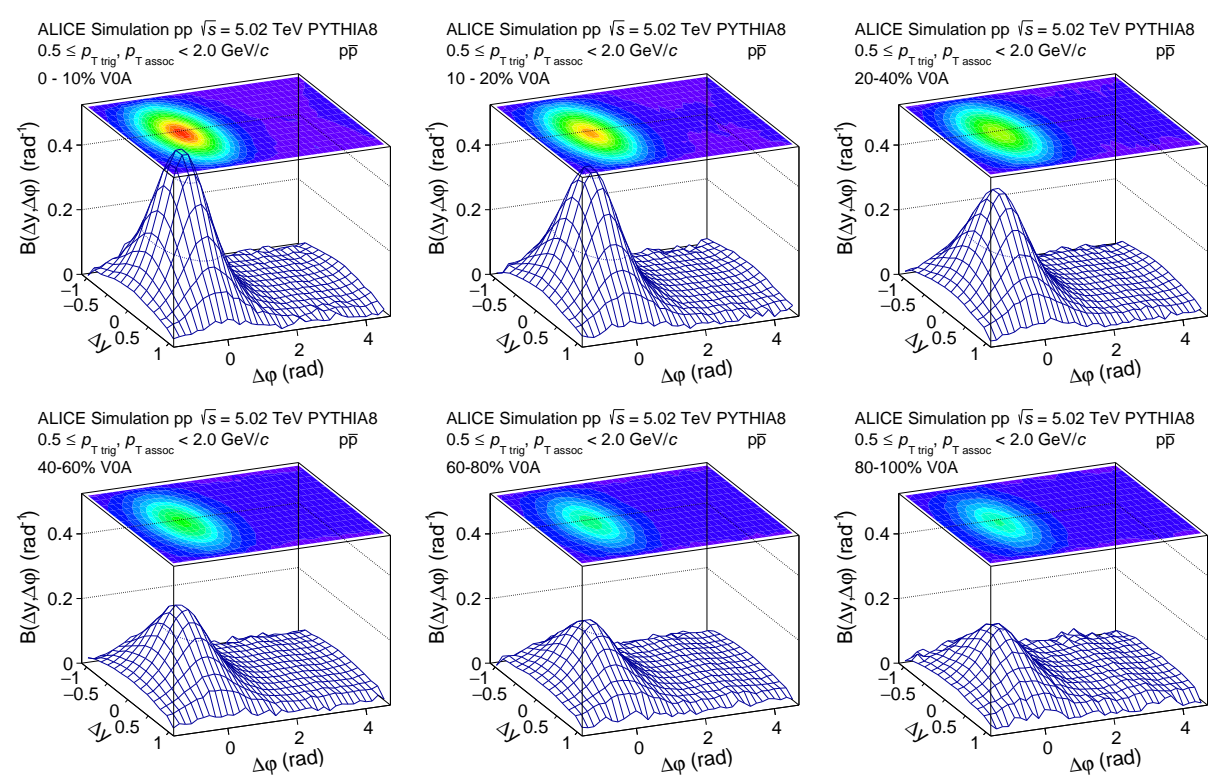

FiguRE 5.51: Two-dimensional balance function of proton pairs in PYTHIA8 with the color reconnection as a function of multiplicity
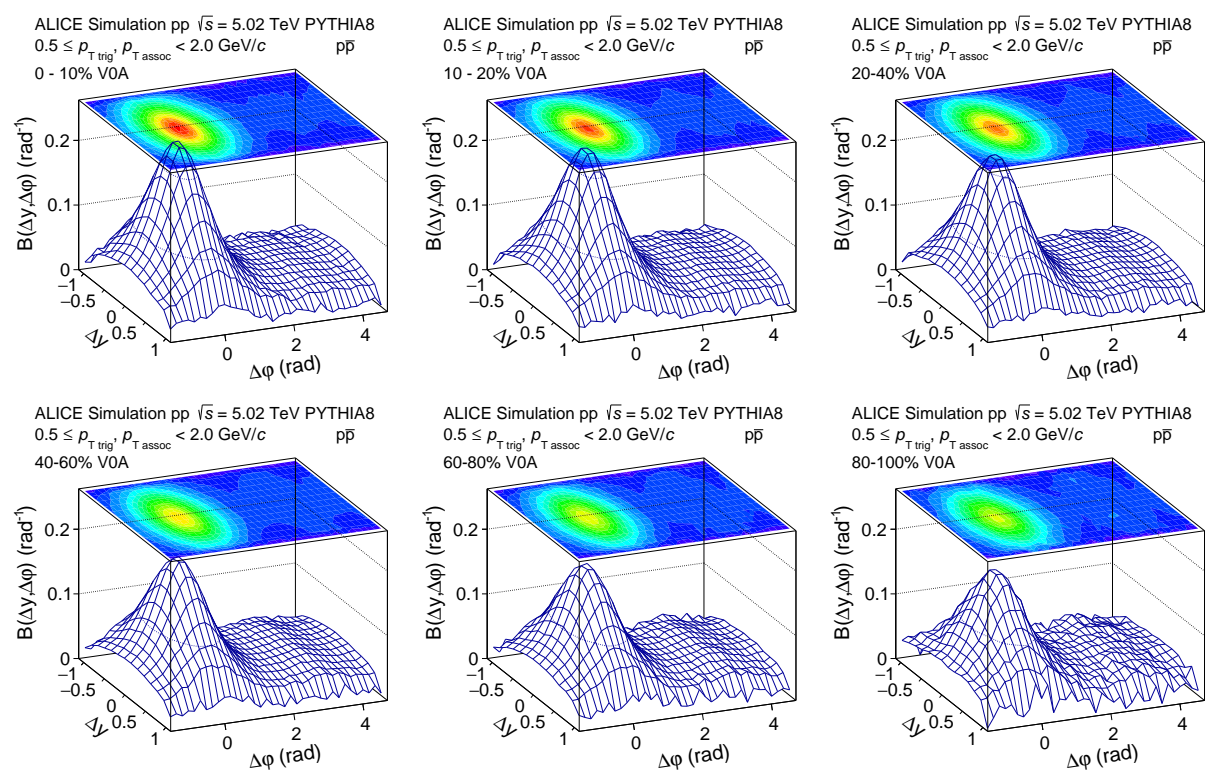

FIGURE 5.52: Two-dimensional balance function of proton pairs in PYTHIA8 without the color reconnection as a function of multiplicity

For the purpose of better comparison, the balance function projections for pions in $\Delta y$ are shown in Figure 5.53, while in $\Delta \varphi$ - in Figure 5.54 for both model tunes together with the data points. In Figure 5.53 , it is seen that for the tune with color reconnection the data points are reproduced quite well both quantitatively and qualitatively in every multiplicity class with the exception of the near-side dip. This is expected due to the fact that quantum statistics correlations are 
not included at the level of the simulation, while they explain a large fraction of the depletion in the data. Therefore, it is much more pronounced in the data in comparison to the model results. It is important to point out that in the previous analysis of charged particles balance function, discussed in Section 5.1.1, it was shown that the removal of the depletion, using specific requirements on the transverse momentum in the data, did not change the multiplicity-dependent trend of the balance function width. Considering this, the presence of the nearside depletion in pion balance function in this analysis is not expected to bias the multiplicity dependence of its width, presented later in this section. As for the away side, its magnitude in Figure 5.54 seems to be overestimated in both model analyses. For the case of the tune with no color reconnection the magnitude and the widths of the distributions are not very well reproduced.
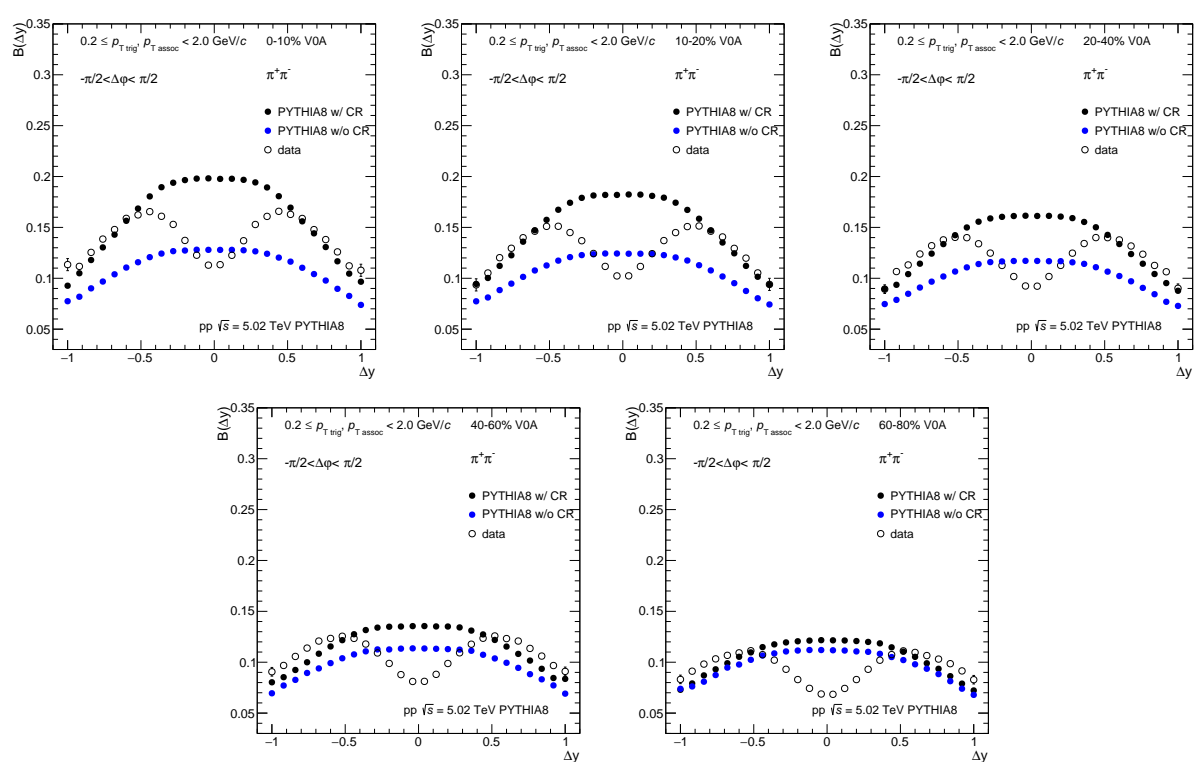

FIGURE 5.53: Balance function projections for pions on $\Delta y$ in PYTHIA 8 with and without the color reconnection compared to the data results as a function of multiplicity 

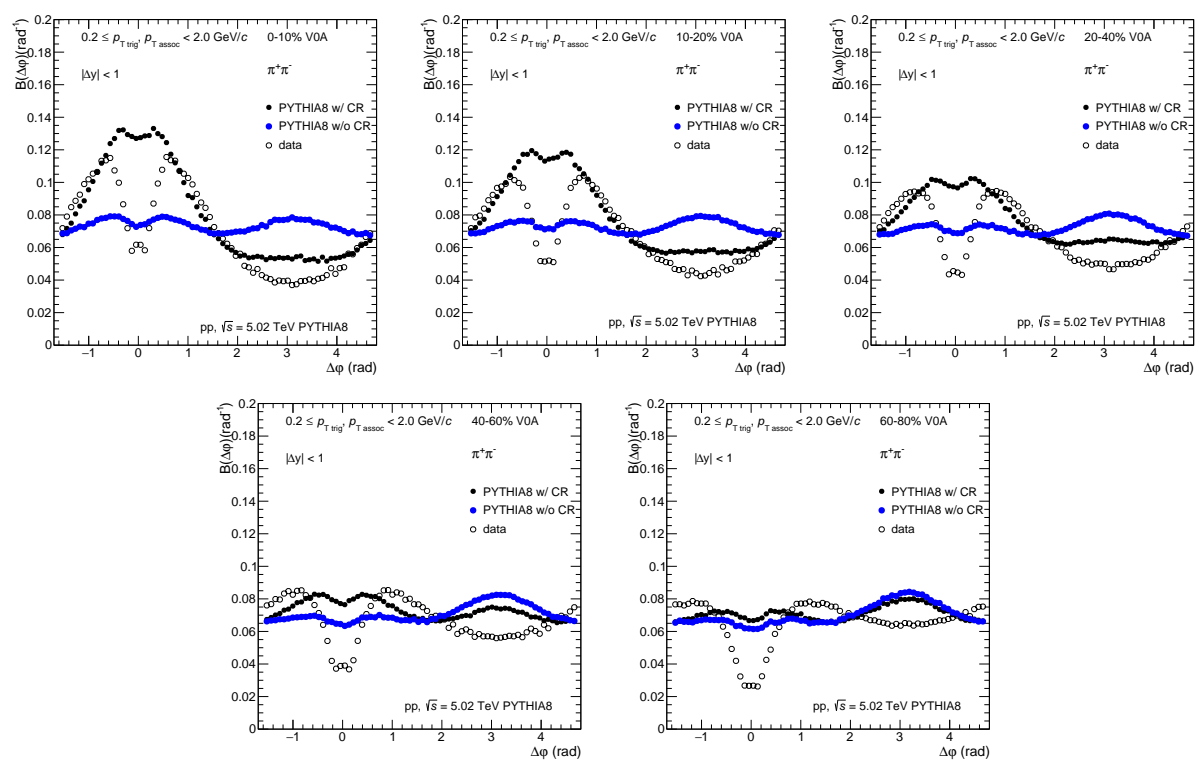

FiguRE 5.54: Balance function projections for pions on $\Delta \varphi$ in PYTHIA8 with and without the color reconnection compared to the data results as a function of multiplicity

Figures 5.55 and 5.56 present similar results for protons. In both dimensions, the magnitudes and the shape measured in the data are reproduced by neither of the tunes. The absence of short-range correlations in the simulation which originate, for instance, from Fermi-Dirac quantum statistics for identical protons, is not expected to cause a large discrepancy with respect to data results due to rather small contribution of these effects in the data at the level of the balance function distribution, i.e. the unlike-sign correlation for protons was observed to prevail over the like-sign correlation in previous data analyses. In addition, the transverse momentum threshold, i.e. $\mathrm{p}_{\mathrm{T}}>0.5 \mathrm{GeV} / \mathrm{c}$, used to select trigger and associated particles, is expected to significantly reduce the influence of this component. However, to confirm such expectations it would be useful to study the direct effect of the removal of short-range correlations on the balance function of identified hadrons in the data and the related systematic uncertainty in the future. 

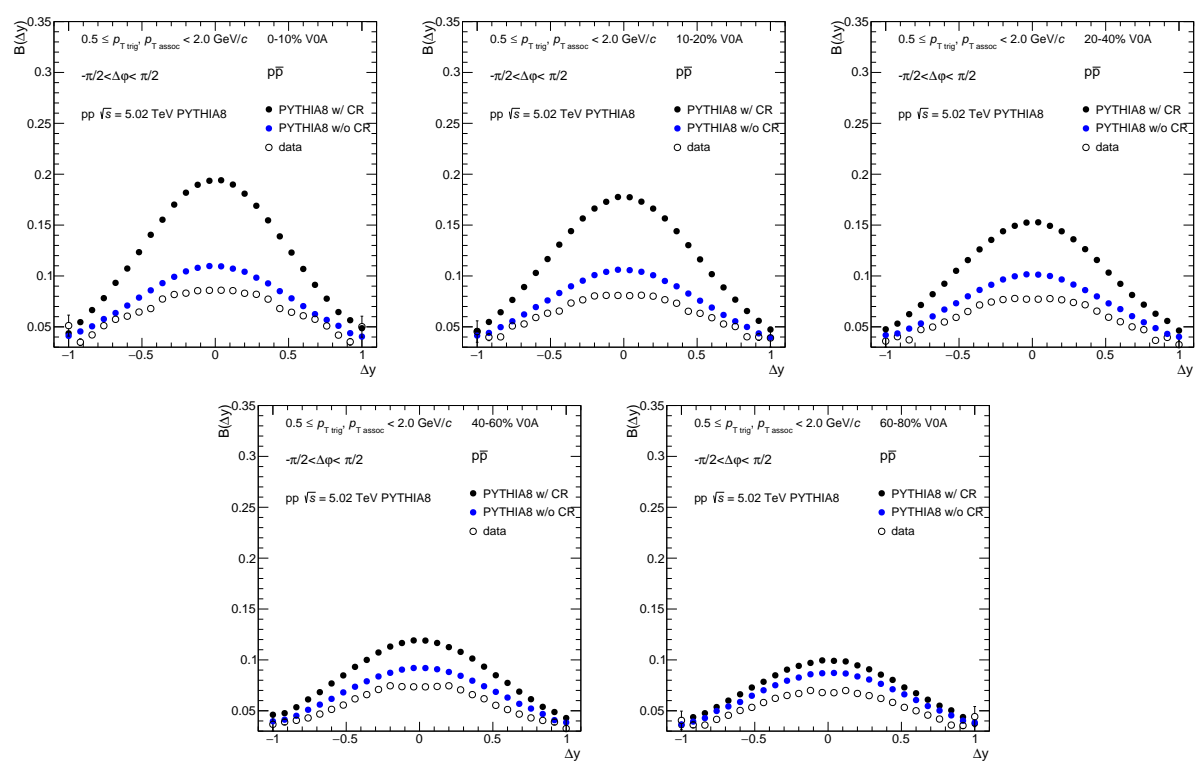

FiguRE 5.55: Balance function projections for protons on $\Delta y$ in PYTHIA8 with and without the color reconnection compared to the data results as a function of multiplicity
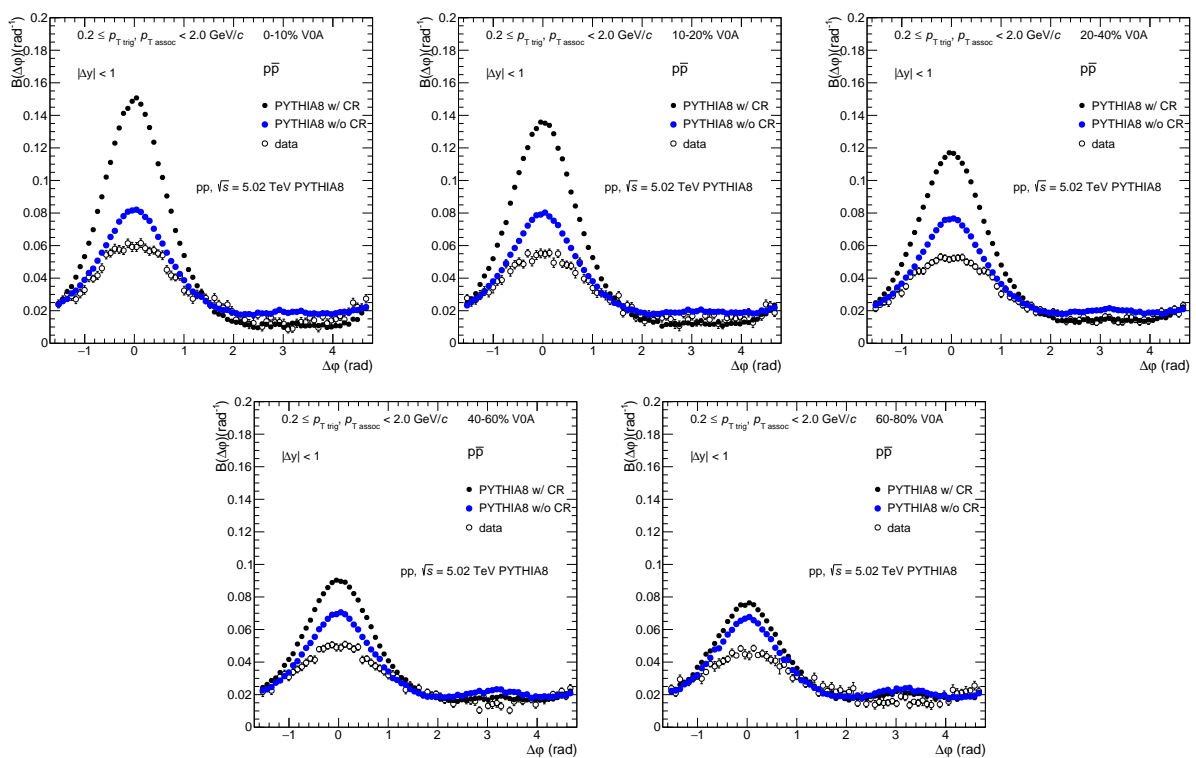

FIGURE 5.56: Balance function projections for protons on $\Delta \varphi$ in PYTHIA8 with and without the color reconnection compared to the data results as a function of multiplicity

Figures 5.57 and 5.58 show the data results of pion and proton balance function width in $\Delta y$ and $\Delta \varphi$ compared with both PYTHIA8 calculations. It can be seen that the tune with the color reconnection expects a significant multiplicity dependence of the balance function width in both dimensions that is more pronounced for protons. This observation is in agreement with previous assumption 
made in Section 5.2 based on the fact that heavier particles should be more sensitive to "radial flow-like" effects. However, since data for protons show no trend as a function of multiplicity, the model prediction is in contradiction with it. This suggests that color reconnection is disfavoured by data as an origin of the narrowing. Together with the results from Section 5.2 this leads to a conclusion that "radial flow-like" effects might not be the main reason behind the narrowing of the balance function width with increasing multiplicity in pp collisions.

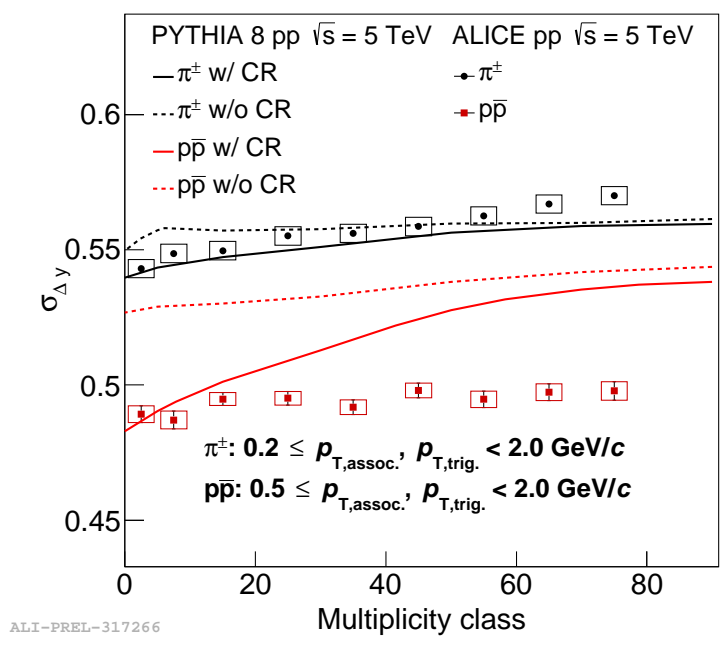

FIGURE 5.57: Balance function width $\sigma_{\Delta \mathrm{y}}$ as a function of multiplicity class for pions and protons compared to PYTHIA8 prediction with and without color reconnection in pp collisions at $\sqrt{\mathrm{s}}=5.02 \mathrm{TeV}$

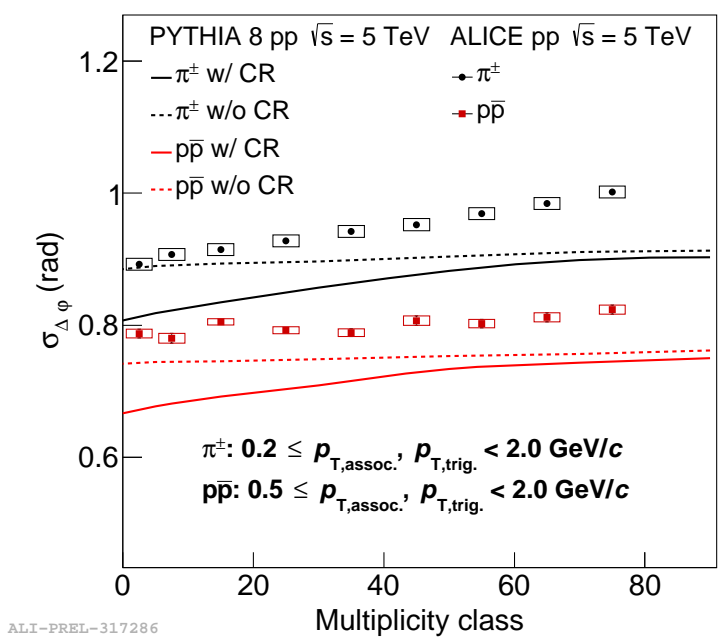

FIGURE 5.58: Balance function width $\sigma_{\Delta \varphi}$ as a function of multiplicity class for pions and protons compared to PYTHIA8 prediction with and without color reconnection in pp collisions at $\sqrt{\mathrm{s}}=5.02 \mathrm{TeV}$ 


\subsection{Comparison of the balance function of charged and identified particles in $\mathrm{pp}$ and $\mathrm{p}-\mathrm{Pb}$ collisions at $\sqrt{\mathrm{s}}=5.02 \mathrm{TeV}$}

The results of the balance function of charged particles and identified hadrons in pp collisions were compared to the results in another small system, namely $\mathrm{p}-\mathrm{Pb}$ collisions, at the same energy. This comparison allows to check if the multiplicity-dependent trend of the balance function width for different particle species is similar across different small systems. Since multiplicity classes do not mean the same number of particles due to the different system size of pp system with respect to $\mathrm{p}-\mathrm{Pb}$ system, the comparison was done as a function of averaged charged particles number reconstructed in $|\eta|<0.8$ and $\mathrm{p}_{\mathrm{T}}>0.2 \mathrm{GeV} / \mathrm{c}$.

From the results shown in Figure 5.59 for $\sigma_{\Delta y}$ and Figure 5.60 for $\sigma_{\Delta \varphi}$ it can be seen that multiplicity dependence of the balance function width is similar for charged particles, pions and protons in both systems. It can be also seen that the values of the width at the same multiplicities in two systems are quite close to each other. This leads to the conclusion that the mechanism that drives the multiplicity-dependent narrowing of the balance function width not only for charged particles, but also identified particles in small systems should be the same.

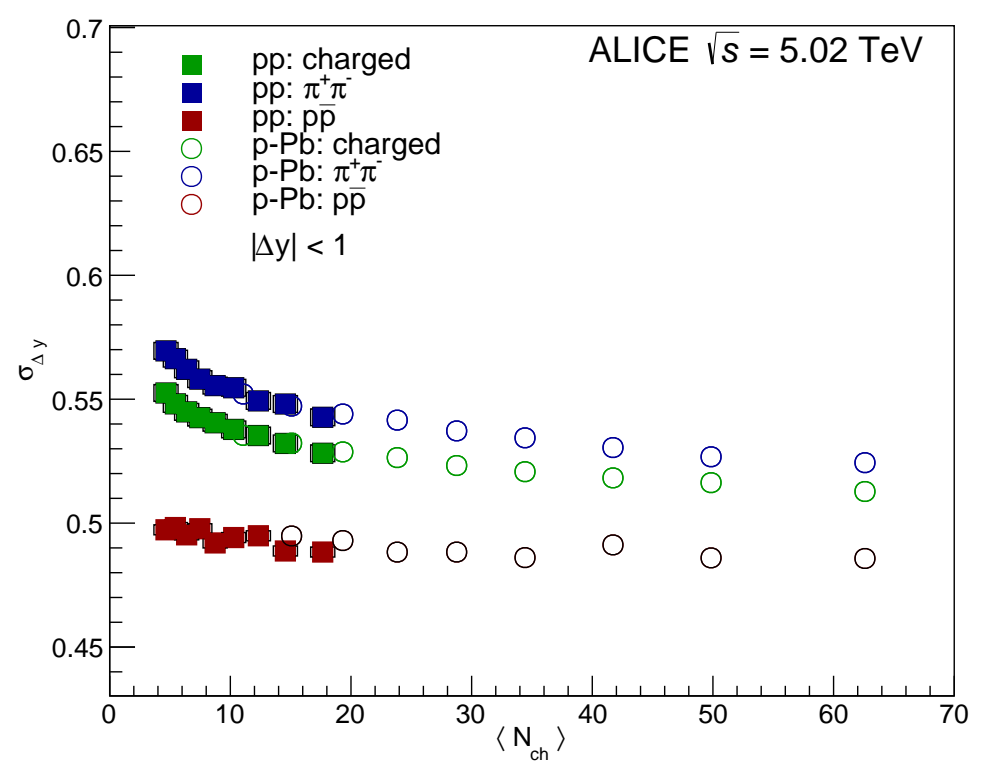

FIGURE 5.59: Balance function width $\sigma_{\Delta y}$ of different particle species in pp and $\mathrm{p}-\mathrm{Pb}$ collisions at $\sqrt{\mathrm{s}}=5.02 \mathrm{TeV}$ as a function of multiplicity 


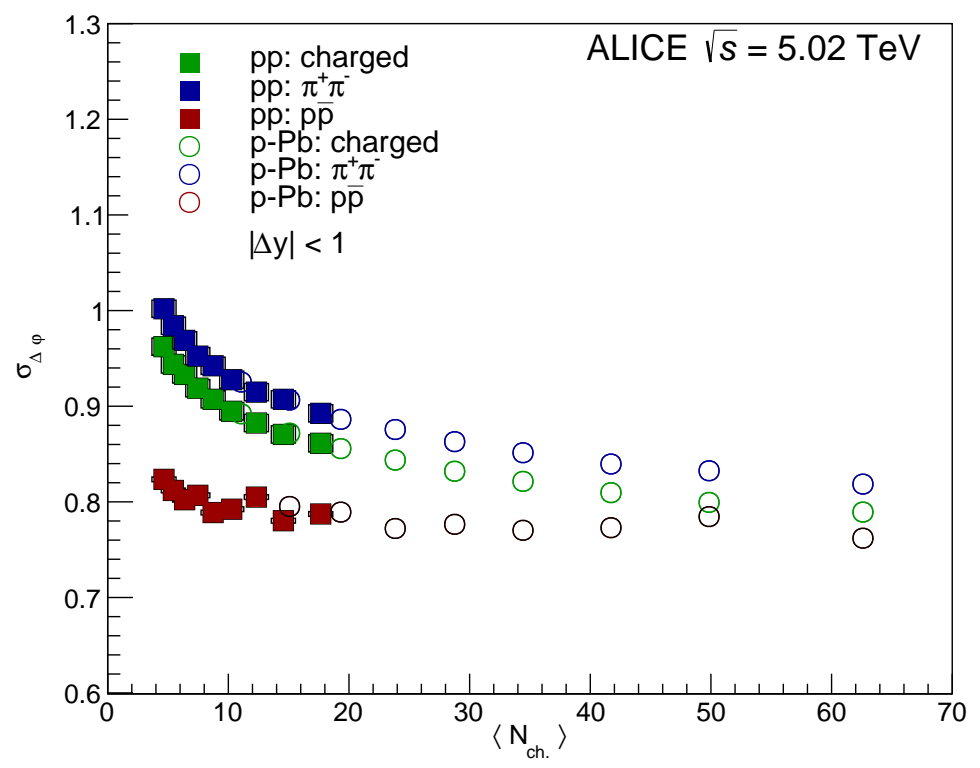

FIGURE 5.60: Balance function width $\sigma_{\Delta \varphi}$ of different particle species in pp and $\mathrm{p}-\mathrm{Pb}$ collisions at $\sqrt{\mathrm{s}}=5.02 \mathrm{TeV}$ as a function of multiplicity 


\section{Summary}

The main purpose of the heavy-ion physics program of the ALICE experiment at the LHC is to study the properties of the quark-gluon plasma (QGP), a deconfined medium made of quarks and gluons created in collisions between ultra-relativistic nuclei. Such study allows probing the behaviour of strongly interacting matter at high energy densities and temperatures. Moreover, since the QGP is believed to have existed during few microseconds after the Big Bang, it allows investigating the conditions present in the early Universe.

A very short lifetime of the QGP state that quickly expands and cools down does not make it accessible for the direct experimental measurements, therefore its properties are studied through the final state detected particles that can provide indirect evidences for the QGP formation. Previous studies of various experimental observables allowed to conclude that the medium created in heavy-ion collisions behaves as a nearly perfect liquid, i.e. its expansion is successfully described by equations of relativistic viscous hydrodynamics incorporating very low viscosity values. According to this description, the created pressure gradients lead to the collective motion of all produced particles from the reaction zone outwards with collective average transverse velocity field called radial flow. In addition, in non-central collisions the anisotropic flow effect is present, when the spatial anisotropy of the overlap region between colliding nuclei is transformed into the final state anisotropy in the momentum space. One of most prominent experimental reflections of the hydrodynamic expansion of a QGP is seen in two-particle angular correlations studied as a function of the relative pseudorapidity $\Delta \eta$ and azimuthal angle $\Delta \varphi$ of the particle pair. The enhancement in the amount of particle pairs located around $\Delta \varphi \sim 0$ and extending along all $\Delta \eta$ values, referred to as the near-side ridge, is attributed to long-range correlations originated from anisotropic flow.

As a reference for heavy-ion case, i.e. in order to disentangle effects connected to the formation of a hot and dense QGP medium from cold nuclear effects, proton-nucleus or proton-proton collisions have been used, where QGP was not expected to form due to the smaller size of these systems. Surprisingly, recent 
measurements performed by many experiments at RHIC and the LHC revealed the presence of the near-side ridge also in high-multiplicity proton-nucleus and proton-proton collisions. This discovery was followed by many other studies in small systems where similar effects attributed to collectivity in heavy-ion collisions were found, such as non-zero results of flow coefficients computed using not only two-, but also multi-particle correlations, the negative sign of four-particle cumulant, the hardening of particle $\mathrm{p}_{\mathrm{T}}$ spectra in high- compared with lowmultiplicity events and so on. These unexpected observations have challenged our current understanding of physics phenomena in small systems. In particular, new questions have been raised such as whether these effects are driven by the QGP formation or other mechanisms mimicking the QGP behaviour.

To investigate more precisely the origin of signs of collectivity in small systems, the balance function can be used. The balance function reflects the chargedependent part of two-particle correlations. In nucleus-nucleus collisions, it allows probing different aspects of the QGP evolution such as the hadronization time, freeze-out conditions and the collective motion. The balance function width in $\Delta \eta$ and $\Delta \varphi$ was shown to be particularly sensitive to an increase in radial flow. This is reflected in the narrowing of the width in central with respect to peripheral events observed previously for charged particles in $\mathrm{Pb}-\mathrm{Pb}$ system, explained as a consequence of stronger radial flow in central collisions. Similar narrowing with increasing multiplicity was also observed for charged particles in $\mathrm{p}-\mathrm{Pb}$ and pp collisions, which was consistent with the idea of collective phenomena present in small systems. Such results motivated the following expectation for the measurement of the balance function for identified hadrons in small systems: if the multiplicity-dependent narrowing of the width is driven by the expansion of a QGP or other effects resembling "radial flow-like" patterns, one should measure more pronounced narrowing for heavier particles due to their higher sensitivity to the boost.

To verify this expectation, in this thesis the analysis of the balance function for identified hadrons in proton-proton collisions at $\sqrt{\mathrm{s}}=5.02 \mathrm{TeV}$ collected by the ALICE experiment is presented. The measurement for pion and kaon pairs is performed in the transverse momentum range of $0.2<\mathrm{p}_{\mathrm{T}}<2 \mathrm{GeV} / \mathrm{c}$, while for proton pairs the range of $0.5<\mathrm{p}_{\mathrm{T}}<2 \mathrm{GeV} / \mathrm{c}$ is used. All particle species are studied in the rapidity range of $|\mathrm{y}|<0.5$. Two-dimensional balance functions and its projections on $\Delta \eta$ and $\Delta \varphi$ are reported for all aforementioned particles, while results of the balance function width and yield are presented only for pions and protons. The latter is not shown for kaons due to the contribution from the $\phi$ resonance decaying into kaon pairs that affects the shape of the balance function distribution significantly. To investigate the exact effect of this decay, 
the simulation study using the PYTHIA8 Monte Carlo model was performed. It was found that after the removal of the $\phi$ resonance the near-side peak in the balance function distribution for all kaon pairs becomes much broader, therefore the final results for kaons should be reported with the $\phi$ meson being excluded in the data analysis. Furthermore, a similar study was done for pions since a large fraction of resonances decays into pion pairs. This helped to understand that the near-side depletion at $(\Delta y, \Delta \varphi)=(0,0)$ in the balance function for pion pairs is mainly caused by $\omega$ and $\rho^{0}$ mesons decays. In addition, the effect of the resonance removal on the multiplicity-dependent narrowing of the balance function width was studied for pion and kaon pairs. In both cases, it allowed to conclude that the exclusion of resonances only affects the absolute value of the width, but not the multiplicity dependence.

In the experimental results, while a clear narrowing of the balance function width with increasing multiplicity is observed for pion pairs, no significant multiplicity dependence for proton pairs is present, contrary to the expectation of observing more pronounced narrowing for heavier particles if "radial flow-like" effects are the main cause of the multiplicity-dependent narrowing. In addition, qualitatively similar result is seen in $\mathrm{p}-\mathrm{Pb}$ collisions. This suggests that some other effects that are not connected to collectivity might drive such behaviour in both systems. Furthermore, in the direct comparison between data points in $\mathrm{p}-\mathrm{Pb}$ and pp a clear scaling of the balance function width with multiplicity is observed which implies that the mechanism behind a multiplicity-dependent trend of the width is the same in small systems.

Results of the balance function width in the data are compared to corresponding predictions from the PYTHIA8 model with and without color reconnection. The color reconnection was proposed as one of alternative mechanisms at the microscopic level that can lead to "radial flow-like" patterns in pp collisions. This mechanism is based on the string fragmentation model, i.e. on the calculation of the probability to connect partons by color lines, or strings. PYTHIA8 without color reconnection predicts no significant multiplicity-dependent trend of the width for both particle species and, thus, fails to describe the data. PYTHIA8 with color reconnection predicts the narrowing of the width towards higher multiplicity in both dimensions for both pions and protons with more pronounced trend for protons. The comparison between experimental results and model predictions therefore suggests that data disfavour the color reconnection mechanism as implemented in PYTHIA8.

In order to extract stronger conclusions, these very interesting observations in $\mathrm{p}-\mathrm{Pb}$ and $\mathrm{pp}$ collisions should be compared to the corresponding results in $\mathrm{Pb}-\mathrm{Pb}$ collisions. Such comparison would allow to understand if the underlying physics 
phenomena of particle production are of a common origin across all collision systems. 


\section{Samenvatting}

Het hoofdzakelijke doel van het zware-ionen programma van het ALICE experiment op CERN is het bestuderen van de eigenschappen van het quark-gluonplasma (QGP), een ongebonden medium gemaakt van quarks en gluonen dat gecreëerd is in de botsingen tussen ultra-relativistische kernen. Zulke studies maken het mogelijk om het gedrag van sterk wisselwerkende materie bij hoge energie dichtheden en temperaturen te onderzoeken.

Een zeer korte levensduur van de QGP toestand die snel expandeert en afkoelt maakt het ontoegankelijk voor directe experimentele metingen, waardoor de eigenschappen bestudeerd moeten worden door de uiteindelijke deeltjes te meten die indirect bewijs kunnen leveren over het formeren van het QGP. Voorgaande studies van verschillende experimentele observabelen lieten zien dat het medium dat gecreëerd wordt in zware-ionen botsingen zich gedraagt als een bijna perfecte vloeistof, d.w.z. de expansie wordt correct beschreven door de vergelijkingen van relativistische viskeuze hydrodynamica gebruikmakend van zeer laag viskeuze waardes. Volgens deze omschrijving, de druk gradiënten leiden tot een collectieve beweging van alle geproduceerde deeltjes vanuit de reactiezone naar buiten met een collectief gemiddelde transverse snelheidsveld genaamd radiale flow. Tevens, de anisotropische flow effecten zijn ook aanwezig in niet-centrale botsingen, wanneer de ruimtelijke anisotropy van het overlap gebied tussen botsende kernen getransformeerd is naar de flow van de uiteindelijke toestand in de impulsruimte. Een van de meest prominente experimentele reflecties van de hydrodynamische expansie van het QGP is geobserveerd in twee-deeltjes hoek correlaties die bestudeerd zijn als functie van de relatieve pseudorapiditeit $\Delta \eta$ en azimutale hoek $\Delta \varphi$ van het deeltjes paar. De verhoging in de hoeveelheid deeltjes paren die zitten rond $\Delta \varphi \sim 0$ en uitbreiden over alle $\Delta \eta$ waardes, genaamd de nabij-zijde richel, is toegeschreven aan de lange-afstands correlaties ontstaan uit anisotropische flow.

Als een referentie voor de zware-ionen geval, d.w.z. om de effecten uit elkaar te halen die verbonden zijn met de formatie van een heet en hoge dichtheid QGP medium of koude nucleaire effecten, worden proton-kern of proton-proton 
gebruikt, waar het QGP niet verwacht wordt door de kleinere grote van deze systemen. Verrassend genoeg, recente metingen gedaan door meerdere experimenten bij RHIC en LHC onthullen ook de aanwezigheid van de nabij-zijde richel in hoge-multipliciteiten proton-kern en proton-proton botsingen. Deze ontdekking werd gevolgd door meerdere andere studies in kleine systemen waar soortgelijke effecten toegekend aan collectiviteit in zware-ionen botsingen waren gevonden, zoals de niet-nul resultaten van flow coëfficiënten berekend door niet alleen twee-, maar ook meerdere-deeltjes correlaties, de negatieve teken van vierdeeltjes cumulant, verharding van de deeltjes $\mathrm{p}_{\mathrm{T}}$ spectra in hoge- vergeleken met lage-multipliciteiten botsing en zo verder. Deze onverwachte observaties hebben onze huidige begrip van fysische fenomenen in kleine systemen uitgedaagd. In het bijzonder, nieuwe vragen zijn opgekomen zoals of deze effecten gedreven worden door de formatie van het QGP of door andere mechanismen die het gedrag van het QGP nabootsen.

Om meer precies de oorsprong van tekenen van collectiviteit in kleine systemen te onderzoeken, kan de balansfunctie gebruikt worden. De balansfunctie reflecteert de ladings-afhankelijke deel van twee-deeltjes correlaties. In kern-kern botsingen, het maakt het mogelijk om verschillende aspecten van de QGP evolutie zoals de hadronisatietijd, bevriezing condities en de collectieve beweging te meten. de breedte van de balansfunctie in $\Delta \eta$ en $\Delta \varphi$ laat een gevoeligheid zien voor een toename in radiale flow. Dit is gereflecteerd in de versmalling van de breedte in centrale in vergelijking met perifere botsingen, en is voorheen geobserveerd voor geladen deeltjes in het $\mathrm{Pb}-\mathrm{Pb}$ systeem, wat uitgelegd is als een consequentie van een sterkere radiale flow in centrale botsingen. Soortgelijke versmalling met toenemende multipliciteiten was ook geobserveerd voor geladen deeltjes in $\mathrm{p}-\mathrm{Pb}$ en $\mathrm{pp}$ botsingen, wat consistent was met het idee dat collectieve fenomenen present zijn in kleine systemen. Zulke resultaten motiveren de volgende verwachtingen voor de metingen van de balansfunctie voor geïdentificeerde hadronen in kleine systemen: als de multipliciteiten-afhankelijke versmalling van de breedte gedreven wordt door de expansie van het QGP of andere effecten lijkend op "radiale flowachtige" patronen, dan zou men meer opvallende versmalling voor zwaardere deeltjes moeten meten door hun hogere gevoeligheid voor de versnelling.

Om deze verwachting te verifiëren, wordt in deze thesis de analyse van de balansfunctie voor geïdentificeerde hadronen in proton-proton botsingen bij $\sqrt{\mathrm{s}}=$ $5.02 \mathrm{TeV}$ verzameld bij het ALICE experiment gepresenteerd. De meting voor pion en kaon paren is uitgevoerd in de transverse impuls bereik van $0.2<\mathrm{p}_{\mathrm{T}}<2$ $\mathrm{GeV} / \mathrm{c}$, terwijl voor proton paren een bereik van $0.5<\mathrm{p}_{\mathrm{T}}<2 \mathrm{GeV} / \mathrm{c}$ wordt gebruikt. Alle deeltjes soorten worden bestudeerd in het rapiditeit bereik van $|\mathrm{y}|<0.5$. Tweedimensionale balans functies en de projecties op $\Delta \eta$ en $\Delta \varphi$ 
zijn gerapporteerd voor alle voorgenoemde deeltjes, terwijl de resultaten van de balansfunctie breedte en opbrengst alleen gepresenteerd worden voor pionen en protonen. Het laatste wordt niet gepresenteerd voor kaonen door de contributie van de $\phi$ resonantie die vervalt in kaon paren wat de vorm van de balansfunctie distributie significant aantast. Om het exacte effect van dit verval te onderzoeken wordt een PYTHIA8 Monte Carlo model simulatiestudie uitgevoerd. Hier werd gevonden dat het verwijderen van de $\phi$ resonantie in de nabij-zijde richel de balansfunctie distributie voor alle kaon paren veel breder maakt, waardoor de uiteindelijke resultaten voor kaonen gerapporteerd zouden moeten worden met de $\phi$ meson uitgesloten in de data analyse. Tevens, een soortgelijke studie was gedaan voor pionen omdat een grote fractie van resonanties vervallen in pion paren. Dit heeft geholpen om te begrijpen dat de nabij-zijde uitdunning bij ( $\Delta y$, $\Delta \varphi)=(0,0)$ in de balansfunctie voor pion paren voornamelijk door het verval van $\omega$ en $\rho^{0}$ mesonen komt. In toevoeging, de effecten van de resonantie verwijdering op de multipliciteits-afhankelijke versmalling van de balansfunctie breedte was bestudeerd voor pion en kaon paren. In beide gevallen wordt er geconcludeerd dat de uitsluiting van resonanties alleen effect hebben op de absolute waarde van de breedte, maar niet op de multipliciteit afhankelijkheid.

In de experimentele resultaten, terwijl een heldere versmalling van de balansfunctie breedte met toenemende multipliciteit geobserveerd is voor pion paren, er geen significante multipliciteit afhankelijkheid is voor proton paren, tegengesteld tot de verwachting van het waarnemen van een meer uitgesproken versmalling voor zwaardere deeltjes als "radiale flow-achtige" effecten de hoofdzakelijke oorzaak zijn van de multipliciteits-afhankelijke versmalling. In toevoeging, kwalitatief vergelijkbare resultaten zijn geobserveerd in p-Pb botsingen. Dit suggereert dat andere effecten die niet verbonden zijn met collectiviteit dit gedrag kan veroorzaken in beide systemen. Bovendien, in de directe vergelijking tussen de datapunten in $\mathrm{p}-\mathrm{Pb}$ en pp wordt een heldere schaling van de balansfunctie breedte met de multipliciteit gezien wat impliceert dat het mechanisme achter de multipliciteits-afhankelijke trend van de breedte hetzelfde is in kleine systemen.

Resultaten van de balansfunctie breedte in de data zijn vergeleken met de corresponderende voorspellingen van het PYTHIA8 model met en zonder kleur herverbinding. De kleur herverbinding was voorgesteld als een van de alternatieve mechanismen op het microscopisch niveau dat kan leiden tot "radiale flow-achtige" patronen in pp botsingen. Dit mechanisme is gebaseerd op het snaar fragmentatie model, d.w.z. op de berekening van de kans om partonen te verbinden met kleur lijnen or snaren. PYTHIA8 zonder kleur herverbinding voorspelt geen significante multipliciteits-afhankelijke trend van de breedte voor beide deeltjes soorten en, dus, faalt om de data te beschrijven. PYTHIA8 
met kleur herverbinding voorspelt de versmalling van de breedte richting hogere multipliciteit in beide dimensies voor beide pionen en protonen met een meer uitgesproken trend voor protonen. Het vergelijk tussen experimentele resultaten en model voorspellingen daardoor suggereren dat de data geen voorkeur heeft voor het kleur herverbinding mechanisme zoals het geïmplementeerd is in PYTHIA8.

Om tot sterkere conclusies te komen, zouden deze zeer interessante observaties in $\mathrm{p}-\mathrm{Pb}$ en pp botsingen vergeleken moeten worden met de corresponderende resultaten in $\mathrm{Pb}-\mathrm{Pb}$ botsingen. Dit vergelijk zou het mogelijk maken om beter begrip te krijgen of the onderliggende fysische fenomenen van deeltjes productie een gezamenlijke oorsprong hebben in alle botsing systemen. 


\section{Bibliography}

[1] M. Breidenbach, J. I. Friedman, H. W. Kendall, E. D. Bloom, D. H. Coward, H. DeStaebler, J. Drees, L. W. Mo, and R. E. Taylor, "Observed Behavior of Highly Inelastic electron-Proton Scattering", Phys.Rev.Lett. 23 (1969) 935-939, DOI: 10.1103/PhysRevLett.23.935

[2] G. Hanson et al., "Hadron Production by $\mathrm{e}^{+} \mathrm{e}^{-}$Annihilation at Centerof-mass Energies Between 2.6 and $7.8 \mathrm{GeV}$. II. Jet Structure and Related Inclusive Distributions", Phys.Rev.D 26 (1982) 991, DOI: 10.1103/PhysRevD.26.991

[3] W. Bartel et al. (JADE Collaboration), "Observation of Planar Three Jet Events in $\mathrm{e}^{+} \mathrm{e}^{-}$Annihilation and Evidence for Gluon Bremsstrahlung", Phys.Lett.B 91 (1980) 142-147, DOI: 10.1016/0370-2693(80)90680-2

[4] J. C. Collins and M. J. Perry, "Superdense Matter: Neutrons Or Asymptotically Free Quarks?", Phys.Rev.Lett. 34 (1975) 1353, DOI: 10.1103/PhysRevLett.34.1353

[5] A. Chodos, R.L. Jaffe, K. Johnson, C.B.Thorne, "Baryon structure in the bag theory", Phys.Rev.D 10 (1974) 2599, DOI: 10.1103/PhysRevD.10.2599

[6] T. DeGrand, R.L. Jaffe, K. Johnson, J. Kiskis, "Masses and Other Parameters of the Light Hadrons", Phys.Rev.D 12 (1975) 2060, DOI: 10.1103/PhysRevD.12.2060

[7] J. Rafelski, "Melting Hadrons, Boiling Quarks - From Hagedorn Temperature to Ultra-Relativistic Heavy-Ion Collisions at CERN: With a Tribute to Rolf Hagedorn", Springer, Cham (2016), DOI: 10.1007/978-3-319-17545-4

[8] K. G. Wilson, "Confinement of quarks", Phys. Rev. D 10, 2445, DOI: 10.1103/PhysRevD.10.2445

[9] A. Bazavov et al. (HotQCD Collaboration), "The equation of state in (2+1)-flavor QCD", Phys.Rev.D 90 (2014) 094503, DOI: 10.1103/PhysRevD.90.094503, arXiv:1407.6387 [hep-lat] 
[10] F. Karsch, "Lattice QCD at High Temperature and the QGP", AIP Conf.Proc. 842 (2006) 1, 20-28, DOI: 10.1063/1.2220177, arXiv:heplat/0601013

[11] D. Boyanovsky, H.J. de Vega, D.J. Schwarz, "Phase transitions in the early and present Universe", Ann.Rev.Nucl.Part.Sci. 56 (2006) 441-500, DOI: 10.1146/annurev.nucl.56.080805.140539, arXiv:hep-ph/0602002

[12] M. G. Alford, K. Schwenzer, "What the Timing of Millisecond Pulsars Can Teach us about Their Interior", Phys.Rev.Lett. 113 (2014) 25, 251102, DOI: 10.1103/PhysRevLett.113.251102, arXiv:1310.3524 [astro-ph.HE]

[13] B.Alver, G.Roland, "Collision geometry fluctuations and triangular flow in heavy-ion collisions", Phys.Rev.C 81 (2010) 054905, Phys.Rev.C 82 (2010) 039903 (erratum), DOI: 10.1103/PhysRevC.82.039903, 10.1103/PhysRevC.81.054905, arXiv:1003.0194 [nucl-th]

[14] M.Ruggieri, S.Plumari, F.Scardina, V.Greco, "Quarks Production in the Quark-Gluon Plasma Created in Relativistic Heavy Ion Collisions", Nucl.Phys.A 941 (2015) 201-211, DOI: 10.1016/j.nuclphysa.2015.07.004, arXiv:1502.04596 [nucl-th]

[15] S. Schlichting, D. Teaney, "The First fm/c of Heavy-Ion Collisions", Ann.Rev.Nucl.Part.Sci. 69 (2019) 447-476, DOI: 10.1146/annurev-nucl101918-023825, arXiv:1908.02113 [nucl-th]

[16] H. Song, Y. Zhou, K. Gajdosova, "Collective flow and hydrodynamics in large and small systems at the LHC", Nucl.Sci.Tech. 28 (2017) 7, 99, DOI: 10.1007/s41365-017-0245-4, arXiv:1703.00670 [nucl-th]

[17] J. E. Bernhard, J. S. Moreland, S. A. Bass, "Bayesian estimation of the specific shear and bulk viscosity of quark-gluon plasma", Nat. Phys. 15, 1113-1117 (2019), DOI: 10.1038/s41567-019-0611-8

[18] M. Ploskon, "Heavy-ion collisions - hot QCD in a lab", arXiv:1808.01411 [hep-ex]

[19] M. L. Miller, K. Reygers, S. J. Sanders, P. Steinberg, "Glauber Modeling in High Energy Nuclear Collisions", Ann.Rev.Nucl.Part.Sci. 57 (2007) 205-243, DOI: 10.1146/annurev.nucl.57.090506.123020, arXiv:nucl-ex/0701025

[20] U. W Heinz, R. Snellings, "Collective flow and viscosity in relativistic heavy-ion collisions", Ann.Rev.Nucl.Part.Sci. 63 (2013) 123-151, DOI: 10.1146/annurev-nucl-102212-170540, arXiv:1301.2826 [nucl-th] 
[21] M. Luzum, H. Petersen, "Initial State Fluctuations and Final State Correlations in Relativistic Heavy-Ion Collisions", J.Phys.G 41 (2014) 063102, DOI: 10.1088/0954-3899/41/6/063102, arXiv:1312.5503 [nucl-th]

[22] B. Abelev et al. (ALICE Collaboration), "Centrality dependence of $\pi, \mathrm{K}$, p production in $\mathrm{Pb}-\mathrm{Pb}$ collisions at $\sqrt{\mathrm{s}}=2.6 \mathrm{TeV}$ ", Phys.Rev.C 88 (2013) 044910, DOI: 10.1103/PhysRevC.88.044910, arXiv:1303.0737 [hep-ex]

[23] B.I. Abelev et al (STAR Collaboration), "Systematic Measurements of Identified Particle Spectra in $\mathrm{pp}, \mathrm{d}+\mathrm{Au}$ and $\mathrm{Au}+\mathrm{Au}$ Collisions from STAR", Phys.Rev.C 79 (2009) 034909, DOI: 10.1103/PhysRevC.79.034909, arXiv:0808.2041 [nucl-ex]

[24] S.S. Adler et al (PHENIX Collaboration), "Identified Charged Particle Spectra and Yields in $\mathrm{Au}+\mathrm{Au}$ Collisions at $\sqrt{\mathrm{S}_{\mathrm{NN}}}=200 \mathrm{GeV}$ ", Phys.Rev.C 69 (2004) 034909, DOI: 10.1103/PhysRevC.69.034909, arXiv:nucl-ex/0307022

[25] A. Jaiswal, V. Koch, "A viscous blast-wave model for relativistic heavy-ion collisions", arXiv:1508.05878 [nucl-th]

[26] P.Kovtun, D.T.Son, A.O.Starinets, "Viscosity in Strongly Interacting Quantum Field Theories from Black Hole Physics", Phys.Rev.Lett. 94 (2005) 111601, DOI: 10.1103/PhysRevLett.94.111601, arXiv:hep-th/0405231

[27] U. Heinz, C. Shen, H. Song, "The viscosity of quark-gluon plasma at RHIC and the LHC", AIP Conf.Proc. 1441 (2012) 1, 766-770, DOI: 10.1063/1.3700674, arXiv:1108.5323 [nucl-th]

[28] S. Voloshin, Y. Zhang, "Flow Study in Relativistic Nuclear Collisions by Fourier Expansion of Azimuthal Particle Distributions", Z.Phys.C 70 (1996) 665-672, DOI: 10.1007/s002880050141, arXiv:hep-ph/9407282

[29] F. Noferini (for the ALICE Collaboration), "Anisotropic flow of identified particles in $\mathrm{Pb}-\mathrm{Pb}$ collisions at $\sqrt{\mathrm{S}_{\mathrm{NN}}}=2.76 \mathrm{TeV}$ measured with ALICE at the LHC", Nucl.Phys.A 904-905 (2013) 483c-486c, DOI: 10.1016/j.nuclphysa.2013.02.058, arXiv:1212.1292 [nucl-ex]

[30] J. Adam et al (ALICE Collaboration), "Higher harmonic flow coefficients of identified hadrons in $\mathrm{Pb}-\mathrm{Pb}$ collisions at $\sqrt{\mathrm{s}_{\mathrm{NN}}}=2.76 \mathrm{TeV}$ ", JHEP 09 (2016) 164, DOI: 10.1007/JHEP09(2016)164, arXiv:1606.06057 [nucl-ex]

[31] N. Borghini, P. M. Dinh, J. Ollitrault, "Flow analysis from multiparticle azimuthal correlations", Phys.Rev.C 64 (2001) 054901, DOI: 10.1103/PhysRevC.64.054901, arXiv:nucl-th/0105040 
[32] A. Bilandzic, R. Snellings, S. Voloshin, "Flow analysis with cumulants: direct calculations", Phys.Rev.C 83 (2011) 044913, DOI: 10.1103/PhysRevC.83.044913, arXiv:1010.0233 [nucl-ex]

[33] A. Bilandzic, C. H. Christensen, K. Gulbrandsen, A. Hansen, Y. Zhou, "Generic framework for anisotropic flow analyses with multi-particle azimuthal correlations", Phys.Rev.C 89 (2014) 6, 064904, DOI: 10.1103/PhysRevC.89.064904, arXiv:1312.3572 [nucl-ex]

[34] K. Aamodt et al (ALICE Collaboration), "Harmonic decomposition of twoparticle angular correlations in $\mathrm{Pb}-\mathrm{Pb}$ collisions at $\sqrt{\mathrm{S}_{\mathrm{NN}}}=2.76 \mathrm{TeV}$ ", Phys.Lett.B 708 (2012) 249-264, DOI: 10.1016/j.physletb.2012.01.060, arXiv:1109.2501 [nucl-ex]

[35] K. Aamodt et al (ALICE Collaboration), "Higher harmonic anisotropic flow measurements of charged particles in $\mathrm{Pb}-\mathrm{Pb}$ collisions at $\sqrt{\mathrm{S}_{\mathrm{NN}}}=2.76 \mathrm{TeV}$ ", Phys.Rev.Lett. 107 (2011) 032301, DOI: 10.1103/PhysRevLett.107.032301, arXiv:1105.3865 [nucl-ex]

[36] H.-J. Xu, Z. Li, H. Song, "High order flow harmonics of identified hadrons in 2.76 A TeV Pb+Pb collisions", Phys.Rev.C 93 (2016) 6, 064905, DOI: 10.1103/PhysRevC.93.064905, arXiv:1602.02029 [nucl-th]

[37] K. Aamodt et al (ALICE Collaboration), "Suppression of charged particle production at large transverse momentum in central $\mathrm{Pb}-\mathrm{Pb}$ collisions at $\sqrt{\mathrm{S}_{\mathrm{NN}}}=2.76 \mathrm{TeV} "$, Phys.Lett.B 696 (2011) 30-39, DOI: 10.1016/j.physletb.2010.12.020, arXiv:1012.1004 [nucl-ex]

[38] G. Bencedi (for the ALICE Collaboration), "Nuclear modification factor of charged particles and light-flavour hadrons in $\mathrm{p}-\mathrm{Pb}$ collisions measured by ALICE", arXiv:1609.05665 [nucl-ex]

[39] V. Khachatryan et al (CMS Collaboration), "Observation of Long-Range Near-Side Angular Correlations in Proton-Proton Collisions at the LHC", JHEP 09 (2010) 091, DOI: 10.1007/JHEP09(2010)091, arXiv:1009.4122 [hep-ex]

[40] S. Chatrchyan et al (CMS Collaboration), "Observation of long-range nearside angular correlations in proton-lead collisions at the LHC", Phys.Lett.B 718 (2013) 795-814, DOI: 10.1016/j.physletb.2012.11.025, arXiv:1210.5482 [nucl-ex]

[41] A. Ohlson, "Ridges in p-A (and pp) collisions", arXiv:1901.00747 [nucl-ex]

[42] A. Adare et al (PHENIX Collaboration), "Quadrupole Anisotropy in Dihadron Azimuthal Correlations in Central $\mathrm{d}+\mathrm{Au}$ Collisions at $\sqrt{\mathrm{S}_{\mathrm{NN}}}=$ 
200 GeV", Phys.Rev.Lett. 111 (2013) 21, 212301, DOI: 10.1103/PhysRevLett.111.212301, arXiv:1303.1794 [nucl-ex]

[43] G. Aad et al (ATLAS Collaboration), "Measurement with the ATLAS detector of multi-particle azimuthal correlations in $\mathrm{p}+\mathrm{Pb}$ collisions at $\sqrt{\mathrm{s}_{\mathrm{NN}}}=5.02 \mathrm{TeV} "$, Phys.Lett.B 725 (2013) 60-78, DOI: 10.1016/j.physletb.2013.06.057, arXiv:1303.2084 [hep-ex]

[44] S. Chatrchyan et al (CMS Collaboration), "Multiplicity and transversemomentum dependence of two- and four-particle correlations in $\mathrm{pPb}$ and $\mathrm{PbPb}$ collisions", Phys.Lett.B 724 (2013) 213-240, DOI: 10.1016/j.physletb.2013.06.028, arXiv:1305.0609 [nucl-ex]

[45] B. Abelev et al (ALICE Collaboration), "Multi-particle azimuthal correlations in $\mathrm{p}-\mathrm{Pb}$ and $\mathrm{Pb}-\mathrm{Pb}$ collisions at the CERN Large Hadron Collider", Phys.Rev.C 90 (2014) 5, 054901, DOI: 10.1103/PhysRevC.90.054901, arXiv:1406.2474 [nucl-ex]

[46] S. Acharya et al (ALICE Collaboration), "Investigations of Anisotropic Flow Using Multiparticle Azimuthal Correlations in pp, p- $\mathrm{Pb}$, Xe-Xe, and $\mathrm{Pb}-$ Pb Collisions at the LHC", Phys.Rev.Lett. 123 (2019) 14, 142301, DOI: 10.1103/PhysRevLett.123.142301, arXiv:1903.01790 [nucl-ex]

[47] B. Abelev et al (ALICE Collaboration), "Multiplicity Dependence of Pion, Kaon, Proton and Lambda Production in p-Pb Collisions at $\sqrt{\mathrm{S}_{\mathrm{NN}}}=5.02 \mathrm{TeV}$ ", Phys.Lett.B 728 (2014) 25-38, DOI: 10.1016/j.physletb.2013.11.020, arXiv:1307.6796 [nucl-ex]

[48] S. Acharya et al (ALICE Collaboration), "Multiplicity dependence of $\pi$, $\mathrm{K}$, and p production in pp collisions at $\sqrt{\mathrm{s}}=13 \mathrm{TeV}$ ", arXiv:2003.02394 [nucl-ex]

[49] S. Acharya et al (ALICE Collaboration), "Multiplicity dependence of lightflavor hadron production in pp collisions at $\sqrt{\mathrm{s}}=7 \mathrm{TeV}$ ", Phys.Rev.C 99 (2019) 2, 024906, DOI: 10.1103/PhysRevC.99.024906, arXiv:1807.11321 [nucl-ex]

[50] B. Abelev et al (ALICE Collaboration), "Centrality Dependence of $\pi, \mathrm{K}, \mathrm{p}$ Production in Pb-Pb Collisions at $\sqrt{\mathrm{S}_{\mathrm{NN}}}=2.76 \mathrm{TeV}$ ", Phys.Rev.C 88 (2013) 044910, DOI: 10.1103/PhysRevC.88.044910, arXiv:1303.0737 [hep-ex]

[51] P. Koch, B.Müller, J. Rafelski, "Strangeness in Relativistic Heavy Ion Collisions", Phys.Rept. 142 (1986) 167-262, DOI: 10.1016/0370-1573(86)90096-7 
[52] B. Abelev et al (ALICE Collaboration), "Multiplicity Dependence of Pion, Kaon, Proton and Lambda Production in p-Pb Collisions at $\sqrt{\mathrm{S} N N}=5.02 \mathrm{TeV} "$, Phys.Lett.B 728 (2014) 25-38, DOI: 10.1016/j.physletb.2013.11.020, arXiv:1307.6796 [nucl-ex]

[53] J. Adam et al (ALICE Collaboration), "Multi-strange baryon production in p-Pb collisions at $\sqrt{\mathrm{s}_{\mathrm{NN}}}=5.02 \mathrm{TeV}$ ", Phys.Lett.B 758 (2016) 389-401, DOI: 10.1016/j.physletb.2016.05.027, arXiv:1512.07227 [nucl-ex]

[54] J. Adam et al (ALICE Collaboration), "Enhanced production of multistrange hadrons in high-multiplicity proton-proton collisions", Nature Phys. 13 (2017) 535-539, DOI: 10.1038/nphys4111, arXiv:1606.07424 [nucl-ex]

[55] S. Acharya et al (ALICE Collaboration), "Anisotropic flow of identified particles in $\mathrm{Pb}-\mathrm{Pb}$ collisions at $\sqrt{\mathrm{S}_{\mathrm{NN}}}=5.02 \mathrm{TeV}$ ", JHEP 09 (2018) 006, DOI: 10.1007/JHEP09(2018)006, arXiv:1805.04390 [nucl-ex]

[56] V. Pacik (for the ALICE Collaboration), "Elliptic flow of identified hadrons in small collisional systems measured with ALICE", Nucl.Phys. A 982 (2019) 451-454, DOI: 10.1016/j.nuclphysa.2018.09.020, arXiv:1807.04538 [nucl-ex]

[57] V. Khachatryan et al (CMS Collaboration), "Long-range two-particle correlations of strange hadrons with charged particles in $\mathrm{pPb}$ and $\mathrm{PbPb}$ collisions at LHC energies", Phys.Lett.B 742 (2015) 200-224, DOI: 10.1016/j.physletb.2015.01.034, arXiv:1409.3392 [nucl-ex]

[58] V. Khachatryan et al (CMS Collaboration), "Evidence for collectivity in pp collisions at the LHC", Phys.Lett.B 765 (2017) 193-220, DOI: 10.1016/j.physletb.2016.12.009, arXiv:1606.06198 [nucl-ex]

[59] P. Bozek, W. Broniowski, G. Torrieri, "Mass hierarchy in identified particle distributions in proton-lead collisions", Phys.Rev.Lett. 111 (2013) 172303, DOI: 10.1103/PhysRevLett.111.172303, arXiv:1307.5060 [nucl-th]

[60] K. Werner, M. Bleicher, B. Guiot, Iu. Karpenko, T. Pierog, "Evidence for flow in $\mathrm{pPb}$ collisions at $5 \mathrm{TeV}$ from $\mathrm{v}_{2}$ mass splitting", Phys.Rev.Lett. 112 (2014) 23, 232301, DOI: 10.1103/PhysRevLett.112.232301, arXiv:1307.4379 [nucl-th]

[61] A. M. Sirunyan et al (CMS Collaboration), "Elliptic flow of charm and strange hadrons in high-multiplicity $\mathrm{pPb}$ collisions at $\sqrt{\mathrm{S}_{\mathrm{NN}}}=$ 8.16 TeV", Phys.Rev.Lett. 121 (2018) 8, 082301, DOI: 10.1103/PhysRevLett.121.082301, arXiv:1804.09767 [hep-ex] 
[62] S. Lim (for the ATLAS Collaboration), "ATLAS measurements of collective flow of heavy-flavor hadrons in small collision systems", https://cds.cern.ch/record/2709180

[63] C. Aidala et al (PHENIX Collaboration), "Creating small circular, elliptical, and triangular droplets of quark-gluon plasma", Nature Phys. 15 (2019) 3, 214-220, DOI: 10.1038/s41567-018-0360-0, arXiv:1805.02973 [nucl-ex]

[64] F. Gelis, E. Iancu, J. Jalilian-Marian, R. Venugopalan, "The Color Glass Condensate", Ann.Rev.Nucl.Part.Sci. 60 (2010) 463-489, DOI: 10.1146/annurev.nucl.010909.083629, arXiv:1002.0333 [hep-ph]

[65] A. Bzdak, B. Schenke, P. Tribedy, R. Venugopalan, "Initial state geometry and the role of hydrodynamics in proton-proton, proton-nucleus and deuteron-nucleus collisions", Phys.Rev.C 87 (2013) 6, 064906, DOI: 10.1103/PhysRevC.87.064906, arXiv:1304.3403 [nucl-th]

[66] M. Mace, V. V. Skokov, P. Tribedy, R. Venugopalan, "Hierarchy of Azimuthal Anisotropy Harmonics in Collisions of Small Systems from the Color Glass Condensate", Phys.Rev.Lett. 121 (2018) 5, 052301, DOI: 10.1103/PhysRevLett.121.052301, arXiv:1805.09342 [hep-ph]

[67] M. Mace, V. V. Skokov, P. Tribedy, R. Venugopalan, "Erratum: Hierarchy of Azimuthal Anisotropy Harmonics in Collisions of Small Systems from the Color Glass Condensate”, Phys.Rev.Lett. 123 (2019) 3, 039901 (erratum), DOI: 10.1103/PhysRevLett.123.039901 (erratum)

[68] W. Zhao, Y. Zhou, H. Xu, W. Deng, H. Song, "Hydrodynamic Collectivity in Proton-Proton Collisions at 13 TeV", Phys.Lett.B 780 (2018) 495-500, DOI: $10.1016 /$ j.physletb.2018.03.022, arXiv:1801.00271 [nucl-th]

[69] S. McDonald, C. Shen, F. Fillion-Gourdeau, S. Jeon, C. Gale, "A Detailed Study and Synthesis of Flow Observables in the IPGlasma+MUSIC+UrQMD Framework", Nucl.Phys.A 967 (2017) 393-396, DOI: 10.1016/j.nuclphysa.2017.05.053, arXiv:1704.05362 [nucl-th]

[70] C. Gale, S. Jeon, B. Schenke, P. Tribedy, R. Venugopalan, "Event-byevent anisotropic flow in heavy-ion collisions from combined Yang-Mills and viscous fluid dynamics", Phys.Rev.Lett. 110 (2013) 1, 012302, DOI: 10.1103/PhysRevLett.110.012302, arXiv:1209.6330 [nucl-th]

[71] B. B. Back et al. (PHOBOS Collaboration), "Centrality dependence of charged hadron transverse momentum spectra in $\mathrm{d}+\mathrm{Au}$ collisions at $\sqrt{\mathrm{S}_{\mathrm{NN}}}=200 \mathrm{GeV}$ ", Phys.Rev.Lett. 91 (2003) 072302, DOI: 10.1103/PhysRevLett.91.072302, arXiv:nucl-ex/0306025 
[72] B. Abelev et al (ALICE Collaboration), "Measurement of prompt D-meson production in $\mathrm{p}-\mathrm{Pb}$ collisions at $\sqrt{\mathrm{S}_{\mathrm{NN}}}=5.02 \mathrm{TeV}$ ", Phys.Rev.Lett. 113 (2014) 23, 232301, DOI: 10.1103/PhysRevLett.113.232301, arXiv:1405.3452 [nucl-ex]

[73] V. Khachatryan et al (CMS Collaboration), "Charged-particle nuclear modification factors in $\mathrm{PbPb}$ and $\mathrm{pPb}$ collisions at $\sqrt{\mathrm{s}_{\mathrm{NN}}}=5.02 \mathrm{TeV}$ ", JHEP 04 (2017) 039, DOI: 10.1007/JHEP04(2017)039, arXiv:1611.01664 [nucl-ex]

[74] J. Adam et al (ALICE Collaboration), "Centrality dependence of charged jet production in p-Pb collisions at $\sqrt{\mathrm{s}_{\mathrm{NN}}}=5.02 \mathrm{TeV}$ ", Eur.Phys.J.C 76 (2016) 5, 271, DOI: 10.1140/epjc/s10052-016-4107-8, arXiv:1603.03402v2 [nucl-ex]

[75] S. Trogolo (for the ALICE Collaboration), "D-meson production in $\mathrm{Pb}-\mathrm{Pb}$ collisions with ALICE at the LHC", arXiv:2001.05949 [nucl-ex]

[76] K. Dusling, R. Venugopalan, "Comparison of the Color Glass Condensate to di-hadron correlations in proton-proton and proton-nucleus collisions", Phys.Rev.D 87 (2013) 9, 094034, DOI: 10.1103/PhysRevD.87.094034, arXiv:1302.7018 [hep-ph]

[77] K. Dusling, P. Tribedy, R. Venugopalan, "Energy dependence of the ridge in high multiplicity proton-proton collisions", Phys.Rev.D 93 (2016) 1, 014034, DOI: 10.1103/PhysRevD.93.014034, arXiv:1509.04410 [hep-ph]

[78] Z.W. Lin, C.M. Ko, B.A. Li, B. Zhang, S. Pal, "A Multi-Phase Transport Model for Relativistic Heavy Ion Collisions", Phys.Rev.C 72 (2005) 064901, DOI: 10.1103/PhysRevC.72.064901, arXiv:nucl-th/0411110

[79] G.L. Ma, Z.W. Lin , "Predictions for $\sqrt{\mathrm{s}_{\mathrm{NN}}}=5.02 \mathrm{TeV} \mathrm{Pb}+\mathrm{Pb}$ Collisions from a Multi-Phase Transport Model", Phys.Rev.C 93 (2016) 5, 054911, DOI: 10.1103/PhysRevC.93.054911, arXiv:1601.08160v2 [nucl-th]

[80] G.L. Ma, A. Bzdak, "Long-range azimuthal correlations in proton-proton and proton-nucleus collisions from the incoherent scattering of partons", Phys.Lett.B 739 (2014) 209-213, DOI: 10.1016/j.physletb.2014.10.066, arXiv:1404.4129 [hep-ph]

[81] T. Sjostrand, S. Mrenna, P. Skands, "PYTHIA 6.4 Physics and Manual", JHEP 05 (2006) 026, DOI: 10.1088/1126-6708/2006/05/026, arXiv:hep$\mathrm{ph} / 0603175$

[82] C. Bierlich, G. Gustafson, L. Lölnnblad, A. Tarasov, "Effects of overlapping strings in pp collisions", JHEP 03 (2015) 148, DOI: 10.1007/JHEP03(2015)148, arXiv:1412.6259 [hep-ph] 
[83] A.O. Velasquez, P. Christiansen, E.C. Flores, I. M. Cervantes, G. Pai, "Color reconnection and flow-like patterns in pp collisions", Phys.Rev.Lett. 111 (2013) 4, 042001, DOI: 10.1103/PhysRevLett.111.042001, arXiv:1303.6326 [hep-ph]

[84] C. Bierlich, J.R. Christiansen, "Effects of Colour Reconnection on Hadron Flavour Observables", Phys.Rev.D 92 (2015) 9, 094010, DOI: 10.1103/PhysRevD.92.094010, arXiv:1507.02091 [hep-ph]

[85] S. Bass, P. Danielewicz, S. Pratt, "Clocking hadronization in relativistic heavy ion collisions with balance functions", Phys.Rev.Lett. 85 (2000) 26892692, DOI: 10.1103/PhysRevLett.85.2689, arXiv:nucl-th/0005044

[86] S. Pratt, "Identifying the Charge Carriers of the Quark-Gluon Plasma", Phys.Rev.Lett. 108 (2012) 212301, DOI: 10.1103/PhysRevLett.108.212301, arXiv:1203.4578 [nucl-th]

[87] S. Pratt, "General Charge Balance Functions, A Tool for Studying the Chemical Evolution of the Quark-Gluon Plasma", Phys.Rev.C 85 (2012) 014904, DOI: 10.1103/PhysRevC.85.014904, arXiv:1109.3647 [nucl-th]

[88] https://www.star.bnl.gov/central/physics/results/result.php?res=17\&cat=ebye

[89] T. Csorgo, "Review of HBT or Bose-Einstein correlations in high energy heavy ion collisions", J.Phys.Conf.Ser. 50 (2006) 259-270, DOI: 10.1088/1742-6596/50/1/031, arXiv:nucl-th/0505019

[90] V. Khachatryan et al (CMS Collaboration), "Multiplicity and rapidity dependence of strange hadron production in $\mathrm{pp}, \mathrm{pPb}$, and $\mathrm{PbPb}$ collisions at the LHC", Phys.Lett.B 768 (2017) 103-129, DOI: 10.1016/j.physletb.2017.01.075, arXiv:1605.06699 [nucl-ex]

[91] S. Acharya et al (ALICE Collaboration), "Multiplicity dependence of $\pi$, $\mathrm{K}$, and p production in pp collisions at $\sqrt{\mathrm{s}}=13 \mathrm{TeV}$ ", arXiv:2003.02394 [nucl-ex]

[92] J.D. Bjorken, "Highly relativistic nucleus-nucleus collisions: The central rapidity region", Phys.Rev.D 27 (1983) 140-151, DOI: 10.1103/PhysRevD.27.140

[93] H.U. Bengtsson, T. Sjostrand, "The Lund Monte Carlo for Hadronic Processes: Pythia Version 4.8", Comput.Phys.Commun. 46 (1987) 43, DOI: 10.1016/0010-4655(87)90036-1

[94] S. Pratt, C. Plumberg, "Determining the Diffusivity for Light Quarks from Experiment", arXiv:1904.11459 [nucl-th] 
[95] S. Pratt, C. Plumberg, "Evolving Charge Correlations in a Hybrid Model with both Hydrodynamics and Hadronic Boltzmann Descriptions", Phys.Rev.C 99 (2019) 4, 044916, DOI: 10.1103/PhysRevC.99.044916, arXiv:1812.05649 [nucl-th]

[96] P. Bozek, "The balance functions in azimuthal angle is a measure of the transverse flow", Phys.Lett.B 609 (2005) 247-251, DOI: 10.1016/j.physletb.2005.01.072, arXiv:nucl-th/0412076

[97] W. Broniowski, W. Florkowski, "Description of the RHIC $\mathrm{p}_{\mathrm{T}}$ spectra in a thermal model with expansion", Phys.Rev.Lett. 87 (2001) 272302, DOI: 10.1103/PhysRevLett.87.272302, arXiv:nucl-th/0106050

[98] J. Adams et al (STAR Collaboration), "Identified particle distributions in pp and $\mathrm{Au}+\mathrm{Au}$ collisions at $\sqrt{\mathrm{S}_{\mathrm{NN}}}=200 \mathrm{GeV}$ ", Phys.Rev.Lett. $92(2004)$ 112301, 10.1103/PhysRevLett.92.112301, arXiv:nucl-ex/0310004

[99] D. Drijard et al (CERN-College de France-Heidelberg-Karlsruhe Collaboration), "Density, charge and transverse momentum correlations of particles in non-diffractive proton-proton collisions at $\sqrt{\mathrm{s}}=52.5 \mathrm{GeV}$ ", Nucl.Phys.B 155 (1979) 269-294, DOI: 10.1016/0550-3213(79)90269-4

[100] D. Drijard et al (ACCDHW Collaboration), "Quantum number effects in events with a charged particle of large transverse momentum: (II). Charge correlations in jets", Nucl.Phys.B 166 (1980) 233-242, DOI: 10.1016/05503213(80)90226-6

[101] H. Aihara et al (TPC/Two Gamma Collaboration), "Observation of strangeness correlations in $\mathrm{e}^{+} \mathrm{e}^{-}$annihilation at $\sqrt{\mathrm{s}}=29 \mathrm{GeV}$ ", Phys.Rev.Lett. 53 (1984) 2199, Phys. Rev. Lett. 53 (1984) 2199-2202, DOI: 10.1103/PhysRevLett.53.2199

[102] H. Aihara et al (TPC/Two Gamma Collaboration), "Study of baryon correlations in $\mathrm{e}^{+} \mathrm{e}^{-}$annihilation at $\sqrt{\mathrm{s}}=29 \mathrm{GeV}$ ", Phys.Rev.Lett. 57 (1986) 3140, DOI: 10.1103/PhysRevLett.57.3140

[103] J. Adams et al (STAR Collaboration), "Narrowing of the Balance Function with Centrality in $\mathrm{Au}+\mathrm{Au}$ Collisions at $\sqrt{\mathrm{S}_{\mathrm{NN}}}=130 \mathrm{GeV}$ ", Phys.Rev.Lett. 90 (2003) 172301, DOI: 10.1103/PhysRevLett.90.172301, arXiv:nucl-ex/0301014

[104] X.N. Wang, M. Gyulassy, "HIJING 1.0: A Monte Carlo Program for Parton and Particle Production in High Energy Hadronic and Nuclear Collisions", Comput.Phys.Commun. 83 (1994) 307, DOI: 10.1016/00104655(94)90057-4, arXiv:nucl-th/9502021 
[105] R. Brun, F. Bruyant, F. Carminati, S. Giani, M. Maire, "GEANT Detector Description and Simulation Tool", DOI: 10.17181/CERN.MUHF.DMJ1

[106] B. Andersson, G. Gustafson, G. Ingelman, T. Sjostrand, "Parton fragmentation and string dynamics", Phys.Rept. 97 (1983) 31-145, DOI: 10.1016/0370-1573(83)90080-7

[107] C. Alt et al (NA49 Collaboration), "System size and centrality dependence of the balance function in $\mathrm{A}+\mathrm{A}$ collisions at $\sqrt{\mathrm{S}_{\mathrm{NN}}}=17.2 \mathrm{GeV}$ ", Phys.Rev.C 71 (2005) 034903, DOI: 10.1103/PhysRevC.71.034903, arXiv:hep-ex/0409031

[108] C. Alt et al (NA49 Collaboration), "Rapidity and energy dependence of the electric charge correlations in A+A collisions at the SPS energies", Phys.Rev.C 76 (2007) 024914, DOI: 10.1103/PhysRevC.76.024914, arXiv:0705.1122 [nucl-ex]

[109] M. Bleicher et al, "Relativistic Hadron-Hadron Collisions in the UltraRelativistic Quantum Molecular Dynamics Model (UrQMD)", J.Phys.G 25 (1999) 1859-1896, DOI: 10.1088/0954-3899/25/9/308, arXiv:hep$\mathrm{ph} / 9909407$

[110] B. Abelev et al (ALICE Collaboration), "Charge correlations using the balance function in $\mathrm{Pb}-\mathrm{Pb}$ collisions at $\sqrt{s_{N N}}=2.76 \mathrm{TeV}$ ", Phys.Lett.B 723 (2013) 267-279, DOI: 10.1016/j.physletb.2013.05.039, arXiv:1301.3756 [nucl-ex]

[111] J.Adam et al (ALICE Collaboration),"Multiplicity and transverse momentum evolution of charge-dependent correlations in pp, $\mathrm{p}-\mathrm{Pb}$, and $\mathrm{Pb}-\mathrm{Pb}$ collisions at the LHC", Eur.Phys.J.C 76 (2016) 2, 86, DOI: 10.1140/epjc/s10052016-3915-1, arXiv:1509.07255 [nucl-ex]

[112] A. Buckley et al, "General-purpose event generators for LHC physics", Phys.Rept. 504 (2011) 145-233, DOI: 10.1016/j.physrep.2011.03.005, arXiv:1101.2599 [hep-ph]

[113] G. Gustafson, "Multiple Interactions, Saturation, and Final States in pp Collisions and DIS", Acta Phys.Polon.B 40 (2009) 1981-1996, arXiv:0905.2492 [hep-ph]

[114] https://cds.cern.ch/record/2684277

[115] B. Abelev et al (ALICE Collaboration), "Performance of the ALICE Experiment at the CERN LHC", Int.J.Mod.Phys.A 29 (2014) 1430044, DOI: 10.1142/S0217751X14300440, arXiv:1402.4476 [nucl-ex] 
[116] K. Aamodt et al (ALICE Collaboration), "Alignment of the ALICE Inner Tracking System with cosmic-ray tracks", JINST 5 (2010) P03003, DOI: 10.1088/1748-0221/5/03/P03003, arXiv:1001.0502 [physics.ins-det]

[117] K. Aamodt et al (ALICE Collaboration), "The ALICE experiment at the CERN LHC", JINST 3 (2008) S08002, DOI: 10.1088/17480221/3/08/S08002

[118] B. Abelev et al (ALICE Collaboration), "Technical Design Report for the Upgrade of the ALICE Inner Tracking System", J.Phys.G 41 (2014) 087002, DOI: 10.1088/0954-3899/41/8/087002

[119] J. Alme et al, "The ALICE TPC, a large 3-dimensional tracking device with fast readout for ultra-high multiplicity events", Nucl.Instrum.Meth.A 622 (2010) 316-367, DOI: 10.1016/j.nima.2010.04.042, arXiv:1001.1950 [physics.ins-det]

[120] S.H. Morgan Jr, "Corrections to the Bethe-Bloch formula for average ionization energy loss of relativistic heavy nuclei Close collisions", Nuclear Instruments and Methods Volume 106, Issue 3, February 1973, Pages 429-435

[121] W. Blum, W. Riegler, L. Rolandi, "Particle Detection with Drift Chambers (2nd ed.)", Springer, Verlag (2008)

[122] G. Dellacasa et al (ALICE Collaboration), "Technical design report of the time projection chamber", https://cds.cern.ch/record/451098/files/open2000-183.pdf

[123] A. Alici et al (ALICE Collaboration), "The MRPC-based ALICE Time-OfFlight detector: status and performance", Nucl.Instrum.Meth.A 706 (2013) 29-32, DOI: 10.1016/j.nima.2012.05.004, arXiv:1203.5976 [physics.ins-det]

[124] P. Antonioli et al (ALICE Collaboration), "Upgrade of the ALICE readout and trigger system Technical Design report", https://cds.cern.ch/record/1603472

[125] E. Abbas et al (ALICE Collaboration), "Performance of the ALICE VZERO system", JINST 8 (2013) P10016, DOI: 10.1088/17480221/8/10/P10016, arXiv:1306.3130 [nucl-ex]

[126] B. Abelev et al (ALICE Collaboration),"Centrality determination of Pb$\mathrm{Pb}$ collisions at $\sqrt{\mathrm{s}_{\mathrm{NN}}}=2.76 \mathrm{TeV}$ with ALICE", Phys.Rev.C 88 (2013) 4, 044909, DOI: 10.1103/PhysRevC.88.044909, arXiv:1301.4361 [nucl-ex]

[127] E. Bruna, A.Dainese, M. Masera, F. Prino,"Vertex reconstruction for proton-proton collisions in ALICE", https://cds.cern.ch/record/1225497?ln=ru 
[128] P. Billoir, "Track Fitting With Multiple Scattering: A New Method", Nucl.Instrum.Meth.A 225 (1984) 352-366, DOI: 10.1016/01675087(84)90274-6

[129] T. Sjostrand, S. Mrenna, P.Z. Skands, "A Brief Introduction to PYTHIA 8.1", Comput.Phys.Commun. 178 (2008) 852-867, DOI: 10.1016/j.cpc.2008.01.036, arXiv:0710.3820 [hep-ph]

[130] B. B. Abelev et al. (ALICE Collaboration), "Freeze-out radii extracted from three-pion cumulants in $\mathrm{pp}, \mathrm{p}-\mathrm{Pb}$ and $\mathrm{Pb}-\mathrm{Pb}$ collisions at the LHC", Phys.Lett.B 739 (2014) 139-151, DOI: 10.1016/j.physletb.2014.10.034, arXiv:1404.1194 [nucl-ex]

[131] http://pdg.lbl.gov/2019/tables/rpp2019-sum-mesons.pdf 



\title{
Curriculum Vitae
}

\author{
2016 - 2020 \\ Utrecht University, \\ Nikhef
}

\section{PhD in Particle Physics}

Thesis: Studies of collective effects in pp collisions at the LHC with the balance function for identified particles

\footnotetext{
$2014-2016$

Warsaw University

of Technology
}

\footnotetext{
$2010-2014$

Warsaw University

of Technology
}

\author{
MSc in Nuclear Physics
}

Thesis: The hidden charm mesons production analysis in pp reactions at $\sqrt{\mathrm{s}}=500 \mathrm{GeV}$ in the STAR experiment

\section{BSc in Computer Physics}

Thesis: Nondestructive determination of thin layers thickness distribution by the measurement of $\alpha$ particles energy losses

Publication: http://link.springer.com/article/10.1140/epja/i201515015-2 



\section{Acknowledgements}

So, now I am writing this and... I guess it feels a bit like writing an Oscar speech.

Seriously, though, I can not believe it's been almost four years since I entered Nikhef for the first time and walked through its corridors to my new office. It still seems like this was yesterday and, at the same time, like there's still plenty of time ahead of me, but in reality, it's over. It's been truly an amazing journey in the world of real, beautiful science that I got a chance to become a part of. When I started studying physics at university, I couldn't even imagine doing my own academic research at some point and dealing with the data from the greatest lab we have ever built, CERN.

I am beyond grateful for all the experience this time has brought me, not only as a scientist, but also as a human being. I got to learn so much in terms of physics, but also in terms of life lessons - this has made me a completely different person than I used to be four years ago. Of course, arriving to the final destination of this journey depends a lot on how devoted and motivated you stay until the very end. But just as importantly, I think it wouldn't be possible without people who have helped me since my first day as a PhD student, so I would like to use this opportunity to say a big, big, big thank you to all of you.

Panos, thank you for always being ready to explain every little or big thing I needed to understand. Thank you for all your time and the will to answer endless amount of my questions (hmm, even strange questions sometimes...) and resolve those never-ending doubts. Thank you for being an example. Thank you for believing in me and always caring about me. You taught me being independent, confident and strong. I appreciate all the support you have given me very much and I told you more about what it means to me in person once, I hope you remember.

Raimond, thank you for enrolling me as a member of Dutch ALICE group, I would not be here now if not you. 
Paul, I knocked on your door quite often when I was stuck with something and nobody else was around, thank you for always being happy to help me in figuring things out. I am also thankful for the opportunity to work on the ITS upgrade for my service task in your team, which I found really cool.

Thomas, Marta, Alessandro, Marco, thank you for your valuable feedback and questions during the meetings or talk rehearsals.

Robert, I was lucky to have you as a member of my C3 committee, thank you for always being interested in my progress and curious about my research, thank you for all your advice and our long coffee talks that I enjoyed a lot.

I also want to thank the young part of our group for making this journey super fun :) Davide, Mike, Lennart, Goran, Dimitra, Luuk, Auro, Henrique, Christina, Barbara, Jacopo, Rihan, Steven, Sizar, Floris and others, it was great to meet all of you and share a lot of nice moments together.

Davide, you were the best postdoc I could have ever worked with. I respect and admire you and your work attitude so much, you know this. It was such a pleasure to share the office (I will never forget these bad and good, but mostly bad sudden expressions in Italian...) and the analysis with you all these years, almost everyday. Your help was just immeasurable, thank you for all our discussions, for the fact that I could always count on you in any situation and just being there for me whenever I needed it.

Auro, a separate thank you belongs to you for being the best teaching assistant buddy ever, I will always laugh reminding how confused we sometimes ended up after our tutorials, but we were always able to find a solution together and I've always had your back.

Mike, heel erg bedankt voor een geweldige vertaling van mijn samenvating! Ik hoop dat ik het ooit zelf kan doen...

Goran, thanks a lot for sharing your experience and knowledge (you know what I mean) all this time with me, for helping out with some tensor algebra and just for being Slavic :D

At the end, I want to say thank you for everything to my parents. I could probably write a separate book about what they've done and continue doing for me everyday, but I will save you now and may be do that later when I get my $\mathrm{PhD}$ degree haha.

I am so happy that I've done my $\mathrm{PhD}$ at Nikhef and in ALICE group. I will always smile coming back to these days in my memories. 